\title{
Risk and the midwife \\ A descriptive and interpretive examination of \\ the referral for obstetric consultation practices and attitudes \\ of New Zealand midwives
}

by

Joan Pius Skinner

A thesis submitted to the Victoria University of Wellington

in fulfillment of the

requirements for the degree of

Doctor of Philosophy

in Midwifery

Victoria University of Wellington 


\begin{abstract}
Risk is a dominant discourse in current formations of Western society. This thesis examines how risk is expressed in New Zealand midwifery by investigating both midwives' actions and midwives' attitudes. Risk is a complex concept and theoretical approaches to it come from a variety of perspectives. The techno rational approach stresses the quantifiable and probabilistic nature of risk; the social and cultural approaches are varied and stress the cultural embeddedness and value-laden nature of risk and its expression. Midwives must deal with risk from multiple and sometimes conflicting perspectives.
\end{abstract}

The philosophical approach of critical realism, which proposes that knowledge should be explored through multiple lenses and that knowledge is both fallible and emancipatory, provides the ontological and methodological support for the study. A national survey of midwives' practices and attitudes was undertaken in 2001, followed by six focus group discussions with midwives in a variety of settings.

The findings of the research reveal that midwives are both constrained by and act in resistance to risk. A model of midwifery is developed which illustrates the findings. The model is a three-legged birth stool, a birth stool for the midwife to sit on, rather than for the mother. The seat of the stool is called 'being with women'. This concept is central for New Zealand midwives, as they do not exclude women with risk factors from their care. They continue to provide care when risk is identified and when obstetricians need to be involved. The relationship they have with women is pivotal. The legs of the birth stool, which help give support to 'being with women', are: 'being a professional', 'working the system' and 'working with complexity'. The struts of the stool are 'storytelling', which help to keep the stool secure and stable. Midwives can use the stool as a tool to reflect on practice and to keep them connected to women. The stool can assist them in putting risk and its management into action and into perspective. Educators can use the stool to develop integrated and competent new midwives; managers can use it to provide systems that support the midwife; researchers can attend to areas of the birth stool that are less well understood. 


\section{Acknowledgements}

I wish to acknowledge Victoria University of Wellington for awarding me a $\mathrm{PhD}$ Scholarship to undertake this research. The scholarship allowed me the space and time to ponder, to think and to write. Thanks to the University's Faculty of Humanities and Social Sciences for the funding to undertake the fieldwork associated with this research. I was also awarded a Claude McCarthy Fellowship by the New Zealand Vice-Chancellors Committee to travel to the Netherlands, Denmark, the United Kingdom and Ireland.

Thanks must go to the staff of the Graduate School of Nursing and Midwifery, especially to Jan Duke, the Head of School. I was given both the space and the encouragement to undertake the project. Special thanks must go to Maralyn Foureur, my primary supervisor. She has the amazing knack of always giving the right feedback at the right time; no mean feat! Her support and encouragement were pivotal in keeping this project on track. She let me explore widely and then managed to help me get focused.

The participants in the research were all amazing. So many midwives took the time to fill out the questionnaire and I was very moved by their response. The midwives in the focus groups were generous in sharing their reflections with me and with each other. This process gave me great faith in midwives and in the thought and care that goes into their 'being with women'.

And finally thanks to my friends and especially to my family. You understood my distraction and my absences. You encouraged me and were proud of me and didn't ask too often when it was going to be finished. You had an unwavering faith that I was going to get it done. My husband Peter loved me and supported me through the whole process. I am so blessed to have him alongside.

This thesis is dedicated to Bronwen and Mele and all the women who have let me be with them and attend to them as they made their journey into motherhood. 


\section{Table of contents}

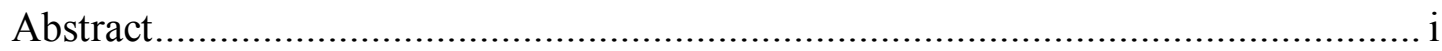

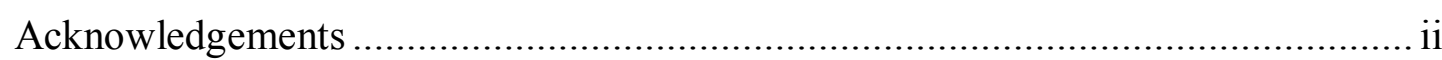

Table of contents ..................................................................................ii

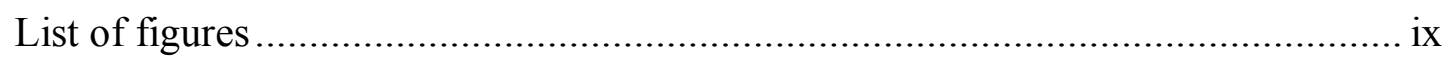

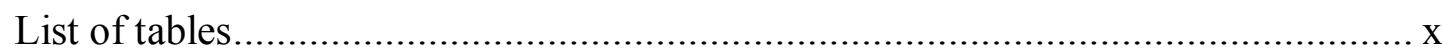

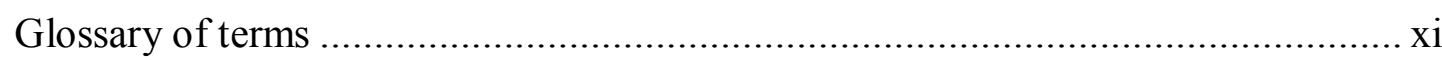

Chapter 1: Introduction ....................................................................1

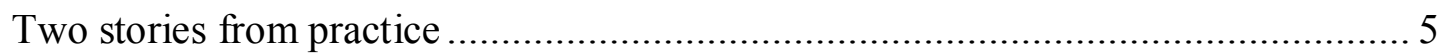

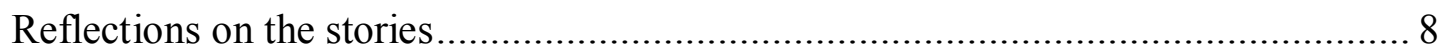

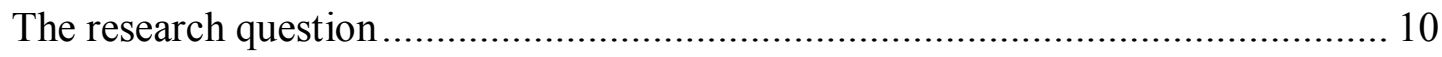

Midwifery and maternity care in New Zealand ................................................ 11

New Zealand and its childbearing population .............................................. 11

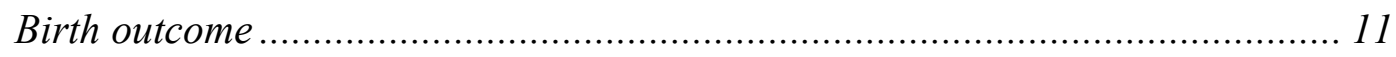

The recent history of New Zealand maternity care ........................................ 12

The current organisation of maternity care .................................................... 12

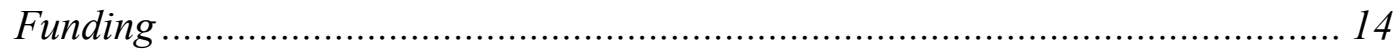

Professional organisation and education of midwives .................................. 15

The rights of the health consumer ............................................................ 15

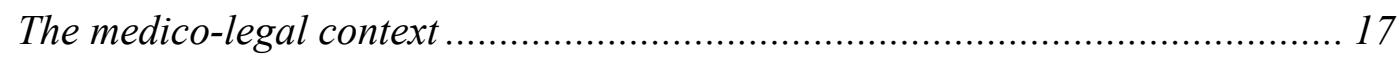

Referral for obstetric consultation - processes and problems ............................. 18

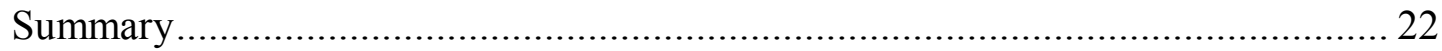

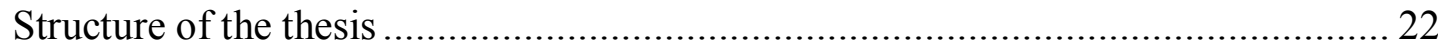

Chapter 2: Risk Theory .......................................................................25

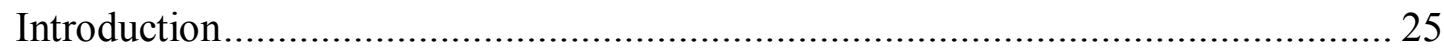

Techno-rational approaches to risk: chance and probability ................................. 26

The role of numbers and a place for normal................................................. 27

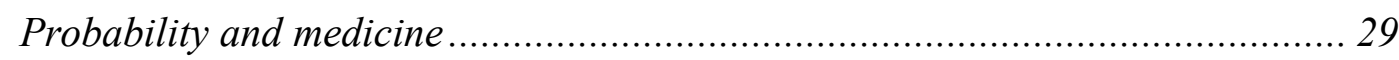

Risk and normality - a quandary for the midwife ........................................... 29

A problem with probability - the ecological fallacy ......................................... 31

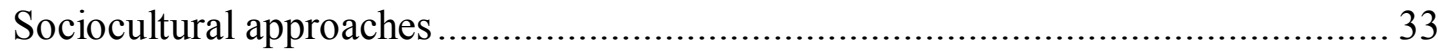

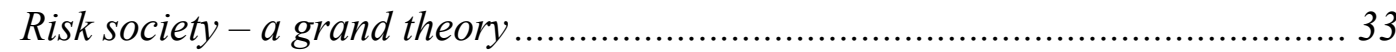




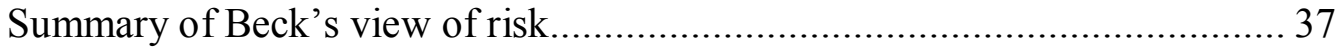

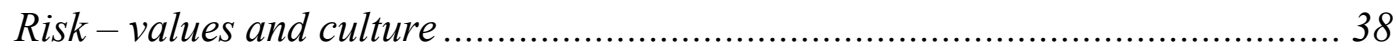

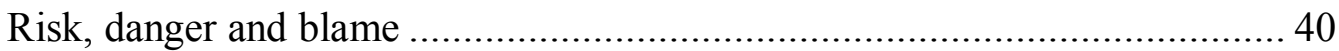

Summary of Douglas's view of risk........................................................ 43

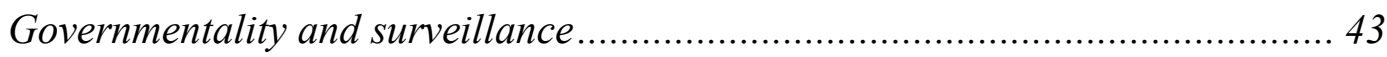

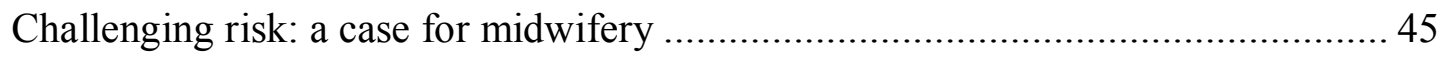

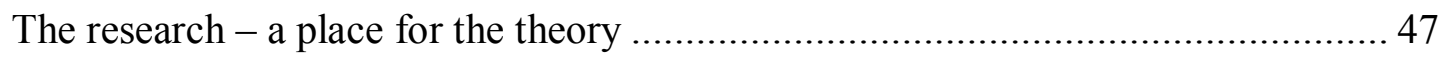

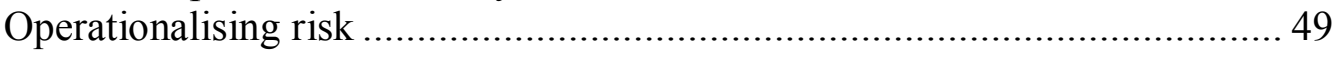

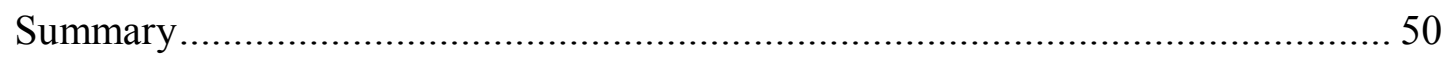

Chapter 3: Methodology .............................................................................52

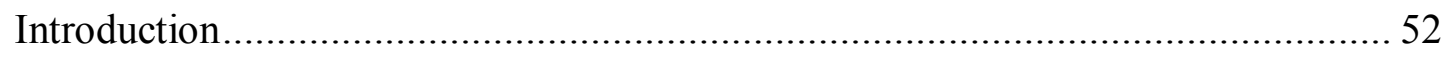

Where to stand: A place to be and a way to know ................................................ 53

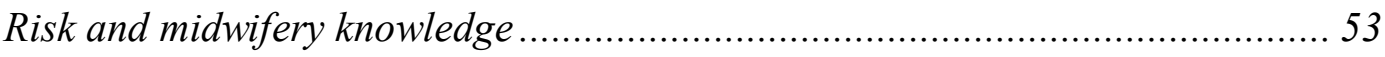

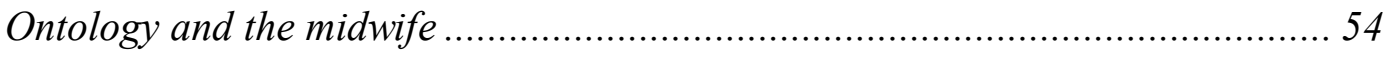

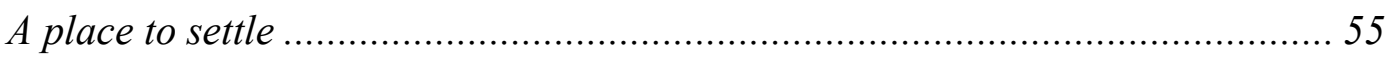

Critical realism: Help from a philosopher .......................................................... 56

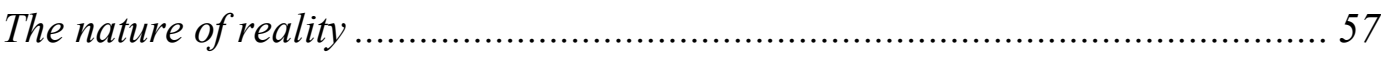

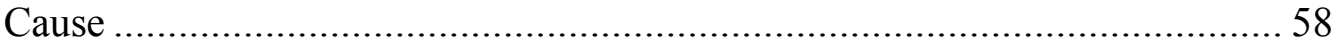

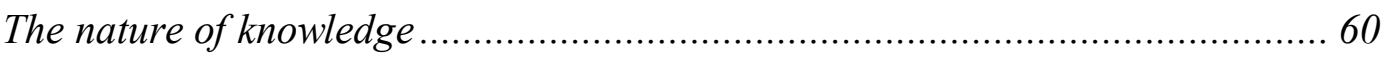

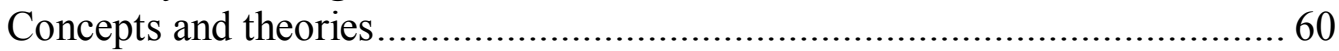

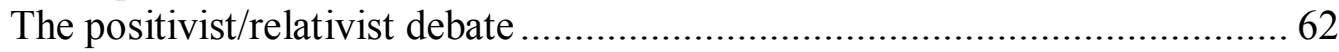

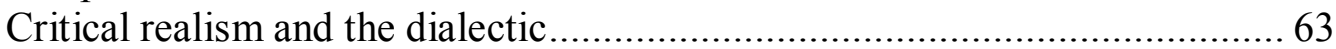

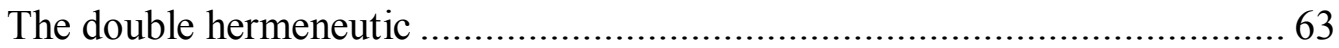

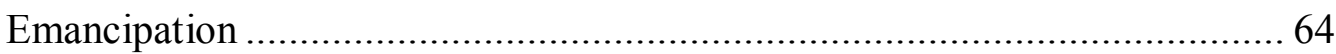

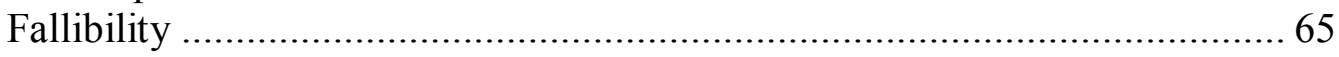

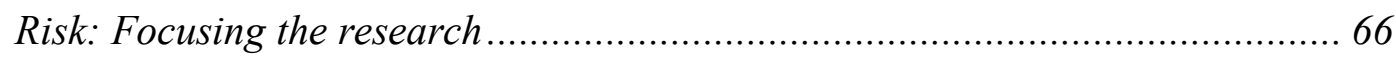

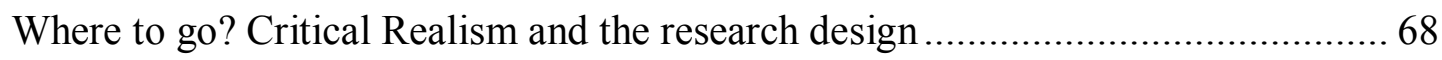

Intensive and extensive research methodologies ..................................... 71

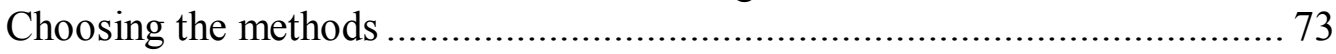

Putting the two together...................................................................... 77

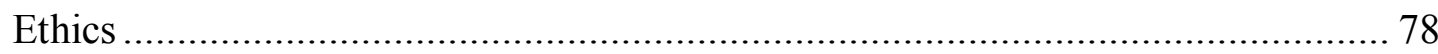

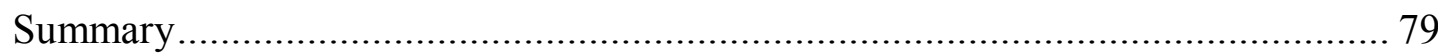

Chapter 4: The Survey - Design and Process..................................80

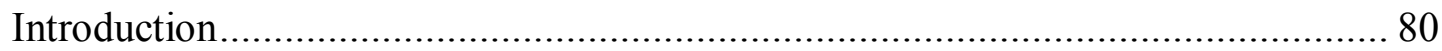

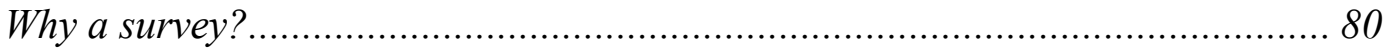

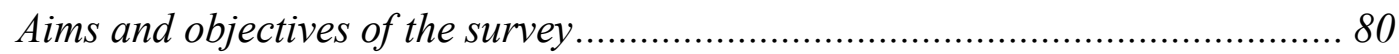

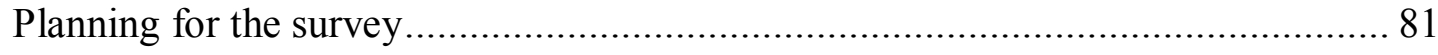

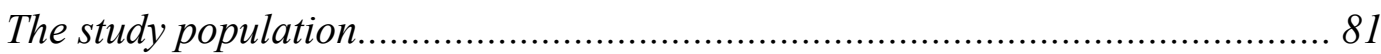




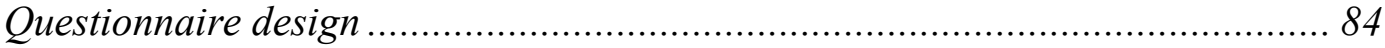

Demographic and work practice patterns of the midwives ......................... 85

Information about the referral processes and outcomes.............................. 85

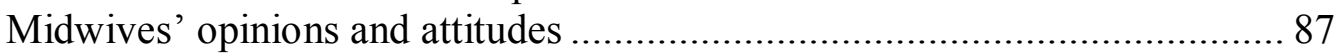

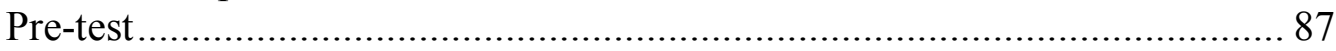

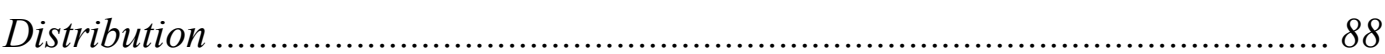

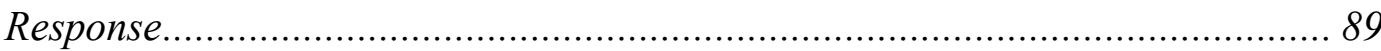

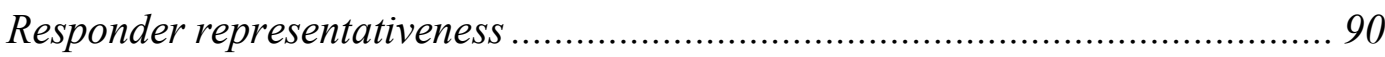

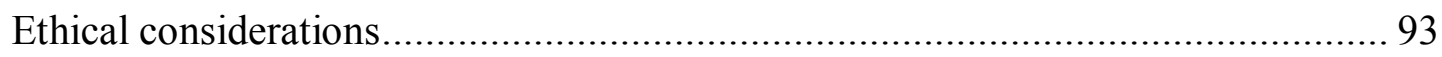

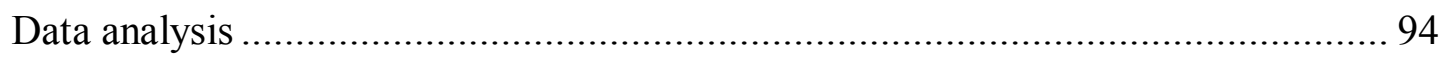

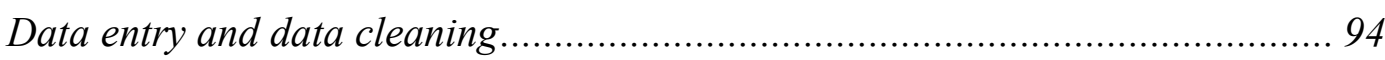

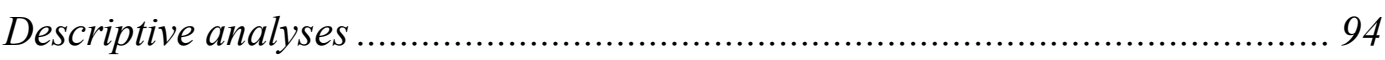

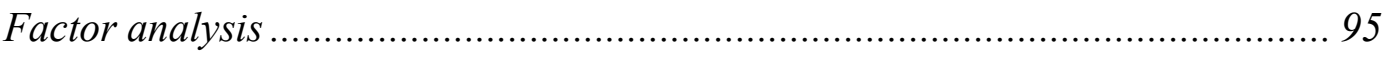

Relationships between the referral rate, the demographic characteristics, the practice patterns, and attitudes of the midwives ............................................. 96

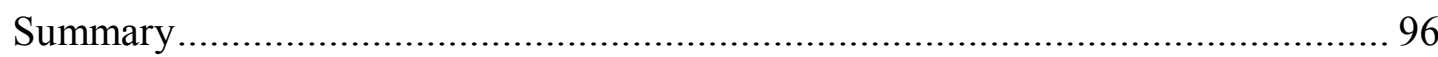

\section{Chapter 5: The Survey Results ........................................................97}

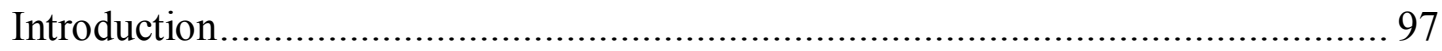

Section One: Description of the midwives, the referred women and the referral for

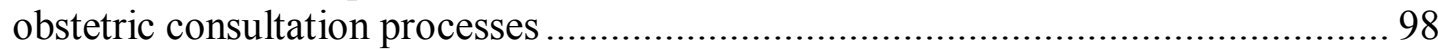

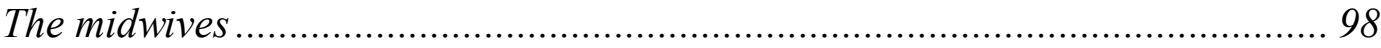

Demographic detail of the midwives ........................................................ 98

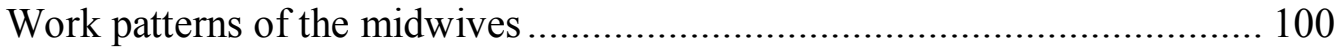

Location of the midwives ...................................................................... 100

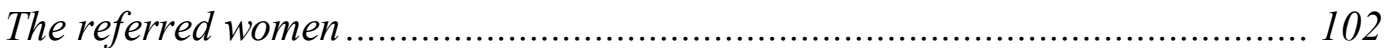

Demographic detail of the referred women........................................... 103

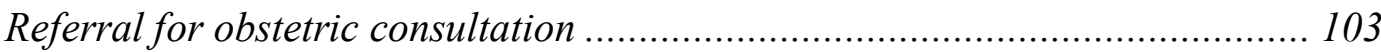

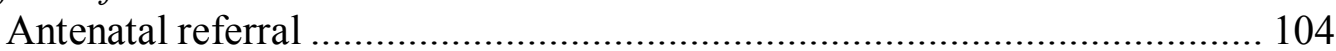

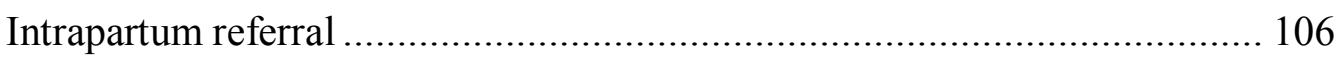

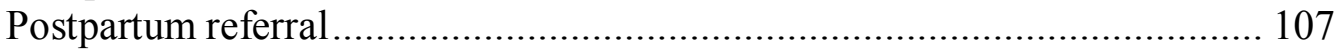

Transfer of clinical responsibility and LMC ........................................... 108

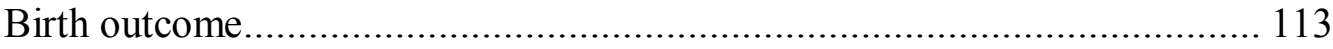

Section Two: Attitudes of the midwives ........................................................ 114

Responses to attitudinal statements ........................................................... 114

Section Three: Relationships between referral and the demographic and attitudinal characteristics of the midwives. ......................................................................... 118

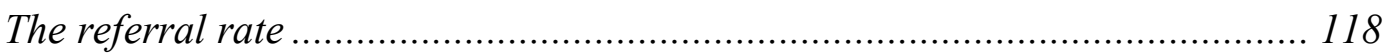

The caesarean section rate............................................................................ 120 
Attitudes, demographic characteristics and work patterns .......................... 120

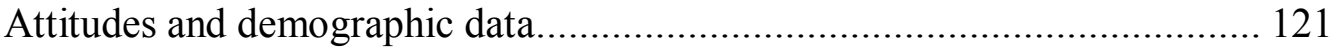

Relationships with the secondary maternity service ................................. 122

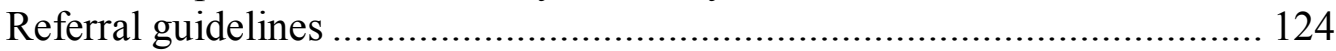

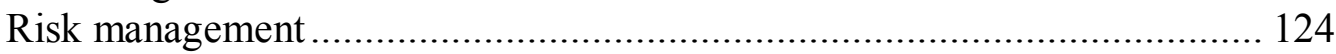

The care of high risk women ................................................................ 124

The medico-legal environment ...................................................... 125

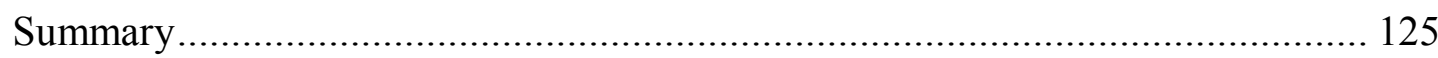

Chapter 6: Discussion of the Survey .....................................................126

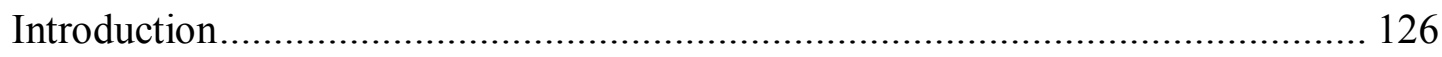

The LMC midwifery workforce in New Zealand ........................................ 127

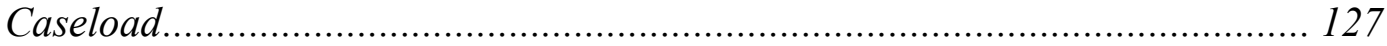

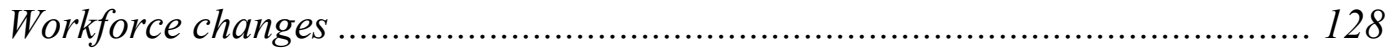

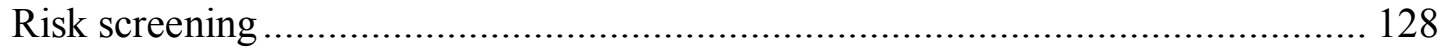

Referral and transfer of clinical responsibility................................................. 131

The referral rate .................................................................................. 131

Recommendations and guidelines for referral ....................................... 132

Comparisons of referral rates with other midwives and with GPs .............. 133

Commentary in the literature on referral rates........................................ 144

Referral patterns and midwifery characteristics ....................................... 145

Birth outcome and referral.................................................................... 148

Choosing the place of delivery .................................................................. 149

Consultation and continuing care ............................................................. 151

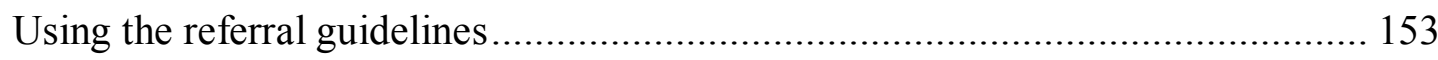

Referral guidelines in other countries ...................................................... 154

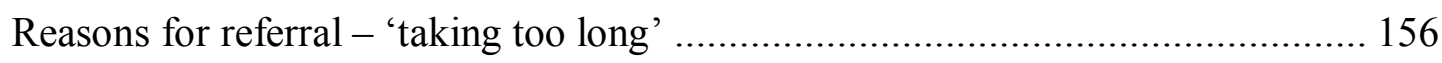

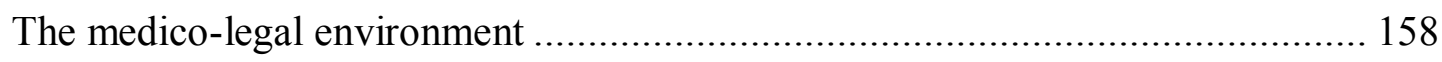

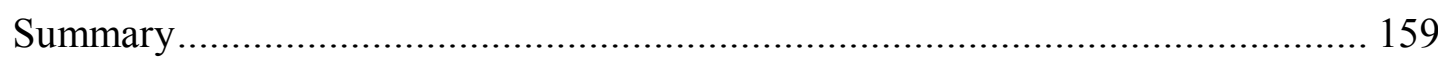

Chapter 7: Focus Groups - Design and Process .................................162

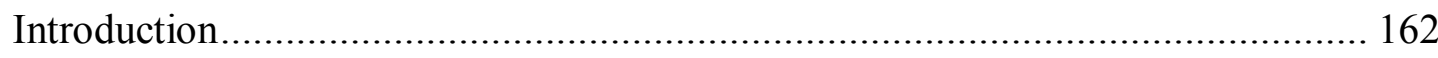

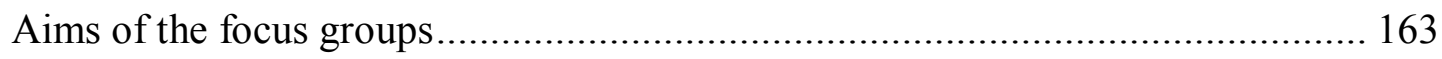

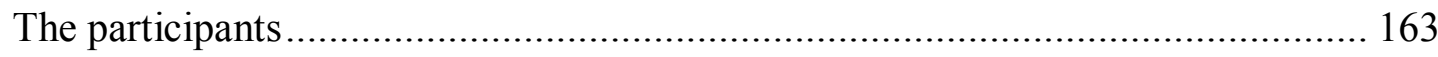

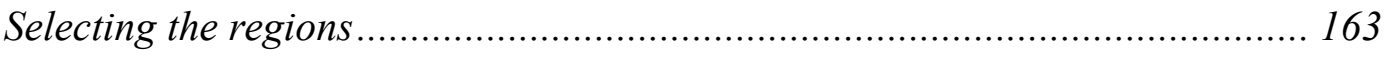

Recruiting the group members ............................................................. 164

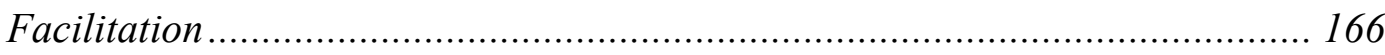

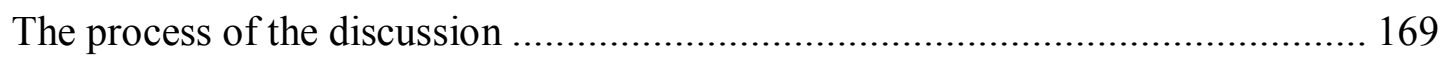

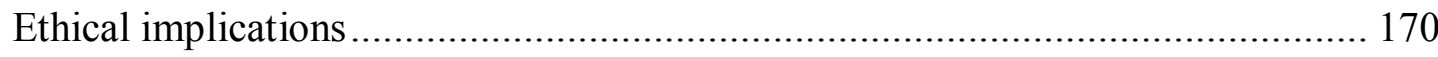




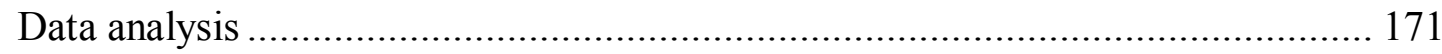

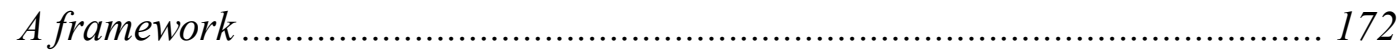

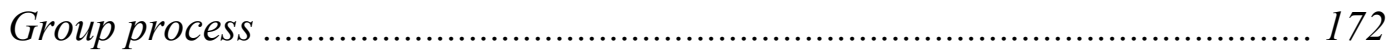

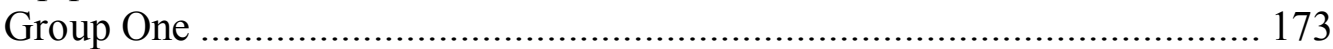

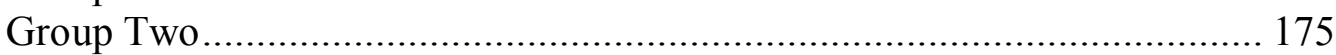

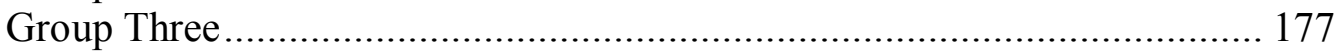

Group Four..................................................................................... 179

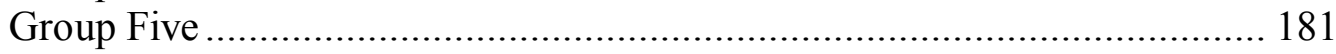

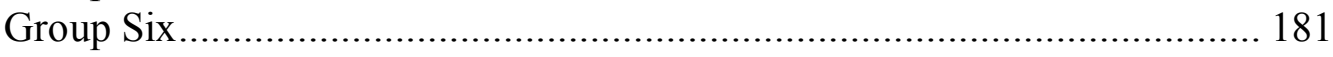

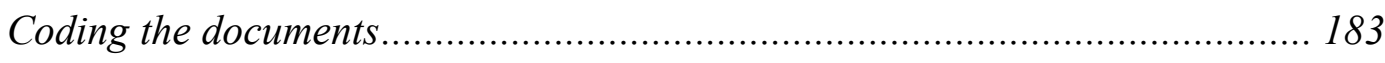

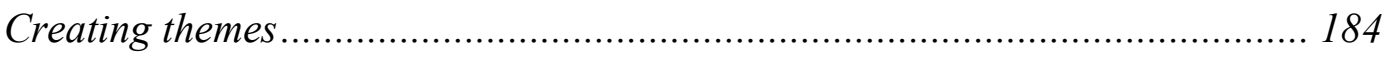

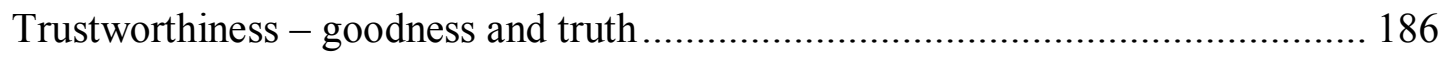

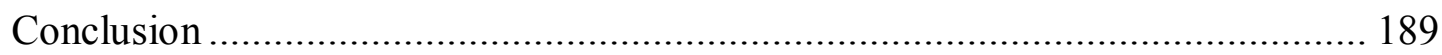

Chapter 8: Thematic Analysis and Interpretation.............................190

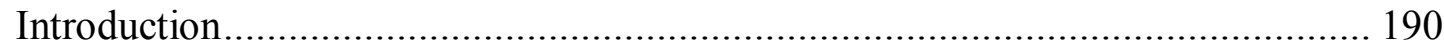

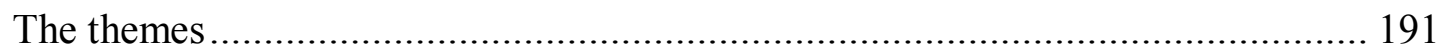

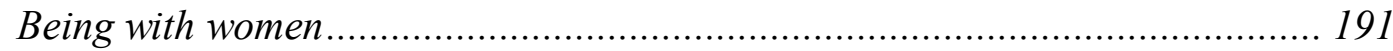

Holistic care - 'being their anchor' ....................................................... 192

Having a relationship - 'continuity and trust' ......................................... 193

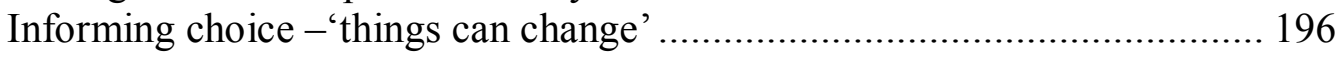

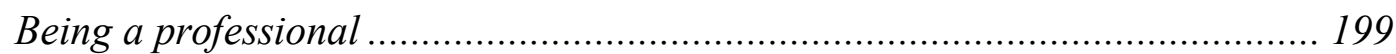

Being skilled - 'that's why you're a midwife' ...................................... 200

Being accountable - 'the eagle hovering'. ........................................... 207

Working the system ................................................................................... 212

Managing relationships - 'hoping like heck you get someone you like' ....... 213

Using the referral guidelines - 'whose permission do you want?' ................ 223

Power and authoritative knowledge - 'it's like the jungle' ......................... 226

Working with complexity....................................................................... 233

Being normal - 'excluding everybody that doesn't fit into the middle' ....... 233

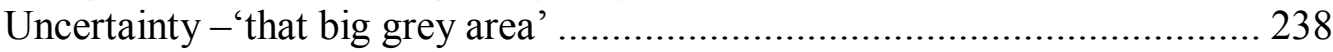

Multiple world views -'Between a rock and a hard place' ........................ 241

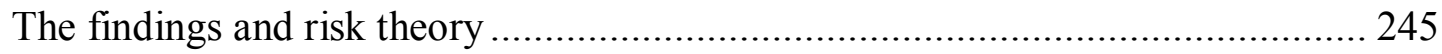

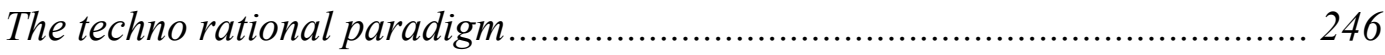

Beck's 'risk society' - lack of trust in the experts ......................................... 247

Douglas - blaming the 'Other'....................................................................... 251

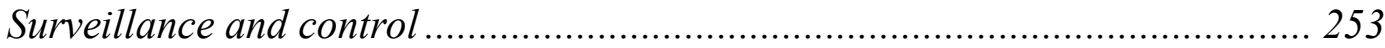

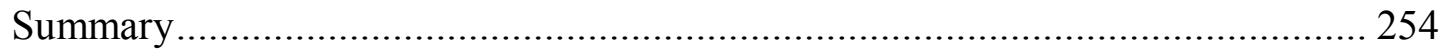


Chapter 9: A Birth Stool for the Midwife - a Model for Practice.256

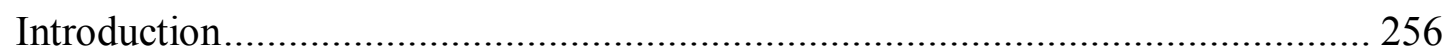

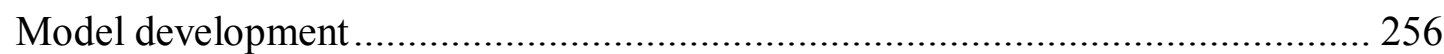

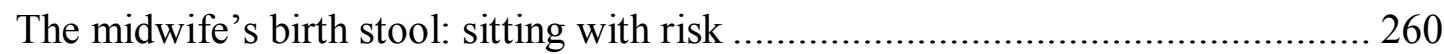

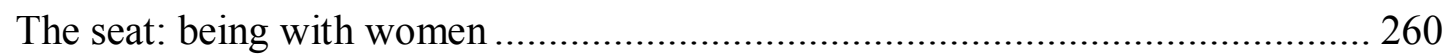

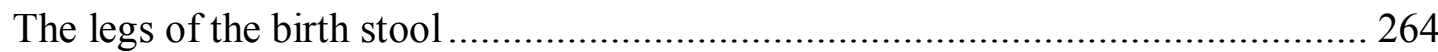

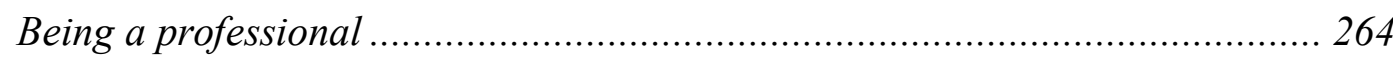

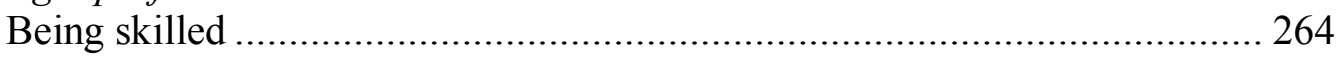

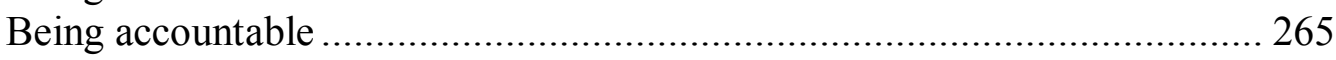

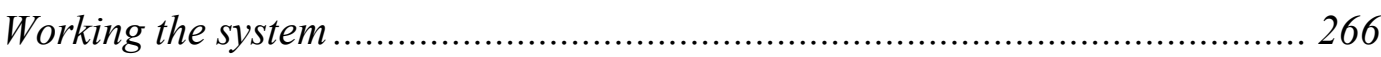

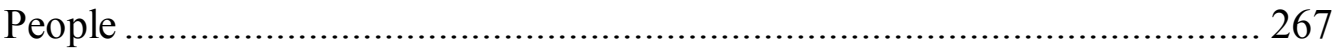

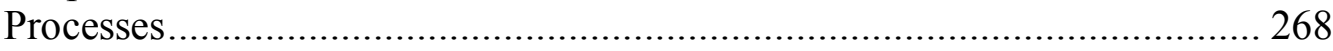

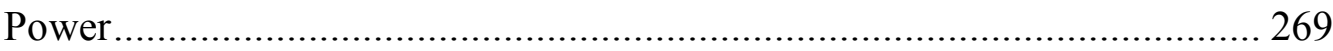

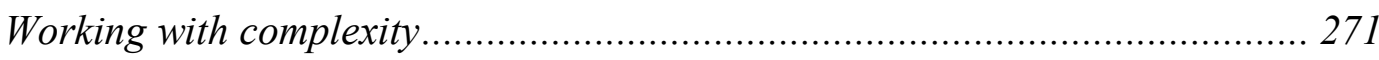

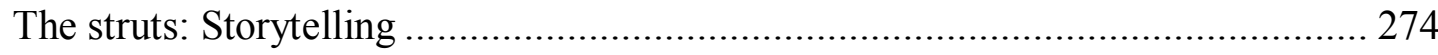

Sitting on the birth stool: Taking the model into practice …………...................... 276

The birth stool: What needs attention?............................................................. 278

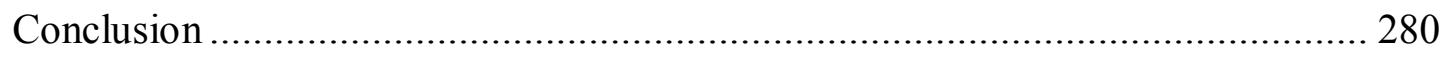

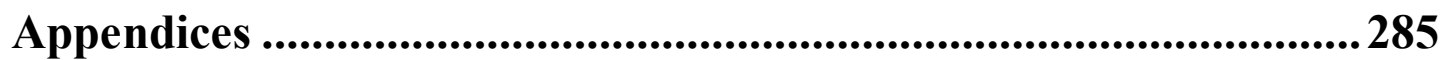

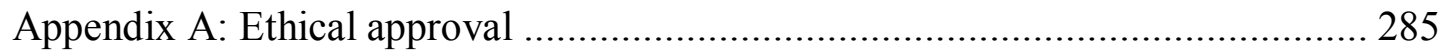

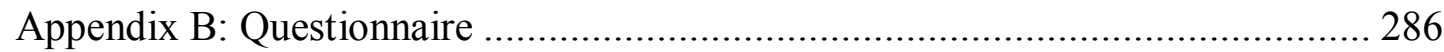

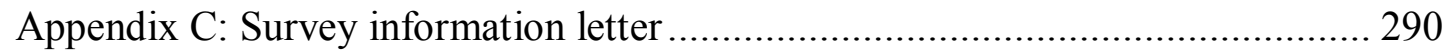

Appendix D: Focus group information letter and consent forms ............................ 293

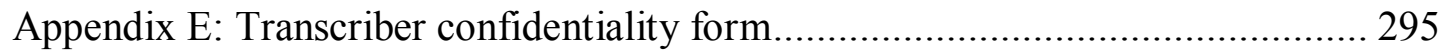

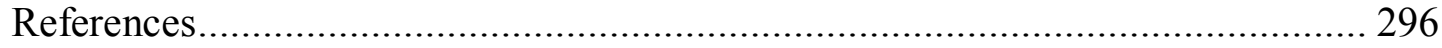




\section{List of figures}

Figure 1.1 The birth at home of Hana Olds......................................................... 5

Figure 1.2 The christening of Alakoka and Fangaifua in Tonga ............................ 7

Figure 4.1 Facility location of midwife respondents compared with the

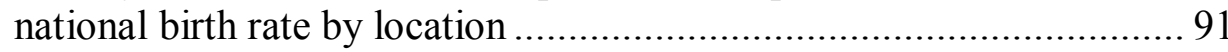

Figure 5.1 Distance from midwives' homes to secondary care hospital.............. 102

Figure 5.2 Reasons for antenatal referrals.................................................... 105

Figure 5.3 Reasons for intrapartum referrals............................................... 107

Figure 5.4 Reasons for postpartum referrals ................................................ 108

Figure 5.5 Referral and transfer of clinical responsibility ............................... 109

Figure 5.6 Continuation of midwifery care once clinical responsibility transferred ......................................................................... 110

Figure 5.7 Referral and transfer of clinical responsibility by childbearing

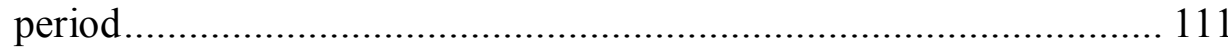

Figure 5.8 Independent midwives should care only for low risk women ............. 114

Figure 5.9 Risk management is an important part of my practice....................... 115

Figure 5.10 My pattern of referral is appropriate to the needs of the women I care for

Figure 5.11 The referral guidelines are a useful tool when I consider the need for referral.

Figure 5.12 The referral guidelines require me to refer more often than is necessary.....

Figure 5.13 In my region there is excellent collaboration between primary and secondary care

Figure 5.14 Obstetricians in my area in general support continuity of midwifery care

Figure 5.15 The medico-legal environment makes childbirth safer for women ..... 117

Figure 5.16 The medico-legal environment restricts me in my practice. .............. 117

Figure 5.17 A supportive and collaborative primary/secondary interface.............. 118

Figure 9.1 The midwife's birth stool: a model for midwifery practice................ 260 


\section{List of tables}

Table 1.1 Complaints made to Health and Disability Commission and breaches to the Code of Health and Disability Services

Consumers' Rights 18

Table 1.2 Examples of reasons and levels of referral taken from Guidelines for Consultation with Obstetric and Related Specialist Services (Ministry of Health, 2002) ................................................................. 19

Table 2.1 Theoretical perspectives on risk..................................................... 26

Table 3.1 Characteristics of intensive and extensive methodological

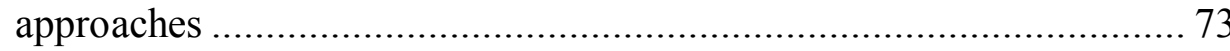

Table 4.1 Sources of LMC midwives for survey database ................................ 83

Table 4.2 Survey response ....................................................................... 90

Table 4.3 Comparison of midwifery demographic data with national data........... 91

Table 4.4 Comparison of details of referred women from study with other

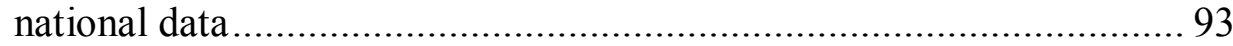

Table 5.1 Demographic and work pattern characteristics of midwives................ 99

Table 5.2 Location of referring hospital most frequently used by midwives, compared with national distributions of births by hospital. 101

Table 5.3 Demographic and birth outcome data of women referred for obstetric consultation. 103

Table 5.4 Numbers of women referred according to childbearing episode......... 104

Table 5.5 Practitioners to whom antenatal referrals were made ........................ 104

Table 5.6 Practitioners to whom intrapartum referrals were made ..................... 106

Table 5.7 Practitioners to whom postpartum referrals were made...................... 108

Table 5.8 Missing data where transfer of clinical responsibility is described

Table 5.9 Missing data where midwifery care continued after clinical responsibility handed over

Table 5.10 Differences in midwives attitudes according to main New Zealand Regions

Table 5.11 Degree to which midwives found the primary/secondary interface supportive according to hospital to which they referred....... 123

Table 6.1 Type of birth by original LMC in 2000 ........................................ 129

Table 6.2 Summary of referral rates found in national and international studies 134

Table 7.1 Characteristics of regions selected for focus group interviews ........... 165

Table 7.2 Characteristics of focus group participants ...................................... 166

Table 7.3 Focus group question guide ....................................................... 170

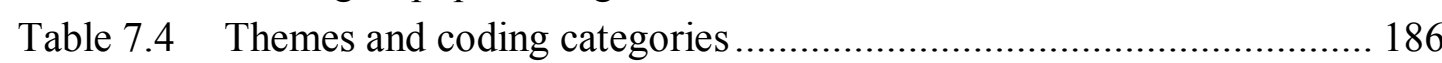




\section{Glossary of terms}

Access Agreements: A contract between the hospital and the LMC setting out the parameters by which the LMC can provide care within the facility.

Caseload midwives: midwives who provide a LMC service. Also referred to as 'independent' or 'self employed' midwives when they are not employed by a hospital.

Collaborative care: mutual consultation and joint planning between maternity providers in the provision of maternity care.

Core midwives: midwives who are employed in the hospital and work in shifts. They do not provide a LMC service but support LMCs to provide care. They provide midwifery care for complex need and also work in the postnatal wards.

Direct Entry midwives: Registered midwives who have not had a nursing education. Iwi-based Health Providers: Primary health services owned and controlled by Maori tribal groups. Sometimes employ midwives to provide a LMC service for their pregnant population.

LMC - Lead Maternity Caregiver: The health practitioner (midwife, GP or obstetrician) who the pregnant woman chooses to provide and coordinate all aspects of her care.

Midwifery Council of New Zealand: Took over the task of midwifery registration and regulation in 2004.

Nursing Council of New Zealand: The statutory, regulatory body, which until the end of 2004 was responsible for the registration of midwives and regulation of midwifery practice and education.

NZCOM: The New Zealand College of Midwives. The professional organisation of midwives. Responsible for the development and maintenance of professional standards support of its members.

Primary facility: a hospital or birth centre providing maternity care for uncomplicated childbirth.

Referral guidelines: Guidelines specifying the reasons and processes for referral for consultation with obstetric and related medical services.

Secondary facility: a hospital that provides a referral service, including core midwives, obstetricians, anaesthetists and paediatricians. Provides an epidural and caesarean section service and can deal with most complications of childbirth. 
Section 51 and Section 88: Government regulations setting out the terms and conditions for the purchase of primary maternity care and some referral maternity services from health providers. Contains service specifications, quality requirements, and process and payment rules. Section 88 relaced Section 51 in 2000.

Sign over of LMC: occurs when the childbearing woman has signed a document changing her nominated LMC. Excludes her previous LMC from participating in decision-making and from claiming payment.

Tertiary facility: A hospital that provides the full range of services including a neonatal intensive care unit.

Transfer of clinical responsibility: occurs when the clinical responsibility for decisions related to maternity care is transferred from the LMC to the obstetrician. The LMC can continue to participate in and make a claim for payment for care.

Union Health Centres: Community owned and controlled primary health services. Sometimes employ midwives to provide a LMC service for their pregnant population. 


\section{Chapter 1: Introduction}

Midwifery is about birth. Yet in its widest sense it is all about life itself. Birth, and therefore midwifery, is both real and sacred. It involves the whole person and the whole community and is a place where the spiritual and the soulful can be expressed. It is the work of the midwife to journey alongside the woman as she takes this key step along the life-death-life cycle. It is without doubt a profound moment in which two basic human experiences, love and fear, are both felt and expressed. The poet and cartoonist, Michael Leunig has written about these experiences that, he proposes, lie at the core of human existence:

There are only two feelings. Love and fear.

There are only two languages. Love and fear.

There are only two activities. Love and fear.

There are only two motives, two procedures, two frameworks, two results. Love and fear.

Love and Fear (Leunig, 1990).

At its most fundamental level this thesis addresses how love and fear are expressed within midwifery practice. Midwives are immersed in an experience that has the potential for both great love and great fear. We have the possibility of being connected with women and their families that few others could even dream about. How we feel and how we act has an impact on their birth experience. Skilful, loving action can make possible a truly wonderful birth; careless or fearful action can cause great damage. However birth and midwifery are also embedded in the context of social and cultural norms. This context is integral to how love and fear are expressed so are included in the perspective of this thesis.

Risk is the central concept examined in this thesis, as risk, and the way it is expressed relate closely to the notion of anxiety and fear. Risk also opens up the possibility of concerned and loving action. It poses the possibility of suffering harm or loss, yet also makes possible truly great human achievement. There is some contention about the origin of the word. It may have come from the Arabic risq which means good fortune, the Greek rhiza, meaning cliff, or the Latin word resegare, to cut off short 
(Wilkinson, 2001). Each has it own implications. Current constructions of risk however usually interpret it as being a negative concept to be avoided at all cost (Bernstein, 1996). Humans are seen as either being able to overcome it due to our ability to identify and measure it or as being vulnerable to it due to its unpredictability (Wilkinson, 2001). There appears little attention given to the positive aspects of risk taking; the good fortune that might occur should we 'take a risk'. It would seem that we are following the Greek and Latin meanings rather than the Arabic!

Risk is an issue of central concern in Western societies and has a strongly negative connotation. It would seem that the world has become a much more dangerous and therefore riskier place. We have a focus on managing and avoiding risk in all areas of our lives, personal, societal and global. From finance, business, and the environment, to education, welfare, and health, the language of risk has become central. We assess, predict, communicate, avoid and manage risk. Quality assurance managers have been renamed risk managers. Whole enterprises have been developed just to deal with risk and there is an enormous body of literature that develops and discusses multiple models of risk assessment and management. Yet this focus on risk has occurred during a time when we, at least in the West, have never been safer. Our life expectancy has increased and morbidity rates for many illnesses have decreased.

Midwifery is not immune from this process. Risk has also therefore become a defining concept for the midwife. One might propose that the midwife exists because of risk. We assess risk, manage risk, and communicate risk. This is really nothing new. To some extent this has always been our work. From the earliest times we have worked alongside women as they gave birth and were expected to be able to provide assistance if difficulties occurred (Rosenburg \& Trevathan, 2002). However there seems to be something about how risk is currently constructed, in the West at least, that has changed the way we do this. In the 1960s we first saw the beginning of women with complex health needs in pregnancy being defined as 'at risk', and the transfer of birth from the home to the hospital was based on the idea that all birth was risky (Tew, 1995). Since then the notion of 'at risk' pregnancies has been adopted worldwide. Complex scoring systems were developed in an attempt to differentiate low-risk from high-risk pregnancies (Enkin et al., 2000). Protocols and guidelines have been written in order to manage those deemed to be 'at risk' and some maternity 
units are physically divided into low and high-risk areas. Increasingly every pregnant woman is being defined as being 'at risk' of something and women are now more likely to give birth in secondary and tertiary hospitals rather than in primary birth facilities or at home. What used to be thought a normal part of life is increasingly considered fraught with danger. In some sense the dominance of this risk paradigm has caused a profound shift in the way we perceive birth. For the midwife the hope of providing care that enhances and protects what is a normal part of life has been increasingly constrained by societal attitudes dominated by risk and its control. The dominance of this risk paradigm is reflected in increasing rates of birth intervention worldwide and there is currently a significant movement attempting to understand why this is happening and to reverse this trend (Downe, 2004; Richard, 2001; Saxell, 2000; Walsh, El-Nemer, \& Downe, 2004; World Health Organisation, 1998, 2004).

I also have chosen risk as the central concept of concern because I have found it to be increasingly prominent in my own practice and in the practice of my peers. The issue in this research therefore, also comes from my own experience and is related to fear around birth. Risk itself has become fearful. The natural anxiety associated with birth, which motivates mothers to seek assistance and motivates midwives to be skilful and attentive seems to have become out of kilter. There is something about the current environment that has caused this anxiety to escalate into fear. I myself am much more anxious about birth complications than I used to be, even though I now have much greater skill and knowledge to assess and manage these. On the way to assist at a birth I now have a growing feeling of dread and have spent much time reflecting on this. Have I been at this birth business too long? Am I just getting burnt out? What am I afraid of? How does it affect my practice? What is the risk and do I have it in perspective? Do other midwives feel this? If they do, what affect is it having? Does it have something to do with the pressures of intervention or maybe the fear of litigation? I have found for me that it has, in some sense, 'spoiled' practice and I wondered if other midwives felt the same. This research then, is motivated by wondering about why there is so much fear about risk, what impact it has and how midwives manage to make sense of risk in their practice. It is essentially asking about the place of love and fear in the working world of the midwife. 
My perspective on this research is both personal and professional. I am a midwife with many years of experience in a wide variety of settings. In my 27 years of practice I have cared for all sorts of women in all sorts of settings; in tertiary hospitals, secondary hospitals, in delivery units, antenatal and postnatal wards and homes. I have been an educator and manager. I have been both employed in hospitals and health services, and been self-employed. I have been very involved in the professional midwifery organisation and been a midwifery activist. I have reviewed midwifery practice in many settings and have provided expert advice to Coroners, Health Boards and professional disciplinary bodies. I now teach midwifery at postgraduate level, supporting practising midwives to expand their knowledge and deepen their wisdom. In my life as a midwife I have seen many changes in the way maternity care has been provided and changes too in my own attitudes to birth and to the role of women. I have listened to many birth stories and told quite a few myself.

This research takes a critical realist approach, as outlined in chapter 3. . This approach supports investigation through multiple lenses. One of the lenses that I have chosen to is my own. Therefore I begin this research by providing my own perspectives and experiences in order for the reader to understand appreciate this lens. Critical realism proposes that although there is such a thing as 'reality' it is always viewed through a lens and that the truth that is found is always fallible. There is no such thing as objectivity. My own lens therefore is integral in this research. This first chapter begins this process by providing two stories of midwifery as it is currently experienced in New Zealand. These stories embed the thesis in the real and complex work of midwifery. The stories tell of both love and fear. They reveal how risk is played out in practice and are examples of the referral for obstetric consultation as a key process in midwifery's management of risk. The stories are followed by an initial reflection in which many of the issues are raised. The research question is then made explicit. The remainder of this chapter provides the context of the research and introduces the structure of this thesis. 


\section{Two stories from practice}

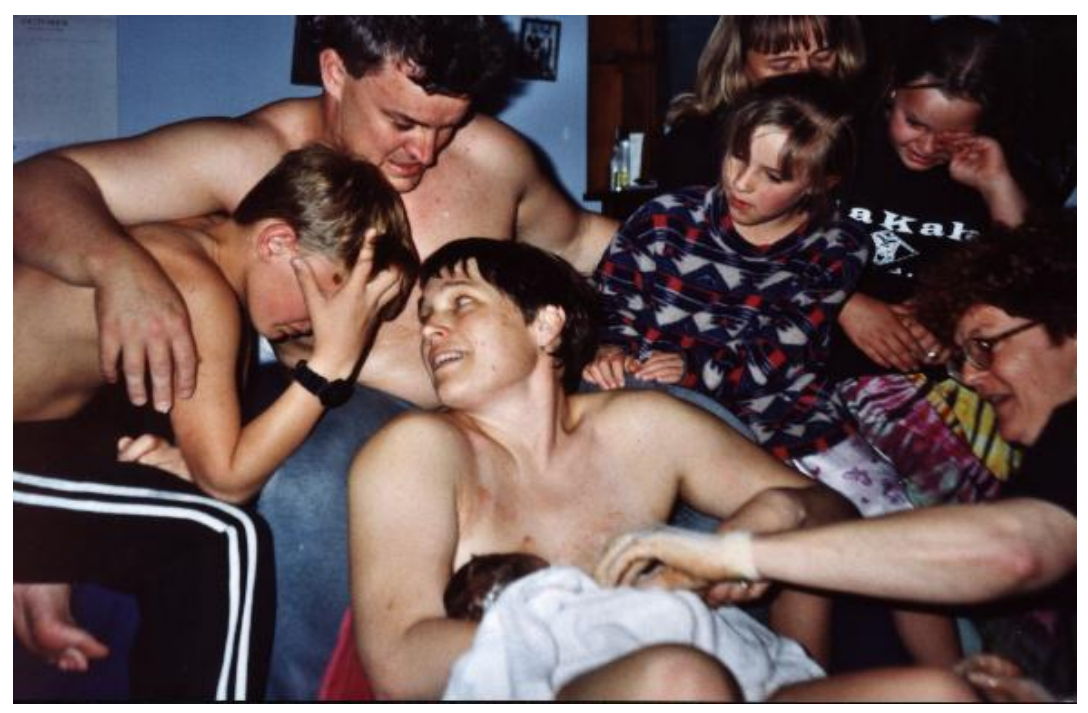

Figure 1.1 The birth at home of Hana Olds

(Reproduced with the permission of Bronwen and her family)

This is Hana, just a few seconds after she was born at home. Hana and her mother, Bronwen are surrounded by family and friends. Luke who is to the left, the one crying, is Bronwen's first born. He was born by emergency caesarean section at 34 weeks gestation. Bronwen's membranes had ruptured prematurely and she developed a uterine infection. Her second baby was born at home. During her third pregnancy Bronwen developed gestational diabetes but decided to continue with her plans for a home birth. By this stage she had accumulated several 'risk' factors: previous caesarean section and gestational diabetes, both of which are generally considered to preclude the decision for home birth. She booked me as her midwife for this, her fourth pregnancy and was looking forward to another successful home birth. Bronwen and her partner felt very ready to proceed with this plan. During the previous pregnancies they had thoroughly investigated the implications of the risk factors associated with these issues. They had had both midwifery and medical support (although tentative) for their decision and acknowledged that they may have needed to transfer to hospital. This fourth birth occurred nine years later. During this time, midwives in New Zealand had regained autonomy of practice, which meant that 
medical supervision was no a longer a legal requirement of care. I should also add that in this nine years the 'risk' environment had changed considerably.

Bronwen and her partner are articulate and well educated. Bronwen has a Masters Degree in Education. She is thoughtful, well-read, articulate and clear about her ability to make her own decisions about her birth. She is a keen user of alternative health therapies. During the pregnancy we had several conversations about the risks associated with home birth in light of her obstetric history. We also discussed my own risk given that I was agreeing to support a decision considered well outside the normal parameters for a home birth. Although in New Zealand there are no official guidelines on who is eligible for a home birth, women who have had a previous caesarean section and/or gestational diabetes would be commonly considered as being too 'at risk' to give birth at home. After extensive discussion and information sharing I recommended that she have a consultation with an obstetrician. She was happy to do this, mainly for my own risk management. Although the obstetrician clearly did not approve of her decision he did acknowledge her wishes. He recommended that should her diabetes be difficult to control or should she go past 38 weeks gestation she would need to revisit him and reconsider her birth plan. Bronwyn decided to go ahead with a home birth and I decided to support her. We had acknowledged each other's risks and respected each other's place. We had a clear and well-understood plan of care. Bronwen remained clear and focused. She maintained good control of her diabetes, went into labour at 38 weeks gestation and proceeded to have a normal labour and delivery at home. 


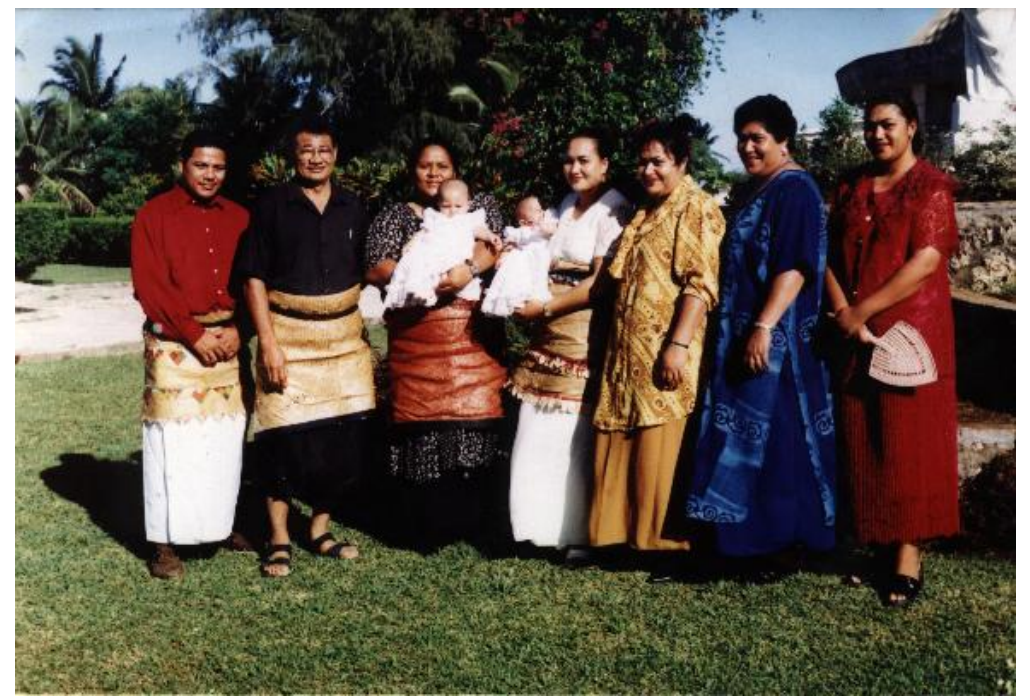

Figure 1.2 The christening of Alakoka and Fangaifua in Tonga

(Reproduced with the permission of Mele and her family)

This second photograph is taken in Tonga and was gifted to me by Mele, the mother of Alakoka and Fangaifua. Mele is the woman in white and the babies are her twin daughters. Alakoka and Fangaifua were born by caesarean section after Mele's attempted induction of labour at 38 weeks. There was no clinical indication for the induction other than a twin pregnancy. Both babies were well as was Mele. Mele never established labour so her caesarean section was undertaken for what is called 'failure to progress'. Mele is from Tonga and English is her second language. She has a large and supportive extended family. I had previously cared for both her sisterin-law and her cousin and she was keen for me to be her midwife too. Twins were discovered early in the pregnancy and I referred Mele for a consultation with an obstetrician to assist in providing her care. I was not able to attend this consultation. He advised her that she must transfer her total care from me to the secondary care clinic at the hospital, as this pregnancy was 'high risk'. She was told the hospital midwives would now look after her. She agreed and signed over her care to the hospital team, happy to take the recommendation of the doctor 'for the safety of her babies'. Mele was then seen by a different obstetrician at each visit, had no midwifery input planned and was cared for by five different and previously unknown midwives during her induction of labour. 
I had re-contacted Mele during the course of her antenatal care to make sure she was happy with what was happening and to confirm that I was available to provide the postnatal care once she was 'handed back' to me on discharge. When I spoke with her she was clearly upset over the care that was being provided. She was confused over plans for her care and distressed that she didn't have a midwife. She was cross with me for abandoning her and couldn't understand why I couldn't keep caring for her as well. From that point I kept in contact with her regularly, attended several antenatal visits with her and was a support person at her birth. She was keen that I go into the caesarean section with her. Ironically although I had been excluded from all aspects of her care, as it was deemed to be beyond my scope of practice, I was asked to be available in the operating theatre to resuscitate the babies as the paediatrician was delayed! Following the birth I made daily visits to her in the postnatal ward where she had a prolonged stay due to a significant uterine infection. As Mele had signed over her care to the hospital I received no payment for any of the visits I provided once this had occurred. After she was discharged home I had Mele's care 'handed back' to me and continued to visit her until her babies were six weeks old.

\section{Reflections on the stories}

Midwives are good at telling stories. Tears are shed, laughter is had and much is learnt in the telling and retelling of stories. These two stories that I have told are not atypical. They encapsulate midwifery practice in contemporary New Zealand. They illustrate the challenges we face and are the impetus for this research. Both of these experiences affected me deeply. Both were challenging. They both touched issues of consent, of choice, of agency, of relationship, and of the scope of practice of the midwife. They challenged for me the notion of what midwifery is all about and how we are best to practise in our world. I felt along the way, pride and shame, respect and doubt, powerless frustration and courageous triumph, all are manifestations of love and fear. What was revealed most to me in these stories was the importance of risk in the lives of women and of midwives.

For Bronwen and her baby there were the risks associated with her previous caesarean section and her gestational diabetes. The risks were uterine rupture, shoulder dystocia, hypoglycaemia and sudden fetal death. There were also risks if she had her 
baby in hospital: induction, caesarean section, formula feeds for the baby, iatrogenic infection, emotional dislocation and distress. How were we to calculate and balance these risks and who had the ultimate power to make the decision about where and how she was to give birth? For Mele and her babies there were also risks: induction, obstructed labour, caesarean section, postpartum haemorrhage, infection, fetal compromise or death. Who made her risk decisions? She followed the advice of the obstetrician and neither she nor I were active partners in that decision. As a Pacific woman there were the added factors of language and cultural difference and a difference in the perception of, and trust in the professionals. In the event Mele did suffer many of these interventions and complications. Although both babies in the end were well, Mele had been unhappy and socially disconnected. She also went on to need another caesarean section for her subsequent baby.

And then of course there was my own place as the midwife for these two women and my own perception of the risks. I have seen what happens when a uterus suddenly ruptures or when the baby's shoulders become impacted. I could anticipate the horror and know how much better it would be to be in hospital should this happen. Should I, or could I have communicated this to Bronwen? How risky was it really and how was I to manage my own anxiety? In Mele's case I might ask whether or not I belonged with her? Should midwives only stay focused on normal birth and leave complex or 'at risk' birth to obstetricians? What was important for Mele? And finally there was the impact of the medico legal environment. In going outside the recommended practice to support women such as Bronwen to deliver at home was I putting myself at risk. How was I to manage this? Did I have the knowledge and skill necessary to continue being involved in Mele's care?

What is also clear in these stories is the complexity of midwifery. Nothing is ever simple. We provide care for women who have many different perspectives and needs. All must be taken into account. We also need to collaborate with other health professionals, all of whom have their own perspectives. And of course we work in a complex social and cultural environment. All this is anything but simple. We have multiple arenas of accountability: the woman and her family, our peers, the maternity provider institutions, the community. There are also issues of power and control. The midwife needs to consider whose knowledge is seen as authoritative and who has the 
power to make decisions. The midwife weaves her care in ways that best meet the needs of the woman while at the same time acknowledging the demands of the environment in which she works.

\section{The research question}

There are many questions raised and issues highlighted in these stories. These issues are ones that are pivotal to the way midwifery and maternity care are currently constructed in New Zealand. These issues include: the role and scope of autonomous midwifery practice, the place of the referral for obstetric consultation, the nature of collaboration, the rise in intervention rates, the importance of informed choice and consent, and the impact of the medico legal environment. All these can be related to risk and to the way we manage it. This research then looks to investigate the relationship between love and fear in the lives of midwives: in how they feel, in how they talk, in what they do and in the consequences. Risk is chosen as the central concept of concern. The research question is therefore related to risk and how it is perceived and acted on. Risk, however is a rather abstract concept and difficult to investigate in isolation. Therefore I propose, for the purposes of this research, that risk can be examined most clearly in the processes of the referral for obstetric consultation. It is here that the midwife operationalises risk; she identifies and acts upon it. It is also the first intervention. No other significant birth interventions occur without it. It is also an unexamined intervention. Little is known about the rate and reasons for referral for consultation, the impact it has on further intervention, and even less about how midwives feel about it. In exploring risk, as expressed within the referral for obstetric consultation, this research is imbedded in the real day-to-day work of the midwife. The weave of midwifery can be examined in all its complexity, without leaving it unravelled.

The research question then is: How do midwives express and manage risk in their practice? It examines this question by identifying and investigating the referral for obstetric consultation as the place where risk is most clearly identified, not only risk to the mother and her baby, but also risk to the midwife herself. 


\section{Midwifery and maternity care in New Zealand}

This research is set in the context of New Zealand midwifery in the year 2000, and the group of midwives who are the participants in this research are those who provide continuity of midwifery care. In order for the reader to understand the processes and interpretation of the research, the context in which it is set needs to be made explicit.

\section{New Zealand and its childbearing population}

In 2000 New Zealand had a population of 3,800,000 and 56,605 births. The average age of childbearing was 29.3 years (Statistics New Zealand, 2004). Nineteen and a half percent of women were Maori, 10.1\% were Pacific women and 6.6\% were Asian (New Zealand Health Information Service, 2003). The population of New Zealand is spread between the North and South Islands. The majority of whom (76\%) live in the North Island (Statistics New Zealand, 2004). There are six hospitals which provide the full range of services including intensive care neonatal facilities and care for acutely ill women. These are based in New Zealand's major cities and are called tertiary facilities. Forty four percent of women who birth in hospital give birth in one of these tertiary facilities. A further $40 \%$ give birth in the country's 18 secondary facilities, which have caesarean section capability and newborn baby units. Only $16 \%$ of women give birth in the country's 64 primary birthing facilities (New Zealand Health Information Service, 2004). The large number of maternity units is a reflection of a population, which although small, is sparsely spread and set in mountainous geography. The number of women who plan to give birth at home is unknown.

\section{Birth outcome}

One of the most concerning trends in current maternity care is the escalating rate of obstetric interventions (World Health Organisation, 2004). Despite the fact that women, in the Western world at least, have never been healthier and that giving birth has never been safer we have seen a startling rise in the rate of caesarean sections, with little if any improvement in perinatal mortality rates. New Zealand is not an exception to this trend. Despite over a decade of independent midwifery the rate of caesarean section in New Zealand continues to rise. By 2002 it had reached 22.7\% (New Zealand Health Information Service, 2004). This rate is particularly 
concerning for midwives as we see that our area of expertise is in supporting normal birth (Donley, 1998; Kent, 2000). It is particularly concerning for New Zealand midwives. One might ponder over the reasons for this rise despite the growth of a strong and autonomous midwifery profession. Various authors have proposed that a focus on midwifery would be an effective way to counter this trend as midwifery care has been shown to reduce birth interventions (Blanchette, 1995; Kent, 2000; Lane, 1995; Page, 1995; Tew, 1995). In New Zealand it has been sobering to see that this is not necessarily so. What we are now beginning to understand is that rising intervention rates have causes that are likely to be complex and difficult to challenge.

\section{The recent history of New Zealand maternity care}

Maternity care in New Zealand as it currently stands is of recent duration. Changes to the Nurses Act in 1990 enabled midwives to return to autonomous practice. Concurrent changes were made to accompanying legislation and regulation allowing midwives to have prescribing and referral rights, access to laboratories and radiology services, access to hospitals and direct entry midwifery education.

Prior to 1990 midwives were very close to becoming extinct. Midwives could only work under the supervision of a medical practitioner and they were required to be nurses before becoming midwives. Following the North American model it was proposed that midwifery should become a postgraduate nursing certificate in maternal and child health and that the midwifery register should be discontinued. Midwifery was seen in a supportive and subservient role. Except for a very small number of home birth midwives, they provided no continuity of care. Nurses controlled midwives conditions of employment and midwifery education (Donley, 1986; Papps \& Olssen, 1997). Since 1990 maternity care in New Zealand has undergone dramatic change. One of the first questions a pregnant woman will be asked by friends and family is "Who is your midwife?" Midwives in New Zealand can now claim the place as the main maternity care providers. Over the last 10 years they have moved out of hospital employment in large numbers to work 'independently' in the community.

\section{The current organisation of maternity care}

In New Zealand maternity care is considered as primary health care. It is based on the premise that the needs of the childbearing woman are at the centre of care and that 
continuity of care is important (Ministry of Health, 1996). When women in New Zealand become pregnant they are required to choose and register with a Lead Maternity Caregiver (LMC). The LMC is the cornerstone of maternity care in New Zealand and can be a midwife, a General Practitioner or an obstetrician. At the time this research was undertaken $70 \%$ of women chose a midwife, $16 \%$ chose a General Practitioner and 14\% chose an obstetrician (New Zealand Health Information Service, 2003). By 2002 the proportion of women who chose a midwife had risen to $73 \%$. Women who registered their care with a GP had declined to $10 \%$ and those who chose an obstetrician had declined to $11 \%$. Six percent of registrations at that time were to unknown LMCs (New Zealand Health Information Service, 2004) ${ }^{1}$. A woman's LMC is responsible for assessing her needs, planning her care with her and ensuring the provision of maternity services. The LMC must be a named person, not a team or an institution as the woman must be able to build a relationship with her caregiver. Women now expect to be able to choose their LMC and expect and are expected to make active choices about the care they receive. Whether they give birth at home or in hospital they expect their known LMC to be there. These changes to the maternity system were profound and rapid. Women quickly became used to 'knowing their midwife' and they liked it. By 1999 women's satisfaction with their care was reported to be high. Over $75 \%$ and often, over $80 \%$ of women reported as being satisfied with their maternity care (National Health Committee, 1999).

While there are some discrepancies in the figures available about how many midwives were practising in New Zealand at the time of this study, analysis of these figures gives an indication that there were about 2000 practising midwives at the time, about 930 of whom provided LMC care on a self-employed basis. An unknown number of these 930 midwives were providing only post-natal care. There were estimated to be around 900 midwives providing a full LMC service throughout the whole pregnancy. The remaining midwives were employed in hospitals, or as managers, educators, researchers or policy advisors. An added complication to the interpretation of the figures is that some employed midwives also provide an LMC service through their employing institution. Such discrepancies mean that there is no way to accurately

\footnotetext{
${ }^{1}$ New Zealand's perinatal data collection has failed to collect up to $30 \%$ of data in some fields due to the fact that those not providing care under the Section 51 and subsequent Section 88 contract were not required to report data in all fields (New Zealand Health Information Service, 2003, 2004).
} 
identify the numbers of employed midwives who provided an LMC service or whether or not they are included in the 900 LMC midwives (Health Funding Authority, 2000; New Zealand Health Information Service, 2001, 2003). I return to this issue in Chapter 4 of the thesis.

Midwives who do not provide an LMC service work in hospital delivery units, antenatal wards or postnatal wards, working a shift system. They have become known in New Zealand as 'core' midwives, refecting the value placed upon retaining skilled midwifery care beyond the LMC system. In the delivery units 'core' midwives provide support for the LMC midwives, provide the midwifery care when the LMC is a doctor (the doctor must pay the hospital from his/her LMC funding when this occurs), and are available for secondary and tertiary assistance when required. It is of prime importance that there is a midwife present at every birth in New Zealand and this continues to be the case.

\section{Funding}

With rare exceptions, maternity care in New Zealand is fully funded by the State. An exception is made for obstetricians working in a private capacity who are permitted to make a charge over and above their State funding when acting as the LMC. There are also several private hospitals in the Auckland area that offer limited birth services. However, as there is no insurance cover available for maternity care in New Zealand, the consumer must pay the full cost of this service and it has only a minimal uptake.

In general then all LMCs are funded by the State to provide maternity care and are not permitted to make any additional charge. All LMCs sign a contract with the State to provide this care. This contract was instigated in 1996 and was known as Section 51 of the Health and Disability Services Act (Ministry of Health, 1996). There were some minor modifications made in 2000 and it became known as Section 88 of the New Zealand Public Health and Disability Act (Ministry of Health, 2002). Each birth is allocated a certain amount of funding on a modular basis, and this is maintained by the LMC in order to provide maternity care as detailed in the contract. Should the LMC wish to share part of the care with another provider, they are responsible for paying that provider out of their allocated funds. Midwives and doctors who are LMCs receive identical payments. Secondary and tertiary maternity services are 
funded differently. Funds are allocated to each of the 21 District Health Boards throughout the country according to the size of the population in their area. Primary maternity care however is funded centrally. The LMC sends an invoice to the central funding body on the completion of each care module.

\section{Professional organisation and education of midwives}

In 1988 New Zealand midwives developed their own professional organisation, the New Zealand College of Midwives (NZCOM), which initially focused its activities on supporting autonomous midwifery practice. It developed organisational structures that facilitated funding arrangements, developed standards for practice and a quality assurance/professional development programme. It is now a powerful advocate and supporter of midwifery and is represented at every local and national forum relevant to maternity care. It continues to act as a political voice to counteract the inevitable backlash from doctors, caught unprepared when the Nurses Act changed in 1990. It has been kept extremely busy. Along the way it has kept its focus on the normality of the childbearing process and on the role of the midwife in supporting birth as a normal healthy life event in partnership with the woman and her family, and with continuity of caregiver (New Zealand College of Midwives Inc, 1993).

Midwifery education in New Zealand is now undertaken as a three year, direct entry degree course from one of the country's 6 schools of midwifery. Most new midwives are not now nurses although the majority of midwives currently practising are also registered nurses (New Zealand Health Information Service, 2001). Four universities offer midwifery education to Masters and Doctorate level.

\section{The rights of the health consumer}

There are two particular aspects of the New Zealand social context that also need to be presented in order to place this research in context. The first relates to the role that the consumer plays in decision-making in health care. The most significant event in New Zealand relating to the role of consumer rights in health care was the release of the Cartwright Report, which investigated a controversial study of cervical cancer (Cartwright, 1988). The report revealed that the women in the study were recruited without knowledge or consent. They were randomly allocated into groups, one of which was a 'no treatment' group. There was poor monitoring of this research and it 
resulted in a considerable number of unnecessary fatalities. The release of this report and its recommendations had considerable impact on how research was to be conducted. More importantly it led to a profound change in the role of the consumer in health care. It resulted in the establishment of a Health and Disability Commissioner, charged with promoting the rights and responsibilities of consumers and resolving their complaints. It also led to the establishment of the Code of Health and Disability Services Consumers' Rights. The Commissioner and the Code are now the primary vehicles for dealing with complaints related to health care in New Zealand (Health and Disability Commissioner, 2004).

The second important aspect of New Zealand society to consider is the place of the Treaty of Waitangi. This treaty was signed between Maori, the indigenous people, and the Crown in 1840. Its principles are partnership, participation, protection and equity (Guilliland \& Pairman, 1995). In the last two decades there has been resurgence in its importance and the principles of the treaty are now enshrined in every State run service and in every piece of regulation.

These two aspects of New Zealand society have led to the role of the consumer as central to the decision-making about health care and to an understanding of the importance of needing to work in partnership. Partnership is now a key philosophical position of the midwifery profession (Guilliland \& Pairman, 1995). It is enshrined in midwifery's Code of Ethics and Standards for Practice (New Zealand College of Midwives Inc, 1993). Consumers are participating members of the New Zealand College of Midwives and also participate in annual reviews of midwifery practices. This partnership relationship as expressed in the New Zealand model has been interpreted and critiqued in terms of professional friendship (Pairman, 2000) professional accountability (Fleming, 2000), birth outcomes (Calvert, 2002) the realities of practice (Skinner, 1999) and in the light of power relations (Freeman, Timperley, \& Adair, 2004; Lauchland, 1996). It has not been critiqued with reference to the concept of risk and its management. 


\section{The medico-legal context}

A further area of the New Zealand context needs to be described when looking at midwifery care from a risk perspective. This is the medico legal context and in New Zealand this is complex. New Zealand, despite its 'no fault' accident and injury compensation scheme, has not escaped the escalating international trend to hold individual health practitioners accountable for adverse outcome. Despite an environment in which the consumer cannot sue either the practitioner or the institution, there continues to be an environment, reflected in the media, of a 'name, blame and shame' culture. There are also multiple arenas in which a midwife can be held accountable. In order of seriousness they include; the NZCOM internal Midwifery Standards Review Process, hospital clinical governance mechanisms, the police, the Coroners Court, the Accident Compensation Corporation, the Health and Disability Commissioner and finally the Midwifery Council ${ }^{2}$. The completion of these processes can take years. One of the most feared arenas however is the media. Midwives often cite this as a justification for being scared about the apparent risks of childbirth, to themselves. Anxiety levels about the risk of being investigated seem to be high. Although there have been studies of how negatively this environment impacts on New Zealand's doctors (Cunningham, 2004, 2004, 2004) and on midwives and doctors internationally, (Aslam, 1999; Henderson, 2001; Keaney, 1996; Symon, 1998; Symon, 2000; Wilson \& Symon, 2002), the degree to which this might impact on midwifery practice in New Zealand has not been investigated.

Data from the Health and Disability Commissioner (HDC), the Accident Compensation Corporation (ACC) and the Nursing Council reveal the frequency with which midwives are found accountable for substandard care. The HDC data show that, in the year this study was conducted there were 12 midwives who were found in breach of the Code (Table 1.1).

\footnotetext{
${ }^{2}$ The Midwifery Council of New Zealand was established in September 2004. Prior to this midwifery registration and education was controlled by the Nursing Council which had some midwifery membership.
} 
Table 1.1 Complaints made to Health and Disability Commission and breaches to the Code of Health and Disability Services Consumers' Rights

\begin{tabular}{llll}
\hline & $2001 / 2002$ & $2000 / 2001$ & $1999 / 2000$ \\
\hline Complaints & 30 & 43 & 28 \\
Code breached & 12 & 12 & 7 \\
\hline
\end{tabular}

Data from ACC in another report reveals that between 1996 and 2002 there were 28 midwives found in error and that in the same period 5 midwives had been disciplined by the New Zealand Nursing Council (Taylor, 2004). During this time midwives attended 390,000 births. What then is the risk of litigation? In order to calculate this the data from the HDC is used as all complaints to the Nursing Council (now the Midwifery Council) are referred to this body for initial investigation. In 2000, the year of this study, there were 43 midwives investigated for breach of the HDC code and 12 midwives in that year who were found in breach of it. One was referred on to the Nursing Council for disciplinary action. There were 56000 births that year. At each birth therefore there was a 1 in 1300 chance of a midwife having an official complaint investigated, a 1 in 4700 chance of being found in breach of the code and a 1 in 56,000 chance of being called before the professional disciplinary body. The risk of litigation seems small. Yet when this figure is extrapolated out over the working life of a midwife things look different. Given an average caseload of 40 births per year over a working life of 30 years a midwife might attend 1200 births. There is therefore an almost certain chance of being investigated once in her lifetime, a 1 in 4 chance of being found in breach of the code and a 1 in 200 chance of going before the Nursing Council. This would justify the common belief that midwives can expect a complaint at some stage during their practice.

This concern with risk is also found within the health institutions themselves with a growth in institutional clinical governance processes and risk management strategies (Blandford \& Smyth, 2002).

\section{Referral for obstetric consultation - processes and problems}

This research identifies the referral for obstetric consultation as the place where the expression of risk is most evident in practice. This process is complex and requires a detailed explanation as to how it occurs. LMC midwives who are caring for women 
who have, or who develop risk factors in their pregnancies are required to refer the woman to an obstetrician for a consultation. This can happen at any stage in the childbearing cycle. The framework for making decisions about the need to refer is included within the New Zealand Public Health and Disability Act 2000. Section 88 of this act includes the specifications contract (Ministry of Health, 2002). These guidelines were negotiated and agreed to between midwives, general practitioners and obstetricians. The guidelines provide a comprehensive list of possible complications and rate these complications according to what type of action is expected. A level 1 referral in the guidelines requires that: The Lead Maternity Carer may recommend to the woman that a consultation with a specialist is warranted. A condition that is given a Level 2 requires that The lead Maternity Caregiver must recommend that a consultation with a specialist is warranted. A Level 3 condition states that The Lead Maternity Carer must recommend to the woman that the responsibility for her care be transferred to a specialist (Ministry of Health, 2002). Examples of conditions and related referral levels are provided in Table1.2

Table 1.2 Examples of reasons and levels of referral taken from Guidelines for Consultation with Obstetric and Related Specialist Services (Ministry of Health, 2002)

Condition Description

Level of

referral

\begin{tabular}{|c|c|c|}
\hline \multicolumn{3}{|l|}{ Antenatal } \\
\hline Diabetes & $\begin{array}{l}\text { Pre-existing (insulin dependent } \\
\text { or non insulin dependent) } \\
\text { Gestational, well controlled on } \\
\text { diet }\end{array}$ & 2 \\
\hline Previous caesarean section & & 2 \\
\hline Multiple pregnancy & Twins or higher order multiples & 3 \\
\hline Reduced foetal movements & & 1 \\
\hline \multicolumn{3}{|l|}{ Labour } \\
\hline Epidural & & 2 \\
\hline Prolonged first stage of labour & $\begin{array}{l}\text { Nullipara - poor progress after } \\
\text { ARM and syntocinon }\end{array}$ & 2 \\
\hline Pyrexia in labour & $\begin{array}{l}>38 \text { degrees with or without } \\
\text { foetal tachycardia }\end{array}$ & 2 \\
\hline \multicolumn{3}{|l|}{ Postpartum } \\
\hline Breast abscess & Not settling with antibiotics & 2 \\
\hline Puerperal sepsis & $\begin{array}{l}\text { Temp }>37.6, \text { maternal } \\
\text { tachycardia }\end{array}$ & 3 \\
\hline
\end{tabular}


This document retains a focus on women being at the centre of decision-making. It states,

Where a consultation occurs, the decision regarding ongoing clinical roles/responsibilities must involve a three-way discussion between the specialist, the Lead Maternity Carer and the woman concerned. This should include discussion on any need for and timing of specialist review (Ministry of Health, 2002, p31).

LMC midwives in New Zealand make referrals to obstetricians at any stage. When the woman becomes 'high risk' clinical responsibility will be handed over to the obstetrician. The woman and the midwife are both meant to be involved in this decision. Nothing is known about how often any of this occurs and little is known about how successfully it works. What is known is that the LMC midwives are, to varying degrees, working in hospitals providing what is considered secondary care. They do this in collaboration with obstetricians and the hospital or 'core' midwives. The Health Funding Authority in 2000 stated:

Maternity should be considered a leader in integration. No other service has primary practitioners working within a secondary facility. The Guidelines for referral to Obstetric and Related Specialist Medical Services help to facilitate this integration (Health Funding Authority, 2000, p59).

In the original Section 51 document there was a loophole, which meant that there was a financial incentive for the secondary service in having the care transferred. If the original LMC midwife did not continue care the hospital could claim the Section 51 primary care funding on top of the secondary service funding. LMC midwives and mothers in several areas were put under pressure from obstetricians in hospital clinics to hand over LMC. The Health Funding Authority's 2000 report on the New Zealand maternity service, recommended that this process be discontinued (Health Funding Authority, 2000). This recommendation became part of the revised Section 88 document in 2000. However there was not total compliance and the 2004 Maternity Report stated that some hospitals were still claiming the primary maternity funding 
for services provided to women whose care had been transferred (New Zealand Health Information Service, 2004). This would indicate that the interface between primary and secondary maternity care is not entirely straightforward and that in some areas at least the relationships are less than collaborative. My own experience of the interface in situations such as Mele's would confirm this. In this type of situation funding issues had influenced the obstetricians to collude with the management of the hospital in forcing handover of LMC status from the midwives, excluding them from continuing care. There is no information about the extent to which this has been happening.

To complicate matters further these guidelines provide for the possibility that the LMC midwife may continue midwifery care once the responsibility for care has been transferred. The document states, "There are some particular circumstances, for example twins, where clinically the specialist needs to be responsible for care but the ongoing involvement of the primary practitioner is very important" (Ministry of Health, 2002, p31).

What is not mentioned however is that the midwife who does continue care must inevitably retain the responsibility for whatever aspect of the care that she provides. It would be unreasonable and run counter to effective collaborative practice to insist that all responsibility be transferred to the obstetrician. This advice also relies on a trusting relationship between midwife and obstetrician. Where relationships are poor the boundaries of responsibility for clinical decision-making can be fraught and the negotiation about the ongoing involvement of the primary practitioner is very problematic. This is reflected in Mele's story and anecdotally in the stories of other midwives. This research investigates the degree to which ongoing care continues, once transfer of clinical responsibility has occurred.

Another variable to add to this mix is that the woman does not have to follow the advice of the midwife to have a consultation. This is not an uncommon occurrence but there are no data to indicate how often this occurs. Good collaborative care will ensure that the midwife is able to discuss this with the obstetrician and work together to ensure the best outcome for the mother. In situations were there are poor relationships the midwife can find herself caught in the middle, either supporting the 
woman at the expense of her professional relationships or not supporting the woman at the expense of her professional integrity.

\section{Summary}

Midwives provide support and assistance for women and their families at a critical moment in their lives. This research seeks to add to the understanding of how they make sense of this complex and challenging work, given the current socio-cultural context in which they work. Risk has been identified as a key concept driving maternity care and has been chosen as a theoretical framework for the research. It has been operationalised as the referral for obstetric consultation in order to find a 'way in' to the topic but also to keep the research connected to the every-day working world of the midwife. This is after all the work of a midwife.

\section{Structure of the thesis}

There is no way a work which seeks to investigate a topic such as risk could be undertaken without acknowledging its complexity, especially where current perspectives are reflective of an ever-increasing understanding of multiple worldviews and approaches. The overarching issue in this research is the place of risk in midwifery. It looks at this through multiple lenses, one of which of course is my own. Other lenses include a variety of theoretical and methodological viewpoints.

Chapter 2 begins this research by investigating some of the key theoretical approaches to risk in relation to this study. The lenses used reflect the continuum of perspectives on risk from the techno-rational approach, through to the socio cultural approaches of Ulrich Beck's 'risk society'(1999) and Mary Douglas's (1992) ideas of risk, danger and blame. At the other end of this spectrum are the post-modern approaches most powerfully presented in Foucault's (1991) work on governmentality. The chapter outlines these approaches with some ensuing discussion and relates this to midwifery and to the study.

In Chapter 3, I refocus the question in light of these theoretical lenses and describe the methodological underpinning of the research. As this study is a complex intertwining of ideas across the spectrum of knowledge I have used the philosophical approach of 
Roy Bhaskar (1993) and his interpretation of critical realism. This chapter then describes the design of the research, which uses two data collection methods; there is both a national survey and a series of focus groups

Chapter 4 provides the detail of the design and process of the first part of the research, which is a total population survey of LMC midwives. This survey requested detailed information on demographic data of both the midwives and the mothers they referred for obstetric consultation over a 4-month period in 2000. Detailed information is requested on each of the referrals including the reasons for referral and the degree to which the midwives continued to provide care after a referral was made. The final part of the survey is a series of questions related to the midwives' attitudes to the referral processes and to risk.

Chapter 5 presents the survey results and Chapter 6 discusses the implications of these results. It highlights the characteristics of the midwifery workforce, discusses the implications of the midwives' reasons for and patterns of referral and begins to reflect on these findings in light of risk theory.

Chapter 7 presents the details of the design, implementation and the process of data analysis of the six focus groups. These groups were held with LMC midwives in a variety of New Zealand settings. Chapter 8 describes and interprets the focus group data within the four constructed themes that emerged during the analysis. These themes are 'being with women', 'being professional', 'working the system' and 'working with complexity'. As these themes are being presented, the relevant findings of the survey are reintroduced. In this way the two views of the research can support each other and add depth. In a sense then the data can be viewed in a binocular way. The analysis having been completed it is then reviewed in light of risk theory.

In Chapter 9 these themes are configured into a model of midwifery; a way midwives can make sense of their work as embedded in a 'risk society'. The model is a threelegged stool. The seat of the stool is 'being with women' and the three legs are the other three themes, 'being professional', working the system' and working with complexity'. The struts of the stool I have called 'story telling'. This is a birth stool for the midwife not for the mother. This stool is then described as a tool for supporting the midwife in her work and reflects on other current midwifery research. 
The discussion within this research is therefore presented at a number of levels. The first level reflects the quantifiable processes of referral for obstetric consultation as a place where risk is expressed. The second level reflects the experiences, values and attitudes of the midwives and incorporates the results of the survey data. And finally the model development is presented. The model describes and interprets the midwives' relationship 'with risk', drawing together all the findings of the study including the survey, the focus groups, and the theoretical approaches. It is set within the current socio-cultural context. The thesis concludes with a reflection on the research process itself and with a number of recommendations that have emerged. 


\section{Chapter 2: Risk Theory}

\section{Introduction}

Risk impacts on the lives of midwives both in the assessment of risk in the women we care for, and in the management of our own risk within the current medico-legal context. As it is currently expressed, the risk paradigm directly challenges the model of birth as a normal part of human existence and presents challenges for midwives as we attempt to put this model of normality into practice. Midwives are faced with a significant paradox in attempting to work a 'birth is normal' paradigm within a 'birth is risky' context. I propose that risk in its current construction contributes significantly to increasing intervention and escalating medico-legal action, and is a core issue for maternity care in general and midwifery in particular. There appears to be an intensification of anxiety around childbirth despite a growing understanding about the causes, incidence and prevention of negative outcomes. This has been accompanied by a growth in the medico-legal environment requiring increasing levels of accountability and surveillance. It feels as if we are more tightly controlled than ever. What does this mean for the midwifery? How does 'risk' affect our practice and how do we make sense of it?

This chapter investigates some of the theoretical perspectives on risk that might inform the answers to these questions. There are two particular theoretical perspectives that I address. The first relates to the techno rational aspects of risk and looks at the development of the science of probability and the implications this has had on the notion of 'normal'. The second relates to the sociocultural aspects of risk. The work of two key risk theorists Ulrich Beck $(1992,1999,2000)$ and Mary Douglas (1992) are presented. I then briefly address some of the ideas associated with the post-modern position particularly the idea of governmentality in the context of the work of Foucault (Foucault, 1991; Turner, 1997). These positions are summarised in Table 2.1. Although these perspectives on risk are presented as separate entities in this chapter, they are anything but separate in the working world of the midwife where they interact in complex and often conflicting ways. This is reflected in Bronwen's and Mele's stories, as described in the previous chapter. Who identified 
the risks that required attention, who decided how the risks could be quantified, and whose values determined the priorities for action, were all important facets in the care for each woman. I will return again to Bronwen's and Mele's experiences when reflecting on the application of the theoretical approaches to risk.

Table 2.1 Theoretical perspectives on risk

\begin{tabular}{ll}
\hline Theoretical perspectives & Key issues \\
\hline Techno-rational & Risk is real and can be measured objectively. \\
& How do we measure risks? How do we measure how \\
& people respond to risks? How do we avoid and control \\
& risks? How do we bring deviant behaviour into line \\
& with what we measure as normal?
\end{tabular}

Sociocultural

'Risk society'

'Cultural/symbolic'

Post modern
Risk is real but is always mediated through social and cultural practice

How is the expression of risk related to the structures and processes of current social form? How are risks understood in different sociocultural contexts?

Why are some dangers selected as risks and others not?

What is the situated context of risk?

Nothing is a risk in itself. Risk is a product of history, society and politics. What are the discourses and practices of risk behaviour?

Adapted from (Lupton, 1999, p35)

\section{Techno-rational approaches to risk: chance and probability}

The most dominant discourse related to risk is found within the techno-rational approach. This approach sees risks as real and seeks to control or avoid them. It focuses on the mathematical calculations associated with the determination of the probability of an event occurring in a population. The primary interest is in the accuracy of the predictions and in the quantifiable measurement of outcome. Although most practitioners who work in this paradigm do acknowledge that risk assessment is not value-free, these assessments tend to be treated as if they are objective and rational (Lupton, 1999). To return to Bronwen and Mele's situations as examples, the risk of complete uterine rupture in labour when there has been a lower segment caesarean section in a previous pregnancy has been estimated to be between 0.2 and 1.5\% (New Zealand Guidelines Group, 2004). Smith, Pell and Dobbie's (2002) retrospective cohort study of twin delivery found that there was an absolute risk of perinatal death for second twins born at term of 1 in 270 for all causes. This 
can be helpful information for the practitioner and for the woman. How this information is assessed and then communicated to the individual woman and how decisions are then made however is subjective and value-laden. In Bronwen's case she decided that the risks were small enough to justify delivering at home. Others would not have thought that it was worth the risk. Mele was not given this information. Nor was she part of the decision to have her labour induced two weeks before her due date. She was quite happy for the obstetrician who 'knew best' to make the decision for her.

\section{The role of numbers and a place for normal}

The different ways Bronwen and Mele felt about involvement in the decision-making poses a question about cultural understandings of probability and risk. Mele seemed more fatalistic and deferred to the expertise of Western medicine whereas Bronwen felt the need to engage in the management of her risk. This need to 'manage' risk has its origins alongside the development of modernity, a place more culturally associate with Bronwen's rather than Mele's background. From the Western perspective, prior to the $18^{\text {th }}$ century, how long one lived was essentially a matter of chance. It was thought that life was predetermined and the Gods played a key role (Hacking, 1990). During the $18^{\text {th }}$ century theorising about probability began and from that point the changing nature of probability and its relationship with risk became defining features of the Western world (Heyman, 1998). Central to this transformation was what Hacking called the 'avalanche of printed numbers'. As the world became numerical we began to count populations and their characteristics, undermining the notion of determinism. It was seen that humans might be able to have some control over their destiny.

The counting of people and their habits led to the development of the concept of the 'normal' person by the late $19^{\text {th }}$ century. The Belgium astronomer and mathematician Adolphe Quetelet (1796-1874) established that the distribution of naturally occurring phenomena follows a 'normal curve' (Oakley, 2000). He built on the perspectives of Hobbs, Condorcet and Laplace, applying their ideas on probability to the measurement of uncertainty in the social sciences, and proposed that mathematics was an appropriate tool for the study of society (Menand, 2002). His major contribution 
was the notion of normal distribution, the creation of the bell-shaped, normal curve, and the concept of 'the average man'.

Underlying this growth in the categorisation and numeration of the population and the development of the idea of the 'normal man' was the concept of deviance, initially applied to the criminal, the insane and the ill, but increasingly applied to wider sections of the community (Hacking, 1990). In the case of Quetelet's normal curve, deviations from the normal are as predictable as the norm itself (Menand, 2002). This ability to identify and particularly to quantify the deviant had a decidedly judgemental aspect and was associated with the control and management of deviant populations. What was considered 'not normal' was considered pathological (Hacking, 1990). As the condition of normal was included in almost every sphere of life, the meaning of normal came to mean 'usual'. The descriptions themselves were an impetus for change as people were then expected or even forced, to become 'normal'. This term is still a part of statistical language expressed as the standard deviation. Lupton (1999) comments that in current Western societies, where controllability has become increasingly important, the idea of 'risk' and the lack of control over the outcome, have become associated with deviations from normal. Uncontrollability itself has become risky. The notion of normal then over this time underwent a fundamental change from being a social to a scientific concept as we came to accept the idea that one can't know something unless it can be measured (Hacking, 1990).

Hacking proposes that the development of the science of probability can be seen as an incredible success story, not only within epidemiology but also within metaphysics, epistemology, logic and ethics. He says that although the measurement of probability itself cannot determine what we should do or what we should value, it has had an enormous impact on decision-making at every level.

Probability cannot dictate values, but it now lies at the basis of all reasonable choice made by officials. No public decision, no risk analysis, no environmental impact, no military probabilities can be conducted without decision theory couched in terms of probabilities. By covering opinion with a veneer of objectivity, we replace judgement by computation (p4). 
$\mathrm{He}$ is indicating that it is the measurable assessment of risk that has now come to dominate how we live our lives and what we value, especially in the public arena.

\section{Probability and medicine}

Probability and risk assessment are central to the medical discourse. Medicine has a focus on mathematics and an interest in quantifying the distribution and the risk of pathology (or deviance from normal health). Probability and risk assessment are also widely used in medical research to establish the efficacy of treatment (odds ratios and relative risk). What is less well known however is that this link between mathematics and medicine is of long standing. Medicine was at the forefront in the development of statistics and has been used right through the $20^{\text {th }}$ century as an exemplar of how quantification and precision ought to be used in research and in policy (Oakley, 2000). Quetelet himself applied his early statistical ideas to medicine. He asked how doctors could possibly know how ill someone was if they didn't understand the health of the 'average man'. Probability and medicine then share a long history together. By the early $20^{\text {th }}$ century medicine had changed from art to science and its professional legitimacy had shifted from humanitarian healer to empirical scientist (Oakley, 2000). It is no surprise therefore that medicine should have taken the lead in the uptake and development of the 'evidence based practice' paradigm. Neither is it surprising that our modern understanding of normal has evolved within this medical context (Hacking, 1990). It is this bio-medical approach to normality that is particularly problematic for the midwife.

\section{Risk and normality - a quandary for the midwife}

Within medicine, normal is considered as the absence of measurably pathology. However just as one cannot accurately predict pathology neither can one predict normality (Murphy-Lawless, 1998). Through the bio-medical gaze the normal itself then becomes risky. Risks are identified and controlled through the use technology, monitoring and intervention (Cartwright \& Thomas, 2001; De Vries, 1996). This poses a significant quandary for the midwife attempting to act as the specialist or guardian of normal birth. Understandings of what is risky and what is normal both dominate and delineate midwifery practice and yet are often seen as juxtaposed positions. Midwives claim expertise in the normal, medicine in the 'at risk'. Medicine however claims that all birth is risky and that birth is normal only in 
retrospect (Wagner, 1994). Risk thus encroaches on and undermines the 'normal birth' work of the midwife.

It is within this dominant medical discourse that midwives claim to be the practitioners of normal childbirth. It is a precarious position to take given who is defining normality and who is defining risk. The challenge may be for midwifery to look beyond medicine's definitions of normal and to claim its own. What definition of normal does a midwife accept? Where is midwifery placed in its attitude to risk and to normality? For the midwife, is normal the absence of pathology, or does it consist of the average or usual?

Walker (1976) attempts to overcome the problem of definition by making a distinction between normal as natural, and normal as statistically common. This, she says allows the midwife to look after women who are deemed 'at risk' as they can have a birth without intervention, a 'normal' birth. More recently there have been attempts to avoid the problems associated with the word 'normal', by renaming normal birth as physiological birth (Walsh, El-Nemer and Downe, 2004). Cartwright and Thomas (2001) attempt to overcome the problem by proposing that there are 'normal' complications of birth, such as the need for an episiotomy or intravenous fluid. Thus they see that it is possible to view some pathology as normal or 'usual'.

The problem with these definitions of normal is that they attempt to overlook or bypass the dominant and authoritative discourse, that of medicine (Jordan, 1997). In attempting to define a scope of practice within the normal or physiological process the midwife will inevitably come up against the medical discourse, which claims the ultimate authority over birth. Medicine has managed to do this by colonising risk (Lane, 1995). People in Western cultures tend to defer uncritically to numeric understandings of risk (Heyman, 1998) and powerful occupational groups (such as doctors) can convince people of their ability to reduce risk. They may actually emphasise risk in order to do so (De Vries, 1996). Mander (2002) claims that obstetricians, in their need to maintain dominance over midwifery can undermine the confidence of the women both in herself as child bearer and in midwifery, as being able to care for her. She states, "It may be that the midwife has colluded in this 
medical strategy by over-emphasising the normality and healthiness of childbearing, thus possibly jeopardising her own role" (p185).

Risk, I propose, is therefore the central issue with which midwifery must deal in order to promote normal or physiological birth. Coping with risk while attempting to promote normality is fraught with difficulty as it challenges the dominant discourse. Being seen as promoting a normal birth discourse can make midwives vulnerable medico-legally and can make collaboration and communication problematic. Some midwives tend to avoid the problem by caring only for the normal or 'low risk' women as defined by medicine. The problem with this position is that as medicine develops its technology it can and does identify increasing numbers of women who require medical care. New technologies, particularly in the antenatal period discover an increasing number of 'complications' that medicine requires to be monitored and to have life-saving, prophylactic interventions (Katz-Rothman, 2001). As this happens the number of women who are 'low risk' and thus eligible for midwifery care decreases. The alternative position is to claim that all women need midwifery care regardless of risk. The problem with this position is that the midwife is herself at risk of becoming medicalised. Katz Rothman (1981) comments on this dilemma:

If midwives are denied the use of available technology, then they obviously won't have it when they need it. They will have to turn over every patient who needs assistance, or even diagnosis, to a physician. But if she's allowed the medical technology, then what prevents the midwife from becoming a 'mini-obstetrician' as critics have claimed (p160)?

This research seeks to explore the 'risk' problem. It examines midwifery practice to ascertain the extent to which midwives continue to provide care for women who are 'at risk' and uncovers midwives' attitudes to the way their scope of practice is defined. It offers important insight into how New Zealand midwives attempt to balance this 'risk versus normal' debate.

\section{A problem with probability - the ecological fallacy}

There is one further problem with the techno rational approach that needs to be considered. This is found within the paradigm itself. For the health practitioner, either midwife or obstetrician, there is a fundamental difficulty of relating population- 
based knowledge to individual situations. In attempting to reduce uncertainty, practitioners attempt to predict the likelihood of an outcome for an individual using aggregated data. However this flawed process is recognised as the ecological fallacy, which occurs when conclusions are drawn about individuals based on analysis of group data (Williams, 2002). What can happen is that the estimation is oversimplified, leading to systematic error (Heyman, 1998). The difficulty can be seen if we return to Bronwen's story. In order to estimate Bronwen's risk of rupturing her uterus for example, we can look at the data related to the incidence of ruptured uterus in a labour where, as in Bronwen's case there has been a previous caesarean section. However Bronwen had had two 2 normal births subsequent to the caesarean section, which must have decreased her risk considerably. She also had one of her normal births while she had gestational diabetes, which must then have increased her risk again. There was absolutely no way that Bronwen's risk of ruptured uterus could be estimated given the particular characteristics of her individual situation. It would have been fallacy to have told her that her risks were 1 in 10 or even 1 in 1000 . The same dilemma applied to Mele and her risk of having birth complications associated with her twin pregnancy.

The ecological fallacy is most evident when we examine risk screening. Formal risk screening refers to the process of allocating a risk score to an individual woman according to her risk factors. It is now widely accepted that individual risk factors are poor predictors of outcome (Rohde, 1995; Rooney, 1992) and that risk screening suffers from low sensitivity as well as low specificity (Saxell, 2000; Schettler, Solomon, Valenti, \& Huddle, 1999; Yuster, 1995). Interestingly the World Health Organisation (WHO), which had promoted risk screening in the 1980s as an effective way of combating maternal and perinatal mortality has now changed its policy. In its 1998 document the WHO states:

Risk assessment should not be relied on as the basis for matching needs and care in maternity services. It is almost impossible to predict, on an individual basis, who will develop a life-threatening complication. Sensitivity, specificity, and positive predictive value of risk assessments using such characteristics are poor (World Health Organisation, 1998). 
The WHO priority is now for the assessment of risk to be set in the context of a maternity service which is accessible, provides continuity of care and is well integrated and of a high quality. The WHO also states that early identification and appropriate referral and management of obstetric complications are vital. Part of the reason for the WHO change of policy was that it saw that risk assessment as a strategy was flawed and led to an increase in obstetric intervention with no improvements in maternal or perinatal outcome (World Health Organisation, 2004).

Midwifery must manage risk by using information from the techno rational paradigm in the protection of normal birth. What creates a tension for the midwife is the continued dominance of risk in the planning and provision of care. However the success story of probability within a modernist framework only partly explains current constructions of risk. Increasing use of technology and the quantification of life fails to explain on its own either the increase in anxiety related to risk and its management, or the considerable variation in risk perception and in decision making. For this we must turn to an analysis of risk from a sociocultural perspective.

\section{Sociocultural approaches}

It is apparent in Bronwen's and Mele's stories, that attitudes towards risk and how these impact on risk decision-making are heavily influenced by their sociocultural setting. Midwives must deal not only with risk as probability but also with risk as a qualitative entity. It is important to understand how and why risk decisions are made. There is now a growing body of literature in the social sciences addressing this. There are two major perspectives that I present. The first relates to how risk is expressed in the current social form and the second, how values impact on risk decision-making. I will also briefly address the postmodern position, which looks at the role of governmentality and surveillance.

\section{Risk society - a grand theory}

Beck (1999) tells us that we now live in a 'risk society'. We are anxious and fearful despite never having been safer. He traces our anxieties about risk back to the beginnings of modernity. With its origins in the enlightenment, modern life was characterised by collective patterns in which social roles were well established. We believed in progress, that we should be able to have control over our lives and that full 
employment was possible. Nature was there to be exploited. Technology would find a way of solving all our problems and science was seen as the perfect rational project. Beck states that these beliefs have been undermined in more recent times by five things: by globalisation in which we are forced to see and acknowledge multiple world realities; by individualisation and the gender revolution in which social roles and collective patterns of life are no longer preordained; by under-employment, and by the nature of global risk, both financial and environmental.

All these factors Beck proposes, increase the levels of global, societal and personal anxiety. The ideas of controllability and security have collapsed. People have lost faith in professionals and in technology. Not only can technology not solve all problems, it actually creates some of them. Science and industry itself are now held accountable for the production of risk. In a sense science has been affected by its own success. Progress has been overshadowed by the risks produced (Lane, 1995).

The risks we face are a new breed of risk as they are globalised (pollution, contamination of food, climate fluctuations). We are now, according to Beck, a 'world risk society'. He characterises the risk society as one in which we face the consequences of modernity's success. It is, in a sense, perfection realised. He states that the risk environment has made visible the impossibility of modernity's promises of eventual controllability and predictability. It disarms and unsettles us.

To the extent that risks become the all-embracing background for perceiving the world, the alarm they provoke creates an atmosphere of powerlessness and paralysis. Doing nothing and demanding too much both transform the world into a series of indomitable risks. This could be called the risk trap. One thing is clear: how one acts in this situation is no longer something that can be decided by experts. Risks pointed out (or obscured) by experts at the same time disarm these experts, because they force everyone to decide for themselves: what is still tolerable and what no longer (Beck, 1999, p141).

We are then, facing the consequences of the success of modernity and its assumptions are being challenged. This is very disconcerting, as we have not yet created societal and cultural structures to replace it. We are not yet, according to Beck, post-modern. 
We live a contradictory existence, and feel as a consequence, a great deal of anxiety. On the one hand we have lost faith in the experts yet on the other we still demand that the experts produce work free of any negative outcome. We strive more and more intensely to avoid or control risk even while knowing that we can never do this. In terms of maternity care, this reflexive culture of late modernity means that we as health practitioners can be constantly questioned and challenged both by the consumers and the managers of maternity care. The accountability that results causes fear and stress not only in the practitioners but also in the consumers. What can happen as a result is that the emphasis we place on avoiding negative outcomes can result in defensive practice and increased intervention.

Herein lies the irony, for the panic culture that emerges, and the negative backlash that it effects, is itself a product of the consumerism and new managerialism that seeks, in fact to achieve the opposite; that is, to enhance rather than undermine the quality of care that is provided (Annandale, 1996, p417).

It seems therefore that we are clinging to the precepts of modernity yet at the same time realising that modernity is failing us. We demand fully informed choice without realising that this is not possible. We assume that if negative outcomes occur then someone hasn't done their risk management well enough.

The way this 'risk trap' occurs in maternity care could be seen as paradoxical; failure of the midwife to produce the perfect outcome is seen as a personal failure often unrelated to the normal risks associated with birth. It seems the fact that babies sometimes die is a piece of risk evidence to be avoided. Medicine has been very successful in its colonisation of childbirth by focusing primarily on mortality, morbidity and on its own interpretation of risk. For example the World Congress of Perinatal Medicine, held in Barcelona in September of 2001, prepared a code named "The Rights of Mother and Newborn". One of the rights was that: "All women have the right to be guaranteed by the government of any country in the world a risk-free pregnancy."

Beck's thesis is therefore particularly appropriate for childbirth especially in the light of the increase in medico-legal activity, intervention, accountability and consumer 
pressure. Modernity, represented by the science of obstetrics has increased its knowledge and its technology in an effort to provide risk free birth. But as we see, this risk discourse comes with its own risk.

What is at issue now however is not the question of risk or no risk but i) the iatrogenic risk of medical interventions ii) the realisation of social control over women by the use of the risk vocabulary to describe maternity iii) what additional criteria should be used to assess risk... and iv) whether risk has monopolised the debates about childbirth to the exclusion of other factors such as emotional satisfaction and control over the events and procedures surrounding birth (Lane, 1995, p56).

One of the interesting things about this 'risk' society ... "is that it combines elements which used to be considered as separate - society and nature, social sciences and material sciences, the way we talk about risk and the reality of the threats that are posed (Beck, 1999, p4)". Risk, according to Beck, does not have a preference for any one form of knowledge. The implication of this is that risk analysis needs to have a multidisciplinary approach. 'It needs to include both a sociological and technological approach. It is a process fraught with complexity. Perhaps then this is part of the resurgence of autonomous midwifery in New Zealand. The world is acknowledging that medicine on its own cannot deal with birth. It is a complex and multidimensional project.

Risk society functions not only on a global level but also at the level of the individual. The individualisation that has occurred means that people are inevitably bound up in the risk world in their personal life and are expected to make their own risk decisions. One's culture and community are no longer seen as responsible for one's actions. The individual practitioner and the consumer of health care must decide for themselves what choice they will make between risky alternatives.

Attitudes to risk are also very much related to how fear is felt and expressed and how power is manifest (Giddens, 1999). We see this clearly in the decision-making processes around birth. Take for example a woman's decision to give birth to her breech baby without intervention as compared to an obstetrician's decision to deliver her baby by caesarian section, or a woman's choice to have an epidural anaesthetic as 
compared to the midwife's commitment to normal birth. Whose knowledge is authoritative here? How is fear being expressed? And of course, who is at risk?

With risk, in a sense, we can terrify ourselves into stagnation. Risk, Beck says begins where trust in our security and belief in progress end.

The concept of risk characterizes a peculiar, intermediate state between security and destruction, where the perception of threatening risks determines thought and action (Beck, 2000, p213).

What of the future of this risk society? Beck saw a fundamental shift occurring in the way society is being constructed as security and controllability collapses. He thinks that a fundamental paradigm shift is required.

A new kind of capitalism, a new kind of economy, a new kind of personal

life are coming into being, all of which differ from earlier phases of social development. Thus, sociologically and politically, we need a paradigmshift, a new frame of reference (Beck, 1999, p2).

\section{Summary of Beck's view of risk}

Beck proposes that our attitudes towards risk, as expressed in late modern society is in the process of undergoing fundamental change. We are caught in a paradox; beginning to understand that modernity is not able to control risk, yet at the same time still expecting it to do so. We are seeing how complex the world is, yet have no framework, no paradigm to deal with this. We have no simple answers anymore. Our attempts at certainty and controllability fail and as they do we strive more and more to control. This action paradoxically creates more risk, not less. Increasing interventions in childbirth are the perfect example of this. What does it mean for midwifery? Are midwives caught up in this 'risk trap' or can midwifery offer an alternative paradigm, a more comprehensive approach? This research aims to illustrate how midwives make sense of risk in practice. A study of midwifery in New Zealand is a good site for such research as we now have a large population of autonomous, community based midwives who promote (in their official standards at least) normal birthing. Childbirth is an appropriate site for the study of society. If you want to see how society is faring one only need look at how women are supported to give birth. To some extent one might say that the changes to childbirth services in New Zealand and a study of midwifery after a resurgence from near extinction at this 
moment in Western development might provide some valuable insight into how to approach the 'risk problem' in late modernity.

\section{Risk - values and culture}

Beck's theory of the late modern 'risk society' focuses mainly on the way risk is expressed in society at large. What he does not address to any great extent is the cultural variability in risk perspectives. These cultural perspectives of risk attend not so much to the way current social forms are reflective of risk (as in Beck's 'risk society') but to the way societal forms themselves affect the way decisions about risk are made. In a sense Beck's risk society is a top-down approach. How social forms effect risk decision-making is a more bottom-up approach. The most influential thinker in this field is the anthropologist Mary Douglas. She points to the lack of uniformity in opinions about what makes something risky, how risky it might be and what should be done about it (Douglas \& Wildavsky, 1982). She rejects both the scientific, objectivist approach and an individual rational choice approach to risk decision-making but proposes that risks are decided upon according to the cultural meaning associated with them. She is critical of experts' attempts to get to the objective truth of risk by protecting it from the 'dirty' side of politics and morals.

Placing all the focus on individual cognition excludes the problem. The risk perception analysts say practically nothing about intersubjectivity, consensus making, or social influences on decisions (Douglas, 1992, p12).

People, she proposes, do not make decisions about risk according to individualised circumstances and beliefs but are culturally conditioned to prefer some types of decisions over others. Their beliefs and actions therefore are culturally constructed. Within any culture there will be subgroups and communities who have varied value bases and ethical systems. These ethical systems too are culturally constructed and may vary. This variety is not related to any misguided perception, as objectivists would propose, but to different political, moral and aesthetic positions (Lupton, 1999).

Values and uncertainties are an integral part of these choices and Douglas proposes that the choices between risky alternatives are not value-free. Choice in the end therefore, is essentially based on social rather than scientific knowledge (Fischhoff \& 
Lichtenstein, 1981). This decision-making process can also be seen as political. Who should make decisions, who and what should matter are related to whose knowledge is regarded as authoritative. Douglas's position does acknowledge that dangers and risks are real but proposes that it is impossible to rank them in any rational sense. There are simply too many of them (Douglas \& Wildavsky, 1982). A cultural approach therefore helps us see risk decision-making as a result of community consensus, rather than rational individual choice. It is this community consensus that gives preference to some risks over others. According to this argument, dangers are selected for public concern according to the strength and direction of social criticism.

The choice of risks and the choice of how to live are taken together. Each form of social life has its own typical risk portfolio. Common values lead to common fears (and, by implication, to a common agreement not to fear other things). There is no gap between perception and reality and no correct description of the right behaviour, at least not in advance (Douglas \& Wildavsky, 1982, p8).

Attempts to avoid or control risk are problematic as there are real dangers associated with too tight and narrow a control. This, says Douglas, can lead to a false sense of security, a lack of flexibility and can compromise coping ability.

If some degree of risk is inevitable, suppressing it in one place often merely moves it to another. Shifting risks may be more dangerous than tolerating them, both because those who face new risks may be unaccustomed to them and because those who no longer face old ones may become more vulnerable when conditions change (Douglas \& Wildavsky, 1982, p197).

Heyman (1998) applies this idea to the problems health practitioners have when informing or supporting their clients risk decisions:

Difficult decisions about risks entail weighing-up and trading-off qualitatively different values, for example autonomy versus safety, or quality of life against longevity. Such trade-offs of finely balanced but incompatible ends require value judgements which individuals and social groups make differently, and which cannot be meaningfully aggregated or anticipated. The persons most directly concerned in a health decision may have difficulty in deciding or even predicting what they would do. Health 
professionals who wish to help clients to manage risk need to grasp the complexities involved in such reasoning (p9).

\section{Risk, danger and blame}

In her later work, 'Risk and Blame, Douglas (1992) addresses the idea of blame, making a connection between pre-modern and modern constructs. Her 'forensic theory of danger' explains how people apportion blame, which Douglas says applies in both pre-modern and modern cultures. Following this theory, misfortune is caused either by moral wrongdoing or is the work of an adversary. Misfortune caused by moral wrongdoing leads to conformity and conservativism. Caused by adversaries (the Other) the response is a demand for compensation, vengeance and punishment.

With the advent of science and technology Douglas had thought that 'real' sciencebased blaming as opposed to superstitious blaming could occur. Objective knowledge would tell us the truth of the matter, the assumption being that modern life had severed the link between morality and danger; we could now know risks objectively. However, technology, the dominant factor in modern life began to be seen as a source of risk itself.

Then suddenly technology itself came under attack as the source of danger. ...It became plain that the old link from danger to morals was not made by lack of knowledge. Ambiguity always lurks. If you want to cast blame, there are always loopholes for reading the evidence. ... Industrialization has not produced a race of human beings disinclined to use danger in the rhetoric that protects the public good. The difference is not in the quality of knowledge but in the kind of community that we want to make, or rather, the community we are able to make, or should say, the community that technology makes possible for us (Douglas, 1992, p9).

Danger for Douglas is therefore synonymous with risk, as risk is inherently a culturally and value laden construct. It is neither objective nor measurable. Douglas provides the example of the quantification of risk as applied to the prenatal diagnosis of Down's syndrome. The mathematical probability of having a Down's syndrome baby versus the probability of miscarrying following the diagnostic test is a good example of current professional discourse in relation to risk. Decision-making is seen 
as an objective, rational choice. She states that disputes about risk and blame have become 'endemic and self-generating'.

The doctors, knowing that they are going to be sued if they give the wrong advice, have to practise a more cautious medicine, their manners have to be formal and distant, they watch their words, and resort to objective probabilities to explain the choices that face their patients. They must not advise in these choices, as they may be sued for the wrong advice. The scientific language of risks allows them to let the patient choose for herself (Douglas, 1992, p14).

Douglas does share Beck's interest in the role globalisation has paid in our increasing attention to risk. She sees globalisation as causing society to lose its 'old protections'. The markets suck us (willingly) out of our cosy, dull, local niches and turn us into unencumbered actors, mobile in a world system, but setting us free they leave us exposed. We feel vulnerable (Douglas, 1992, p15).

At the national and international level the concept of risk therefore fits perfectly.

Its universalising terminology, its abstractness, its power of condensation, its scientficity, its connection with objective analysis, make it perfect. Above all its forensic uses fit the tool to the task of building a culture that supports a modern industrial society (Douglas, 1992, p16).

Risk in this context always looks to fault and asks

...what action? Which means, what damages? What compensation? What restitution? And the preventative action is to improve the coding of risk in the domain which has turned out to be inadequately covered. Under the banner of risk reduction, a new blaming system has replaced the former combination of moralistic condemning the victim and opportunistic condemning the victim's incompetence (Douglas, 1992, p16).

Douglas is also interested in the political implications of this and discusses the importance of the notion of blame as a mode of social control. Blaming is a way of manning the gates and at the same time of alarming the guard (Douglas, 1992. p19). 
One gets the sense from her work that she finds blame counterproductive. In her work she presents examples of cultures where forgiveness rather than blame is valued and relates this to the possibility of no fault compensation insurance schemes within modern cultures.

Although Douglas does see that technology and globalisation has had an impact on increasing the awareness and anxiety related to risk, her interpretation of the origins of blame is different from Beck's, and reflects her position as an anthropologist. For Douglas, modern constructs of blame originate in the symbolic nature of purity and pollution, which she sees as the source of anxiety and fear (Douglas, 1992). Blame for Douglas is the modern equivalent to the rituals associated with impurity and danger. Is it deflected onto the 'Other', either an individual or a group. For Beck however, current constructions of blame are a response to the reflexive nature of the late modern society reflecting the anxiety related to loss of control and trust in modernity itself. He is less inclined to stress the importance of collective priorities. However Beck's blame is also focused on the 'other' but in particular to those in power, the experts and the decision-makers.

For Douglas blame and liability are connected to pollution and impurity through the concept of normality.

Blameworthiness takes over at the point where the line of normality is drawn. Each culture rests upon its own ideas of what ought to be normal or natural. If a death is held to be normal, no one is blamed. Debates about new technology put into question the old perceptions of the natural and normal. The new technology produces new social responsibilities and provokes cultural reassessment. The line around normal dangers has to be revised to sharpen responsible behaviour by refocusing blame (Douglas \& Wildavsky, 1982, p35).

Pollution and impurity implies an abnormal intrusion of an alien element. This carries with it political and moral implications. It is fundamentally important therefore that what is considered normal has moral and political overtones. Changing cultural perceptions of what is considered normal and the challenges that technology poses to 
this are fundamental difficulties to the extent that technology itself can be considered either an abnormal intrusion (a pollutant) or a life-enhancing medium.

\section{Summary of Douglas's view of risk}

Douglas's view on risk shares some of Beck's interests yet adds a perspective that is important in informing this research. Douglas's views add to the understanding of the role of what is considered normal. Her perspective on blame and the implications this has on how experts relate to individuals are important to consider when viewing the way midwifery care is provided. Above all she provides the perspective that risk decision-making is culturally determined and value-laden. This, she proposes plays a more important role in decision-making than scientific estimations of probability. Thus a study describing and interpreting how midwives make sense of risk in practice needs to be attentive to this. Interestingly just as Beck has called for a paradigm shift in the way risk is understood and managed so too does Douglas. She critiques the way blame is managed and almost plaintively wishes for the possibility of forgiveness. This study is attentive to the effects of the 'name, blame and shame' culture and investigates the extent to which midwives manage to incorporate it into their work.

Douglas also addresses the political nature of risk decisions. Power and authority are important considerations. This perspective is the focus of those who tackle the 'risk problem with a post-modern lens, particularly those who take their lead from the work of Foucault.

\section{Governmentality and surveillance}

Those who write about risk from the Foucaudian perspective reject risk as being 'real' in any sense. It is instead seen as a construction, an exercise in power and control. Central to this approach are the ideas of governmentality and surveillance. Governmentality, the way political power has been represented during modern times, focuses on the rights of the individual as opposed to the rights of the state. It is the expert knowers rather than political rulers who decide how people should behave. This power is exercised by collecting information about what is 'normal' (surveillance) and then distributing this knowledge in a way that exhorts the population to conform. Risk is constructed as a way to control or discipline the masses. Power then is expressed overtly in regulation and, more importantly, covertly 
in relying on the individual internalising the information and self-regulating. A failure to care for the self is regarded as a moral failure and risk avoidance as a moral imperative (Lupton, 1999).

Foucault's notion of power is one of his most important contributions. He challenged the concept of power as 'macro-structure' proposing that power is a relationship which was localised, dispersed, diffused and typically disguised through the social system, operating at a micro, local and covert level through sets of specific practices (Turner, 1997). He also saw power as being inextricably tied to knowledge. The growth of knowledge is always associated with an increase in surveillance and discipline related to that knowledge.

Castel (1991) makes an important comment on how this process has fundamentally changed the interactions between medicine and patients. The growth of surveillance, he says has seen the gradual erosion of the personalised nature of the consultation. The examination of the patient has now become more of a collation of a dossier rather than a personalised assessment. This dissolves the notion of a subject or a concrete individual, and puts in its place a combination of factors, the factors of risk (Castel, 1991). Thus the caring function is transformed into an activity of expertise, leading to its subordination to administrators. Further, this approach to surveillance has changed the notion of an individual being dangerous, either to themselves or others, to being 'at risk'. This says Castel leads to an enormous growth in the quantity of risk factors and is, as in both Beck's and Douglas's perspective related to control.

The modern ideologies of prevention are overarched by grandiose technocratic rationalizing dreams of absolute control of the accidental, understood as the irruption of the unpredictable. In the name of this myth of absolute eradication of risk, they construct a mass of new risks which constitute so many new targets for preventative intervention (Castel, 1991, $\mathrm{p} 289)$.

This sounds remarkably like modern obstetrics! Risk then is an important tool. This approach, like Douglas's, relates risk to the idea of what is and is not normal. Normalisation is valued. It also implies that population data are used in advice given to individuals (the ecological fallacy). It can incorporate the ideas of Beck's risk 
society. A global deregulated society needs to have more effective micro-surveillance and discipline, more subtle forms of control (Turner, 1997).

\section{Challenging risk: a case for midwifery}

These perspectives all add to our understanding of current constructions of risk. They point to the dominant role that risk plays at this point in modern, or 'late modern' life. There are three concepts related to risk that run through these perspectives: normality, control, and blame. Being normal is important, both in an epidemiological and cultural context. Being outside normal or deviant can thus be seen as 'risky' and dangerous. In maternity care control is manifest in technology and in the dominant discourse of medicine. The experience of birth is defined in terms of differing levels of risk. Technology promises to eliminate the risks of birth. Thus the medicalisation of birth promises a risk-free birth and allows obstetricians to decide which births can be safely handled by midwives (De Vries \& Barroso, 2000). Control of deviance (managing risk) can thus be seen as important. Control in maternity care is manifest in multiple ways: institutionalisation of childbirth in hospitals, regulation of health professionals, subservience of midwifery to obstetrics, and the use of protocols and guidelines. Blame is a powerful tool for managing or controlling deviance. Blame, in late modern societies turns in on itself. It is a quality of its reflexivity. When technology fails, when risk is expressed in poor outcome, someone must be held accountable. Loss of faith in technology and therefore in expertise translates as loss of trust in professionals with the corresponding growth in anxiety related to litigation (Symon, 1998). Thus medicine itself, in anthropological terms, becomes the Other. Its lack of ability to fulfil the promises of technology makes it the deviant one. This reflexivity is also manifest in the confusion related to normality. What used to be considered as normal is both challenged and usurped in a paradoxical fashion. In a sense it has become normal to be 'at risk'. Thus societal forms become confused and contradictory.

How then has midwifery positioned itself within these contradictory forms? All these theoretical approaches speak to midwifery. Midwifery can be seen as an agent of surveillance influencing the woman to conform, to become normal. It can also be seen as an agent of expertise, challenged by the risk actors, the consumers of 
maternity care. All these approaches have talked about medicine. What about midwifery? Is it an extension of medicine or of the consumers? The increasing tendency to define all births in terms of 'risk' (low or high) has a profound impact on the profession of midwifery, allowing the definers of risk to determine the work of midwives and to create an image of midwives in the minds of the public. Does midwifery construct risk this way? Does it position itself alongside medicine or alongside the consumer? Has risk overtaken the relationship? These are some of the issues that are addressed in this research. When we place partnership as central to midwifery, and the woman rather than the foetus at the centre of care, are we trying to recreate a way of being a midwife that is now obsolete? If we are managing womancentred, partnered care what does it look like and where does risk fit? The risk environment I propose is our principle challenge.

Looking at risk in maternity care from a variety of theoretical perspectives poses many questions and certainly would benefit from empirical evidence to support or challenge the assertions that are made. The theoretical approaches discussed above both begin to put risk in midwifery practice into a context and to open up more questions. The main issues which are addressed include: do New Zealand midwives feel part of Beck's 'risk trap' and how is this expressed in practice? To what extent do we incorporate social and cultural understandings of risk into our practice? How do we place the consumer in risk decisions? How do we place our practice within the birth is normal/birth is risky dichotomy? Do we believe that midwifery should concern itself only with normal birth and what is normal birth?

New Zealand is a fascinating place to examine this. We are at the forefront of an international resurgence of midwifery and we have made significant inroads into progressing the place of the midwife. We have autonomous state funded midwifery care, equal pay with doctors, direct entry midwifery education at undergraduate level, and growing postgraduate education, as well as representation at every level of local and national decision making in health care. The majority of New Zealand women now choose a midwife as their lead maternity caregiver. There are concerns however about how we are progressing. There seems to have been minimal rise in the home birth rate, and our intervention rates have continued to rise (Standards New Zealand, 2001). This may be a manifestation of the 'risk trap' in which midwifery is still 
required to function or it may be that we have replaced medicine, consciously or unconsciously in maternity care, usurping its role but not changing it. It is certainly worth exploring.

\section{The research - a place for the theory}

The theoretical approaches presented assist in informing both the research question and the research design. The work of both Beck and Douglas call for research on risk which crosses epistemological divides.

Risk science without the sociological imagination of constructed and contested risk is blind. Risk science that is not informed about the technologically manufactured 'second nature' of threats is naïve. The ontology of risk as such does not grant privilege to any specific form of knowledge. It forces everyone to combine different and often divergent rationality-claims, to act and react in the face of contradictory certainties (Beck, 1999, p4).

Douglas also proposes this approach suggesting that insights from social anthropology, sociology, politics and culture should be incorporated into the study of risk and in the discussion of responsibility. She says:

A more holistic approach to the subject of risk is required, one which is not compartmentalised. It should be across nations, cultures and across disciplines. Risk should be seen as a joint product of knowledge about the future and as requiring consent about the most desired prospects. This may enable us to put the problems into perspective (Douglas, 1992, p57).

Heyman (1998) contends that research on risk needs to have a variety of approaches. He proposes that a ...serious analysis of risk requires the social scientist to straddle uncomfortably the academic cultural divide between art and science, and between qualitative and quantitative paradigms. Risk analysis involves both engagement with the numerical complexities of probability, and consideration of philosophical questions, such as what makes a probability probable or an adverse event adverse (Heyman, 1998, p14). 
Heyman further contends that neither the objectivist approach nor the subjective approach alone can connect the reality of the physical risks with human perception of them. Wilkinson (2001) too comments on this need for multiple views into the issue

While the 'risk debate' may be conceived in part as a direct result of the greater sophistication and social application of our techniques of calculative reason, we should recognise it is comprised of moral judgements and political values which defy analysis in these terms. ...However, ultimately it may be impossible to distinguish differences of opinion at one level of discourse as entirely separable from the dynamics of the other (Wilkinson, 2001, p100).

Lupton (1999) provides a useful summary of the epistemological sites of these three theoretical approaches to risk. The techno rational perspectives on risk, she describes as 'realist', interested in what risks actually exist and how they might be managed. The sociocultural approaches she describes as 'weak constructionist'. These approaches ask how risk is related to the social and cultural context in which it is situated. The 'strong constructionist' approach proposes that risk is itself a social construction rather than real in any sense and is interested in the discourse of risk in relation to subjectivity and social life. These three positions present theoretical positions from different epistemological locations yet all seem to have relevance for midwifery and how we practice. The question asked in this research concerns how midwives make sense of risk in their practice. It is a broad question, opening up an area of inquiry not extensively explored. The following chapter explores how risk might be investigated from a midwifery perspective taking into account this variety of epistemological positions. We need to do this in practice. It therefore would seem a worthwhile endeavour to attempt to undertake this research reflecting what happens in practice.

The research therefore takes an epistemologically pluralist position. Each of the perspectives offers something to the research question. The techno rational approach to birth asserts that risks are real and measurable. This approach is important, as risks for the midwife are real. Although the sociocultural approaches, apart from the strong constructionist approach, do acknowledge that risks are real, their position is not strong enough on its own to sustain what I think is the real work of providing 
maternity care. This approach has added, and continues to add, important information to enhance the way we provide care (Oakley, 2000). Sociocultural approaches also underemphasize the affective or relational work of medicine as reflected in current discourses of medical care. This polarisation of knowledge, between quantitative and qualitative paradigms, is unhelpful in the quest to understand the role of the midwife in the real and complex world of risk. For the midwife both these approaches are real; risk is real. One is not more important than the other. These theoretical positions support a research approach that takes an epistemologically pluralist position. This position is also appropriate given the complex and multidimensional nature of midwifery's risk work. In order to understand how midwives deal with risk in practice, neither the subjective not the objective nature of risk can be discounted. Being alongside Bronwen and Mele as they prepared to give birth and made their risk decisions was a complex process involving science, technology, culture, and society. None could be discounted. As a work of midwifery therefore this research needs to investigate how midwives deal with risks and with risk. It needs to take into account the whole of midwifery practice.

\section{Operationalising risk}

These theoretical approaches also support the referral for obstetric consultation as the place where risk in practice could be operationalised. The referral is the place where risk is identified. This is where midwifery comes up against medicine reflecting different ways of knowing and different understandings of what is normal. It is in this place that value systems and positions become evident. Power and control over decision-making are also overt. Responsibility, accountability and blame are visible here, as when complications arise, obstetrics is usually both involved and authoritative. At this point surveillance is at its most concentrated. Action in this moment is governed and explicit. It is also the site of the three-way interaction between doctor, midwife and childbearing woman. Here the midwife becomes both subject and object of surveillance and control. It is therefore the ideal place in which to investigate how she makes sense of her role in relation to risk.

Midwifery is in a perfect position to participate in and develop both the theoretical and practical ramifications of research that takes a pluralist approach. We have a history that was, until recent times, isolated from a front line place in the development 
of modernity's mind/body separation. Current constructs of midwifery point the way to an understanding of birth that is different (Sandall, 2000; Skinner, 2002). Individuals are increasingly asking for a say in their birth experience, they want choice and control. They want a human, not a mechanistic birth (Bourgeault, Declercq, \& Sandall, 2001; Thomas, 2002). Midwifery in New Zealand has responded to this by developing partnership as its philosophical base. On an international front, the resurgence of midwifery points to a direction that runs counter to the traditional risk approaches and reflects a different way of viewing birth; a more holistic, inclusive, permissive and expansive model (Lay, 2000). It is hoped that midwifery will humanise birth, view women as not only physical, but also as social, cultural and spiritual beings. The growing movement for consumer participation and rights, the trend towards evidence based practice, the wide variety of choice that women have about where and how to give birth, all point to a new way of being in the world and of dealing with risk and its consequences.

\section{Summary}

The theoretical perspectives presented in this chapter provide a framework in which to understand midwifery and its relationship with risk. It is a background in which the techno-rational project of modernity has dominated. The quantification of probability has had a profound impact on the way maternity care is provided. This has been weakened in more recent times by global anxieties, which Beck describes as 'risk society'. This reflexive late modernity engenders anxiety about life. Almost everything is seen as risky. We are losing faith in the modern project yet we cling to it still in the hope that all risk can be managed, that we can still colonise our future, that technology will save us. On the one hand we have an understanding of risk as being part of being alive, on the other hand we have the transformation of all risk as being avoidable. We have lost faith in the ability of professionals to know all, yet expect that with better risk management professionals will produce the perfect result. Within this context we also understand that risk perception and action are culturally mediated. Risk and its management are heavily value and culturally laden.

The increase in the emphasis on risk has resulted in an increase in managerialism and consumerism. The individual is seen as less willing to accept a negative outcome; is 
in a sense not accepting of risk, denying its reality. Blame has led to increased accountability and has resulted in the institution's attempts to increase surveillance and control. Risk management has become risk aversion.

Within this context we can examine midwifery practice in more depth. Bronwen and Mele's risk stories start becoming more comprehensible. What is evident is that any attempt to understand midwifery practice in the context of risk must take all these perspectives into account, as all have an impact on the way midwifery takes place. Some attempt must be made to investigate midwifery across the spectrum of epistemological positions. The following chapter provides a philosophical framework and methodological perspective that support how this rather complex and onerous task might be undertaken. 


\section{Chapter 3: Methodology}

\section{Introduction}

The previous chapter has highlighted the multiple theoretical perspectives from which one might view risk, from the techno-rational to the post-modern, from epidemiology to sociology and anthropology. It has identified the strong cultural and social impact on how risk might be perceived and it is these approaches, particularly those of Beck and Douglas that form the theoretical backdrop for this research. My main interest is in how midwives make sense of practice within this societal and cultural milieu and how this might be expressed in action and thought - what they do and how they think. This chapter discusses the nature of midwifery and proposes a way that the expression of risk in midwifery may be investigated. It reintroduces and focuses the research question and identifies the referral for obstetric consultation as a place where risk in practice might be best identified. In order to clarify the ontological position of midwifery and of the research it draws on the philosophical approach of critical realism. This perspective has a number of aspects in common that seem able to support the nature of midwifery (its ontology), the way midwives know the world (its epistemology), and that therefore support the way midwives might seek to know more (a research approach). Critical realism therefore informs the philosophical approach of the research and supports the way the research has been designed and interpreted. This approach also supports the complex theory associated with risk, in all its manifestations.

The focus of this chapter is to provide a philosophical nest for the research. Critical realism is described and applied to the research question and the chosen research approach. The overall research design is then provided. This consists of a national survey of midwives' referral for obstetric consultation practices and attitudes, and a series of focus groups held with midwives throughout New Zealand. The chapter then concludes by introducing the ethical issues related to this research. 


\section{Where to stand: A place to be and a way to know}

Risk is a complex and multifaceted concept with no concrete form. When the event that was 'at risk' of happening actually occurs, then risk disappears. It is nebulous and ephemeral, yet strangely real. It has such impact on the way humans think and act. For the midwife risk is pivotal to the way we work. We work with women in the process of childbirth. We are there to support and encourage. It is an interesting place to be, alongside a process that usually doesn't need us to do anything. We are there in a sense because of risk. Risk is our job. Women from time immemorial have called on midwives to be with them and to help should there be difficulties. Contemporary midwives are now prepared in tertiary educational institutions. We are taught how to be and what to know. Our education prepares us to be the experts in normal birth, not only to be companions, but also so that we can identify risk, manage the complications and seek help. On one level it would seem such a simple task. The various theoretical perspectives on risk though would belie this. Bronwen and Mele's stories also reveal how challenging and complex it can be. This research seeks to help in the understanding of how midwives deal with it, how they manage it and how they make sense of it.

\section{Risk and midwifery knowledge}

It seems as if all the theoretical approaches to risk could apply in some sense to the working world of the midwife; we need to understand the physiology, the epidemiology, the psychology, the anthropology and the sociology of risk. It all affects us. It is part of what we need to know. Yet this work is not the work of a scientist, or an epidemiologist, or of a sociologist or anthropologist. It is the work of a midwife. What does a piece of research by a midwife look like and what is the basis of midwifery knowledge that a midwife might draw on in order to make this the work of a midwife, rather than that of a sociologist or an epidemiologist? Does it need to worry about this? What is the ontological and epistemological position of midwifery? Does it have one, or does it need one? In a sense this chapter is a thought experiment. It looks at refiguring the risk world of the midwife and placing it within the context of 'grand theory' in order to understand how we manage it and what then might be the best way to proceed. 
There are further questions that are posed. Is midwifery an academic field in its own right or does it need to be? Does it need to have its own body of knowledge or does it even have one? When writers talk about fields or disciplines they refer to such disciplines as history, philosophy, psychology, sociology and medicine, and more recently such disciplines as women's studies, criminology, environmental studies. Where does midwifery fit? What contribution to knowledge has midwifery made? Historically midwifery has not developed as an academic discipline. It has been a craft, seen as women's business. Its knowledge has been expressed and shared practically and intuitively. The shift in midwifery knowledge from local women's wisdom to postgraduate university education has been a long story, not possible to detail here. It has been a story dominated in large part by males and by medicine and also by nursing, but supported by women in a context of the development of modernity.

I have felt some insecurity about the quality and quantity of midwifery knowledge that we can call our own. Is midwifery undeveloped intellectually? It seems that the knowledge we draw on in developing and being a midwife is drawn from other professions. The list is exhaustive: epidemiology, sociology, psychology, physiology, mathematics, history, medicine, politics, anthropology, demography, biology, genetics, women's studies and much more. Perhaps we can call ourselves a transdisciplinary profession. The concern with this position in the current context is that it lacks an intellectual grounding to give midwifery strength and status in a world where knowledge is power and it risks the possibility that we become lost in others' interpretations of the world. The advantage of this position however is that we are not restricted by any particular body of knowledge. Our wondering is free to explore multiple ways of knowing, something we are very good at. What we do 'know' however, is how to 'be' with women in birth, more of an ontology than an epistemology.

\section{Ontology and the midwife}

The notion of ontology is particularly important for the midwife because, essentially that is what we enact. We 'be' with women, alongside them as they give birth. Essentially we are not there to 'do'. Our knowing comes from our being. That is why ontology in this work is basic, primary and explicit and why I spend so much time 
exploring how we place ourselves. We are the specialists at being and of seeing how differently others 'are' in their own worlds. This research question originates in my own dilemma of how to be with women 'at risk' and seeks to understand how others do it. It does this in the context of the reality of birth and acknowledges multiple ways of understanding.

The midwife works in both the real world and the experiential world. She holds newborn babies in her hands, she feels the dilatation of the cervix, she sees the eclamptic fit, she prevents the postpartum haemorrhage, she cries, she laughs, she worries. She also works in the symbolic or cultural worlds, her own and that of the others she works with and alongside. She also works in the conceptual world. Risk itself is a constructed concept. What this piece of research aims to do is to ask questions about the relationship between the real world and the conceptual world in the context of risk. It is an experiment in midwifery knowledge.

\section{A place to settle}

In order to explore the notion of risk in midwifery a place must be chosen to study; a place where risk is best expressed. I have identified the referral for obstetric consultation as one such place where the midwife sees and acknowledges risk in practice, either risks to the mother or baby, or risk to herself. Both these aspects of risk are interrelated, as risk to the midwife is inevitably the result of an incident where risk may be expressed in the outcome for the mother or the baby. Decisions about risk management are inevitably connected at this point. This is a pivotal place, the ontological crisis point where a midwife's scope of practice is faced, defined and articulated; where the medical model meets the midwifery model; where health meets ill health; where low risk meets high risk; where men's ways meet women's ways; where normal meets abnormal; where doing meets being; where quantity meets quality; where doctors meet midwives; where yin meets yang; where modern meets post-modern; where the community meets the institution; where birth meets death; and where love meets fear. It is the place where we face the nature of multiple ways of being and knowing. It is in fact not simply a midwifery matter but may be used as an exemplar of the place where world society is at the moment. It is a global issue not just for midwives, but for us all, and it sources knowledge from many different disciplines. 
I come to this work therefore as a knowledge synthesiser, based in a way of being immersed in the journey that we take alongside women during the sacred process of birth, a journey that has become increasingly defined by risk. This study explores how contemporary midwives deal with risk. To do this it places risk within its current socio-cultural context and asks how midwives perceive and use their knowledge and their power. I use Beck's 'risk society' and Douglas's ideas about danger and the techno rational approach as theoretical informants for the research. To a large extent they remain in the background as the research is undertaken. They are reintroduced and critiqued towards the end of this work as data is analysed and a new understanding of how midwives deal with risk is developed.

This is not the work of a sociologist. It is the work of a midwife. This difference is manifest in the way I have chosen to operationalise the research in order to connect it to real midwifery practice. The advantage of coming from this midwifery position is that I can be free, to some extent, from conventional social science. It is here where being a midwife is most useful. We have no particular 'ology' to protect and are free to use whatever approaches might allow us to be creative in exploring this issue. I am able therefore to keep connected to the realities of practice and to the lives of midwives. After all, this is the purpose of the research and the purpose of my work now; to help midwives in practice so that they might better care for women.

\section{Critical realism: Help from a philosopher}

I discovered the philosophical approach of critical realism in the process of undertaking this research. It has been helpful in many ways. It has spoken to the multi-layered and complex nature of reality and thus was for me a possible philosophical position from which to examine the complex world of the midwife and the concept of risk. It has been particularly influential in informing the way the data for this research has been gathered and analysed. It is beyond the scope of this research to provide an in-depth discussion of the complex and dense tenants of critical realism. However, what is provided is a summary of its basic propositions and a description of how it has informed this project. In summarising the ideas associated with critical realism and in providing the evidence of how it relates to this research project I have drawn heavily from the work of Berth Danermark, Mats Ekestrom, 
Liselotte Jakobsen and Jan Karksson in their book Explaining Society (1997). This book provides guidance as to how the philosophical position of critical realism can be used in the research process.

Critical realism emerged during the 1970s and its central figure is the English philosopher Roy Bhasker. Bhaskar (1986) deals with the difficulty faced by contemporary philosophers of science in dealing with the demise of positivism yet, in his opinion being unable to sustain the arguments proposed by relativism. $\mathrm{He}$ discusses the approaches of such thinkers as Bachelard, Popper, Kuhn, Hume, Hegel, Kant to name but a few, and develops an ontological approach to philosophy which may in his view provide a way out of what he considers a 'prison of paradox'. His key works include: A realist theory of science (1978) Scientific realism and human emancipation (1986), Philosophy and the idea of freedom (1991), and Dialectic. The pulse of freedom (1993). He has been identified as one of the most important contemporary Marxist philosophers. His primary interest is in the philosophy of science but he covers an enormous body of knowledge including the theory of knowledge and causality, ethics, politics and the sociology of philosophy (Spencer, 2002). He continues to develop his philosophical understandings and his latest book From East to West: Odyssey of a Soul published in 2000 has taken a decidedly spiritual turn, startling many of his followers. It deals with the existence of God and the need for unconditional love! Other key critical realist writers are Margaret Archer (1998; 1999; 1995), Andrew Collier (1994)and Andrew Sayer (1992; 2000).

\section{The nature of reality}

Critical realism, as the name indicates proposes that there is such a thing as reality. Bhaskar's realist position asserts that this reality exists at three different levels. He calls these levels the 'real', the 'actual' and the 'empirical' (Archer, Sharp, Jones and Woodiwiss, 1999). The 'real domain' is the deepest domain and is where the generative mechanisms exist, that is, where power and action originate. This domain is not directly observable and it is this dimension that science, both natural science and social science, attempts to investigate. It is this deep domain that distinguishes critical realism from other forms of realism (Danermark, Ekstrom, Jakobsen, \& Karlsson, 1997). Bhaskar's approach has also been named 'transcendental' critical realism and also 'depth' realism exactly because of this focus beneath the actual 
events in order to discover the mechanisms that generate them (Collier, 1994). Bhaskar proposes that when these mechanisms in the deep or 'real' domain are activated they cause events, which occur in what he calls the 'actual' domain. These events in the actual domain may or may not be experienced; an apple falls whether you see it or not. The next level up he called the 'empirical domain'. This is the level of experience where events, facts, occurrences are observable. In research terms this 'empirical domain' is where we find our data. This data is always mediated by our theoretical conceptions of it. The work of science therefore is to "...investigate and identify relationships and non-relationships, respectively, between what we experience, what actually happens and the underlying mechanisms that produce events in the world" (Danermark et al., 1997, p2).

Bhaskar also describes reality as being 'stratified'. For example, there is a chemical, a biological and a social stratum, and each of these strata has their own generative powers. As you move up the levels the strata become more and more complex as the generative powers interact. It is possible to examine the lower level strata as closed systems because the generative powers within this level can be controlled (the positivist approach). In the social stratum, the most complex level, it is not possible to create a closed, controlled system. The generative powers at this level, whether dormant or active, cannot be isolated. Events at this level are caused by a variety of mechanisms and in most situations there are many things that determine what will happen, making it difficult to see what is causal (Spencer, 2002). This has significant implications both for the way science can be undertaken and how close we can get to understanding causation.

\section{Cause}

Critical realism is fundamentally interested in causation. Events do not happen without cause. "Critical realism consequently takes its entry point on the basic assumption that objects in reality possess causal powers, that is, generative mechanisms" (Danermark et al., 1997, p198).

It proposes that behind events are powers or 'mechanisms' that have caused them. It is the task of science, whether natural or social, to discover these causal or generative powers (Bhaskar, 1978). Much of social science research has not gone beneath the 
empirical level in its investigation of cause and has often been mistaken in its conclusions; correlation for example does not prove cause. Critical realism proposes that social science must look deeper into the 'real' domain in order to begin to establish cause in any useful sense. In realist terms, causes are about objects or their relations and their nature. It is a matter of how objects work, a matter of their mechanisms. Causal powers can be located in the natural world but can also be located in the social relationships and social structures that people build (Danermark et al., 1997). Cause then is not a matter of simple cause and effect.

Often the causal powers inhere not simply in single objects or individuals but in the social relations and structures which they form. ... On this view then, a causal claim is not about a regularity between separate things or events but about what an object is like and what it can do and only derivatively what it will do in any particular situation (Sayer, 1992, p105).

These causal powers exist whether they are exercised or not. Water can put out a fire irrespective of whether anyone uses it to do this. People are capable of working even when they are unemployed, and are able to love whether or not they ever enter into a love relationship. On the other hand, the existence of causal powers is not an assumption about fixed, unchangeable, eternal essences (Danermark et al., 1997). Cause then in the social world cannot be discovered in any simple, fixed 'cause and effect' sense. Explanation can be proposed but will always be tentative and open to change and challenge. It many ways it is remarkably similar to risk.

Because critical realism proposes that there is a reality, independent of our conceptions of it, it proposes that reality can and should be examined in order to grow knowledge. Science, the process of examining reality, has two dimensions. The external dimension, the independent reality, Bhaskar calls the intransitive object of science. We come to know and understand this intransitive object by developing concepts and theories about it. These concepts and theories Bhaskar calls the transitive dimension of science. They are the raw material of science connecting it with reality (Collier, 1994).

Theories then are the best truths we have about reality at any given moment.

The 'results' of scientific enquiry at any given time are a set of theories about the nature of the world, which are presumably our best 
approximation to truth about the world. But the work of science at any time takes these theories as its raw material and seeks to transform them into deeper knowledge of the world. These theories are its transitive object; that it seeks to transform them shows that its aim is knowledge of its intransitive object, the world that exists independently of it (Collier, 1994. p51).

This has implications for the nature of knowledge and for how we might go about developing it.

\section{The nature of knowledge}

Knowledge is not spontaneously given to us but must be discovered through investigation and interrogation, particularly within the transitive realm, the realm of concepts and theory (Archer et al., 1999). All knowledge is mediated through the use of concepts and it is thus impossible to make 'neutral' observations about reality.
All knowledge is necessarily socially determined conceptual constructions. Facts - the empirical observations, scientific data - are seldom objective or neutral in any definite sense. To be at all understandable they always comprise earlier, more or less hidden, everyday and/or scientific conceptualisations. That is, facts are theory- dependent or theory-laden (Danermark et al., 1997, p17).

Critical realism refutes the possibility of objective or 'true' knowledge. What we know is not necessarily the truth. It is the purpose of science to get as 'close as possible' to the nature of reality.

\section{Concepts and theories}

Abstraction and causation are core functions of scientific enquiry. In order to be able to abstract we must acknowledge the importance of concept and theory development. Concepts are the building blocks of knowledge and are the core elements of how we come to understand the world. Conceptualisation is a primary scientific activity (Danermark et al., 1997). The production of new knowledge then, relies on the development of new concepts. 
Every scientific (and everyday) attempt to understand and explain the world starts from our concepts of it. In every research project one important aspect of its aim is to influence - revise, modify, improve or revolutionize - our present concepts of reality (Danermark et al., 1997, p15).

Concepts then, are the building blocks of theories which in turn represent what we have learned about the world.

The critical role and function of concepts regarding social worlds together with the actions they inform - these can be said to mediate the existence of these worlds - are however, the reason why everyday concepts must be the very starting point, when we build the scientific systems of concepts, that is to say theories. The content of everyday knowledge is part of the 'raw material' that scientific knowledge must systematically include, if theories are to be valid. It is also one of the reasons why a contemplated analysis of concepts and concept formation is on the whole an essential activity in social science. Concepts are the very key to knowledge about society (Collier, 1994, p35).

Critical realism proposes that reality has an objective existence and that we know it through the concepts we have of it and the theories we construct about it. All facts are theory-dependent but not theory-determined. Inevitably then all knowledge is fallible, some more so than others (Danermark et al., 1997).

This means that there are no pure data; all data are theory-laden. Objective data are not really that objective; they are dependant on how we perceive them. For example, light can be perceived as either a wave or a particle since light exhibits the characteristics of wave and particle under different circumstances. This implies that our perception determines the nature of light. In other words, our perception does influence our knowledge of this external physical world. The social world is even more complex. It is always shifting and moving. Society is changeable in the way that nature is not. This makes conceptualisation within the social world very important. 
Knowing that our concepts and theories especially in regard to the social world are at any time tentative and that even establishing theories of causation is difficult, critical realism proposes that prediction is not possible. Bhaskar (1989) states

It is clearly in the human sciences that the propaedeutic work of philosophy is likely to be most rewarding - if only by allowing a better contrast to be drawn between the conditions and possibilities of the natural and social sciences. Thus the non-availability of spontaneously occurring and the impossibility of experimentally establishing closed systems means that criteria for the rational assessment and development of theories in the human sciences cannot be predictive and so must be exclusively explanatory (p24).

\section{The positivist/relativist debate}

Some further discussion is required about the positivist/relativist debate as this problem has been a primary concern in the development of Bhaskar's philosophical position. The claim that all facts are theory dependent or theory-laden rang the death knell for positivism in its pure sense. The contradictory position, that all knowledge is absolutely relative and that any search for general knowledge is meaningless is, according to Bhaskar, 'auto-destructive'. This dualism, that Bhaskar seeks to overcome had created major contrasts between positivism and hermeneutics, between quantitative and qualitative approaches (Danermark et al., 1997).

Bhaskar argues against this dualism, which he says has divided the human sciences. He argues against the dualisms of positivism and hermeneutics, collectivism and individualism, structure and agency, reason and cause, mind and body, fact and value and challenged the empiricist/anti empiricist divide. He did this by giving reality a new content, his three layered reality. In doing so he argued against the reduction of knowledge into being. Being is an irreducible and necessary category on its own. His analysis of ontology provided a 'third way' mediating between objectivism and relativism.

In each case, critical naturalism argued for a third sublating position which could reconcile these stark polarities and oppositions, and which could situate the two extremes as special cases of the more general sublating position. In dialectical critical realism dualities are punctuated 
by a hiatus, which stops a reductionist collapse and signifies a difference in orientation or spatio-temporal lag (Bhaskar, 1993, p397).

Critical realism therefore provides a philosophical position that provides an answer to cross the theoretical/empirical divide and also to solve the problem between naïve objectivism and its opposite - relativism by proposing a new approach to the nature of reality (Danermark et al., 1997). Bhaskar (1989) states that the

...realist account of science provides a clear and consistent alternative to positivism which allows us both to save the cumulative character of science without restoring a monism and to rescue a 'surplus' component in scientific theory without plunging into subjectivism ( p15).

\section{Critical realism and the dialectic}

Bhaskar speaks strongly about 'the hiatus that punctuates duality'. His work proposes that in examining this dialectic we can examine reality. For the critical realist there is no such thing as truth yet this reality does exist, independent of whether we know about it or not. Our attempts to understand this reality are important. We may seek a better understanding by taking a dialectic stance, engaging in and examining multiple and often contradictory perspectives. In order to better understand the variety and contextually of social life the dialectic response is to "... intentionally engage multiple perspectives, diverse ways of knowing and understanding, and varied ways of studying and representing human behaviour” (Greene \& Caracelli, 2003, p93).

All perspectives have something to offer. They can lead us closer to the truth, however intangible and illusive that may be. Critical realism is dialectic itself in that it engages directly in the tension created between positivism and relativism. The hiatus or dialect that I examine in this research occurs around the referral for consultation. As I have stated earlier this moment has a multiplicity of tensions. It is at the edge of midwifery practice, where it comes directly into contact with medical practice and is also a place where midwifery examines its own place in the world.

\section{The double hermeneutic}

Bhaskar's philosophical approach deals with science in general but he does make a distinction between natural and social science. In natural science the researcher's 
relationship with the object of study is a simpler subject/object relationship. This has been called a 'single hermeneutic' (Sayer, 1992). Interpretation is one sided. This relationship is very different when the object of study is the social world. This entails a 'double hermeneutic' as the social scientist's task is to interpret other people's interpretations (Danermark et al., 1997). This makes investigation of the social world more complex than investigation of the natural world. The social world does not exist as an objective entity so one cannot attain a 'correct objective' picture of it, as the more positivist approach would propose. We cannot step out of our conceptual worlds to see 'what really is' (Danermark et al., 1997).

Critical realism also rejects the relativist position which would propose that it is meaningless to make any claim about one statement about the social world being any more true than another (Danermark et al., 1997). The critical realist position is that there is a possibility of understanding the social world as we do the natural world and that those who say you cannot, have misinterpreted the reality of the natural world (Spencer, 2002). For the critical realist the reality that exists in the social world is always shifting and changing and although it is more difficult to get close to, attempts to do so are important as we strive to improve human existence.

\section{Emancipation}

This emancipatory element of critical realism is another key aspect of its approach. Critical realist research seeks to understand the world in order to improve it. This has been a consistent element through all of Bhaskar's work. He states

On analysis, ontological, epistemological, relational and critical differences emerge between the natural and the human sciences. Examination of the critical difference allows us to illustrate the passage from fact to value, theory to practice. Associated with this is a definition of emancipation and a richer conception of human freedom that the existing traditions have been able to sustain (Bhaskar, 1991, p142).

He believes that description and interpretation lead to emancipation.

It is the use of the word 'critical' that leads us to suspect the emancipatory nature of critical realism. As we attempt to understand social life and consciousness, and as we 
expose the generative mechanism, that which is normally unexamined, ... we cannot help but become aware of its illusions (Sayer, 1992). Critical realism aims to look deeper into everyday thinking. Such thinking may be rational and understandable yet may be caught up in myth, illusion and ignorance. When this is revealed and challenged opportunities are created for critical thinking and for change (Danermark et al., 1997). Critical realism is also self-critical. It recognises the limited possibilities of science itself and is critical of any universalist claims to truth. It also recognizes the personal role of the scientist in theory generation. The scientist herself comes to research with her own social understanding (Archer et al., 1999). As all knowledge is fallible, so the account that the researcher provides is, and should be, open to critique and reinterpretation.

When we lay bare the generative mechanisms at the social level, we thereby also explain social phenomena in terms of social causes. These are produced by people and can thus be changed by people. Relations of dominance and power can easily be seen (Danermark et al., 1997, p201).

\section{Fallibility}

Throughout critical realism therefore, there is an underlying understanding that all knowledge we have of the world is fallible and incomplete. The world is perceived conceptually, and these concepts are theory-laden. They are filtered through our own and others' experiences. We cannot move outside of these concepts to perceive what really is. So we can make mistakes and we frequently do! This fallibility is an important concept for the critical realist (Collier, 1994). In fact critical realism proposes that it is because we do make mistakes that we know that reality exists outside of our concepts of it (Danermark et al., 1997). It is this explicit fallibility that gives critical realism the advantage in how it supports the development of knowledge. It allows us to be bold in our wondering but cautious in our theorising.

We know as realists that all we do must be fallible, and so we must ensure that what we do is also corrigible. We know that we are going to make mistakes, we know we are going to be wrong, so we must help people as much as we can to see how we make mistakes and to see how we have been wrong. Hence the need to theorize explicitly, to model the processes or situations we are seeking to understand, to observe systematically. (Archer et al., 1999, p16). 
When we get things right we will not necessarily know that we have and some knowledge is more fallible than other knowledge. In the natural world where closed systems can be investigated we are more likely to be right. However even here there is the possibility that new knowledge will be discovered that will prove us wrong. For the critical realist this fallibility does not imply uselessness. Each attempt at getting closer to the world we experience helps the world to grow and to improve. This approach to fallibility is, I think, what marks critical realism as the 'third way' of research, crossing the positivist/ relativist divide, and encouraging us to challenge knowledge in order to develop as a species.

\section{Risk: Focusing the research}

In her discussion of the various approaches to risk, Lupton (1999) proposes that there are three epistemological positions from which risk might be investigated. There were the two contrasting positions that I have discussed above, the positivist and the relativist approaches. She calls them 'realist' and 'strong constructivist' approaches. She describes a position between these two that she rather negatively words as the 'weak constructivist' position, which is very similar to Bhaskar's critical realist approach. This perspective views risk as "... an objective hazard, threat to danger that is inevitably mediated through social and cultural processes and can never be known in isolation from these processes" (Lupton, 1999, p35).

Lupton identifies that both the 'risk society' and the cultural perspectives of Beck and Douglas are theoretical approaches associated with this position. She states that the key questions related to 'risk society' ask about the relationship of risk with the structure and processes of late modernity and how it might be understood in different socio cultural contexts. Given my position in this study, that risk in the world of the midwife is real and that how she deals with risk is strongly effected by the world in which she works, this study has significant epistemological and structural congruence.

Beck himself discusses the issue of his epistemological position. He has been accused of being a realist but has preferred to develop a position that is neither realist nor constructivist. Neither, he thinks is sufficient on its own. He says

I consider realism and constructivism to be neither an either-or option nor

a mere matter of belief. We should not have to swear allegiance to any 
particular view or theoretical perspective. The decision whether to take a realist or a constructivist approach is for me a rather pragmatic one, a matter of choosing the appropriate means for a desired goal (Beck, 1999, p134).

Later he goes on to state;

I am both a realist and a constructivist, using realism and constructivism as far as those meta-narratives are useful for the purpose of understanding the complex and ambivalent nature of risk in the world risk society we live in (Beck, 2000, p212).

He calls for pragmatic research not limited by any particular meta-narrative and a need to 'break out of the iron cage of conventional and orthodox social science and politics' using both imagination and discipline (Beck, 2000). This is why being a midwife is such a useful position to be in. I do not come from a clearly identifiable academic knowledge base. The notion of knowledge synthesis is more natural as we do it in practice all time. Beck states that risk breaks down the barriers between objectivity and subjectivity; reality becomes virtual. Risk also breaks down the relationship between past, present and future. The past loses its power to determine the present as we become focused on colonizing the future. He also sees that realism and constructivism are both useful approaches.

I feel strongly that we have to be imaginative yet disciplined if we are to break out of the iron cage of conventional and orthodox social science and politics. We need a new sociological imagination which is sensitive to the concrete paradoxes and challenges of reflexive modernity and which at the same time is thoughtful and strong enough to open up the walls of abstraction in which the academic routines are captured (Beck, 1999, p134).

Critical realism therefore provides a philosophical and epistemological springboard from which to leap into this research. What I attempt to do is to structure a research project that does not oversimplify the complex and multi faceted problem of risk in midwifery. For me, critical realism is an exciting approach. It has challenged me intellectually and has helped me to settle into my project with some confidence. I felt I did not need to be over-anxious about getting to the absolute truth of the matter, of being completely right. It allowed me to place my own perspective within the 
research and see to see the research more as an offering in the continued growth of knowledge, an offering open to challenge and reinterpretation.

\section{Where to go? Critical Realism and the research design}

The ontological basis of this research therefore is informed by the philosophical approach of critical realism. This research acknowledges that the world of the midwife is complex and multi-layered. Any attempt to describe or interpret it must reflect this. In my own terms and from my own experience in the 'real' world, I as a midwife deal constantly with multiple paradigms, worldviews, or truths. To choose to acknowledge this and to do so within a research project would, I consider, match the dilemmas of practice. I was determined that my research would reflect the real world whatever that might be and that it would provide, not a simple description but a contribution to the understanding of it, that it would uncover generative powers. The motivation for this thesis is in fact, exactly this dilemma of the interplay between many worldviews with which the midwife must deal. These worldviews are exemplified in how she deals with risk in general and the referral for obstetric consultation in particular. Baskar's work spoke to me immediately as having some potential for assisting me in this process.

In order to establish how the principles of critical realism are relevant to the research question I will now describe this research project with reference to its philosophical and practical applications. Archer et al. state:

For realist philosophy to really have methodological consequences, the link between the posited abstract ontology and the shape and texture of the research findings must be systematic and explicit. There must be a consistency between these two ends of the research process (Archer et al., 1999, p15).

Risk is a concept without concrete form and so is heavily theory-laden. This is evident in many of the strata that Bhaskar talks about. Different people understand it in different ways; psychology, anthropology, sociology and epidemiology all have their own interpretations of it, their own theories about it. Yet none may be 
considered truer than any other. They are incomplete and fallible yet are very helpful in leading us to a more complete understanding of the world. Theory, Archer says

... contributes both to clarifying the 'what' questions and to some initial strategies for trying to answer the 'why' questions, not in any final sense but in helping to build up an exploratory knowledge that purports to actually deal with the real (Archer et al., 1999, p12).

Theory has been an important part of this research, because of the very nature of risk; it is very theory laden. I have chosen to ground this research in socio-cultural theory, particularly that of Beck and his conceptual approach of ' risk society' and Douglas's ideas of blame and values.

Critical realism espouses three underlying principles in the design of research:

\section{All research should have generalising claims}

The usefulness of the data within research is that it ought to help us understand society. In order to do this there needs to some form of generalisation (Porter, 2002). Critical realism proposes two types of generalisability. In the first type, generalisation is an extrapolation from empirical data where events are assumed to be valid for a larger population. The event or phenomenon is seen as a generally occurring phenomenon or event and is established inductively (Danermark et al., 1997). This level of analysis relates to Bhaskar's empirical domain of reality and excludes the deep domain of what he calls 'the real'. The second type of generalisablitiy relates to this deep domain where knowledge of the constituent powers is generalised using what he calls 'abductive and retroductive' inference (Danermark et al., 1997).

\section{Inference is essential.}

Critical realism proposes four modes of inference: abduction, retroduction, as well as induction and deduction. Abduction is the process of interpreting and recontextualising phenomena within a new conceptual framework or set of ideas and its central concern is to attribute meaning. Abduction requires creativity and imagination on the part of the researcher. Retroduction proposes what the basic conditions are or must be, in order for the phenomenon to exist the way it does. It provides knowledge of the structures that cannot be directly observed within the domain of the empirical. The researcher must be able to abstract. There are no 
fixed criteria for assessing the validity of either abduction or retroduction. Within a research project it is sometimes difficult to separate them as they are often undertaken concurrently (Danermark et al., 1997).

\section{The aim of social science is to explain.}

This implies the need for both description and some understanding of the underlying generative mechanisms that might make things 'as they are'. This too requires the use of abduction and retroduction. Causality is complex, multilayered and emergent and cannot be investigated at an individual level. The varied stratum of the world are hierarchically organised and each has its own powers and mechanisms that build on the mechanisms of the underlying strata creating new structures. These complex interactions cannot be investigated or explained in a reductionist way. In the social world therefore there can be no predictability. The task of the social scientist is to explore their interaction. Explanation must attend both to structure and to the underlying mechanisms and any explanation that deals with either on its own is likely to be inadequate. Causation therefore will be multiple and complex due to the open and stratified nature of the world (Archer et al., 1999).

This research aims to explain how midwives make sense of risk. In order to do this some explanation of the underlying 'generative mechanisms' needed to be uncovered. Risk is operationalised as the referral for obstetric consultation, the place where risk and midwives' management of and attitudes towards it were most likely to be expressed. This was necessary as dealing with a highly abstract concept such as risk without a tangible context was unlikely to provide data of any depth. Complex social processes need to be simplified in order to be studied. (Danermark et al., 1997). How risk is expressed in midwifery practice is an excellent example of a complex reality. Archer et al state

The conceptualization of the relationship between abstract theory and the level of the concrete is also valuable. It is imperative, rather than tending to forget the real world and produce theorization after theorization in the abstract, to continually revise one's theory in relation to the analysis of the concrete and redefine the concrete in terms of the theoretical advances that have been made (Archer et al., 1999, p12). 
This has also been discussed by Alaszewski and Alaszewski (1998), who commented that general questioning about risk as an abstract concept led to responses that were bland and uninformative. Critical realism supports a position in which these concrete experiences can then be built back up into theory. It provides a philosophical place to move beyond the examination of specific social instances, in order to examine the general structural context of those instances (Porter, 2002). It facilitates the movement between the concrete world and abstract theory. In the case of this research I have identified risk as the concept I wish to study, 'risk society' as its theoretical umbrella and midwives referral for obstetric consultation as the operationalised or 'concrete' form.

The referral for obstetric consultation also reflects the dialectical nature of the position taken. This place is an expression of where risk is most dynamically expressed. It is where risk meets medicine and the institution, and where it challenges what the true purpose of midwifery is. This is where the tension is most explicit.

\section{Intensive and extensive research methodologies}

There is no one particular research methodology associated with critical realism. Instead critical realism establishes guidelines for the use of existing methods. It advocates that to get as close as possible to the nature of reality multiple lenses should be used, as there can never be only one view. This also means that a question is in a sense never truly answered and that the more research from as many perspectives as possible is desirable. There may always be more to know and you might have got it wrong.

Questions of method then are essentially practical, pragmatic questions (Sayer, 1992). What is most important to consider is that method, object and purpose should be considered together

If we assume - as critical realism does - that social science studies are conducted in open systems, that reality consists of different strata with emergent powers, that it has ontological depth, and that facts are theoryladen, then these are some factors that affect the choice of design and method (Danermark et al., 1997, p150). 
Given these assumptions, neither an objectivist nor a subjectivist study will suffice. Within traditional social science the two main methodological approaches are quantitative and qualitative. Historically the quantitative approach has reflected the objectivist view and the qualitative, the subjectivist view. Danermark, Ekstrom et al (1997) propose that this terminology reflects a dichotomous, either/or approach, an approach that has caused much debate in the research community. In order to provide an alternative, one which fits more aptly to the critical realist approach they use the concepts of extensive and intensive research methods and see research as an ongoing interaction between them. This terminology then reflects a more inclusive and relational approach to their use. Archer, Sharp et al (1999) also propose that the realist researcher must be a 'methodological pluralist'. This mixed method approach then, enables triangulation as the different perspectives are integrated. "This is the combination of the results of two or more rigorous studies [are] conducted to provide a more comprehensive picture of the results than either study could do alone" (Morse, 2003, p190).

The two methodological lenses can both support and challenge each other. They can give strength and depth to the findings and the analysis adding a third dimension not available if each is undertaken alone. Table 3.1 summarises the main characteristics of the two methodological approaches. 
Table 3.1 Characteristics of intensive and extensive methodological approaches

\begin{tabular}{lll}
\hline & Intensive & Extensive \\
\hline Research question & How does a process work & What are the regularities, \\
in a particular case or common patterns, & distinguishing features of a \\
& small number of cases/ & dhat produces a certain population? \\
& change? What did the How widely are certain \\
& agents actually do? & characteristics or processes \\
distributed or represented?
\end{tabular}

Source: Table 6 in (Danermark et al., 1997, p165)

\section{Choosing the methods}

This piece of research contains two methods, one an extensive and the other an intensive procedure. The extensive part of the study consists of a national survey of midwives' referral for obstetric consultation practices. This was to 'uncover the regularities, the common patterns and distinguishing features' of midwifery practice and attitudes associated with referral. In particular I wanted to discover how often midwives make referrals, to whom and for what. I also wanted to know whether 
midwives with different characteristics varied in the way they referred and to ascertain some of their attitudes towards referral. In practice, the decision on which extensive research method to use is usually reasonably self evident and straightforward. My decision to use survey in this research reflects this. The decision about which intensive method to use is more problematic as there are many methods possible within the intensive approach.

I decided to use focus groups in order to obtain the intensive data. A focus group can be defined as

...a carefully planned discussion designed to obtain perceptions on a defined area of interest in a permissive, non-threatening environment. It is conducted with approximately seven to ten people by a skilled interviewer. The discussion is relaxed, comfortable and often enjoyable for participants as they share their ideas and perceptions. Group members influence each other by responding to ideas and comments in the discussion (Krueger, 1994, p18).

My decision to use focus group discussions was made for a number of reasons, some of which related to my own place in the research and others to what I saw were some of the benefits that focus groups can offer.

Focus groups seemed such a natural research process for midwifery; women with women talking, sharing, crying or laughing, usually accompanied by good coffee and something to eat. Wilkinson (1999) recommends focus groups for research because they look at interaction within the social context; they are contextual. This is certainly true for midwives. They are used to getting together to reflect on their practice. It is a normal part of many midwives' lives. Wilkinson also says that focus groups have the potential to be non-hierarchical; that the group itself can have considerable impact on how the research progresses; the group takes on a life of its own and the facilitator can be much less obvious in the process. They also have value as consciousness raising and collective sense-making, as meanings are shared and negotiated. The participants can reflect on their own experience and attempt to make sense of them in the light of others'. This does not mean that consensus must be reached. This not need be the aim. 
Given all these potential benefits I was surprised to discover that it is a little used method in feminist research (Wilkinson, 1999). I also chose focus groups as opposed to individual interviews so that midwives might benefit by participating in the research. I thought that the group process would enable the sharing of perspectives and might provide information that I would otherwise not have considered. "The fact that group participants provide an audience for each other encourages a greater variety of communication that is often evident within more traditional methods of data collection" (Kitzinger, 1994, p108).

I planned to leave plenty of room for interaction, to let the participants hear the voices of others. I also planned that the descriptive data from the survey would be presented towards the end of the discussion as one way of providing feedback to the participants. I also planned to ask the midwives whether there were any questions that they would like me to investigate from the data; I wanted them to have some input into the analysis. For this reason the focus groups were conducted after the survey and after the descriptive data from the survey had been partly analysed. Having the focus groups situated after the survey meant that I could use the focus groups to help in the interpretation of the survey (Stewart \& Shamdasani, 1990).

My own experience was also an important factor in choosing this method. As a midwife teaching at post-graduate level I have considerable experience in facilitating group discussion and have great faith in the ability of the process both to draw out valuable perspectives and to enable the group members to gain insight both into their own and others' perspectives, to learn and to grow. Personally, I felt the need to stay connected with the practice world and the practitioners, the need to stay 'real' and I thought that this would be a place where I might be able to do a bit of sharing too. There was the possibility of my participating in the experience.

Focus groups were also chosen as opposed to individual interviews as the research question aims not to explore individual's perspectives but to understand how midwifery as a profession deals with risk in practice. This was also reflected in the theoretical approaches I chose to present; I did not present psychological theory but socio-cultural approaches which seek to understand risk in terms of group meaning. It seemed appropriate therefore to seek group meaning within a group context. 
Waterton and Wynne(1993), in their study of community attitudes to risk, also decided to use focus group research in addition to survey. They commented that current socio-cultural definitions of risk cannot be divorced from the institutional context, the historical context, current contextual issues, self-esteem and identity, and a sense of agency or power, and that focus groups were a particularly apt tool in which to capture contextual issues of importance. They commented, "Focus groups can offer us a telling snapshot of attitudes-in-the-making, of the way in which people manoeuvre and negotiate around the difficult issue of living with and talking about risks" (Waterton \& Wynne, 1993, p142).

The data gathered from focus groups is of a different nature from that of individual interviews. Both focus groups and individual interviews have the potential to obtain rich data in the participant's own words enabling the researcher to explore deep levels of meaning (Stewart \& Shamdasani, 1990). Individual interviews facilitate this depth at an individual level. The researcher might ask 'What do you think that is about?' Within a focus group it is an understanding of group norms and group process that are more central. Also this gives “...intersubjective testing to eliminate the idiosyncratic, personal components of the insights - to find some but not absolute consensus amongst a group of practitioners" (Reason \& Goodwin, 1999, p293).

The researcher might ask 'What do the rest of you think about that?' Focus groups are not just a lot of interviews at the same time. They are distinguished from individual interview by the explicit use of the group interaction and the group norms as research data (Kitzinger, 1994). This is important to consider when analysing the data.

In this research the extensive data are analysed and interpreted statistically and the intensive data thematically. Abduction and retroduction were then undertaken incorporating both sets of data, using creativity, imagination and reflection, to produce an explanatory model of midwives' relationship with risk. Detailed design and process of the extensive and intensive procedures of the research are included in chapters 4 and 7 respectively. 


\section{Putting the two together}

There are many different ways of undertaking and presenting mixed method research. Morse (2003) presents eight different types of mixed method design, some of which are determined by a deductive approach and others by an inductive approach. This piece of research seeks to discover how midwives make sense of risk in practice and has taken both a deductive approach (survey) and an inductive approach (focus groups). Morse suggests that one or other of the methods will be dominant within the research; that research cannot be informed equally by inductive and deductive studies. Although I would agree with her in one sense, in the sense that the overall aim of this research is inductive, I consider that within this research project, the more deductive survey is not less dominant than the more inductive focus group discussions. They each provide a different lens through which to view the issue. It is in the implications or utility of this research where dominance may be more of an issue, in particular for the reader or user of this research. What I have become aware of in undertaking this research is that description itself, in this case the act of describing what midwives do, is a very powerful tool in the act of uncovering underlying mechanisms. Perhaps the issue is in the eye of the beholder and is primarily one of value over fact. For example I expect that the results of the survey may be dominant in some contexts and that the thematic analysis may be of more interest in others.

There are however issues of dominance within each part of the study. In asking the question about how midwives deal with risk, I have proposed that both what they do and how they think must be examined. 'What they do' is dominant in the survey and 'what they think about it' is dominant in the focus group discussions. However both procedures deal with both questions. The challenge then is how these different approaches might 'talk to each other' and in how they might be interpreted and presented in a way which is coherent and theoretically sound. Within this project I present the two methods in the same order in which I undertook them. However rather than providing a separate interpretation for each component and then merging them, I have built the interpretation as the project proceeds.

The piece of extensive research, the national survey was undertaken first. Chapter 5 presents the complete results of the national survey. In Chapter 6 the deductive 
implications of the survey findings are discussed, but without an examination of the in depth implications of the midwives attitudes. These are included alongside the interpretation of the focus group data in Chapter 8 , the rationale for this being that discovering the midwives attitudes was the dominant component of the focus groups. It made pragmatic sense to include them here. Chapter 7 describes the design and process of the focus group discussion. Chapter 8 provides the first level identification of the inductive, coded themes. Chapter 9 contains the model development. This is where the final phase of the analysis, the abduction and retroduction are presented. In presentation terms what has eventuated is that the quantitative data is examined on its own in more detail than is the qualitative data. However the analysis of the qualitative data is dominant in the final, aggregated analysis. In this way both data sets are thoroughly examined.

\section{Ethics}

There are particular ethical implications for this project. Social science research deals not with physical intervention as such, but never-the-less does intervene to varied degrees in peoples lives. All facts and all action are inherently value-laden and have the potential to impact positively or negatively. This applies to research no less than it does in the everyday world

\footnotetext{
Social science investigates an inherently value-charged world of social phenomena, positions, roles, identities and relations, whose meaning and significance always to some degree involve evaluations of good and evil, right and wrong, and so on. When social science brings into question and analyses everyday knowledge it therefore risks not only getting into conflict with alternative experiences and concepts, but also with deeply felt values and ethical guidelines (Danermark et al., 1997, p25).
}

The ethical implications of this research relate primarily to the protection of the participants and to the integrity of the research process. The detailed ethical issues related to the extensive and intensive procedures are provided in Chapters 4 and 7 , where the design and process of each of these aspects of the study are presented in detail. As this was a national study, ethical approval was required and received from 
the Victoria University of Wellington Human Ethics Committee and each of the 23 Regional Health Ethics Committees in New Zealand (Appendix A).

\section{Summary}

Midwives place themselves with women during the journey of birth. A central part of their place is also with risk. This research seeks to discover how they work with women and with risk within current techno rational and socio cultural constructs of risk. Risk for midwives is both real and constructed. Critical realism provides a philosophical approach that can support both the research question and midwifery knowledge. It proposes that reality does exist and that it is multi-layered and complex. Knowledge of this reality at the deep causal level is possible, fallible but necessary if we are to improve the human condition. Many lenses or multiple approaches to investigation are recommended. Risk, in this research is operationalised as the referral for obstetric consultation and is examined both extensively and intensively. A national survey of midwives' referral practices and attitudes was conducted, followed by focus groups with midwives. The next chapter begins to describe the research process by introducing the first part of the research, the national survey. 


\section{Chapter 4: The Survey - Design and Process}

\section{Introduction}

This chapter presents the design of a national survey of New Zealand LMC midwives, conducted in 2001. The survey examined practice which occurred during the latter part of 2000. The aims and objectives of the survey are presented, followed by the detail of the design. This detail includes the selection of the survey population and the representativeness of the respondents, the design and pre-testing of the questionnaire, and the processes of data analysis. Issues of reliability and validity are also addressed.

\section{Why a survey?}

A survey was conducted as the research question sought to explain how midwives make sense of risk as expressed in their referral practice and process. It was therefore important to discover what their referral practices were and a survey was the logical way to obtain this information. It also provided an opportunity to discover some of their attitudes in a quantitative form, which would prove useful alongside the qualitative data.

\section{Aims and objectives of the survey}

This research proposes that the process of referral for obstetric consultation is a practice where risk may be operationalised. The referral practices of New Zealand midwives are unknown so the main aim of the survey is to describe them. As the survey seeks to do this within the theoretical context of a 'risk' environment there was opportunity within the survey to discover midwives' values, beliefs and attitudes particularly relevant to risk and its management.

The objectives of the survey of New Zealand LMC midwives were:

- To describe the rate and reasons for obstetric consultation

- To describe the patterns of transfer of clinical responsibility to the secondary care maternity service 
- To describe the extent to which midwives continue to provide care for women who have had the clinical responsibility for their care transferred

- To determine the demographic characteristics, practice patterns and attitudes of the midwives which might impact on their referral practices

- To describe midwives' attitudes to referral including the referral guidelines, the interface between primary and secondary maternity care, and risk management

- To determine the extent to which attitudes to risk and its management are related to the environment in which midwives work

\section{Planning for the survey}

It was clear from the outset that the amount of information required was both extensive and complex. In keeping with the philosophical approach of critical realism and the theoretical approach of 'risk society', it was important to consider that these referral processes occur within an open and dynamic system and within a particular cultural context. It was important that both the design and analysis of the survey would lead to cautious interpretation that would not be oversimplified or reductionist. The strength of this survey is in its power to describe.

\section{The study population}

The population identified for this study consisted of midwives who were Lead Maternity Caregivers (LMC). LMC midwives are responsible for the assessment of the woman's needs, the planning of care and for ensuring the provision of maternity services including the referral for obstetric consultation (Ministry of Health, 1996). They, or, if they are employed the organisation they are employed by, hold and control the primary maternity budget. Accessing a database which identified midwives by LMC status proved to be problematic. Databases in New Zealand at the time classified midwives as self-employed, independent or as case-loading. These midwives need not necessarily have been LMC midwives as they may have been working in a shared care arrangement with doctors. In shared care it is usually the doctor who is the LMC. The databases that recorded self-employed, or independent midwives did not identify LMC midwives employed in hospitals. Any database used 
would therefore have under-reported employed LMC midwives and over-reported independent midwives not providing a LMC service.

It was estimated by the Health Funding Authority that the practising LMC midwifery population at the time of the study was approximately 900 (Health Funding Authority, 2000). It was therefore feasible to conduct a total population survey of LMC midwives. The difficulty was in accessing the contact details for this population.

There were three possible databases to access: the Nursing Council of New Zealand, Health Benefits Ltd and the New Zealand College of Midwives (NZCOM). The Nursing Council, the registering body for nurses and midwives in New Zealand at that time, had the most complete database, although the Council's information analyst related that she thought their database might not be accurate for that year. This database identified midwives by caseload rather than by LMC status and also had a category for midwives working in a hospital, some of whom may also have been providing an LMC service. The Nursing Council does not release the database but are prepared, at cost, to post out questionnaires. There is no ability to identify nonresponders, so a second post-out would need to have included all midwives and would have been expensive. Midwives on this database are able to veto access for research purposes.

The second possible source was Health Benefits Ltd., the funding body for those midwives proving care under the Section 51 contract. In 1999, 929 midwives made at least one claim for funding through this organisation (Health Funding Authority, 2000). This database does not include employed midwives or those who are employed by a hospital or health service funded by contracts other than Section 51. At the time of the survey there were 20 organisations holding non-Section 51 contracts (Health Funding Authority, 2000). This database was not accessible for the distribution of research material.

The next possible source of midwives was the New Zealand College of Midwives (NZCOM). Only midwives who belong to the College are included in this database. However all midwives not employed by hospitals gain their indemnity insurance via NZCOM so there is a high rate of membership. It was thought that this may be the most complete and current database. The College were also prepared, after gaining 
approval from their National Committee, to provide the names and addresses of their case-loading midwives. Their database did not distinguish between case loading and LMC midwives. Midwives who belonged to the College were also able to veto their details being given out. There were 474 midwives who were case loading and who had not vetoed their details being released and this is the database that was selected as the basis for this study. Attempts were made to increase the sample. Personal contact was made with every hospital offering a secondary maternity service requesting the names of all LMC midwives employed by their service including those employed in the primary birthing units.

In order to further extend the database every regional telephone directory, and all New Zealand midwifery websites, were accessed. Contact details of midwives less likely to be NZCOM members were obtained through regional networks. These names were crosschecked with the NZCOM database to avoid duplication. The total number of midwives identified was ultimately 649 (Table 4.1). This will have included some midwives who, although they worked in a caseload model, would not have been LMCs. Thus they were not eligible for the study. It was important to be able to identify this in the survey.

Table 4.1 Sources of LMC midwives for survey database

\begin{tabular}{ll}
\hline NZCOM database & 474 \\
Contacting all secondary/tertiary hospitals & 97 \\
New Zealand telephone books & 32 \\
Websites & 33 \\
Regional Local networks & 13 \\
\hline Total & $\mathbf{6 4 9}$ \\
\hline
\end{tabular}

Although a total population survey was planned, the database was incomplete. As there were an unknown number of midwives providing an LMC service at the time, the degree to which this database is incomplete is unknown. Issues of representativeness of the responding population are important to consider and are examined later in this chapter.

Although not specifically addressed in this survey, it was apparent, when sample gathering, that numerous hospitals were providing team midwifery rather than LMC care. The Maternity Services reference document also noted this as a problem and recommended “....assistance to those hospitals that are non compliant with the Section 
51 Maternity Notice to develop a workout plan to achieve compliance" (Health Funding Authority, 2000, p8). The extent to which this is still occurring needs to be assessed.

\section{Questionnaire design}

There were three sections of inquiry in the questionnaire (Appendix B). The first consisted of questions of attribute: the demographic and work practice patterns of the midwives. These were to be used in establishing the representativeness of the respondent population and in associational statistical testing. The second section contained questions of behaviour and related to the midwives' referral for obstetric consultation practices. Midwives were asked for information on each woman they had referred in a four-month period. The final section consisted of beliefs and attitudes related to the management of the referral processes and the environment in which the midwives worked. It was clear that the questionnaire would be large so it was important to select questions economically.

Some questions from previous research by Guilliland (1998) were utilised in order to build on existing knowledge (de Vaus, 1995). Guilliland's (1998) study is the only other published research to describe the practice patterns of New Zealand case loading midwives and presents data sourced in 1995. Her study was in 2 parts. The first requested information about the midwives and had an $82.6 \%$ response rate. The second part requested practice information and had a response rate of $38 \%$. Neither non-response rate bias nor representativeness of the respondents was addressed in her research, and standard deviations of means were not supplied. Because of the year of this study, and the low response rate of the second part, it was of less use in determining the representativeness of the sample of referred women in this study, but in conjunction with the other data sets does assist in creating an overall picture of midwifery in New Zealand.

The content and structure of the questionnaire was also informed by my own experience as an LMC midwife and as a practice educator and reviewer. 


\section{Demographic and work practice patterns of the midwives}

This section aimed to provide data about the midwives that would both assist in establishing representativeness of the sample and provide the detail of those variables which might impact on referral practices.

The variables chosen related to the characteristics of the midwifery population. Some of the questions were taken from the Guilliland's (1998) study. These related to country of initial registration, types of registration, present practice (with LMC added) and types of midwifery services offered. In order to identify the geographic location of the sample, the questionnaire asked the midwives to identify the secondary maternity facility to which they most commonly referred and the distance from this secondary care facility to their home. Finally the midwives' caseloads and numbers of referrals over a four-month period were requested.

\section{Information about the referral processes and outcomes}

In this section the midwives were asked to provide detail of the women that they had referred for an obstetric consultation over a 4-month period. Given the NZCOM's guidelines of 4-6 births per month (New Zealand College of Midwives Inc, 1993) and the rate of my own and my colleagues' experience of referral, this four month period would on average require the midwives to provide the data on five or six women. Guilliland's (1998) survey found the antenatal consultation rate was 30\% and the labour and birth consultation rate was $28 \%$. This data also indicated that, given an average caseload, midwives would need to fill in data from between four and seven women.

The questionnaire requested demographic detail of the referred women. There are regional differences related to accessing secondary maternity services. Some women are referred to private obstetricians, some to obstetricians working in the public hospitals and others to an obstetric team. If an antenatal referral was made, the midwives were asked whether or not they accompanied the woman to the initial antenatal consultation.

The questionnaire asked the midwives to provide the reasons for the referrals and provided coding on the questionnaire form. These codes were drawn from the 
Guidelines for Referral (Health Funding Authority Maternity Project Team, 1997) and from the diagnoses for antenatal admission from the Report on Maternity 2000 (New Zealand Health Information Service, 2003). These reasons were also compared with international guidelines in countries where there is midwifery-led care.

It was expected that there would be a large number of referrals for 'other' due to the large number of less common complications associated with childbirth. The Report on Maternity, for example, reported that $34 \%$ of admissions for severe complicating diagnosis and $65 \%$ of the admissions with moderate or no complicating diagnoses were for 'other' (New Zealand Health Information Service, 2003). Other studies which reported reasons for referral or transfer of care also reported a large number of reasons as 'other' (MacVicar et al., 1993; Wallace, Mcintosh, Brownlee, Laidlaw, \& Johnstone, 1995).

As the referral guidelines discuss transfer of clinical responsibility, this study sought to ascertain both the extent to which this was occurring and also the extent to which midwives continued care after clinical responsibility had been transferred. This section proved the most difficult as there are regional differences in the understanding of this practice. Lead Maternity Caregiver status was, in some regions, being linked to this transfer of responsibility. One reason for this is that the payment rules for secondary maternity services at the time allowed the hospital to claim the LMC fee where there was a transfer to secondary maternity services for an entire module (Health Funding Authority, 2000). Some hospitals were requiring a sign-over of LMC to the secondary care service, others not. Transfer of clinical responsibility was therefore differently interpreted and managed. Some secondary services supported the idea of shared clinical responsibility and others did not. A following question, about continued midwifery care once transfer of clinical responsibility had occurred, and whether payment was received for this care, aimed to provide some clarity about the extent of care which was either shared or 'taken over'. The questionnaire then asked for details of the birth outcome. This included whether the birth was normal, by ventouse/forceps, or by caesarean section, whether there was an epidural, and whether there was a perinatal death. 


\section{Midwives' opinions and attitudes}

The final section of the questionnaire sought some of the midwives' attitudes and opinions regarding the primary/secondary interface using a seven point Likert scale. This closed question approach was used to minimise the time taken to complete the questionnaire. Focus group discussions were planned to supplement this approach in order to provide more in-depth data (de Vaus, 1995). The Likert scale was seen as being able to get at specific aspects of the issues and to elicit the strength of opinion (de Vaus, 1995). Both the wording and positioning of the statements were carefully considered. Several complementary questions were developed in order to clarify aspects of the interface and to provide construct validity (Fowler, 1995). The scale was rated from strongly agree to strongly disagree and a central, neutral position was provided.

The choice of statements reflected the research questions. The central concern regarding the processes around the primary/secondary interface was developed, as were attitudes to risk and its management. There were three statements regarding the referral process and two of these related also to the referral guidelines. The first stated that the pattern of referral was appropriate to the needs of the women they cared for, the second that the guidelines required referral more than necessary and the third that they were a useful tool.

As the quality of the relationship between the primary and secondary maternity service relates both to the relationship with obstetricians and also with the service in general, two statements concerned this issue. Central to the research question was the role LMC midwives play in caring for 'at risk' women. Midwives were asked whether or not they agreed that this was appropriate practice. They were then asked to give an indication of how important risk management was to their practice. The final statements concerned the medico-legal environment. These two statements related to how the environment affected safety for women and the degree to which it restricted the midwives' practice.

\section{Pre-test}

There were many drafts of each section of the questionnaire and it was important to ensure that the questions were relevant at a national level. The questionnaire was 
discussed with both the NZCOM and with the Maternity Manager from the Ministry of Health. The questionnaire was pre-tested with six midwives. Although this number is not large this decision was made in order to minimise the loss of possible research participants in the total population surveyed. Representativeness was attempted by selecting midwives from different areas and practice patterns. The midwives worked in three different areas. Two of the areas had access to a tertiary maternity unit supportive of LMC midwives. The other area had a secondary maternity facility that was seen as less supportive. The midwives worked in different models of care. All provided an LMC midwifery service and two were employed. One midwife did some of her work in a shared care model and all had a variety of caseloads. They were asked to record how long it took to complete the survey, to write any comments they wanted on the questionnaire form and to highlight questions that were confusing.

Several of the midwives who commented, said that it was a stimulating process, which got them thinking about how and why they made referrals. Their comments and suggestions related to the contents of the covering information letter and the reasons for referral. It took them between 20 and 40 minutes to complete the task and all needed to access their clinical notes for client detail. They did not rely on memory. Following the pre-test there was some reworking of the information letter to improve the clarity of the description of the study and some minor adjustments to the 'reasons for referral' section of the questionnaire. The most significant change was made to the questionnaire section related to transfer of clinical responsibility, which had originally focused on change of LMC status. Following discussions with the NZCOM and with the Ministry of Health's Maternity Manager it was apparent that this would have been confusing in regions where transfer of LMC did not occur. The question was therefore focused on transfer of clinical responsibility.

\section{Distribution}

The first post-out occurred on 29 May 2001 and the second, on 17 August 2001. Included with the questionnaire was a covering letter, which explained the research and the questionnaire (Appendix C). There was a tear off section for the midwife to fill in which asked for her name and contact details and whether she wished to participate in a focus group should one be held in her area. This section, if completed, entered the midwife into the prize draw of two midwifery texts and enabled 
identification of who would require a reminder. The second post-out was sent to all midwives who had not returned the questionnaire or, if they had returned it, had not included their names.

A second insert, included in both post-outs, was of brightly coloured paper and encouraged the midwife to return the questionnaire even if it was not completed. Several options for non-completion were given, including 'ineligible', 'duplicate or 'didn't want to do it (Appendix C). A pre-paid, addressed envelope was included.

\section{Response}

In order to maximise the response several tactics were utilised.

- The questionnaire was as small as possible and was on a single piece of paper

- The questionnaire was pre tested for clarity and ease of completion

- A reminder was sent out. This included another questionnaire

- My 'insider knowledge' meant that I believed the issue would of interest to the midwives

- The timing of the survey was planned so that midwives had not recently been asked to participate in any other work related surveys

- The midwives were encouraged to return the questionnaire, even if it was not completed and were asked the reasons for non-completion

- A prize draw of two current midwifery texts was offered

- Anonymity was guaranteed

- A reply paid envelope was included

(Edwards et al., 2002; Glascoff, 2001)

When the questionnaires were returned, the tear off section with the midwives' details was immediately separated by a research assistant so as to protect anonymity. In total $433(66.7 \%)$ of the midwives responded to the survey and $311(47.9 \%)$ had completed the questionnaire (Table 4.2). Eighty-one of these respondents were not eligible as they were not providing an LMC service. The response rate was calculated by dividing the number of completed eligible responses by the number of known total eligible midwives and multiplying this by 100 (Fowler, 2002). The known ineligible midwives (81) were subtracted from the total sample (649). The final response rate for 
the survey was $56.47 \%$. This is a conservative estimate, as some of the nonresponders may also have been ineligible.

Table 4.2 Survey response

\begin{tabular}{|c|c|c|c|c|}
\hline & $1^{\text {st }}$ post-out & 2nd post-out & Total & Total $\%$ \\
\hline Sent out & 649 & $491 * * *$ & & \\
\hline Returned & 298 & 135 & 433 & $66.7 \% *$ \\
\hline Completed & 220 & 91 & 311 & $47.9 \% *$ \\
\hline \multicolumn{5}{|l|}{ Reasons for non-completion } \\
\hline Ineligible & 54 & 27 & 81 & $19.8 \% * *$ \\
\hline Didn't want to do it & 9 & 10 & 19 & $6.4 \% * *$ \\
\hline Duplicate/wrong address & 6 & 7 & 13 & $2.6 \% * *$ \\
\hline No reason given & 9 & 0 & 9 & $1.8 \% * *$ \\
\hline
\end{tabular}

\section{Responder representativeness}

Although there is no agreed-upon standard for an acceptable response rate (Fowler, 2002), within the medical literature published surveys of physicians have a mean response rate of only $54 \%$, and those of non-physicians have a mean response rate of 68\% (Asch, Jedrziewski, \& Christakis, 1997). Asch et al comment that researchers, editors and readers should devote more attention to assessments of bias, and less to individual response rates. This is in order to increase the external validity or generalisability of the results.

Given the response rate of this study, it is important to address issues of non-response bias and of representativeness. Non-response bias can occur when it is assumed that non-responders have the same characteristics as those that do respond. Two parameters that might be considered as having an effect are basic population characteristics and the distribution of the population (Fogliani, 1999). Within the design of this research it is not possible to assess the population characteristics of the non-responders but it is possible to assess their distribution (Figure 4.1).

Representativeness in this survey can be determined by comparing the characteristics of the respondents and their clients with the characteristics of the general population of case-loading midwives and childbearing women. To do this the data from the New Zealand Health Information Service (New Zealand Health Information Service, 2004) 
and from the Guillland (1998) study were utilised. Characteristics of the midwives that could be compared included age, years of experience, country of registration and proportion of direct entry midwives (Table 4.3).

In the absence of accurate national data on the location of LMC midwives the regional spread of the respondents is compared with the numbers of births in each region (Figure 4.1).

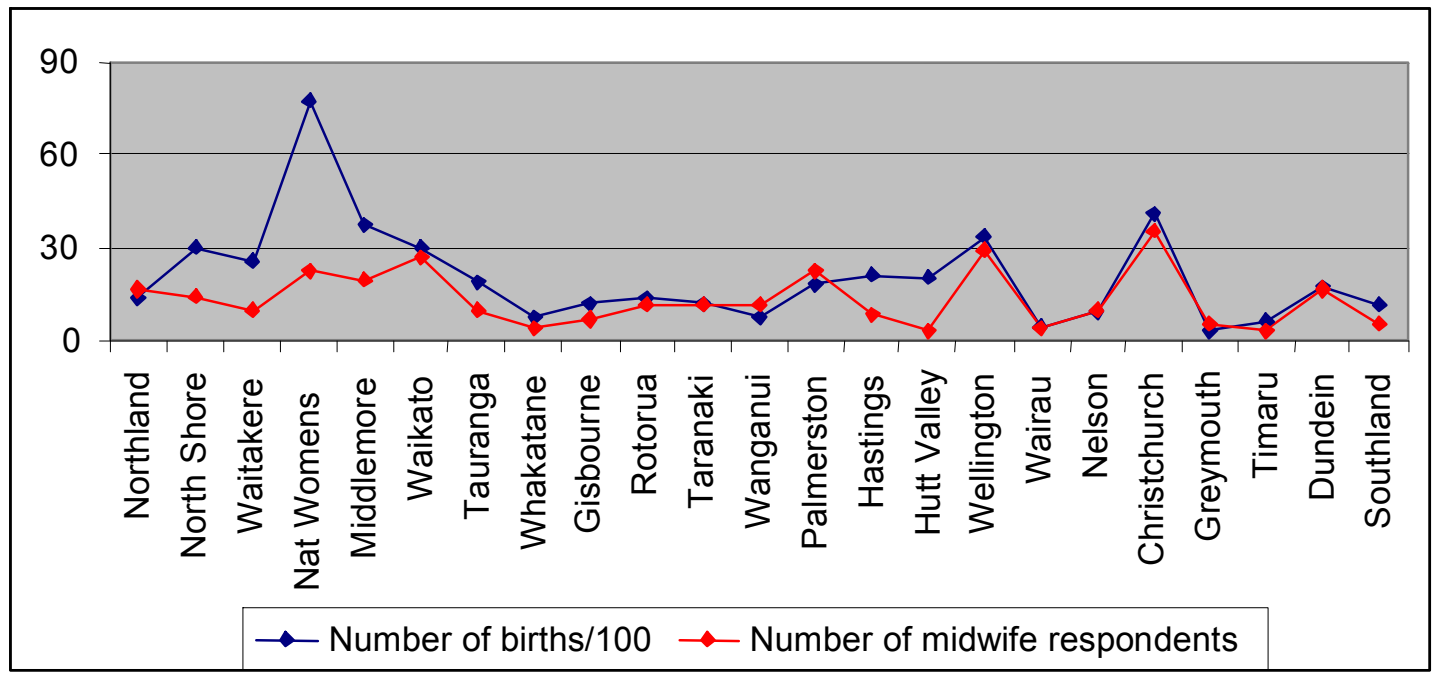

Figure 4.1 Facility location of midwife respondents compared with the national birth rate by location

Table 4.3 Comparison of midwifery demographic data with national data

\begin{tabular}{cccc}
\hline & Study data & $\begin{array}{c}\text { NZHIS } \\
\text { 2000 data }\end{array}$ & $\begin{array}{c}\text { Guilliland } \\
\text { 1995 data }\end{array}$ \\
\hline Midwives Mean Age & $43.7 \mathrm{yrs}$ & $45-49 *$ & $43 \mathrm{yrs}$ \\
Years of practice & $13.1 \mathrm{yrs}$ & $* *$ & $10 \mathrm{yrs}$ \\
Country of registration - NZ & $61.9 \%$ & $72 \%$ & $63 \%$ \\
UK & $24.8 \%$ & $15 \%$ & $24 \%$ \\
Aust & $8.7 \%$ & $3 \%$ & $9 \%$ \\
Direct Entry Midwives & $18.0 \%$ & $7.3 \%$ & $* *$ \\
\hline
\end{tabular}

* Mean not available

**Information not available

The distribution of midwives closely matched the distribution of births by hospital. The exceptions were Auckland, (National Women's Hospital in particular), the Hutt Valley and Hastings. These inconsistencies may be accounted for in that National Women's hospital provides maternity care for the most complex and acutely ill 
pregnant women in New Zealand. It would be expected therefore, that there would be less LMC midwifery in this hospital as a proportion of the total number of births. The Maternity Report also indicates that the proportion of LMC midwifery is low in the other Auckland hospitals and in Hastings and the Hutt Valley (New Zealand Health Information Service, 2003). It would be expected therefore that the ratio of births to LMC midwives in these three areas and thus in my sample would be larger.

As with the midwifery population, the characteristics of the women in this study were compared with the characteristics of the total childbearing population for the year 2000 taken from the Maternity Report (New Zealand Health Information Service, 2003) and from the Guilliland (1998) study (Table 4.4).

The client data taken from the Maternity Report indicates that the characteristics of the referred women in this study closely resembles that of the general childbearing population. Age and parity are closely matched. There are smaller proportions of Maori, Pacific and Asian women. The Maternity Report states that LMC midwives are more likely to care for teenagers and Maori and less likely to be LMC for Pacific and Asian women. The discrepancy between the number of Maori women in the national database and the number in this study can be accounted for by the smaller proportion of Maori women who have interventions in childbirth and would therefore have required a referral (New Zealand Health Information Service, 2003).

There are several difficulties with the accuracy of the information from the 'Report on Maternity' figures. The most significant one related to this study is that in the year $2000,30 \%$ of New Zealand women received care from LMCs who were not covered by the Section 51 contract. They were part of group contracts separate from Section 51 and the data they provided was aggregated and was unable to be merged with national data. A significant part of the antenatal and some intrapartum information therefore is missing. It was not possible to statistically determine whether the differences between the study populations and the total populations were significant due to the large difference in population size. 
Table 4.4 Comparison of details of referred women from study with other national data

\begin{tabular}{|c|c|c|c|}
\hline & $\begin{array}{l}\text { Study } \\
\text { data }\end{array}$ & $\begin{array}{l}\text { Report on } \\
\text { Maternity } \\
2000\end{array}$ & $\begin{array}{l}\text { Guilliland } \\
1995 \text { data }\end{array}$ \\
\hline \multicolumn{4}{|l|}{ Client Data } \\
\hline Mean Age & $29 \mathrm{yrs}$ & $29.3 \mathrm{yrs}$ & 28 \\
\hline Teenage births & $7.1 \%$ & $6.9 \%$ & $*$ \\
\hline Maori & $16.7 \%$ & $19.9 \%$ & $17 \%$ \\
\hline Pacific Peoples & $5.0 \%$ & $10.1 \%$ & $4 \%$ \\
\hline Asian & $4.0 \%$ & $6.6 \%$ & $2 \%$ \\
\hline Parity -0 & $32 \%$ & $37.2 \%$ & $34 \%$ \\
\hline Parity -1 & $31 \%$ & $31.7 \%$ & $*$ \\
\hline Parity -2 & $18 \%$ & $16.6 \%$ & $*$ \\
\hline Referral for & & & \\
\hline \multicolumn{4}{|l|}{ Consultation } \\
\hline Antenatal referral & $23 \%$ & $*$ & $30 \%$ \\
\hline Intrapartum referral & $20 \%$ & $*$ & $28 \%$ \\
\hline Postnatal referral & $2 \%$ & $*$ & $14 \%$ \\
\hline \multicolumn{4}{|c|}{$\begin{array}{l}\text { Transfer of Clinical } \\
\text { responsibility }\end{array}$} \\
\hline Antenatal & $4.1 \%$ & $*$ & $6.3 \%$ \\
\hline Intrapartum & $9.5 \%$ & $*$ & $14.2 \%$ \\
\hline Postnatal & $1.0 \%$ & $*$ & $1.6 \%$ \\
\hline \multicolumn{4}{|c|}{$\begin{array}{l}\text { Birth Outcome - Where } \\
\text { original LMC is midwife }\end{array}$} \\
\hline Normal birth & $83.8 \%$ & $71.8 \% * *$ & $82 \%$ \\
\hline $\mathrm{C} / \mathrm{S}$ rate & $11.8 \%$ & $16.6 \% * *$ & $8.3 \%$ \\
\hline
\end{tabular}

* The data are either not available or not applicable from this source ** $30 \%$ of the data are missing

Even given the limitations of these data sets there is sufficient information here to indicate that the sample of referred women in this study is representative of the general population of LMC midwives and childbearing women in New Zealand at the time.

\section{Ethical considerations}

In order to undertake the survey, contact names and addresses of possible participants were needed. A full research proposal and a copy of the Regional Ethics Committee approval letter was submitted to the New Zealand College of Midwives with a request for access to the names and contact details of all case loading midwives on their database. This was approved and two sets of named and addressed labels were provided. Requests were made of each hospital for the names of midwives who 
provided a LMC service. Addresses were not required as their questionnaires were posted to them at the hospital. All but one gave me the names and the one that didn't, offered to distribute the questionnaires on my behalf. All other contact details were recovered from publicly available information. The final database will not be accessed by anyone else or used for any other purpose.

Full information on the study was included with the questionnaire (Appendix C). Participation was voluntary and participation implied consent. There was no identifiable information on the questionnaire. If the return slip with the midwife's contact details was included, it was immediately separated before the data were examined. The completed questionnaires were kept securely. The design of the study and the data analysis and interpretation have been supervised and will be examined. The results of the study will be presented as widely as possible throughout New Zealand. They have already been presented at 5 regional workshops and at 3 national forums. The primary data will be stored securely for 5 years.

\section{Data analysis}

The data were entered into two databases set up in SPSS. Separate databases were created for the midwives and for the referred women.

\section{Data entry and data cleaning}

The databases were checked and cleaned. The first 10 entries were checked before any further entry occurred. Once entry was complete all fields of every tenth questionnaire were checked. Every row of data was visually checked for input that was out of range. Frequency and descriptive analyses were undertaken on every field and were checked for incorrect or unusual entries. In total $17(0.18 \%)$ of the questionnaires were found to have had one or more incorrect entries. Fifty-five $(17.7 \%)$ of the questionnaires were checked completely.

\section{Descriptive analyses}

The survey generated both continuous and categorical data. Mean, median, range, standard deviation and $95 \%$ confidence intervals of continuous data were generated. The categorical data were described and percentages and 95\% confidence intervals 
generated ( $95 \%$ confidence interval $= \pm 2 \sqrt{ } \mathrm{pq} / \mathrm{n}$, where $\mathrm{p}$ is the percentage, $\mathrm{q}$ is $(100-p)$ and $n$ is the sample size).

Multiple response questions were collated and analysed in SPSS. In order to facilitate statistical testing new categorical variables were created by regrouping these multiple response questions. This was undertaken for place of registration (in New Zealand or elsewhere), type of registration (midwife only, or midwife with a nursing registration), and employment status (employed or self-employed). The distance from the midwife's home to the hospital was regrouped into urban and rural as this data, although continuous, had a large number of outliers. Midwives' ages, caseloads, referral rates and caesarean section rates were computed using SPSS. Midwives' years of experience and caseload were transformed into grouped categorical variables for some of the analyses.

There was a small amount of missing data throughout. This is presented with the results as each variable is described. The only sizable amount of missing data was from the 25 midwives who, although they completed the bulk of the questionnaire, did not provide the detail of the 66 clients they referred. Descriptive statistics and percentages of the referral information relates only to those 1,408 women whose information was provided.

\section{Factor analysis}

Principle component analysis of the attitudinal scores was undertaken as several questions were closely related and it was thought that this may clarify statistical analysis. Three factors were identified. However Cronbach's alpha identified only one of the factors as having reliability even after progressive removal of variables. The factor that is included in the analysis relates to the degree of support in the primary/secondary interface and merges the two items - 'In my region there is excellent collaboration between primary and secondary maternity services' and 'Obstetricians in my region in general support continuity of care' (Cronbach's alpha .71). All other attitudinal variables are used in analyses separately. 


\section{Relationships between the referral rate, the demographic characteristics, the practice patterns, and attitudes of the midwives}

In order to determine whether any demographic characteristics, patterns of practice or attitudes of the midwives impacted on the referral rate, and to ascertain the extent to which attitudes to risk were related to the environment in which midwives work, further statistical analyses were undertaken. These analyses explored the relationships between these characteristics and compared them with the various groupings of the midwives (Voelk1 \& Gerber, 1999).

Where the continuous data was normally distributed, parametric testing was used. This continuous data included the referral rate, the midwives' age and the attitudinal scales. Where continuous data was not normally distributed non-parametric statistical tests were used. The parametric test used was the Pearson product-moment correlation and the non-parametric test was Spearman's rank order correlation.

Independent sample t-tests and one-way between groups ANOVA was used where the dependent variable was continuous and normally distributed. The Mann-Whitney U and the Kruskal Wallis tests were used where the dependent variable was continuous but not normally distributed.

A linear regression was undertaken to ascertain the effects of associated demographic variables on the referral rate.

\section{Summary}

This study aims to provide a comprehensive description of New Zealand LMC midwives' referral for obstetric consultation practices and attitudes. This national, total population survey had an acceptable response rate and was representative of the total population. The following chapter provides the findings of the survey and the results of the statistical testing that endeavoured to explore relationships between the variables in the study. 


\section{Chapter 5: The Survey Results}

\section{Introduction}

This chapter presents the results of the national survey of LMC midwives and their referred clients. The results are presented in three sections. The first section presents the demographic and work pattern data of the midwives, followed by the demographic details of the women who had a referral for obstetric consultation. This is followed by the details of the referrals. The information relating to whom the referral was made and the reasons for the referrals are described. Details of the transfer of clinical responsibility and ongoing care by the midwives are then presented.

The second section consists of the responses of the midwives to a variety of statements reflecting attitudes towards their referral processes, to caring for 'at risk' women, and to risk management in general. The third section presents the results of the statistical investigations into what might impact on the referral rate and explores relationships between midwives' attitudes and demographic characteristics. Throughout this chapter missing data are indicated by stating the number of midwives (n) who responded to each question. Statistical significance is indicated both in the text and in the tables. 


\section{Section One: Description of the midwives, the referred women and the referral for obstetric consultation processes}

\section{The midwives}

There were 311 completed responses to this total population survey of 649 caseloading midwives. This is a response rate of $56.4 \%$. The data collected covers a 4month period from 1 September 2000 to 31 December 2000.

\section{Demographic detail of the midwives}

The mean age of the midwives was $43.7 \mathrm{yrs}$ (SD 8.4). The average length of time these midwives had been in active practice, either in full time or part time practice, was 13.1 years (SD 8.8). Although the majority (61.9\%) undertook their midwifery education in New Zealand, a quarter were first registered in the United Kingdom and 8.7\% were educated in Australia. The remaining midwives were educated in South Africa, Europe, Asia or the Pacific. Eighteen percent of the midwives did not have a nursing registration. They were 'direct entry' midwives. All but three of the direct entry midwives were educated in New Zealand. Three quarters of the sample described themselves as self-employed, and half of these received their funding via some type of health provider organisation which managed the claims on their behalf. Ninety percent of the employed midwives were employed by a hospital. Table 5.1 provides a summary of their demographic and work pattern characteristics. 
Table 5.1 Demographic and work pattern characteristics of midwives

\begin{tabular}{|c|c|c|c|c|c|c|}
\hline & $\mathrm{N}$ & $\%$ & Mean & SD & Range & $95 \% \mathrm{CI}$ \\
\hline Age & 309 & & 43.7 & 8.4 & $23-66$ & $42.7,44.6$ \\
\hline Yrs of practice & 299 & & 13.1 & 8.8 & $1-35$ & $12.2,14.0$ \\
\hline Country of Registration & 310 & & & & & \\
\hline New Zealand & 192 & 61.9 & & & & $56.4,67.4$ \\
\hline United Kingdom & 77 & 24.8 & & & & $19.9,29.7$ \\
\hline Australia & 27 & 8.7 & & & & \\
\hline South Africa & 3 & 1.0 & & & & \\
\hline Europe & 3 & 1.0 & & & & \\
\hline Asia & 3 & 1.0 & & & & \\
\hline Other & 5 & 1.6 & & & & \\
\hline Type of registration & 311 & & & & & \\
\hline Nurses and midwives & 255 & 82 & & & & \\
\hline Direct Entry midwives & 56 & 18 & & & & \\
\hline Employment & 308 & & & & & \\
\hline Self Employed & 236 & 76.6 & & & & \\
\hline Employed & 72 & 23.4 & & & & \\
\hline Location* & 311 & & & & & \\
\hline Urban & 232 & 74.6 & & & & \\
\hline Rural & 79 & 25.4 & & & & \\
\hline $\begin{array}{l}\text { Types of midwifery ca } \\
\text { provided** }\end{array}$ & & & & & & \\
\hline Total midwifery care & 289 & 94.4 & & & & \\
\hline $\begin{array}{l}\text { Shared care with } \\
\text { doctors }\end{array}$ & 152 & 58.5 & & & & \\
\hline $\begin{array}{l}\text { Shared care with } \\
\text { hospital midwives }\end{array}$ & 11 & 3.6 & & & & \\
\hline Part care & 179 & 11.8 & & & & \\
\hline Other & 10 & 3.3 & & & & \\
\hline Main type of care provided & 308 & & & & & \\
\hline Total midwifery care & 276 & 89.6 & & & & \\
\hline $\begin{array}{l}\text { Shared care with } \\
\text { doctors }\end{array}$ & 20 & 5.5 & & & & \\
\hline $\begin{array}{l}\text { Shared care with } \\
\text { hospital midwives }\end{array}$ & 3 & 1.0 & & & & \\
\hline Part care & 9 & 2.9 & & & & \\
\hline Other & 3 & 1.0 & & & & \\
\hline Total clients booked & 280 & & 15.2 & 9.6 & $0-39$ & $14.1,16.3$ \\
\hline Annual Caseload & 280 & & 45.6 & 28.8 & $0-117$ & $42.2,49$ \\
\hline Individual referral rate*** & 247 & & 35.2 & 17.4 & $0-100$ & $33.0,37.4$ \\
\hline Overall referral rate $* * *$ & 247 & & 34.7 & & & $28.7,40.7$ \\
\hline
\end{tabular}




\section{Work patterns of the midwives}

The midwives were asked about the variety of ways in which they provided midwifery care. The survey had been aimed at those midwives who, at least in part, provided a lead maternity caregiver (LMC) service. Some midwives also worked in a shared care model with other practitioners such as obstetricians and general practitioners, and in these situations it is usually the doctor who is the LMC. Some midwives may also be LMC for only part of the maternity care such as in the postpartum phase, so the variety of models of care can be complex. It became apparent from the survey that a large number of midwives $(62.1 \%)$ also provided care alongside other practitioners (Table 5.1). Although 94.4\% of the midwives provided a total midwifery service, over half of the respondents $(58.5 \%)$ also worked in a shared care model with doctors, either obstetricians or general practitioners, and $12 \%$ also provided part-care only, e.g. postnatal care or intrapartum care. For $90 \%$ of the respondents however, LMC midwifery care was their predominant model of midwifery. Only $5.5 \%$ of the respondents indicated that shared care with doctors was their predominant model of care.

On average, the caseload of these midwives was 45.6 women per year $(95 \% \mathrm{CI}$ 42.2,49.0). They had an average individual referral rate of $35.2 \%$ (95\% CI 33.0,37.4). The referral rate of the population as a whole was also calculated using the total number of women that this group of midwives referred as the numerator and the total number of women they cared for as the denominator. This resulted in a referral rate of $34.7 \%$, which matched the individually calculated mean rate of $35.2 \%$

\section{Location of the midwives}

In order to ascertain the location and regional spread of the respondents, they were asked to provide the name of the secondary care maternity facility they most commonly used. Overall, $74.8 \%$ of midwives were located in the North Island and $25.2 \%$ in the South Island. Each hospital in New Zealand was represented and the spread reflected the locations of the entire childbearing population (Table 5.2). Three quarters of the referrals were made to hospitals in the North Island with the biggest concentration (20.9\%) in the Auckland region. 
Table 5.2 Location of referring hospital most frequently used by midwives, compared with national distributions of births by hospital.

\begin{tabular}{|c|c|c|c|c|}
\hline $\begin{array}{l}\text { Regions of New } \\
\text { Zealand }\end{array}$ & $\begin{array}{l}\text { Midwives } \\
\text { by region. } \\
\text { n(\%) }\end{array}$ & $\begin{array}{l}\text { Secondary maternity } \\
\text { hospitals by region }\end{array}$ & $\begin{array}{l}\text { Midwives by } \\
\text { hospital. } \\
\text { n(\%) }\end{array}$ & $\begin{array}{l}\text { Births in } \\
\text { hospitals* } \\
(\mathrm{n} \%)\end{array}$ \\
\hline \multicolumn{5}{|l|}{ North Island } \\
\hline Northland & $16(5.2)$ & Northland & $16(5.2)$ & $1340(2.8)$ \\
\hline \multirow[t]{5}{*}{ Auckland } & $65(20.9)$ & & & \\
\hline & & North Shore & $14(4.4)$ & $2979(6.3)$ \\
\hline & & Waitakere & $10(3.2)$ & $2521(5.3)$ \\
\hline & & National Women's** & $22(7.1)$ & $7892(16.8)$ \\
\hline & & Middlemore** & $19(6.1)$ & $3711(7.9)$ \\
\hline Waikato & $27(8.7)$ & Waikato** & $27(8.7)$ & $2996(6.4)$ \\
\hline \multirow{3}{*}{ Bay of Plenty } & $14(4.5)$ & & & \\
\hline & & Tauranga & $10(3.2)$ & $1851(3.9)$ \\
\hline & & Whakatane & $4(1.3)$ & $716(1.5)$ \\
\hline \multirow[t]{3}{*}{ East Coast } & $15(4.9)$ & & & \\
\hline & & Gisborne & $7(2.3)$ & $723(1.5)$ \\
\hline & & Hastings & $8(2.6)$ & $2090(4.4)$ \\
\hline Rotorua & $11(3.5)$ & Rotorua & $11(3.5)$ & $1368(2.9)$ \\
\hline New Plymouth & $11(3.5)$ & Taranaki Base & $11(3.5)$ & $1191(2.5)$ \\
\hline Wanganui & $11(3.5)$ & Wanganui & $11(3.5)$ & $737(1.6)$ \\
\hline Palmerston North & $22(7.1)$ & Palmerston North & $22(7.1)$ & $1838(3.9)$ \\
\hline \multirow[t]{4}{*}{ Wellington } & $40(13)$ & & & \\
\hline & & Wgtn Women's** & $29(9.4)$ & $3346(7.1)$ \\
\hline & & Hutt Valley & $8(2.6)$ & $1994(2.5)$ \\
\hline & & Masterton & $3(1)$ & 461(.9) \\
\hline Total Nth Island & $232(74.8)$ & & & 37754(80.2) \\
\hline \multicolumn{5}{|l|}{ South Island } \\
\hline Blenheim & $4(1.3)$ & Wairau & $4(1.3)$ & $467(1)$ \\
\hline Nelson & $10(3.2)$ & Nelson & $10(3.2)$ & $889(1.9)$ \\
\hline Christchurch & $35(1.3)$ & Chch Women's** & $35(1.3)$ & $4165(8.8)$ \\
\hline Greymouth & $5(1.6)$ & Greymouth & $5(1.6)$ & $283(.6)$ \\
\hline Timaru & $3(1)$ & Timaru & $3(1)$ & $585(1.3)$ \\
\hline Dunedin & $16(5.2)$ & Dunedin** & $16(5.2)$ & $1770(3.8)$ \\
\hline Southland & $5(1.6)$ & Southland & $5(1.6)$ & $1153(2.4)$ \\
\hline Total Sth Island & $78(25.2)$ & & & $9312(19.8)$ \\
\hline National Total & $310(100)$ & & & $47066(100)$ \\
\hline
\end{tabular}

There were marked differences in the distance that midwives lived from a secondary care facility. Although $70 \%$ of the midwives lived less that $25 \mathrm{kms}$ from the hospital, there were 12 midwives who lived over $100 \mathrm{kms}$ from the hospital and one who lived 250 kms away. 


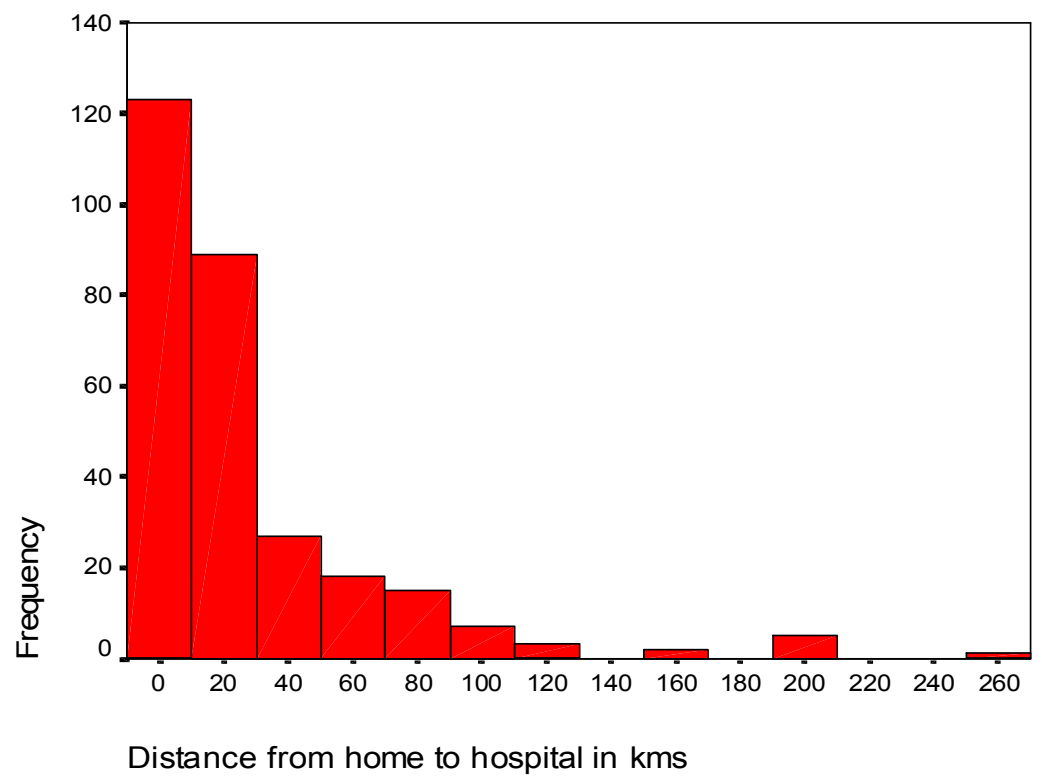

Figure 5.1 Distance from midwives' homes to secondary care hospital

Although the mean distance was $27 \mathrm{kms}$, the median was $12 \mathrm{kms}$ and the mode was 5kms. There were also significant outliers (SD 38.3, Range 1-250, variance 1467.9, 95\% CI 22.6, 31.4). For these reasons, this data could not be used as a continuous variable but was transformed into two groups. The two groups represented those who had easier access to the secondary care facility and those who were at a greater distance away. The distance chosen as the dividing line for the two groups was $40 \mathrm{kms}$, as this distance represented both one hour's transfer time and was, in several circumstances, the distance from level one maternity units to the secondary maternity service. The groups were named as 'urban' and 'rural' although it must be noted that some of the midwives in the rural group were living in New Zealand towns without a secondary maternity service rather than in rural country areas.

\section{The referred women}

The midwives were asked to provide detail on all the women in their care who had a referral for obstetric consultation during the four-month period of the study. Thirty eight midwives did not refer any of their women for a referral for obstetric consultation during the period of the study. Twenty-five midwives, who between them had 66 clients, did not report the details of their referrals. 


\section{Demographic detail of the referred women}

The questionnaire asked the midwives to provide the demographic and referral data of the referred women (Table 5.3).

Table 5.3 Demographic and birth outcome data of women referred for obstetric consultation.

\begin{tabular}{lccllll}
\hline & $\mathrm{N}^{*}$ & $\%$ & Mean & SD & Range & $95 \%$ CI \\
\hline Age & 1387 & 99.4 & 28.8 & 6.0 & $14-46$ & $28.5,29.1$ \\
Ethnicity & 1396 & 100.0 & & & & \\
European & 1012 & 71.7 & & & $69.3,74.1$ \\
Maori & 236 & 16.7 & & & $14.7,18.7$ \\
Pacific & 70 & 5.0 & & & $3.8,6.2$ \\
Asian & 56 & 4.0 & & & 3,5 \\
Other & 22 & 1.6 & & & & $0.9,3.3$ \\
Parity & 1390 & 100.0 & & & \\
0 & 474 & 34.1 & & & $31.6,36.6$ \\
1 & 466 & 33.5 & & & 31,36 \\
2 & 270 & 19.4 & & & $17.3,21.5$ \\
3 & 98 & 7.1 & & & $5.7,8.5$ \\
$>3$ & 82 & 5.9 & & & $4.6,7.2$ \\
& & & & & \\
Birth Outcomes & 1359 & 100.0 & & & & \\
Normal birth & 689 & 50.7 & & & & $48,53.4$ \\
Forceps or ventouse & 180 & 13.2 & & & & $33.4,15$ \\
Caesarean section & 489 & 36.0 & & & & $24.3,29.1$ \\
Epidural & 363 & 26.7 & & & $1.2,2.6$ \\
Perinatal death & 26 & 1.9 & & \\
\hline
\end{tabular}

The ages of the referred women ranged from 14 to 46 years. Most were European but $16.7 \%$ were Maori. Approximately one-third of the women were having their first baby and one-third, their second. A half of the referred women had a normal birth, and $36 \%$ had a caesarean section. The normal birth rate for all the women cared for by the midwives, including those not referred was $81 \%(3466 / 4251)$ and the caesarean section rate was $11.5 \%(489 / 4251)$.

\section{Referral for obstetric consultation}

The survey elicited detailed information on the referral processes of 1,408 women. Of these women, two thirds were referred in only one episode of the childbearing process and a third in two or more episodes. Two and a half percent were referred for obstetric consultation in all three episodes (Table 5.4). 
Table 5.4 Numbers of women referred according to childbearing episode

\begin{tabular}{lrl}
\hline $\begin{array}{l}\text { Childbearing episodes in } \\
\text { which women were referred }\end{array}$ & Number & \% (95\%CI) \\
\hline Antenatal only & 516 & $36.6 \%(43,39.2)$ \\
Intrapartum only & 383 & $27.3 \%(24.5,30.1)$ \\
Postpartum only & 24 & $1.5 \%(0.9,2.1)$ \\
Antenatal and intrapartum & 411 & $29.4 \%(27,31.8)$ \\
Antenatal and postpartum & 23 & $1.5 \%(0.9,22.1)$ \\
Intrapartum and postpartum & 17 & $1.2 \%(06,1.8)$ \\
All three episodes & 32 & $2.5 \%(1.7,3.3)$ \\
\hline
\end{tabular}

Total

1408

The greatest proportion of referrals in one pregnancy period was in the antenatal period $(36.6 \%)$. Women who had referrals in more than one period had them mostly in the antenatal and intrapartum periods $(29.4 \%)$.

\section{Antenatal referral}

Most antenatal referrals were made to a known obstetrician at the hospital, $30 \%$ were made to a hospital team which might have included registrars and house surgeons (Table 5.5). A small number were made to a private obstetrician where payment by the women to the obstetrician would have been required. The midwife accompanied the woman to the first consultation in $40 \%$ of the cases. This was most likely to happen where the visit was to an obstetric team, rather than to a known obstetrician at the hospital, and least likely where the visit was to a private obstetrician $\left(\chi^{2} 28.66\right.$, df $2, \mathrm{p}=.000)$

Table 5.5 Practitioners to whom antenatal referrals were made

\begin{tabular}{lrll}
\hline & N & $\begin{array}{l}\text { \% of total } \\
\text { referrals }\end{array}$ & $\begin{array}{l}\% \text { of antenatal } \\
\text { referrals }\end{array}$ \\
\hline Antenatal referral & 982 & $69.6 \%$ & \\
Referral made to: & & & \\
$\quad$ Hospital team/clinic & 297 & & $30.9 \%$ \\
$\quad$ Obstetrician-private & 68 & $7.1 \%$ \\
$\quad$ Obstetrician-public & 595 & $62.0 \%$ \\
Missing data & 22 & \\
Midwife present at & 378 & & \\
first consultation & & & $39.6 \%$ \\
\hline
\end{tabular}

The reasons for antenatal referrals were numerous and are detailed in Figure 5.2. The question of 'reason for referral' was a multiple response question. The midwives were asked to provide as many reasons as they felt were appropriate. In the antenatal period no reason for referral was provided in 20 cases and thus was classified as 
missing data. Ten of the referrals had two reasons and in six cases of referral there were three reasons provided.

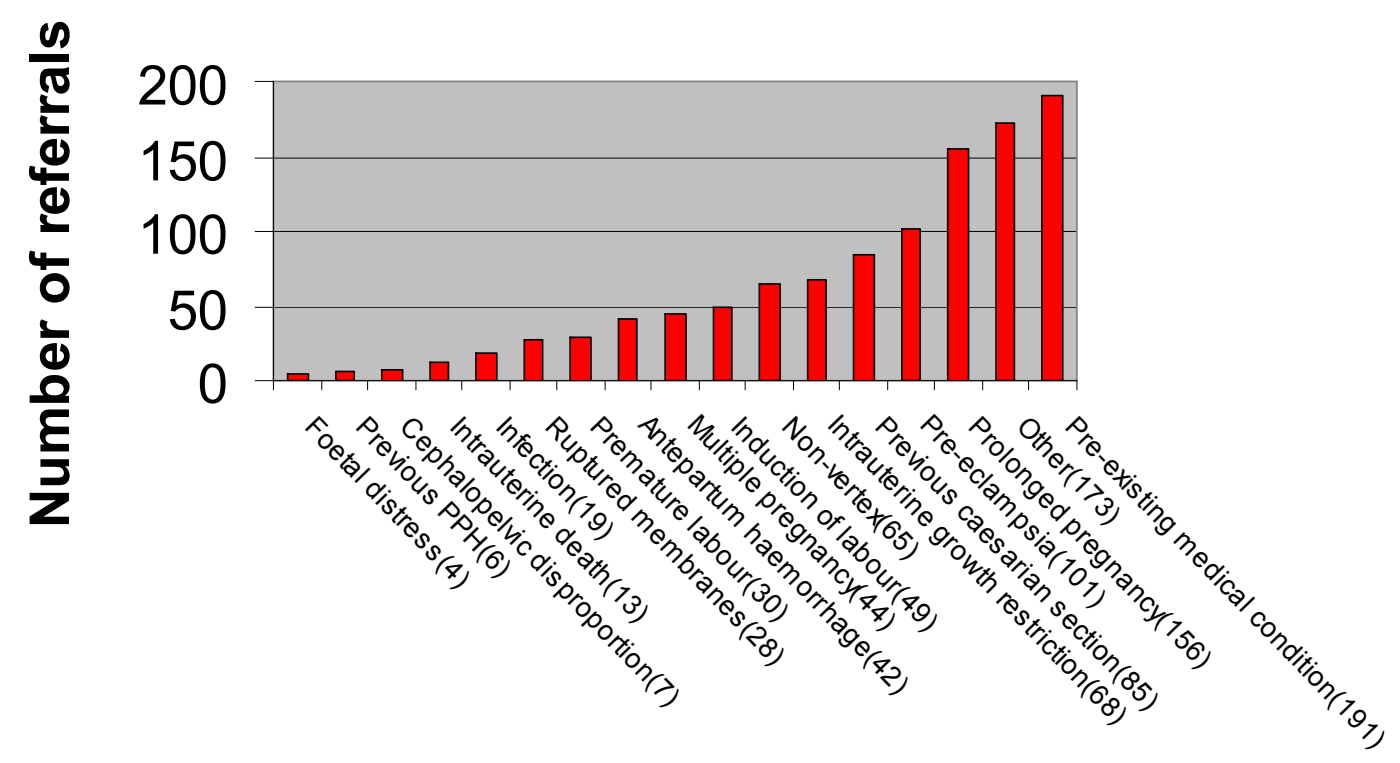

\section{Reasons for referral}

Figure 5.2 Reasons for antenatal referrals

The most common reasons for antenatal referral were pre-existing medical conditions and 'other'. There were also many referrals for prolonged pregnancy. As prolonged pregnancy is of particular interest in its relationship with risk management it was explored further. Of the 156 women who were referred for prolonged pregnancy, $23 \%(36 / 156)$ were delivered by caesarean section and $11 \%(17 / 156)$ had either a forceps or ventouse delivery. Only 5\% (8/156) of the women had any degree of co morbidity. Three were also referred for pre-eclampsia, one for pre-existing medical condition, one for intrauterine growth restriction and three for 'other'. Twenty nine $(18.6 \%)$ of the women who were referred for prolonged pregnancy were also referred in the intrapartum period for prolonged labour.

As expected there were a large number of referrals for 'other', a fact common to most databases which include reasons for obstetric referral or admission (MacVicar et al., 1993; New Zealand Health Information Service, 2003; Wallace et al., 1995). The category 'other' is the accumulation of a large number of conditions each of which 
will occur very infrequently. There were also a large number of referrals for 'preexisting medical condition'.

\section{Intrapartum referral}

The same questions were asked about intrapartum referrals (Table 5.6). Most referrals were made directly to a known obstetrician employed in the hospital, followed by referrals to the hospital team. The team includes obstetricians and registrars who work on a rotating roster system. When a team system is operating the women will often be seen by a registrar. Only 6\% (53/846) of intrapartum referrals were made to a private obstetrician, not necessarily 'on call' via the hospital system.

Table 5.6 Practitioners to whom intrapartum referrals were made

\begin{tabular}{lrcc}
\hline & $\mathrm{N}$ & $\%$ of total referrals & $\%$ of intrapartum referrals \\
\hline Total referrals & $1395^{*}$ & & \\
Intrapartum referral & 847 & $60.6 \%$ & \\
Referral made to: & & & $35.7 \%$ \\
Hospital team/clinic & 291 & & $6.5 \%$ \\
Obstetrician-private & 53 & $57.8 \%$ \\
Obstetrician-public & 472 & \\
Missing data & 30 & & \\
\hline
\end{tabular}

* This number is smaller than the 1408 total referrals as there was missing data in 13 cases

As with the antenatal referrals 'reason for referral' was a multiple response question. In the intrapartum period, no reason for referral was provided in 23 cases and thus was classified as missing data. Twenty eight of the referrals were for two reasons and in 15 cases there were three reasons for referral.

The reasons for referral were numerous and there were, as in the antenatal period, a large number of referrals for 'other' (Figure 5.3). There were also a large number of referrals for foetal distress. Most intrapartum referrals were made for lack of progress. 


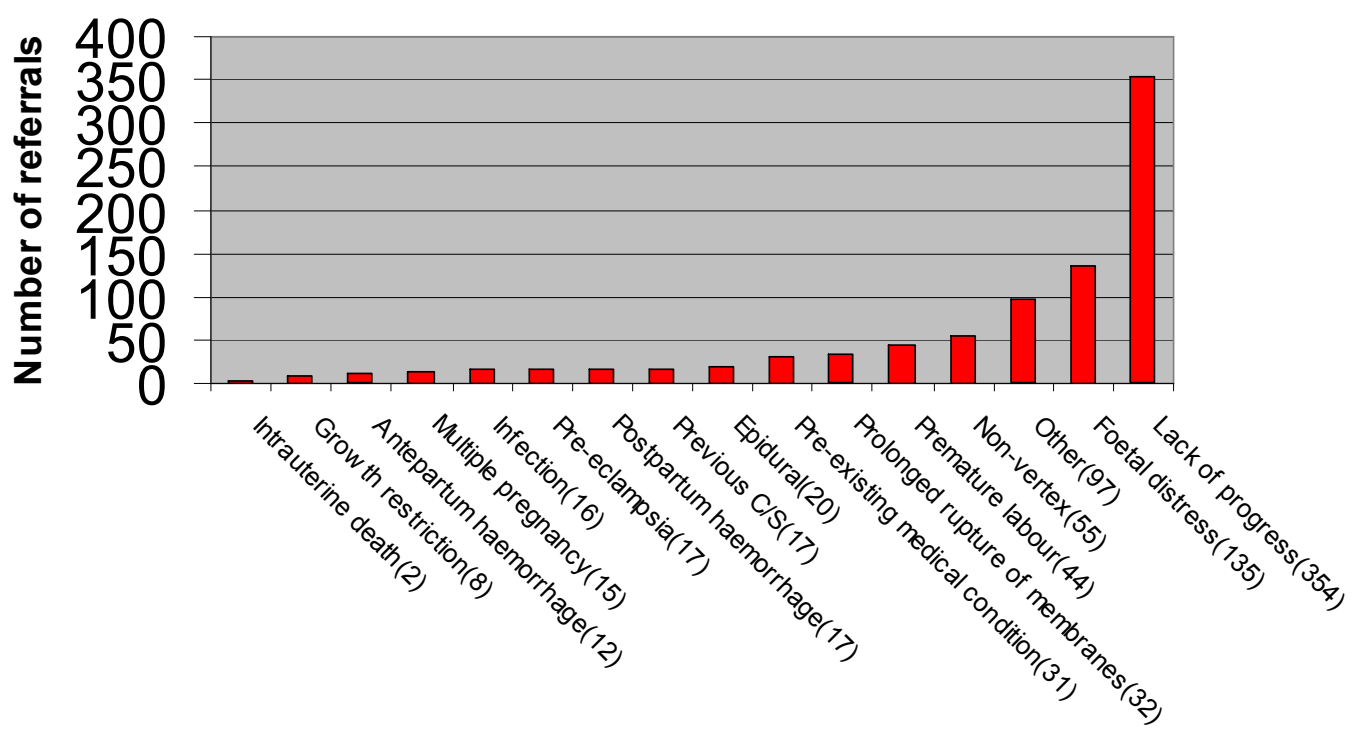

Reasons for referral

Figure 5.3 Reasons for intrapartum referrals

As in the case of 'prolonged pregnancy' in the antenatal period, 'lack of progress' is of particular interest in the intrapartum period. Of the women who had a referral for lack of progress, 48\% (169/345) had a caesarean section and 30\% (105/345) had either a forceps or ventouse delivery. Only 13\% (45/345) had any degree of co morbidity recorded. One had an infection and one had premature rupture of the membranes. Three were in premature labour and 5 babies were be non-vertex presentations. Ten women had cephalo-pelvic disproportion and seven were 'other'. Eighteen also had foetal distress.

\section{Postpartum referral}

The number of postpartum referrals was considerably smaller (Table 5.7). The proportion of referrals to the hospital team at $43 \%$ was larger than in the previous phases. 
Table 5.7 Practitioners to whom postpartum referrals were made

\begin{tabular}{lrlr}
\hline & $\mathrm{N}$ & $\begin{array}{l}\% \text { of total } \\
\text { referrals }\end{array}$ & $\begin{array}{l}\text { \% of postpartum } \\
\text { referrals }\end{array}$ \\
\hline Total referrals & 1408 & & \\
Postpartum referral & 96 & $6.9 \%$ & \\
Referral made to: & & & $42.9 \%$ \\
$\quad$ Hospital team/clinic & 39 & & $6.6 \%$ \\
$\quad$ Obstetrician-private & 6 & $50.5 \%$ \\
$\quad$ Obstetrician-public & 46 & \\
Missing data & 5 & & \\
\hline
\end{tabular}

The most common reasons for postpartum referral were infection and postpartum haemorrhage and there were again a high number of referrals for 'other' (Figure 5.4). In only 2 cases was more than one reason for postpartum referral provided. There were missing data in 2 cases.

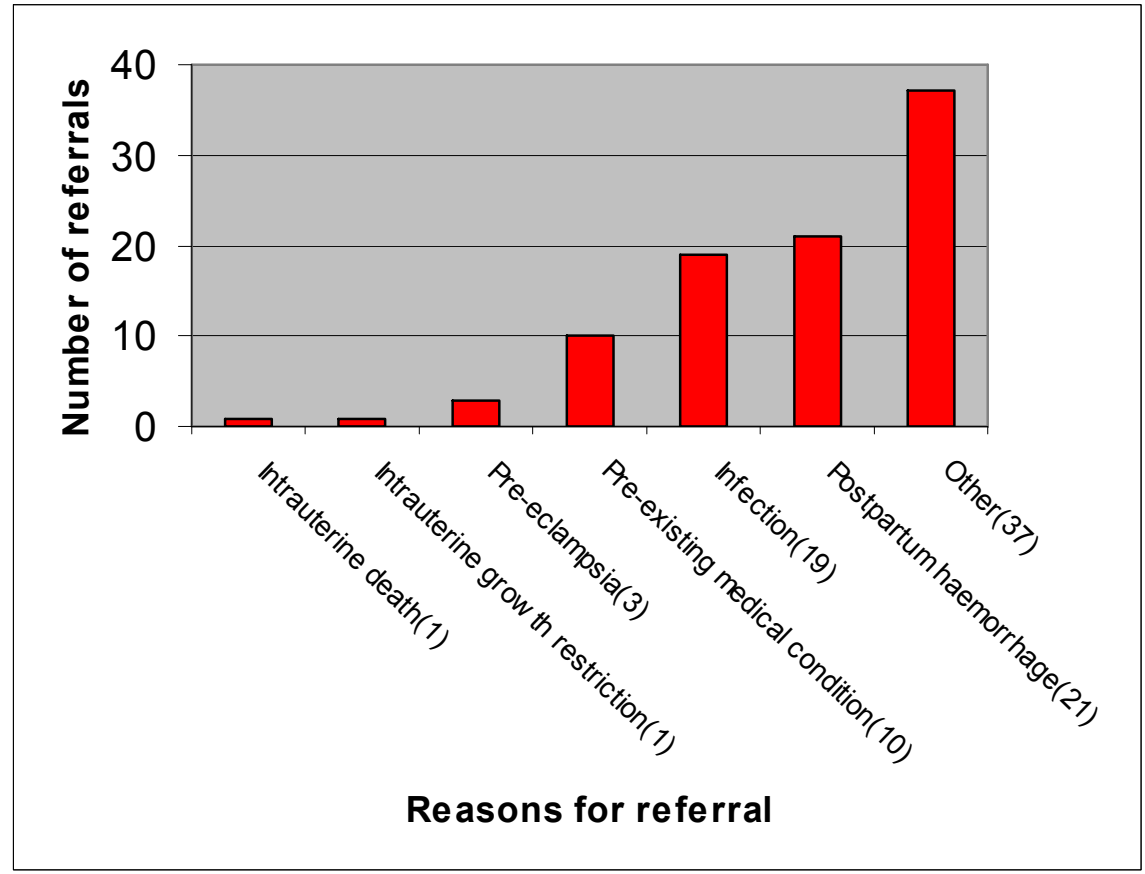

Figure 5.4 Reasons for postpartum referrals

\section{Transfer of clinical responsibility and LMC}

Information on 1,408 of the 1,477 women who had a referral for obstetric consultation was provided. Of the 1,408 women who were referred, 608 (43\%) were considered to have had a transfer of clinical responsibility (Figure 5.5). The remaining women had 
a consultation only. Of these 608 transfers, 326 (54\%) had LMC signed over to the secondary care service.

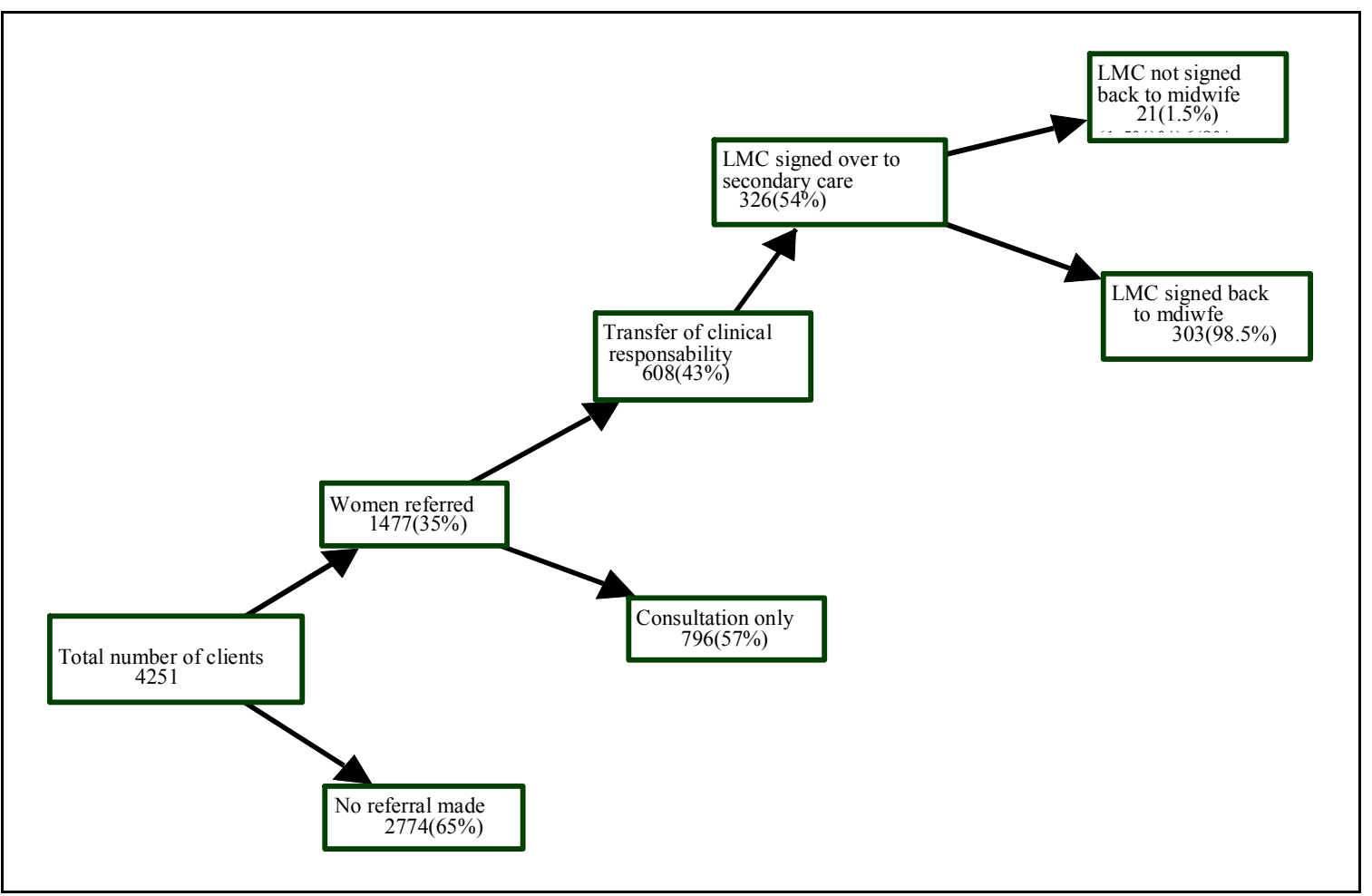

Figure 5.5 Referral and transfer of clinical responsibility

(Data are missing from some fields so totals do not equate. See Tables 5.9 and 5.10 for details of missing data.)

Once transfer of clinical responsibility had occurred, $74 \%(415 / 562)$ of the women in the study continued care with the midwife (Figure 5.6). In $26 \%(108 / 409)$ of cases the midwives who continued care received no payment. This was more likely to happen where there had been transfer of responsibility or sign-over of LMC in the antenatal rather than in the intrapartum period. (n 430, $\chi^{2} 14.88 \mathrm{df} 2, \mathrm{p}=.001$ ).

One hundred and forty nine women had no ongoing care with their midwife. This represents $10.4 \%(147 / 1408)$ of all the women who had referrals for obstetric consultation and 3.5\% (147/4251) of the total caseload of the midwives. Ninety six percent of women therefore maintained contact with, and received care from their original LMC midwife through the childbearing process, regardless of their level of risk. 


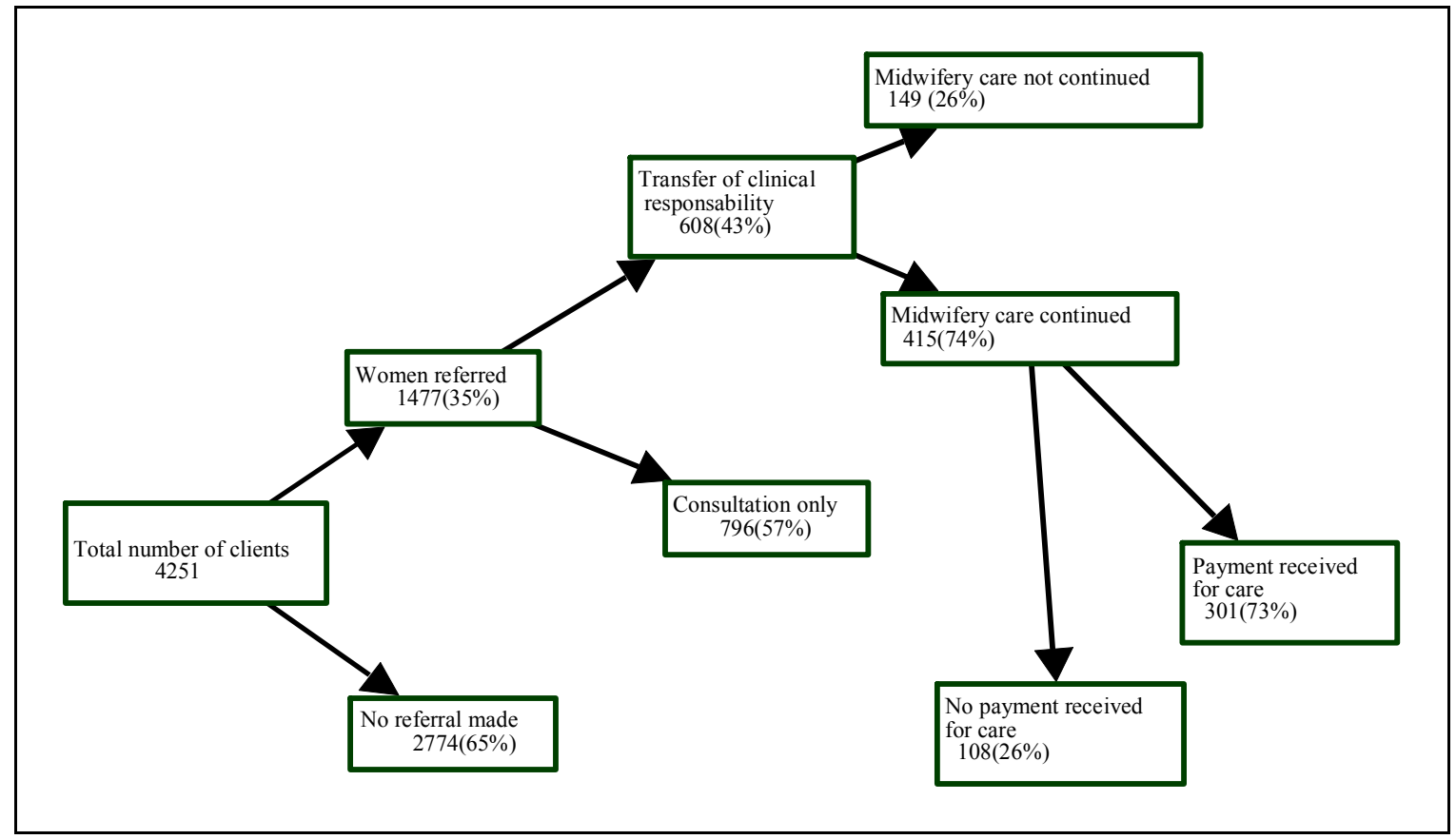

Figure 5.6 Continuation of midwifery care once clinical responsibility transferred (Data are missing from some fields so totals do not equate.

See Table 5.9 and 5.10 for details of missing data.)

The information was further analysed to describe these practices according to the antenatal, intrapartum and postpartum episodes of care (Figure 5.7). Thirty five percent of the women had referrals during more than one pregnancy episode. Thus the number of referrals, broken down by pregnancy episode, is greater than the total number of referrals. 


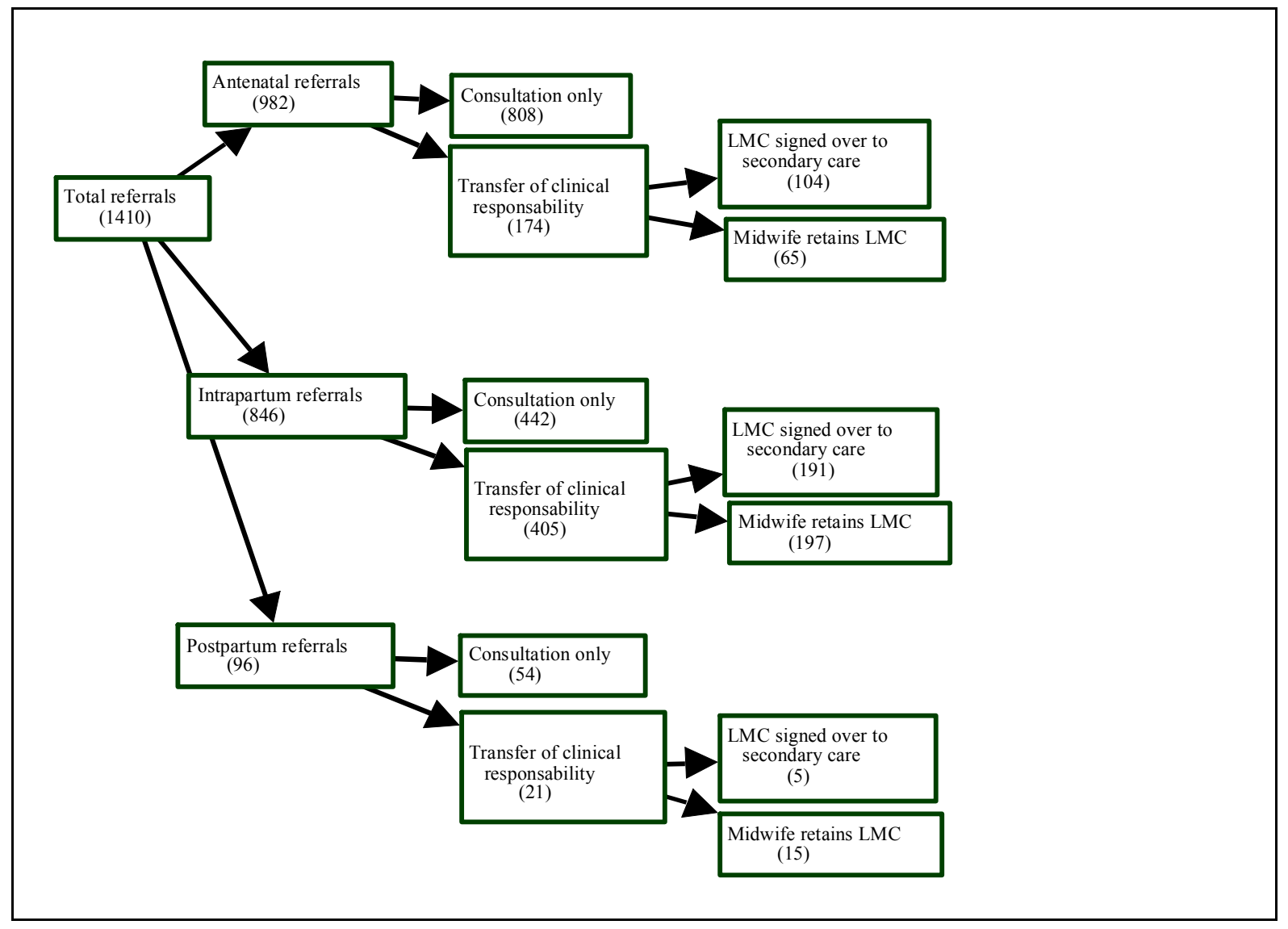

Figure 5.7 Referral and transfer of clinical responsibility by childbearing period (Data are missing from some fields so totals do not equate.

See Table 5.9 and 5.10 for details of missing data.)

The majority $(82 \%)$ of the antenatal consultations did not result in any transfer of clinical responsibility as contrasted with the intrapartum consultations where $52 \%$ involved consultation only. Once clinical responsibility had been transferred it was more likely that LMC was signed over in the antenatal period than in the intrapartum period. LMC was usually signed back to the midwives, most commonly in the postnatal period. There were only 21 instances recorded where LMC was not signed back to the midwife at any stage.

Once transfer of clinical responsibility of care had occurred, women were less likely to receive ongoing care from their midwife if this transfer had occurred in the antenatal period $(35.6 \%)$ than in the intrapartum period $(19.7 \%)$. During the postpartum period, only 21 women $(21.8 \%)$ who required a referral had a transfer of clinical responsibility. Eighty two percent had midwifery care continued. 
Midwives were most likely to provide care for no payment where clinical responsibility had been transferred in the postnatal period (50\%). This was followed by the antenatal period (35.8\%) and the intrapartum period $(23.5 \%)$.

For clarity of presentation, missing data are presented separately in Tables 5.8 and 5.9. The questionnaire requested a large amount of detailed information on each of the midwives' clients. As this section of the study asked questions that progressively reduced the variables, the missing data are cumulative. As each question was asked, missing data from the previous question were reflected in the following question. The most missing data were in the section relating to the continuation of midwifery care (Table 5.9).

Table 5.8 Missing data where transfer of clinical responsibility is described

\begin{tabular}{|c|c|c|c|c|c|}
\hline Total referrals & 1408 & 1408 & 1408 & 1408 & 1408 \\
\hline Consultation only & 796 & 796 & 796 & 796 & 796 \\
\hline Transfer of clinical responsibility & 608 & & & & \\
\hline Stage of transfer* & & AN 174 & & & \\
\hline $\mathrm{AN} / \mathrm{IP} / \mathrm{PP}$ & & $\begin{array}{r}\text { IP } 405 \\
\text { PP } 21\end{array}$ & & & \\
\hline Midwife retains LMC & & & 267 & 267 & 267 \\
\hline LMC signed over to secondary care & & & 326 & & \\
\hline LMC not signed back to midwife & & & & 21 & 21 \\
\hline LMC signed back to midwife & & & & 303 & \\
\hline \multirow[t]{3}{*}{ Stage LMC signed back AN/IP/PP* } & & & & & AN 4 \\
\hline & & & & & IP 13 \\
\hline & & & & & PP 283 \\
\hline Missing data** & 4 & 12 & 19 & 21 & 24 \\
\hline
\end{tabular}

*AN(antenatal) IP(intrapartum) PP(postpartum)

**Cumulative 
Table 5.9 Missing data where midwifery care continued after clinical responsibility handed over

\begin{tabular}{lrrr}
\hline Total referrals & 1408 & 1408 & 1408 \\
\hline Consultation only & 796 & 796 & 796 \\
Transfer of clinical responsibility & 608 & & \\
Midwifery care not continued & & 147 & 147 \\
Midwifery care continued & & 415 & \\
No payment received for continued care & & & 108 \\
Payment received for continued care & & & 301 \\
\hline Missing data* & 4 & 50 & 56 \\
\hline Cumulative & & &
\end{tabular}

There were 26 instances of missing data in the transfer of clinical responsibility and handover of LMC data, and 58 in the continued midwifery care data.

\section{Birth outcome}

Of those women who were referred, 50.7\% (689/1359) had a normal (unassisted) vaginal birth, 13.2\% (180/1359) had an assisted vaginal birth, either with forceps or ventouse, and 36\% (488/1359) had a caesarean section. When extrapolated to the whole population of those women cared for by the midwives, the normal birth rate was $83.8 \%(3463 / 4132)$, the forceps/ventouse rate was $4.4 \%(180 / 4132)$ and the caesarean section rate was $11.8 \%$ (489/4132). Given that the caseload of each midwife was known, it was possible to calculate the caesarean rate as a continuous variable. The midwives' average caesarean section rate was $12.7 \%$ (N 238, SD 11.6, 95\% CI 11.2, 14.2). The caesarean section rate of the total population of 1408 referred women had missing data in 119 of cases.

The data on epidural anaesthesia and perinatal mortality were not analysed further as the design of the questionnaire precluded any assessment of missing data. 


\section{Section Two: Attitudes of the midwives}

\section{Responses to attitudinal statements}

The survey asked the midwives to agree or disagree with nine Likert-scale attitudinal statements. These statements related to the pattern of referral, the extent of collaboration and support with the secondary maternity service, and the medico-legal environment. There was strong support for independent midwives to care for both low-risk and high-risk women. Seventy percent of the midwives disagreed with the statement that independent midwives should care for only low risk women (Figure 5.8). Thirteen percent neither agreed nor disagreed.

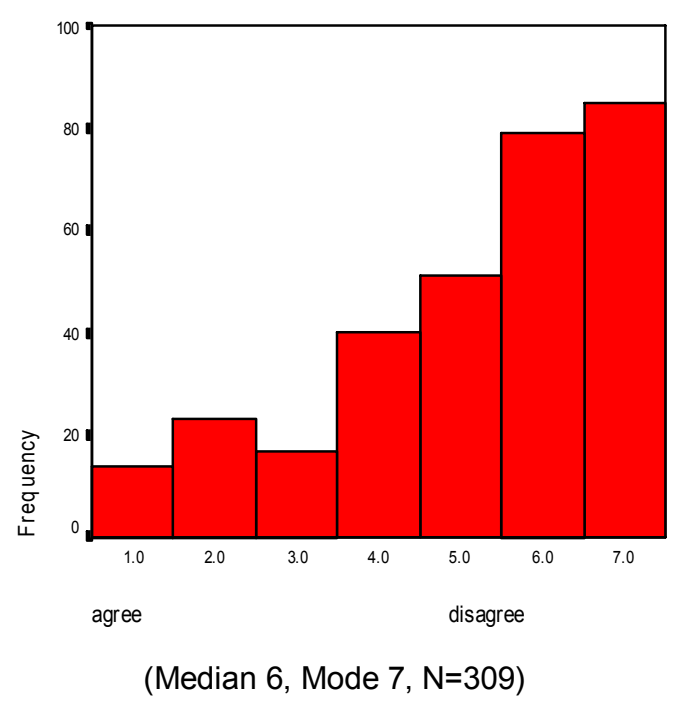

Figure 5.8 Independent midwives should care only for low risk women

There was strong agreement that risk management was an important part of their practice. Eighty eight percent of midwives agreed to some extent with this statement. Forty one percent agreed strongly. 


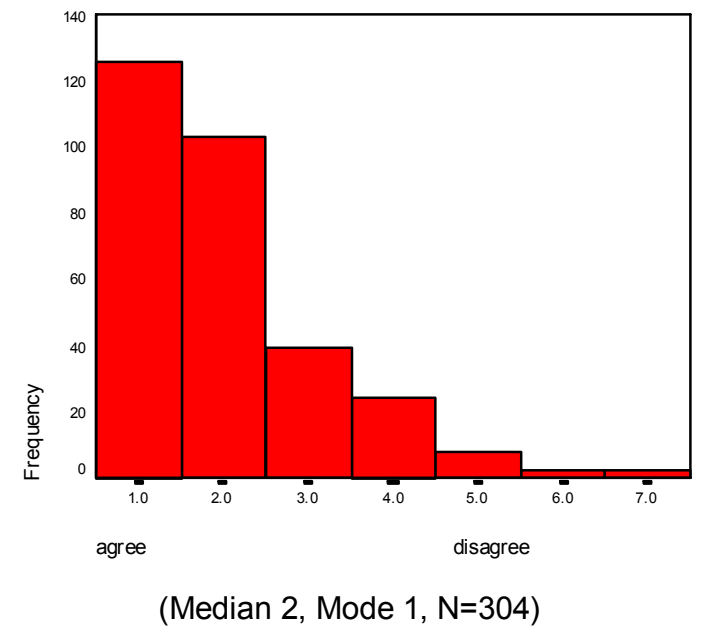

Figure 5.9 Risk management is an important part of my practice

The midwives were asked about the referral processes. Although most agreed to some extent that their pattern of referral was appropriate to the needs of the women they cared for (94\%) (Figure 5.10), and that the referral guidelines were a useful tool (81\%) (Figure 5.11), they were more likely to be undecided $(23 \%)$, or to think that the guidelines required them to refer more often than was necessary(46\%) (Figure 5.12).

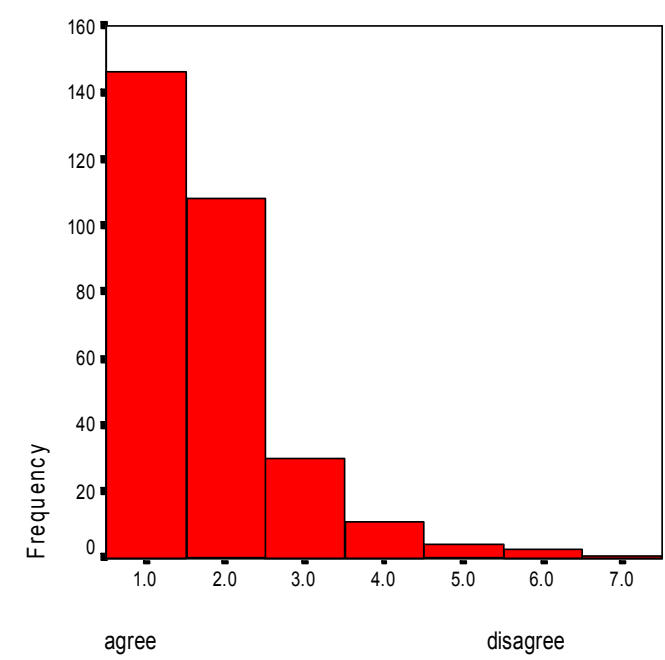

(Median 2, Mode 1, N=303)

Figure 5.10 My pattern of referral is appropriate to the needs of the women I care for

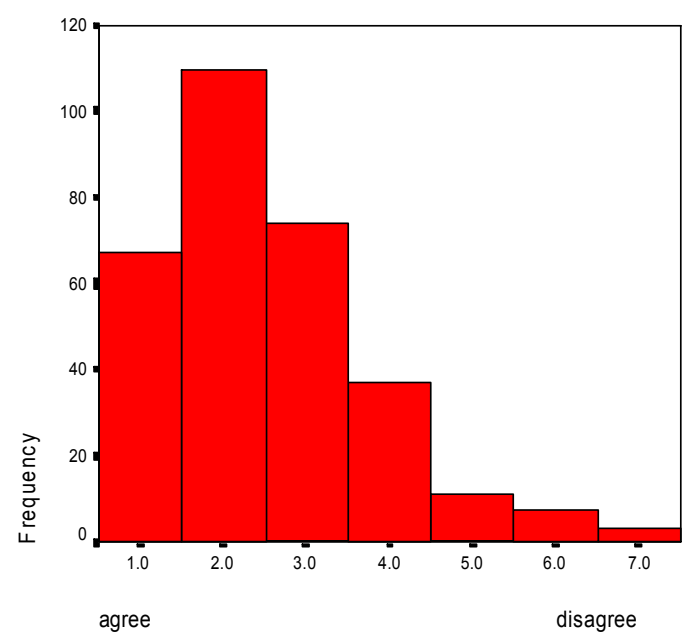

(Median 2, Mode 2, N=309)

Figure 5.11 The referral guidelines are a useful tool when I consider the need for referral 


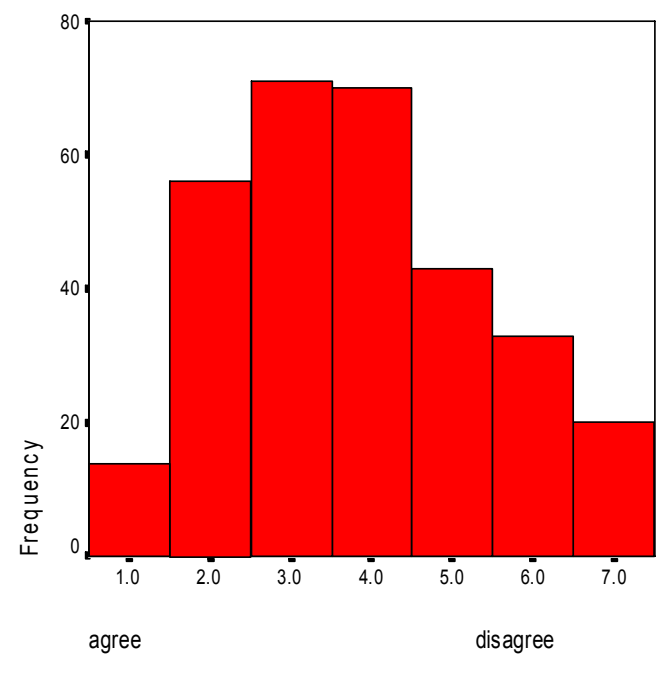

(Median 4, Mode 3, N=307)

Figure 5.12 The referral guidelines require me to refer more often than is necessary

The majority of midwives agreed to some extent that there was both excellent collaboration between primary and secondary care (64\%) (Figure 5.13) and that obstetricians supported continuity of midwifery care (72\%) (Figure 5.14). Twenty four percent of the midwives did not think that there was excellent collaboration in their regions and $14 \%$ did not think their obstetricians were supportive of continuity of midwifery care.

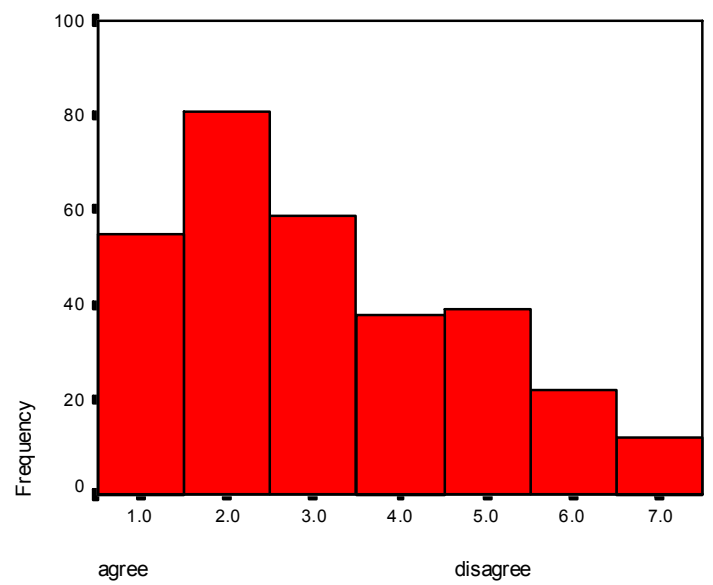

(Median 3, Mode 2, N=306)

Figure 5.13 In my region there is excellent collaboration between primary and secondary care

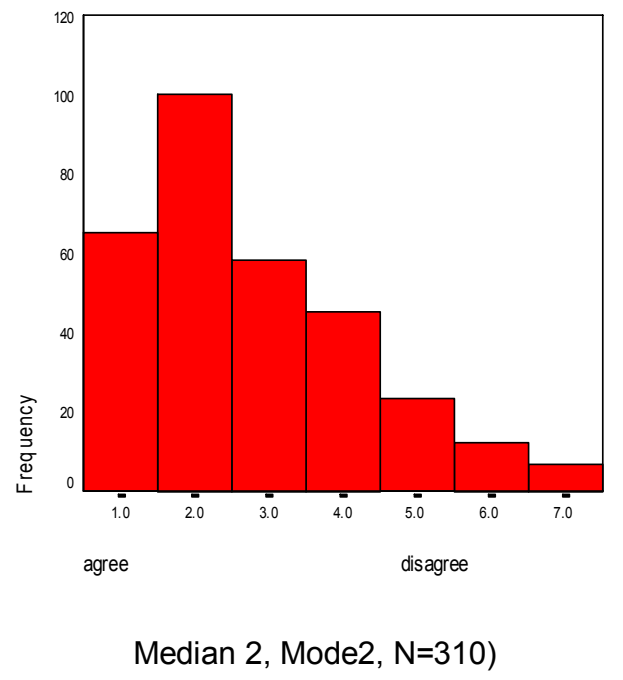

Figure 5.14 Obstetricians in my area in general support continuity of midwifery care 
There was a tendency to disagree that the medico-legal environment made childbirth safer for women (Figure 5.15). Fifty five percent disagreed with this statement and $20 \%$ neither agreed nor disagreed. There was a corresponding number of midwives who agreed that the medico-legal environment restricted their practice (Figure 5.16). Forty nine percent agreed and twenty three percent neither agreed nor disagreed.

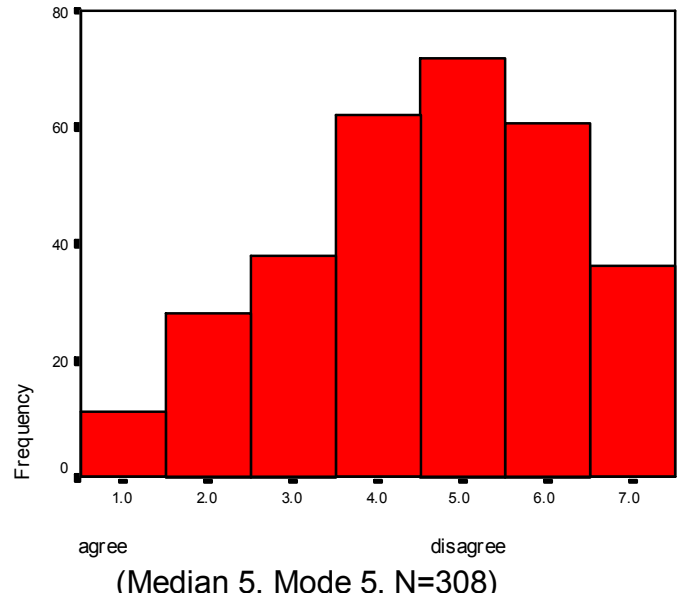

(Median 5, Mode 5, $\mathrm{N}=308$ )

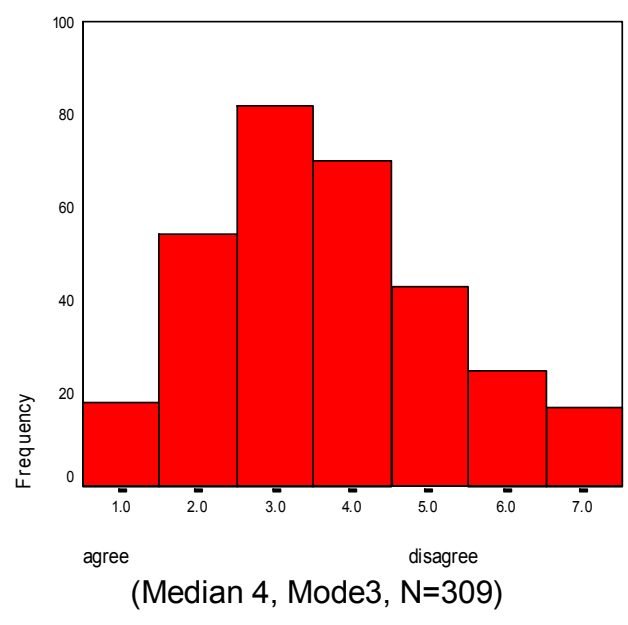

Figure 5.16 The medico-legal environment restricts me in my practice.

Principle factor analysis revealed one factor which could be used in further statistical analysis. This was identified as 'a supportive primary/secondary interface' (Figure 5.17) and in subsequent analysis replaces the two items: 'In my region there is excellent collaboration between primary and secondary maternity services' and 'Obstetricians in my regions in general support continuity of maternity care' 


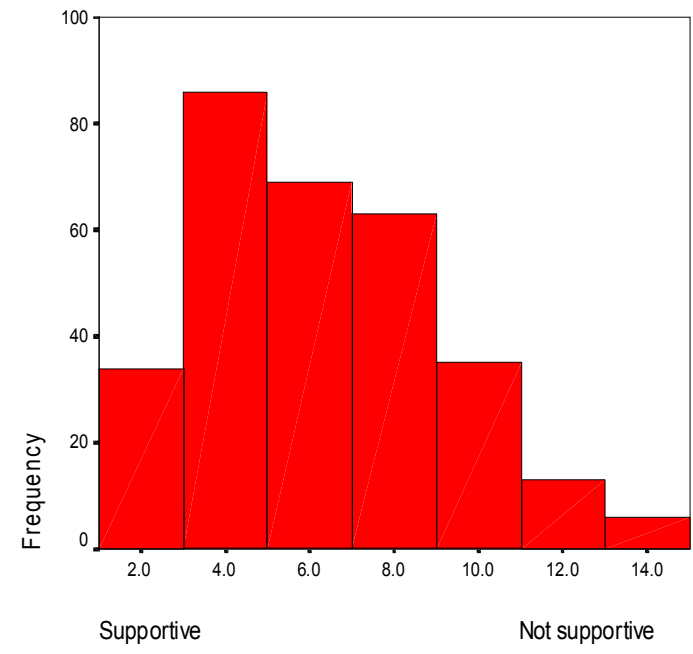

(Median 5, Mode 4, N=306)

Figure 5.17 A supportive and collaborative primary/secondary interface

\section{Section Three: Relationships between referral and the demographic and attitudinal characteristics of the midwives.}

This study seeks to ascertain both what the practice and attitudes of New Zealand LMC midwives are in regard to risk and also to investigate what might impact on these practices and attitudes. Both referral for obstetric consultation and caesarean section are important markers and as such it was of interest to explore what factors might impinge on these outcomes.

\section{The referral rate}

The only association between referral rate and any of the demographic and attitudinal variables in the study was with caseload $(n=247 \mathrm{r}=-.142 \mathrm{p}=.026)$. A higher caseload was associated with a lower referral rate. Although there was a statistically significant association between caseload and years of experience $(n=269 . r=.357$, $\mathrm{p}=.000$ ), the association between experience and referral rate was not statistically significant $(\mathrm{n}=238, \mathrm{r}=-.081 \mathrm{p}=.214)$. Further exploration of experience included regrouping the variable into those with five years and less experience and those with three years and less experience as it was thought that it may be that midwives new to practice had higher referral rates. No significant differences were found. The referral rate was positively correlated with the caesarean section rate $\left(n=247, r_{2}=.318\right.$ $\mathrm{p}=.000)$. 
There was no relationship found between referral rate and age, country of registration, being a nurse or direct entry midwife, whether the midwives were self employed or employed, or lived in rural or urban areas. It was not possible to ascertain the difference between all the regions as some had a very small number of participants. The referral rates of the four main regions - Auckland, Hamilton, Wellington and Christchurch were explored, as were the referral rates in the North and South Islands of New Zealand. No significant differences were found.

As caseload had a significant association with referral rate (the higher the caseload the lower the referral rate), this issue was further investigated to ascertain whether there were any characteristics of the midwives with a high caseload that might distinguish them from the rest of the midwifery population. In order to do this, caseload was transformed into low, medium and high numbers according to the NZCOM's recommendations. Caseload below the NZCOM recommended level represented part-time practice. An average caseload was between 30 and 60 clients per year and represented the NZCOM recommended caseload. A high caseload was defined as over 60 births per year. Midwives with the high caseload were more likely to be nurses rather than direct entry midwives $\left(\chi^{2} 11.74 \mathrm{df}=2, \mathrm{p}=.001\right)$. There was no significant difference in their attitudes. Those midwives with the highest caseload were more experienced $\left(\chi^{2}=25, \mathrm{df}=2, \mathrm{p}=.000\right)$ and had the lowest referral rate $\left(\chi^{2}=\right.$ 8.5, $\mathrm{df}=2, \mathrm{p}=.014)$. Although referral rate is associated with caesarean section rate, the group of midwives with the highest caseload (and lowest referral rates) did not have a lower caesarean rates $\left(\chi^{2}=1.5, \mathrm{df}=2, \mathrm{p}=.464\right)$. Of the four main regions, caseload was significantly higher in Auckland and the Waikato $\left(\chi^{2}=8.4, \mathrm{df}=3\right.$, $\mathrm{p}=.038$ ). This was not however reflected in their other practice patterns. Employed and self employed midwives did not vary according to caseload.

The referral rate was further explored by linear regression. Predictors included in the model were those factors which could possibly have an effect. Apart from caseload, no other variables included in the model were shown to have any effect on the rate of referral. 
Those midwives who had made referrals for prolonged pregnancy or lack of progress in labour had higher referral rates ( $\mathrm{t} 4.5, \mathrm{df} 244, \mathrm{p}=.000)$ and higher caseloads ( $\mathrm{t} 3.4$, df $277, p=.001$ ) than the other midwives in the population. They did not differ according to years of experience or attitude.

\section{The caesarean section rate}

The caesarean section rate was positively correlated with referral rate $(n=247, r 2$ $=.318 \mathrm{p}=.000$ ). The caesarean section rate was further investigated to ascertain whether it varied according to any of the other variables apart from referral rate. There was no relationship found between caesarean rate and age, experience, caseload, country of registration, having a nursing registration or being a direct entry midwife, being self employed or employed, working in rural or urban areas. As with the referral rate, it was not possible to ascertain the difference between all the regions due to the small number of participants in some regions. The four main regions Auckland, Hamilton, Wellington and Christchurch - were investigated, as were the North and South Islands. No significant difference was found in the caesarean section rates of the midwives in these regions.

There were no statistically significant relationships between the caesarean rate and being in a collaborative, supportive secondary service, or with midwives' attitudes towards the medico-legal environment, the referral guidelines or attitudes to risk management. As there was no indication of any association, further regression analysis was not undertaken.

\section{Attitudes, demographic characteristics and work patterns}

The next statistical analysis undertaken was to explore the interrelationship between the attitudinal statements, with both the demographic details and with each other. This was to ascertain what might make an impact on midwives' attitudes to risk. The demographic and work practice data were correlated with the attitudinal scores and group differences investigated, regarding the attitudes to the particular aspects of risk management made explicit in the attitudinal statements. The attitudes were correlated with each other in order to answer such questions as: Do midwives who work in a collaborative, supportive environment feel less negative about the referral guidelines 
and the impact of the medico-legal environment? Are they more or less likely to favour continuity of midwifery care for 'at risk' women?

\section{Attitudes and demographic data}

There were no statistically significant associations between any of the attitudinal statements and age, caseload or experience when compared as continuous variables. When caseload was regrouped into low, medium and high, midwives with a high caseload were more likely to agree that obstetricians in their area were supportive of continuity of midwifery care $\left(\chi^{2}=7.042 \mathrm{df}=2 \mathrm{p}=.03\right)$. There were no differences in the attitudes between direct entry midwives and midwives who had a nursing qualification. Employed midwives were more likely than self-employed midwives to consider that there was excellent collaboration in their region $(\mathrm{N}=303 \mathrm{U}=-2.97$ $\mathrm{p}=.003$ ) but there was no difference in whether they felt that obstetricians in their regions supported continuity of midwifery care. They were more likely to think that independent midwives should care for low risk women only $(\mathrm{N}=306 \mathrm{U}=-2.17$ $\mathrm{p}=.030$ ) and more likely to think that the medico-legal environment makes childbirth safer for women $(\mathrm{N}=306 \mathrm{U}=-3.26 \mathrm{p}=.001)$.

Although there was in general strong disagreement with the statement that midwives should care for low risk women only, midwives educated out of New Zealand disagreed more strongly with this $(\mathrm{N}=308 \mathrm{U}-2.94, \mathrm{p}=.003)$. Rural midwives also disagreed more strongly than urban midwives that independent midwives should care for low risk women only $(\mathrm{N}=309 \mathrm{U}=-2.19 \mathrm{p}=.028)$.

South Island midwives are more likely than North Island midwives to agree that there was excellent collaboration between the primary and secondary maternity services (M2.74, $3.26 \mathrm{U}-1.72 \mathrm{p}=.030$ ). There were no other significant differences in attitude. The attitudinal statements were also analysed according to the four main regions of New Zealand: Auckland, Waikato, Wellington and Christchurch (Table 5.10). Wellington midwives were most likely to disagree that the guidelines required referral more often than necessary, Waikato midwives most likely to disagree that midwives should care for low risk women only, and Christchurch midwives were most likely to disagree that the referral guidelines were useful when considering the need for referral. 
Table 5.10 Differences in midwives attitudes according to main New Zealand Regions

\begin{tabular}{|c|c|c|c|c|c|}
\hline Attitude & Region & $\begin{array}{l}\text { Number of } \\
\text { midwives }\end{array}$ & $\begin{array}{l}\text { *Mean } \\
\text { Rank }\end{array}$ & $\begin{array}{l}\text { Chi } \\
\text { Square }\end{array}$ & Significance \\
\hline \multirow{5}{*}{$\begin{array}{l}\text { The guidelines require } \\
\text { me to refer more often } \\
\text { than is necessary }\end{array}$} & & & & 8.59 & $\mathrm{p}=.035$ \\
\hline & Waikato & 26 & 66.98 & & \\
\hline & Christchurch & 35 & 79.69 & & \\
\hline & Auckland & 65 & 82.30 & & \\
\hline & Wellington & 38 & 100.36 & & \\
\hline \multirow{5}{*}{$\begin{array}{l}\text { The guidelines are a } \\
\text { useful tool when I } \\
\text { consider the need for } \\
\text { referral }\end{array}$} & & & & 16.05 & $\mathrm{p}=.000$ \\
\hline & Wellington & 38 & 64.16 & & \\
\hline & Auckland & 65 & 80.48 & & \\
\hline & Waikato & 26 & 94.89 & & \\
\hline & Christchurch & 35 & 104.81 & & \\
\hline \multirow{5}{*}{$\begin{array}{l}\text { Independent midwives } \\
\text { should care only for } \\
\text { low risk women }\end{array}$} & & & & 10.25 & $\mathrm{p}=.017$ \\
\hline & Wellington & 38 & 74.01 & & \\
\hline & Auckland & 65 & 76.41 & & \\
\hline & Christchurch & 35 & 92.86 & & \\
\hline & Waikato & 26 & 105.59 & & \\
\hline
\end{tabular}

* The scale was graded from agree to disagree. Therefore those with lowest mean rank were most likely to agree with the statement and those regions with the highest mean rank were most likely to disagree.

\section{Relationships with the secondary maternity service}

North Island midwives are less likely than South Island midwives to have a positive primary/secondary interface ( $\mathrm{t} 1.985$, df $304, \mathrm{p}=.048$ ). It was not possible to determine statistically significant differences including all the smaller regions as in several regions the numbers of responding midwives were too small. Five of the 24 regions had fewer than 5 respondents. However the descriptive results dealing with the degree of support in the primary/secondary interface were examined and are detailed in Table 5.11. Midwives, both in the focus groups of this research and in the regions of New Zealand where this research has been presented, have been particularly interested in this information. I have included it in this thesis while recommending caution regarding the minimal numbers in smaller regions. On discussion with midwives in various regions and with maternity managers, there were clearly some 
places where this primary/secondary interface was working much more successfully than others. Hutt Valley and Hastings were known to have problems, and Northland and Southland were thought to be functioning well. This research confirmed this. The mean rank for these regions was calculated using the Kruskal-Wallis Test.

Table 5.11 Degree to which midwives found the primary/secondary interface supportive according to hospital to which they referred

\begin{tabular}{lrr}
\hline Hospital & $\begin{array}{c}\text { Number of } \\
\text { midwives }\end{array}$ & $\begin{array}{l}\text { Mean } \\
\text { rank }\end{array}$ \\
\hline Southland & 5 & 32.8 \\
Whakatane & 4 & 53.4 \\
Northland Base & 15 & 78.3 \\
Nelson & 10 & 105.1 \\
Gisborne & 7 & 117.1 \\
Wellington Women's & 29 & 120.3 \\
North Shore & 14 & 123.1 \\
Dunedin & 15 & 126.8 \\
Tauranga & 9 & 128.3 \\
Wanganui & 11 & 132.1 \\
Rotorua & 11 & 132.1 \\
Middlemore & 19 & 133.9 \\
Wairau & 4 & 140.1 \\
Masterton & 3 & 140.8 \\
Taranaki Base & 9 & 151.6 \\
Greymouth & 5 & 152.2 \\
Christchurch Women's & 35 & 161.0 \\
Timaru & 3 & 189.3 \\
Waikato & 27 & 190.7 \\
Waitakere & 10 & 209.7 \\
Palmerston North & 22 & 213.7 \\
Hutt & 8 & 272.6 \\
Hastings & 8 & 275.3 \\
\hline & 305 & \\
& &
\end{tabular}

Excellent collaboration was correlated with positive attitudes to the referral guidelines $\left(\mathrm{N}=305 \mathrm{r}_{\mathrm{s}}=.169 \mathrm{p}=.003\right)$, and with whether referral was felt to be appropriate $(\mathrm{N}=301$ $\mathrm{r}_{\mathrm{s}}=.263 \mathrm{p}=.000$ ). In particular, collaboration was significantly associated with attitudes to the medico legal environment. Midwives in a collaborative relationship with the secondary service felt less restricted by the medico legal environment $(\mathrm{N}=302$

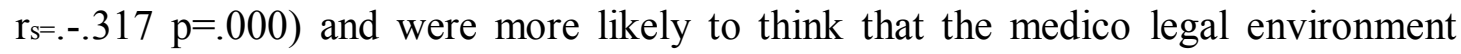
made childbirth safer for women $\left(\mathrm{N}=304 \mathrm{r}_{\mathrm{s}}=.297 \mathrm{p}=.000\right)$. These midwives were also 
more likely to agree that risk management was an important part of their practice $\left(\mathrm{N}=300 \mathrm{r}_{\mathrm{s}}=.175 \mathrm{p}=.002\right)$.

\section{Referral guidelines}

Midwives who thought that the referral guidelines required them to refer more often than necessary also thought that their pattern of referral was less likely to be appropriate to the needs of the women they cared for $\left(\mathrm{N}=303 \mathrm{r}_{\mathrm{s}}=.200 \mathrm{p}=.000\right)$, and that the guidelines were not a useful tool $\left(\mathrm{N}=306 \mathrm{r}_{\mathrm{s}}=-.202 \mathrm{p}=.000\right)$. These midwives were also less likely to work in an area where there was excellent collaboration between primary and secondary service $\left(\mathrm{N}=305 \mathrm{r}_{\mathrm{s}=-.185 \mathrm{p}=.001)}\right.$ and were less supported by obstetricians $\left(\mathrm{N}=307 \mathrm{r}_{\mathrm{s}=-} .150 \mathrm{p}=.008\right)$. Similar correlations were found when midwives were asked whether they agreed that their pattern of referral was appropriate to the needs of the women they cared for. In addition, midwives who thought that they referred appropriately were more likely to state that risk management was an important part of their practice $\left(\mathrm{N}=297 \mathrm{r}_{\mathrm{s}}=.385 \mathrm{p}=.000\right)$.

\section{Risk management}

As well as being more likely to have good collaborative relationships with the secondary maternity service, midwives who felt risk management was an important part of their practice were also more likely to find the referral guidelines useful and to feel that they referred appropriately.

\section{The care of high risk women}

Although there was in general strong disagreement with the statement that independent midwives should care for low risk women only, the midwives who agreed with this statement felt less restricted by the medico legal environment $(\mathrm{N}=305$

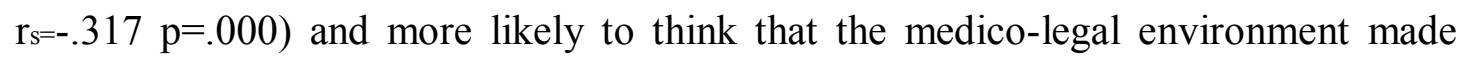
childbirth safer for women $\left(\mathrm{N}=304 \mathrm{r}_{\mathrm{s}=} .297 \mathrm{p}=.000\right)$. It could also be taken from these statistics that midwives who thought that independent midwifery care should be continued into the high-risk area were more medico-legally anxious. They were more likely to think that the medico legal environment restricted practice and less likely to think that it made childbirth safer for women. 


\section{The medico-legal environment}

As mentioned previously a negative response to the medico legal environment was correlated to a negative response to the referral guidelines and also correlated with having a less collaborative secondary care service. There was no correlation between attitudes to the medico legal environment and the importance of risk management as part of midwifery practice.

There were no statistically significant associations between any of the attitudinal statements and age, caseload or experience. There were no differences in the attitudes between direct entry midwives and midwives who had a nursing qualification. Selfemployed midwives were less positive about the primary secondary interface than employed midwives were ( $\mathrm{t} 2.5$, df $301, \mathrm{p}=.013$ ) and were more negative about the risk environment (t 2.98, df 303, $\mathrm{p}=.003$ ).

\section{Summary}

This chapter has provided the detail of the descriptive results of the national survey of New Zealand LMC midwives and has explored some of the interrelationships between the variables in the study. It has elicited a considerable amount of new information about the practices and attitudes of these midwives. Above all it reveals of model of maternity care showing that New Zealand midwives do not provide care for low risk women only but continue care into the secondary service in collaboration with obstetricians. It has established their rate and reasons for referral and has made a start on establishing midwives' attitudes in this setting. It has also looked at possible relationships between the variables in the study, focusing on the referral rate, the caesarean section rate and whether the attitudes of the midwives were associated with their demographic or work pattern characteristics. The next chapter discusses the implications of these findings. 


\section{Chapter 6: Discussion of the Survey}

\section{Introduction}

This study provides important insights into New Zealand midwives' relationship with risk, as expressed in their referral for obstetric consultation practices. The key findings of the study which will be discussed in this chapter are that LMC midwives:

- do not care for 'low risk' women only

- have a referral for obstetric consultation rate of $35 \%$ and a transfer of clinical responsibility rate of $14 \%$

- continue to provide midwifery care into the secondary maternity context

- make most referrals for 'prolonged pregnancy' and 'lack of progress

This chapter begins by providing commentary on the characteristics of the New Zealand LMC workforce. After a discussion of the role of risk screening and referral guidelines, the rates of referral for obstetric consultation and transfer of clinical responsibility are evaluated according to recommended practice. They are then compared with midwifery practice internationally and with referral rates of general practitioners. The referral rates are also evaluated according to the demographic and practice patterns of the midwives, and by looking at the birth outcome statistics of the women in the study. The implications of choice of place of delivery and the degree to which midwives continue care into the secondary care context are discussed.

Two reasons for referral - 'prolonged pregnancy' and 'lack of progress' in labour' are discussed in more detail, as they most aptly represent risk management and are significant contributors to intervention. Preliminary discussion of the findings related to the midwives' attitudes is included in this chapter. A more detailed discussion is presented alongside, and in relation to the focus group data in Chapter 8. There are a large number of other research reports cited in this chapter. Where the term 'this study' is used, it refers to this piece of research. 


\section{The LMC midwifery workforce in New Zealand}

The workforce of LMC midwives in New Zealand is mature and experienced and there continues to be a high proportion of midwives who gained their midwifery qualifications overseas, particularly in the United Kingdom. Although these findings are consistent with previous years, there is still some anxiety expressed regarding issues of education, recruitment and retention (Health Funding Authority, 2000). The distribution of midwives throughout the country is unchanged and the rural/urban split does not look to have altered from the Guilliland (1998) study.

\section{Caseload}

The population characteristics of the midwives, and the degree to which they are free to define the way they practice are reflected both in the extent to which they are selfemployed and in their caseloads. Midwives of this age are highly likely to be parents of dependant children. Self-employment facilitates flexibility of caseload and work organisation, so has the potential to be particularly appropriate for women with dependants. Although caseload in the study is a normally distributed variable, $43 \%$ of the midwives had caseloads below the NZCOM's recommendations for full-time practice and $27 \%$ had the recommended full-time caseload. A surprising $30 \%$ however had a caseload over the NZCOM recommendation of 4 to 5 women per month (New Zealand College of Midwives Inc, 1993). This is an important finding, as there have been no previous reports of LMC midwifery caseload. The findings from the survey, that midwives with higher caseloads have lower referral rates, also point to this issue as being of interest. Possible explanations for high caseloads are a shortage of midwives and/or the need or wish for a higher income. Midwives in the Auckland region for example, where there is pressure both of a higher cost of living and of workforce shortage, have an increased incidence of high caseloads. Nationally, the rise in the cost of living has not been followed by a corresponding rise in LMC funding. Since 1993, when LMC funding for independent midwives was first introduced, there has been only one small increase in funding. It may be timely for the NZCOM to review their recommendations for caseload in order either to amend it, or to request an increase in LMC funding, and/or action on recruitment and retention. As expected, caseload was significantly associated with years of experience. More 
experienced midwives have higher caseloads. However experience was not associated with region, or with any other variables in the study.

\section{Workforce changes}

Although the age, experience and original place of registration of midwives remain relatively stable, the main changes to the midwifery workforce over the last five years relate to the growing proportion of midwives who do not have a nursing qualification, and the shift in practice from shared care with doctors to midwifery-led care. This shift is expected, as all midwifery education in New Zealand is now based on the direct entry model. The growth in LMC midwifery has happened because of a number of contributing factors. It may be the mark of a maturing and more confident workforce moving into autonomous practice. It also marks a change in the attitudes of childbearing women in New Zealand, now prepared to have maternity care without medical input and keen to have continuity (The National Health Committee, 1999). Of significant relevance is the marked decrease in the numbers of general practitioners (GPs) providing an LMC service.

\section{Risk screening}

One of the key findings of this study is that New Zealand LMC midwives do not care for 'low risk' women only. In New Zealand, women have considerable freedom to choose who will provide their maternity care. They can choose a midwife, a GP or an obstetrician. At booking, there is no formal risk screening process where place of birth or care provider is allocated. The extent to which early antenatal screening does not formally occur is reflected in this study by the degree to which transfer of clinical responsibility occurs at or before 20 weeks gestation. This occurred for only 18 women and represents $0.4 \%$ of all the midwives' bookings. However there does appear to be some informal screening process. The Report on Maternity (New Zealand Health Information Service, 2003) states that private obstetricians are more likely to deal with medium-risk women and hospital obstetricians with higher-risk women. This statement is supported somewhat by the extent to which women under the care of obstetricians have an increased rate of caesarean section (Table 6.1). 
Table 6.1 Type of birth by original LMC in 2000

\begin{tabular}{|c|c|c|c|c|c|c|c|c|}
\hline \multirow[t]{2}{*}{ Birth type } & \multicolumn{2}{|c|}{ Midwife } & \multicolumn{2}{|c|}{ GP } & \multicolumn{2}{|c|}{ Obstetrician } & \multicolumn{2}{|c|}{ Total } \\
\hline & No & $\%$ & No & $\%$ & No & $\%$ & No & $\%$ \\
\hline Caesarean & & & & & & & & \\
\hline Section & 3767 & 16.6 & 1061 & 19.1 & 1384 & 29.7 & 6212 & 8.9 \\
\hline $\begin{array}{l}\text { Vaginal } \\
\text { breech }\end{array}$ & 192 & 0.8 & 37 & 0.7 & 57 & 1.2 & 286 & 5.9 \\
\hline $\begin{array}{c}\text { Assisted } \\
\text { vaginal }\end{array}$ & 2440 & 10.7 & 596 & 10.7 & 548 & 11.6 & 3584 & 10.9 \\
\hline $\begin{array}{l}\text { Normal } \\
\text { birth }\end{array}$ & 16285 & 71.8 & 3845 & 69.4 & 2723 & 57.8 & 22853 & 69.4 \\
\hline Total & 22684 & 100.0 & 5539 & 100.0 & 4712 & 100.0 & 32935 & 100.0 \\
\hline
\end{tabular}

$* 30 \%$ of data missing (New Zealand Health Information Service, 2003)

As the reasons for referral in this study show (e.g. pre-existing medical condition and previous caesarean section), there were considerable numbers of the midwives' clients who could not be classified as low risk. The degree to which midwives care for the full range of the maternity population is also evident when one looks at the demographic characteristics of the referred women. They are matched by age and parity with the general childbearing population. Pacific and Asian women are less likely to have midwifery-led care.

New Zealand's practice of not having a risk screening process at booking is supported by comments in the literature. As pointed out in Chapter 2 the identification of risk factors and screening for these factors suffers from low sensitivity and low specificity (Saxell, 2000; Schettler et al., 1999; Yuster, 1995). It does however continue to be practised, as many of the studies cited later in this chapter illustrate. In 2002, a risk scoring system developed in New Zealand was still being assessed for applicability for the United Kingdom, although its use in New Zealand was very limited (Knox, Sadler, \& Patterson, 1993; Mohamed, Martin, \& Haloob, 2002). It too failed to predict poor pregnancy outcome.

Alexander and Keirse (1989) propose that risk screening for the individual woman is nothing more than tautology.

When you include a diagnosis such as previous caesarian section for example and give it a risk score you may give it unwarranted emphasis. Risk screening performs poorly because risk factors are not causative of the predicted outcome. The other confounding problem with risk 
scoring is that they mix up potential and existing problems. The best and most precise risk prediction is made at a time when there is no further need for it, whereas the much more necessary early identification is notoriously imprecise ( $\mathrm{p} 352$ ).

It is also thought that risk screening has a negative impact on women. For the individual woman labelled as being at increased risk, both the threat of poor outcome and the inability to change its course may cause feelings of guilt and inadequacy which is unlikely to improve maternity outcome (Alexander \& Keirse, 1989; Stahl \& Hundley, 2003). Risk screening does aim to identify the few individuals who are likely to experience an adverse outcome. What it does not do however is identify those whose chances of a good outcome are so good that they are unlikely to be improved by whatever obstetrics offers. It also fails to identify any risk factors other than physical. Social and cultural factors are usually ignored. Those scores which do try to look at other issues such as socioeconomic factors, face significant ethical dilemmas.

Wagner (1994) poses four further limitations on the notion of risk screening.

- They are based on the medical model that birth is risky and dangerous. The pregnant woman incorporates this into her perception of her pregnancy

- It focuses on the birth at the expense of all other areas of care and weakens the interest of other professionals involved

- The pregnant woman becomes a passive patient and the obstetrician a baby advocate

- The high tech hospital becomes the pinnacle of care, perpetuating the spiral of more risk and more intervention

As stated previously, the World Health Organisation has also changed its position on risk screening in favour of improving the quality and accessibility of maternity care. Oakley and Houd (1990) propose an alternative framework to the risk approach. They support the call to rename risk as 'complex need' and isolate the important ingredients in its management as: effective primary care which is adapted to individual needs, cross disciplinary cooperation, and care provided in a 
comprehensive way which is evaluated both quantitatively and qualitatively. They propose that the results based on this primary prevention approach need not be worse than by the risk approach. Saxell (2000), also critiques the risk screening approach, and proposes a risk prevention and assessment model for midwifery practice where risk is dealt with in a holistic, continuous way. She cites the work of two leading New Zealand midwives, Guilliland, (1990) and Donley(1993), as presenting an alternative model; the New Zealand model.

Studies which have evaluated the outcomes of midwifery-led care where midwives provide care for a mixed risk population illustrate that the outcomes are no worse and in fact may be considerably better than obstetric led care (Biro, Waldenstrom, \& Pannifex, 2000; Declercq, 1995; Homer et al., 2001; Rowley, Hensley, Brinsmead, \& Wlodarczyk, 1995). The outcomes of this study, and the Report on Maternity would confirm this. The caesarean section rate in this study was $11.8 \%$ and that of the midwifery LMC population in the year of the study was $16.6 \%$. The caesarean section rate of the total childbearing population was 20.8\% (New Zealand Health Information Service, 2003).

\section{Referral and transfer of clinical responsibility}

The New Zealand context in which referral and transfer of clinical responsibility takes place is marked by an environment in which the woman's choice is paramount and in which the relationship that the midwife has with the woman is central. There is no formal screening process at booking where caregiver or place of birth is allocated. The midwife has access to secondary facilities and usually has secondary maternity skills. She does not look after only 'low risk' women. These are key findings of the study and are important to keep in mind when considering the appropriateness of referral practices.

\section{The referral rate}

This study has found a midwifery referral rate of $35 \%$. One essential question of this study is whether this rate of referral might be considered too high or too low, or whether in fact it might be considered as a benchmark for referral. In order to attempt to answer this question it is useful both to look at what recommendations for referral might be, and to compare this rate with that of international studies of midwifery-led 
care and with the rate of referral of general practitioners. The rate can also be explored by assessing its relationships with the key demographic and practice patterns of the midwives to ascertain what might impact on it.

\section{Recommendations and guidelines for referral}

The New Zealand Maternity Service reference document states:

In New Zealand, it is considered that approximately 25-30 percent of women will need to transfer to the care of an obstetrician, with some of these transfers occurring for less than $24 \mathrm{hrs}$. A further 20 percent of women are estimated to have a clinical need for consultation with a specialist obstetrician. These estimations are based on an analysis of case mix prior to the move to a population-based funding model for secondary maternity services. (Health Funding Authority, 2000, p60).

This statement would indicate that $45-50 \%$ of women need at least one referral for obstetric consultation. Given the fact that the midwives in this study had a $35 \%$ referral rate and that midwives in that year were LMCs for $70 \%$ of New Zealand women (New Zealand Health Information Service, 2003), one might conclude that this referral rate is too low. However it must be remembered that $30 \%$ of women are cared for by doctors, mainly obstetricians, and although we know from this study what the case-mix of midwives is, there is no information on the case-mix of either general practitioners or obstetricians. The above statement from the Health Funding Authority might not reflect current practice modes and should be reconsidered, as no information was provided in the document about how case-mix was analysed. Given the poor state of New Zealand perinatal data at the time, this analysis was likely to be particularly haphazard. The analysis probably spread risk status evenly across all caregivers.

By 2003, official policy more closely reflected what was happening in practice as can be seen in the comment that private obstetricians are more likely to deal with medium-risk women and hospital obstetricians with higher-risk women (New Zealand Health Information Service, 2003). It is expected therefore, that the case mix of women who book with an LMC midwife would include a lower proportion of 'at risk' women, and that a referral rate of $35 \%$ might be entirely appropriate. However, in 
order to be definitive about this, more detail is required about the case-mix of both midwives and doctors. An effective national database would help to solve this problem. At this stage therefore there is no external standard against which to accurately measure this rate of referral. Internationally the World Health Organisation estimated that at least $15 \%$ of pregnant women will require specialist medical care in order to avoid death or disability (World Health Organisation, 1994). Therefore, given the findings of this study, that $14.3 \%$ of women have responsibility for their care transferred to obstetricians, the referral and transfer rates are within acceptable limits.

\section{Comparisons of referral rates with other midwives and with GPs}

There was only one study found in the literature that specifically addressed referral rates of midwives, and only two, both from North America, which addressed GPs' obstetric referral practices. In order to discover other practitioners' referral rates, studies that provided outcomes of midwifery-led or GP-led maternity care were examined. Those studies found which provided referral rates are summarised in the following table (Table 6.2). The studies in this table vary considerably in case mix, practice patterns and the risk status of the women. Although the risk status of the women is provided, different risk categorisations will have been used. In general, these were not detailed in the reports. I have also included studies which provided GP or family physician referral rates, as in New Zealand the replacement of GP care by midwifery care has meant that midwives care for a population very similar to that of the GPs. Comparison is appropriate. The New Zealand referral guidelines apply equally to GPs and midwives. 
Table 6.2 Summary of referral rates found in national and international studies

\begin{tabular}{|c|c|c|c|c|c|}
\hline & Type of study & Practitioners & Risk status* & $\begin{array}{l}\text { Routine } \\
\text { obstetric } \\
\text { visit } \\
\end{array}$ & Referral/transfer** \\
\hline \multicolumn{6}{|l|}{ United Kingdom } \\
\hline $\begin{array}{l}\text { (Hundley et al., } \\
\text { 1994) } \\
\text { (Scotland) }\end{array}$ & $\begin{array}{l}\text { RCT of midwife-led care vs } \\
\text { consultant care }\end{array}$ & Midwives & $\begin{array}{l}\text { Low risk with strict protocols } \\
\text { for booking, admission and } \\
\text { transfer }\end{array}$ & $\begin{array}{l}\text { Not } \\
\text { known }\end{array}$ & $\begin{array}{l}45 \% \text { transferred to specialist } \\
\text { care; } 3 \% \text { in antenatal period, } \\
22 \% \text { in intrapartum }\end{array}$ \\
\hline $\begin{array}{l}\text { (MacVicar et al., } \\
\text { 1993) } \\
\text { (England) }\end{array}$ & $\begin{array}{l}\text { RCT of Home-from Home } \\
\text { delivery scheme vs. consultant } \\
\text {-led care }\end{array}$ & Midwives & $\begin{array}{l}\text { Low risk. Only } 44 \% \\
\text { considered low risk enough } \\
\text { to enter the study }\end{array}$ & Yes & $\begin{array}{l}45 \% \text { of these screened women } \\
\text { were transferred to consultant } \\
\text { care at some stage of the } \\
\text { pregnancy or labour. } 23 \% \\
\text { antenatally, } 22 \% \text { intrapartum }\end{array}$ \\
\hline $\begin{array}{l}\text { (Flint \& } \\
\text { Poulengeris, 1987) } \\
\text { (England) }\end{array}$ & $\begin{array}{l}\text { RCT of team midwifery vs. } \\
\text { standard care }\end{array}$ & Midwives & Low risk & Yes & $\begin{array}{l}\text { All women had a routine } \\
\text { obstetric consultation at } 36 \\
\text { weeks. Transfers not provided }\end{array}$ \\
\hline $\begin{array}{l}\text { (Kean, Liu, \& } \\
\text { Macquisten, 1996) } \\
\text { (England) }\end{array}$ & Historical cohort study & $\begin{array}{l}\text { Documentation } \\
\text { of low risk } \\
\text { women }\end{array}$ & $\begin{array}{l}\text { Low risk Under } 50 \% \text { of } \\
\text { women considered low risk } \\
\text { enough for community care. }\end{array}$ & & $\begin{array}{l}60 \% \text { of the women needed } \\
\text { obstetric contact during the } \\
\text { pregnancy or labour }\end{array}$ \\
\hline $\begin{array}{l}\text { (Chapman, Jones, } \\
\text { Spring, DeSwiet, } \\
\text { \& Chamberlain, } \\
\text { 1986) (England) }\end{array}$ & $\begin{array}{l}\mathrm{RCT} \text { of delivery in a birth } \\
\text { room within the labour ward }\end{array}$ & Not available & Low risk multips & & $\begin{array}{l}23.6 \% \text { were withdrawn from } \\
\text { the study mainly because they } \\
\text { developed complications }\end{array}$ \\
\hline
\end{tabular}




\begin{tabular}{|c|c|c|c|c|c|}
\hline $\begin{array}{l}\text { (Klein, Llloyd, } \\
\text { Redman, Bull, \& } \\
\text { Turnbull, 1983) } \\
\text { (England) }\end{array}$ & Group comparison & $\begin{array}{l}\text { GPs and } \\
\text { midwives with } \\
\text { consultant -led } \\
\text { care }\end{array}$ & Low risk & No & $\begin{array}{l}48 \% \text { of primips and } 25 \% \text { of } \\
\text { primips were transferred }\end{array}$ \\
\hline $\begin{array}{l}\text { (Tucker et al., } \\
\text { 1996) } \\
\text { (Scotland) }\end{array}$ & $\begin{array}{l}\text { RCT of routine antenatal care } \\
\text { vs. obstetrician led shared care. }\end{array}$ & $\begin{array}{l}\text { Midwives and } \\
\text { GPs }\end{array}$ & Low risk & No & $\begin{array}{l}32.4 \% \text { had their care } \\
\text { transferred to obstetricians }\end{array}$ \\
\hline $\begin{array}{l}\text { (Turnbull, Holmes, } \\
\text { \& Shields, 1996) } \\
\text { (Scotland) }\end{array}$ & $\begin{array}{l}\text { RCT of midwife managed care } \\
\text { vs. shared care }\end{array}$ & Midwives & Low risk & No & $\begin{array}{l}65.6 \% \text { of women who had } \\
\text { midwifery-led care had their } \\
\text { care transferred, } 32.8 \% \text { of } \\
\text { these temporarily, } 32.8 \% \\
\text { permanently transferred }\end{array}$ \\
\hline $\begin{array}{l}\text { (Street, Gannon, \& } \\
\text { Holt, 1991) } \\
\text { (England) }\end{array}$ & $\begin{array}{l}\text { Before and after comparison of } \\
\text { all antenatal care for low risk } \\
\text { women in the community }\end{array}$ & $\begin{array}{l}\text { Midwives and } \\
\text { GPs }\end{array}$ & Low risk & No & $\begin{array}{l}49.2 \% \text { transfer of care to } \\
\text { obstetricians }\end{array}$ \\
\hline $\begin{array}{l}\text { (Law \& Lam, } \\
\text { 1999) } \\
\text { (England) }\end{array}$ & $\begin{array}{l}\text { RCT comparing midwife } \\
\text { managed care with obstetric } \\
\text { care }\end{array}$ & Midwives & Low risk & & $\begin{array}{l}26.6 \% \text { transfer of care to } \\
\text { obstetricians }\end{array}$ \\
\hline $\begin{array}{l}\text { (Benjamin, Walsh, } \\
\& \text { Taub, 2001) } \\
\text { (England) }\end{array}$ & $\begin{array}{l}\text { A prospective, non-randomised } \\
\text { clinical trial of partnership } \\
\text { caseload midwifery and } \\
\text { conventional midwifery }\end{array}$ & Midwives & & & \\
\hline
\end{tabular}




\begin{tabular}{|c|c|c|c|c|c|}
\hline $\begin{array}{l}\text { (Page, McCourt, } \\
\text { Beake, Vail, \& } \\
\text { Hewison, 1999) } \\
\text { (England) }\end{array}$ & $\begin{array}{l}\text { Prospective comparative study } \\
\text { of one-to-one midwifery with } \\
\text { standard care }\end{array}$ & Midwives & Low risk & & \\
\hline $\begin{array}{l}\text { (Boulton, Chapple, } \\
\& \text { Saunders, 2003) }\end{array}$ & $\begin{array}{l}\text { Evaluation report of a birth } \\
\text { centre }\end{array}$ & Midwives & Low risk & No & $\begin{array}{l}24 \% \text { antenatal transfer } \\
12 \% \text { intrapartum transfer }\end{array}$ \\
\hline $\begin{array}{l}\text { (Sandall, Davies, } \\
\text { \& Warwick, 2001) } \\
\text { (England) }\end{array}$ & $\begin{array}{l}\text { Evaluation report of a group } \\
\text { midwifery practice }\end{array}$ & Midwives & Mixed risk. & No & Not provided \\
\hline $\begin{array}{l}\text { Europe } \\
\text { Waldenstrom, } \\
1997 \# 460 ; \\
\text { (Sweden) }\end{array}$ & $\begin{array}{l}\text { RCT of in-hospital birth centre } \\
\text { vs. with standard care }\end{array}$ & $\begin{array}{l}\text { Team of } \\
\text { midwives }\end{array}$ & $\begin{array}{l}\text { Low risk but includes } \\
\text { previous } \mathrm{C} / \mathrm{S} \text { and perinatal } \\
\text { death }\end{array}$ & No & $\begin{array}{l}\text { Referral rates were } 13 \% \\
\text { antenatally, } 19 \% \text { intrapartum } \\
\text { and } 2 \% \text { postpartum.. }\end{array}$ \\
\hline $\begin{array}{l}\text { (Wiegers, Keirse, } \\
\text { van der Zee, \& } \\
\text { Berghs, 1996) } \\
\text { (The Netherlands) }\end{array}$ & $\begin{array}{l}\text { Prospective data analysis, } \\
\text { home and hospital birth }\end{array}$ & Midwives & Low risk & No & $\begin{array}{l}13.8 \% \text { antenatal transfer } \\
16.5 \% \text { transfer to hospital rate }\end{array}$ \\
\hline $\begin{array}{l}\text { (Smit, Scherjon, \& } \\
\text { Treffers, 1997) } \\
\text { (The Netherlands) }\end{array}$ & Prospective cohort study & Midwives & Low risk & No & \\
\hline
\end{tabular}




\begin{tabular}{|c|c|c|c|c|c|}
\hline $\begin{array}{l}\text { North America } \\
\text { (Janseen et al., } \\
2002) \\
\text { (Canada) }\end{array}$ & $\begin{array}{l}\text { Matched comparison of } \\
\text { planned home births, hospital } \\
\text { midwife-attended births }\end{array}$ & Midwives & Low risk & No & $\begin{array}{l}36.7 \% \text { of home births and } \\
40.7 \% \text { of hospital births were } \\
\text { referred for specialist care in } \\
\text { labour. }\end{array}$ \\
\hline $\begin{array}{l}\text { (Fraser et al., } \\
2000) \\
\text { (Canada) }\end{array}$ & $\begin{array}{l}\text { Matched comparison of } \\
\text { midwifery-led care in a birth } \\
\text { centre with medical care }\end{array}$ & Midwives & Low risk & No & $15 \%$ transfer to secondary care \\
\hline $\begin{array}{l}\text { (Tyson, 1991) } \\
\text { (Canada) }\end{array}$ & $\begin{array}{l}\text { Descriptive study of home } \\
\text { births }\end{array}$ & Midwives & Low risk & No & $\begin{array}{l}16.5 \% \text { transfer during labour } \\
\text { and postpartum }\end{array}$ \\
\hline $\begin{array}{l}\text { (Harvey, Jarrell, } \\
\text { Brant, Stainton, \& } \\
\text { Rach, 1996) } \\
\text { (Canada) }\end{array}$ & $\begin{array}{l}\text { RCT of midwifery -led care in } \\
\text { a birth room }\end{array}$ & Midwives & Low risk & & $\begin{array}{l}1 \% \text { antenatally, } 26 \% \\
\text { intrapartum }\end{array}$ \\
\hline $\begin{array}{l}\text { (Anderson \& } \\
\text { Murphy, 1995) } \\
\text { (USA) }\end{array}$ & $\begin{array}{l}\text { A retrospective descriptive } \\
\text { study of planned home births }\end{array}$ & $\begin{array}{l}\text { Certified nurse- } \\
\text { midwives }\end{array}$ & Low risk & Not stated & $\begin{array}{l}6 \% \text { transferred antenatally } \\
8.2 \% \text { in labour }\end{array}$ \\
\hline $\begin{array}{l}\text { Australasia } \\
\text { (Biro \& Lumley, } \\
\text { 1991) } \\
\text { (Australia) }\end{array}$ & Cohort study & Midwives & Low risk & Yes & $\begin{array}{l}\text { Two routine visits with } \\
\text { obstetricians. Transfer rate not } \\
\text { supplied }\end{array}$ \\
\hline
\end{tabular}




\begin{tabular}{|c|c|c|c|c|c|}
\hline $\begin{array}{l}\text { (Giles, Collins, } \\
\text { Ong, \& } \\
\text { MacDonald, 1992) } \\
\text { (Australia) }\end{array}$ & $\begin{array}{l}\text { RCT comparing antenatal care } \\
\text { by midwives with obstetricians }\end{array}$ & Midwives & Low risk & Yes & $100 \%$ consultation rate \\
\hline $\begin{array}{l}\text { (Rowley et al., } \\
\text { 1995) } \\
\text { (Australia) }\end{array}$ & $\begin{array}{l}\text { RCT of team midwifery vs. } \\
\text { standard care }\end{array}$ & $\begin{array}{l}\text { Midwives with } \\
\text { consultant input }\end{array}$ & Mixed risk & Yes & $\begin{array}{l}\text { All women had } 3 \text { antenatal } \\
\text { consultations }\end{array}$ \\
\hline $\begin{array}{l}\text { (Biro et al., 2000) } \\
\text { (Australia) }\end{array}$ & $\begin{array}{l}\text { RCT of continuity of } \\
\text { midwifery care vs standard } \\
\text { care }\end{array}$ & $\begin{array}{l}\text { Midwives with } \\
\text { obstetric } \\
\text { collaboration }\end{array}$ & Mixed risk & Yes & $\begin{array}{l}\text { All women had at least one } \\
\text { consultation with an } \\
\text { obstetrician }\end{array}$ \\
\hline $\begin{array}{l}\text { (Homer, Matha, } \\
\text { Jordan, Wills, \& } \\
\text { Davis, 2001) } \\
\text { (Australia) }\end{array}$ & $\begin{array}{l}\text { RCT comparing community } \\
\text { based care of midwives and } \\
\text { obstetricians with standard } \\
\text { care }\end{array}$ & $\begin{array}{l}\text { Midwives and } \\
\text { obstetricians }\end{array}$ & Mixed risk & Yes & $100 \%$ consultation rate \\
\hline (Griew, 2003) & $\begin{array}{l}\text { A study of Australian birth } \\
\text { centres }\end{array}$ & Midwives & Low to moderate risk & $\begin{array}{l}\text { Both self } \\
\text { referral } \\
\text { and some } \\
\text { medical } \\
\text { screening }\end{array}$ & $\begin{array}{l}\text { Transfer rates vary from } 30 \% \\
\text { to } 50 \%\end{array}$ \\
\hline $\begin{array}{l}\text { (Virtue, 2000) } \\
\text { (New Zealand) }\end{array}$ & Annual Practice report & Midwives & Mixed risk & No & $22 \%$ transfer of care rate \\
\hline
\end{tabular}




\begin{tabular}{|c|c|c|c|c|c|}
\hline $\begin{array}{l}\text { General } \\
\text { Practitioners } \\
\text { (Tilyard, Williams, } \\
\text { Seddon, Oakley, \& } \\
\text { Murdoch, 1988) } \\
\text { (New Zealand) }\end{array}$ & Descriptive study & GPs & Mixed risk & & \\
\hline $\begin{array}{l}\text { (Bryce et al., 1990) } \\
\text { (England) }\end{array}$ & Case review & GPs & Not stated & No & $49 \%$ transfer to obstetricians \\
\hline $\begin{array}{l}\text { (Radomsky, 1995) } \\
\text { (Canada) }\end{array}$ & Retrospective chart review & $\begin{array}{l}\text { Family } \\
\text { physicians }\end{array}$ & Mixed risk & No & $\begin{array}{l}12 \% \text { antenatal transfer rate } \\
5 \% \text { intrapartum consultation } \\
\text { rate for primips and } 19 \% \text { for } \\
\text { multips }\end{array}$ \\
\hline $\begin{array}{l}\text { (Reid, Carroll, } \\
\text { Ruderman, \& } \\
\text { Murray, 1995) } \\
\text { (Canada) }\end{array}$ & Retrospective chart review & $\begin{array}{l}\text { Family } \\
\text { physicians }\end{array}$ & Mixed risk. & No & $\begin{array}{l}34.8 \% \text { antenatal referrals } \\
32.6 \% \text { intrapartum referrals }\end{array}$ \\
\hline $\begin{array}{l}\text { (Rosenthal, } \\
\text { Holden, \& } \\
\text { Woodward, 1990) } \\
\text { (USA) }\end{array}$ & Retrospective chart review & $\begin{array}{l}\text { Rural family } \\
\text { physicians }\end{array}$ & Mixed risk & No & $\begin{array}{l}36 \% \text { referral for consultation } \\
18 \% \text { transfer of care }\end{array}$ \\
\hline $\begin{array}{l}\text { (Sinusas, } 2000 \\
\# 519) \\
\text { (USA) }\end{array}$ & Survey & $\begin{array}{l}\text { Family } \\
\text { physicians }\end{array}$ & Mixed risk & No & $\begin{array}{l}\text { Referral for consultation rates } \\
\text { varied form } 12.8 \text { to } 49.6 \%\end{array}$ \\
\hline
\end{tabular}




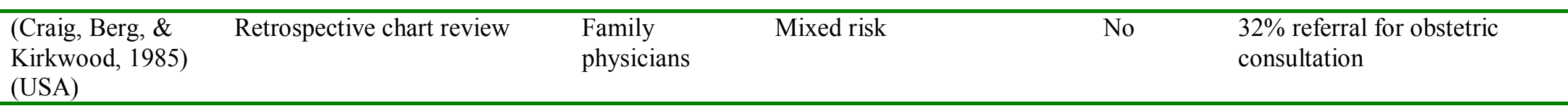

(USA)

* The studies were seldom explicit about what constituted high and low risk status. Risk criteria were often developed by the research team. Studies that included mixed risk women were not explicit about the extent of the case-mix. There may be considerable variation in risk status.

** The use of the terms 'referral' and 'transfer' were often used interchangeably. Many of the studies were of birth or primary birthing centres where a referral entailed transfer. 
The two areas of particular interest in these studies are the extent to which the midwives cared for low risk women only, and their rates of referral or transfer of care. There is a marked difference between the midwives in the UK based studies cited above and New Zealand midwives regarding the risk status of the women for whom they care. The UK midwives in the studies tended to focus on the place of birth i.e. primary, community based maternity facilities, thus restricting the type of women for whom they provided care. This differs markedly from the New Zealand model in which the choice of place of birth is secondary to the choice of caregiver. Although the referral rates varied, they were in general very high. Hall (2003) in her more recent study of 55 free standing maternity units in England also found that there were large differences in the transfer rates. She found, however that the average intrapartum transfer rate was $15.8 \%$, and that the total transfer rate (intrapartum and postpartum) was $18.3 \%$. She found that there was considerable diversity in admission criteria and transfer rates, and that risk screening was variously defined and executed.

Two of the UK based studies (Hundley et al., 1994; MacVicar et al., 1993) were cited by The World Health Organisation in their 1996 document 'Care in Normal Birth' (Maternal and Newborn Health/Safe Motherhood Unit, 1996). These studies were used to support the contention that referral rates of $20 \%$ during the antenatal stage, and a further $20 \%$ during the intrapartum stage are expected. This would suggest that the referral rates of New Zealand LMC midwives are low. Both these studies had overall transfer rates of $45 \%$, yet in my study, of mixed risk women, the referral rate is only $35 \%$ and transfer of responsibility rate is $14 \%$. For the WHO to suggest that the referral rates in the cited studies are usual would indicate that there are very few women (only 40\%) who do not require obstetric led care. I consider these high rates challenge the viability of midwifery-led care within primary birth units. The latest WHO recommendations for 'Care in Normal Birth'(World Health Organisation, 2004), have omitted any comment about referral or transfer rates, which in my opinion is more appropriate given the lack of focused research on this issue. It seems from this that the jury remains out on how much referral is acceptable. Further evidence of high transfer rates was found in Waldestrom and Turnbull's (1998) systematic review of seven RCTs which compared continuity of midwifery care with standard maternity care. Transfer rates were provided in seven of the studies and ranged from $55 \%$ to $73 \%$. 
The extent to which the experimental models detailed in Table 6.2 reflected current midwifery practice was unclear. However the control groups in both the RCTs and matched cohort studies were those who received 'normal care' and this illustrates that the usual model of care was not midwifery-led. Another explanation of the highly risk-screened nature of the study populations might be that there was difficulty in obtaining ethical approval for studies of midwifery-led care in an unscreened population. The tendency for higher rates of transfer in the experimental studies than in the descriptive studies may also be an indication of research effect. Hall's (2003) study was very useful in providing a description of midwifery practice not constructed for research purposes. The lower average rate of transfer supports the contention that the transfer rates in the experimental studies might be a factor of the research design rather than a real-world issue.

It was useful to be able to visit the UK in person on a study tour, and I met with midwives from the Albany Midwifery Practice in South London. This practice closely resembled the way New Zealand LMC midwifery was provided. The risk status of the women they cared for and the way they referred and continued care into the secondary environment, was very similar to New Zealand. Unfortunately this information was not collected in the evaluation of their practice (Sandall, Davies \& Warwick, 2001). One thing which did differ from the New Zealand model was the extent to which they felt isolated and pressured, as no other midwives in their area practiced in a similar way. It would seem that this model of midwifery-led care remains a minority model within the UK and that there is general consensus that, where it exists, midwifery-led care is available mainly for screened low-risk women with an increased focus on the choice of place of birth rather than on the choice of caregiver. I am also aware that there is a model of independent midwifery in the UK that more closely matches the New Zealand model. However this is not state funded and therefore remains a minority model.

Although women in the Swedish and Dutch studies were also categorised as low risk, their referral rates were noticeably lower. Although the model of midwifery-led care is dominant in the Netherlands, Denmark and Sweden (Mckay, 2000), there were no national referral or transfer rates available. Apart from the Jansen, Lee, Ryan el al (2002) study, all the midwifery practices in the North American studies revealed 
lower transfer rates. These were studies also conducted in home or birth centres, so restricted the choices for women as they were a screened population. One might challenge a model of midwifery care that excludes such a large proportion of the population.

It would seem that we need to move down to the antipodes to see a shift in midwives' attitudes towards women's risk status. All but one of the Australian studies cited included mixed risk women (Biro et al., 2000; Bradshaw, Lewis, \& Steer, 1995; Homer et al., 2001; Kenny, Brodie, Eckerman, \& Hall, 1994; Rowley et al., 1995). In all these studies, the women had a routine consultation with an obstetrician during the pregnancy, in effect a $100 \%$ referral for obstetric consultation rate. Although it was clear that the women designated as high risk in these studies benefited from continuity of midwifery care, there is little evidence that low risk women benefit from obstetric consultation (Tucker et al., 1996). It may have been that a routine referral for all women was a prerequisite in order to obtain support from the medical profession for the research. However, it is possible that communication engendered by these referrals was important in generating positive collaborative relationships. The decision to include women with higher risk status in a model of midwifery-led care differs markedly from the UK community based model and more closely matches the New Zealand approach. Griew's (2003) study of birth centre care in Australia noted that women with moderate risk could be admitted to some birth centres. This study also recorded variable transfer rates.

The studies of GP-led care are more helpful in comparing referral rates in that they care for a mixed risk population and are based in the community. Their rates of referral are most similar to those in the New Zealand midwifery model.

Risk screening prevents a large number of women from accessing community-based continuity of care. Risk assessment right through the pregnancy and delivery with appropriate referral, leads to good outcomes and higher levels of satisfaction (World Health Organisation, 2004). The New Zealand model most closely fits this approach. The review of the studies in Table 6.2 has confirmed that midwifery led care in New Zealand has a combination of distinctive characteristics which together make it unique: higher risk women are not excluded from care, there is no routine consultation 
or screening process at booking, and midwives continue care when secondary care is required. There is clearly a focus on choice of caregiver rather than on place of birth. The impact and distinctiveness of New Zealand's model of maternity care is further highlighted by the fact that over $70 \%$ of New Zealand women have this type of care. This is not simply a model for a small study population.

The examination of these studies however has not assisted greatly in the assessment of whether New Zealand midwives' referral rates are appropriate. The New Zealand rate is much lower than most of those examined in the United Kingdom studies and more in line with those in Europe and North America. This is interesting, given the large numbers of midwives in this study who were educated in the UK and the historical links we have had with UK obstetric practice.

\section{Commentary in the literature on referral rates}

The commentary on referral rates included in many of the studies cited above varied considerably. Referral rates and outcomes were used both to justify the validity of maternity care being provided by midwives and GPs (MacVicar et al., 1993; Radomsky, 1995; Reid et al., 1995; Smith, 1998), and to challenge whether care outside secondary obstetric care was safe especially for primigravid women (Hundley et al., 1994; Street et al., 1991). Some challenged mandatory consultations for women at low risk (Reid et al., 1995; Tucker et al., 1996) and others commented on the appropriateness of existing referral criteria. There was also comment made on the wide variation of referral rates and the difficulty of monitoring or assessing these (Hall, 2003). Despite this, there was reasonable consensus that referral criteria were important and that there needed to be a well supported framework for consultation.

The only study found which assessed appropriateness of referral practices was that by Tucker et al (1996). This was a randomised controlled trial assessing whether obstetricians should see women with normal pregnancies and concluded that this was not necessary. There was no evidence in any of the other studies which either supported or challenged the referral rates themselves. In general they were accepted as a fait accompli. One study stated that, given the wide variation in referral rates and the difficulty in assessing their appropriateness, studies examining them should be 
replaced by studies which examined referral practices rather than rates (Reid et al., 1995).

This comment is also supported by O'Donnell(2000) in her comprehensive review of GP referral practices. She stated that the rates themselves do not say anything about the appropriateness of the referrals, nor even whether the norm is appropriate. She found that there was a lack of consensus about what constituted appropriate referral and the use of guidelines had only limited success in altering referral behaviour. She stated, "Pressure on GPs to review their referral behaviour and the use of guidelines may reduce their willingness to tolerate uncertainty and manage problems in primary care, resulting in an increase in referrals to secondary care" ( $p$ 470).

She concluded that the use of referral rates to measure performance would be misguided, and that activity should concentrate on increasing the number of appropriate referrals, regardless of rate.

Referral rates may be better used as a catalyst to stimulate dialogue and joint working between primary and secondary care colleagues. Such an approach may not reduce the number of referrals per se, but could result in more appropriate use of secondary care facilities ( $p$ 470).

\section{Referral patterns and midwifery characteristics}

Referral patterns can also be evaluated by assessing the internal relationships between the characteristics and practice patterns of the midwives and the referral rates. In this study, caseload was the only variable that had any association with referral rate: the higher the caseload the lower the referral rate. This was particularly true for those who had workloads over the NZCOM recommendations for practice. These midwives were also older and more experienced, although experience was not statistically related. There are several possible explanations for this relationship. It may be that those with higher caseloads are very busy, so neglect to refer when they should, or that they have a level of skill and expertise that facilitates more appropriate referral. In this study expertise was represented by years of practice and was associated with caseload but not with the rates of referral. Years of experience however cannot be regarded as a satisfactory measure of competence or confidence, and further research is needed to identify how and why midwives with higher 
caseloads make referrals. It is apparent that there is something about this particular group of midwives and the way they practice which would be important to explore.

It is within the medical rather than in the midwifery literature that the effect of caseloads on practice is addressed. In two studies, looking at whether delivery volume of family physicians had an impact on maternity outcomes both Janseen (2002) and LeFevre (1992) found that there was no difference in birth outcome, but that practitioners with small caseloads had a higher referral rate. Klein et al (1983) commented that the few studies that had been done of patient volume and outcome had shown variable effects, and concluded that there was no reason to restrict the practices of those with small caseloads, even though they referred more. Tilyard et al's (1988) study of New Zealand GPs also showed that GPs with smaller caseloads referred and transferred more often that those with higher caseloads. Their study also showed that referral practices differed according to whether the GPs lived in rural or urban areas. This contrasts with the findings of this study, where no differences were found in the practices and attitudes of rural midwives, and with Rosenblatt's (1985) study of maternity care in small New Zealand hospitals, who found that that there was no significant difference between rural and urban practitioners, or between caseload and birth outcome.

The only study found which addressed midwifery referral rates was by Wallace et al (1995). They conducted an observational study of midwife-medical staff interaction in a labour ward environment, and compared the referral rates of junior and senior midwives. They found that junior midwives referral rates were higher in complicated cases, and lower where cases had been designated as low risk. This is an indication that experienced midwives were more proficient in identifying where risk factors arose in those women who had been designated as low risk. Further research on the relationships between experience, expertise, caseload, referral and birth outcome is needed.

No studies were located which addressed the relationship between midwives' attitudes and referral practices. There were studies found in the medical literature. Rowlands and Lewis (2001), in their study of GPs' referral practices reported a wide variation in practitioners' referral rates in all areas of primary health including maternity care. 
They commented, "It is reasonable to assume that referral thresholds reflect both experiences in early training and the underlying personality of the doctor, including their assessment of and tolerance of 'risk"' (p29).

These authors were particularly interested in proposing strategies for reducing referral rates and proposed that the biggest effect would occur if the referral rates of those with 'average rates', i.e. the biggest proportion, were to be targeted. This is, of course assuming that the referral rates are too high. They did conclude with the comment, "Those in primary care responsible for controlling budgets and commissioning healthcare must approach reduction in referral activity with caution until more is known about the factors underlying this fascinating but frustrating facet of healthcare" (p 29).

O'Donnell's (2000) comprehensive literature review on referral rate variation among GPs confirmed the existence of large and unexplained variation in referral practices. She found that patient characteristics accounted for less that $40 \%$ of the variation, and GP characteristics less than $10 \%$. No one variable or group of variables was a strong predictor of referral, which raised the question as to whether or not variation in referral practices is a problem. She did however conclude, "Intrinsic psychological variables, such as GPs' willingness to take risks, their tolerance of uncertainty or their perception of the frequency with which serious disease occurs, are also important" ( $p$ 469).

Both the Rowland and the O'Donnell studies discussed the relationship between attitude (in particular their attitudes to risk) and referral rates, but neither was able to clearly establish one. My study also sought to investigate this but found no statistically significant association between any of the attitudinal variables constructed, including attitudes to risk management and the medico-legal environment, with referral rate. This study is very much a first stage investigation into New Zealand midwives' views about this topic. Further research using different approaches may help to enlighten this fascinating but frustrating topic. The focus group study later in this thesis begins to do this. 


\section{Birth outcome and referral}

Another way in which patterns of referral might be evaluated is to assess the birth outcomes of the women for whom the midwives cared. One of the central concerns which led to this study is the rising rate of intervention, particularly the caesarean section rate. Although it is acknowledged that the decision for caesarean section is not one that the LMC midwife makes on her own, she is however involved in the assessments and discussions leading to it. The referral for obstetric consultation is a key action in the progression towards caesarean section. This study sought to discover whether there were any variables, including referral rate, which were related to the caesarean section rate.

The women in this study had a caesarean section rate of $11.5 \%$. The national rate for women who had booked that year with LMC midwives was 16.6\% (New Zealand Health Information Service, 2003, p122) [NB Nineteen percent of the national data was missing from this figure]. This rate of caesarean section is not high and is an indication that midwifery-led care may well have an impact on intervention rates, despite the continued climb in the national rate. Further research on the case-mix of the women cared for by midwives as compared with doctors is needed to establish whether this is in fact the case. Studies have been done which demonstrated that midwives who care for 'low-risk' women achieve a higher proportion of normal deliveries than obstetricians who care for 'low-risk' women (Cheyne, Turnbull, \& Lunan, 1995; Davis, Reidmann, \& Sapiro, 1994; Giles et al., 1992; Janseen et al., 2002; Oakley, Murray, \& Murtland, 1996; Rosenblatt, Dobie, \& Hart, 1997). This study also indicates that midwives may have better outcomes, when they care for higher risk women. Rowley et al (1995) in their Australian study of midwifery-led care, which included high risk women, also found this to be the case. This is an area ripe for further research.

Further analysis of the caesarean section rate of the midwives in the study revealed that an increased rate of caesarean section was associated with increased rate of referral. This does not mean the more referrals, the more caesareans, as this would be expected. It means that midwives who refer more of their women as a percentage have a higher rate of caesareans in their practice, an important finding in this study. 
There will be some women who, if they had been cared for by midwives with lower referral rates would not have had caesareans. It also says something about what might happen to women once they are referred, i.e. once they have been considered as 'at risk' enough to require obstetric advice. No other variables in the study, including both the demography and the attitudes of the midwives, showed any association with the rate of caesarean section.

The perinatal death rate of this group of midwives was 6.1 per thousand as compared with the national perinatal death rate of 10.2 per thousand in that year (New Zealand Health Information Service, 2003). Given the different but unknown case-mix between midwives and obstetricians and limitations of data size with this small number, it is not possible to make any definitive comment.

\section{Choosing the place of delivery}

There are no recommendations within the New Zealand referral guidelines about the place of delivery. New Zealand women are free to choose, availability permitting, either a home birth, delivery in a primary service or in a secondary/tertiary service. In the year of this study, $43 \%$ of women who delivered in hospital did so in tertiary hospitals, $42 \%$ in secondary hospitals, and only $15 \%$ in primary birthing units (New Zealand Health Information Service, 2003). Apart from the births in the primary units, these figures do not represent the degree of complexity of these women, as all secondary and tertiary units also support primary maternity care. For example between $85 \%$ and $92 \%$ of women who delivered in tertiary maternity units in 2000 had a Patient Clinical Complexity Level of 0 (New Zealand Health Information Service, 2003). This score is related to inpatient diagnoses, not referral criteria. An unknown number of women in this year delivered at home. This study revealed that only $35 \%$ of women required obstetric input. That so many of them gave birth in secondary or tertiary facilities must be of concern in terms of possible unnecessary intervention. There is a considerable body of research illustrating that women who book to deliver in primary maternity services have lower rates of intervention (Klein, Spence, Kaczorowski, Kelly, \& Grzzybowski, 2002; LeFevre, 1992; Rosenblatt et al., 1985; Tew, 1985). Mead and Kornbrot (2004), in a comparative study of UK midwives working in high intervention units and those working in lower intervention units found that midwives in all the units over-estimated risk but that those in higher 
risk units did so more often. Carroll, Reid, Ruderman and Murray (1991) conducted a chart review of 2365 'low-risk' women delivering in three hospitals, one of which was a low risk centre. They too found that that caring for low-risk patients in a highrisk care environment was associated with a higher intervention rate. Van der Hulst, van Teijlingen, Bonsel, Eskes and Bleker (2004), in their study of 'low risk' Dutch women found that multiparous women who had opted for a hospital birth were more likely to experience consultation and referral than those who had opted for a home birth. Of all referred multiparous women, those who had planned a home birth had fewer interventions. They concluded that the impact of that choice resulted in fewer interventions, even when referral and transfer of care occurred. There seems therefore to be something about the attitude of women which affects intervention rates, even when the women are referred. Given the conclusions of these research reports and the findings of this study, that higher rates of referral are associated with higher rates of caesarean section, there seems to be something important about keeping women away from a 'high risk' environment, and about keeping referral rates as low as possible.

Although not stated explicitly in the New Zealand referral guidelines, decisions regarding the appropriateness of the woman's choice of birthplace are made at any stage of the pregnancy, from the first booking to during the labour. A unique aspect of New Zealand midwifery is the fact that many midwives provide care across all spectrums of the maternity service. They care for women giving birth at home, in primary birthing units and they also use secondary and tertiary facilities. Many cross these boundaries. Where there is a change in the planned place of delivery, usually from a primary unit to a secondary service, midwives commonly follow the woman and continue to provide care. Although the degree to which midwives cross these boundaries was not directly assessed in this study, the level at which overall care is continued when referral and transfer of care occurs supports this assertion.

Although the woman is free, within the boundaries of her risk status, to choose her place of delivery, questions must be asked regarding the part played by the midwife in this decision. That so many low risk women in New Zealand are delivering in secondary and tertiary hospitals must be a factor not only of women's preference but also of midwives' preferences. Midwives' perceptions of managing risk outside secondary or tertiary hospitals are worth further investigation. Hunter (2003) 
conducted a study of New Zealand midwives who worked in both primary and secondary facilities, looking at the different ways that midwives practice in small maternity units as opposed to obstetric hospitals. It showed that midwives felt they practised differently in the different contexts. In small units they felt they could practice 'real midwifery' but when they worked in the bigger obstetric units they felt pressured to use the technology, to control the noise and to watch the clock.

Given the research cited above, it is of concern that a minority of New Zealand women are giving birth at home or in primary maternity facilities. It has been identified as an important factor in attempts to reduce the rising intervention rate, but has yet to be actively addressed by policy makers (Pairman \& Guilliland, 2003). If primary, birth centre care is to be developed however, it is important to be wary of over zealous risk screening and high referral and transfer rates. A common discourse in the literature was an implied acceptance that only 'low risk' women are suitable for midwifery-led care. It would be important to continue to support midwifery-led care with appropriate obstetric input for those women considered to be too 'high risk' to birth in primary birth units.

\section{Consultation and continuing care}

Within the New Zealand context, it is during the referral for obstetric consultation that the appropriateness of continued midwifery responsibility is considered. This is after the woman has already chosen and developed some degree of relationship with her midwife. New Zealand women do not expect to be screened and expect that they will be part of any decision regarding continued midwifery involvement, once pathology or risk characteristics are identified (Health Funding Authority Maternity Project Team, 1997). In many areas it is expected that the midwife will continue care and contact with the women she has booked. The results of this study, that $57 \%$ of the referrals for consultation did not result in transfer of responsibility and that in $73 \%$ of cases where transfer occurred the midwife continued to provide care, are a strong indication of the continued involvement of New Zealand midwives in caring for, and staying with, 'at risk' women.

There is one sentence in the New Zealand referral guidelines which is pivotal in this particular aspect of the research. "There are some particular circumstances, for 
example twins, where clinically the specialist needs to be responsible for care but the ongoing involvement of the primary practitioner is very important" (Ministry of Health, 2002, p31).

This statement is open to considerable interpretation and relies heavily on the goodwill and trust between midwife and obstetrician. The findings of this study, that where clinical responsibility has been transferred $73 \%$ of the women continued care with their midwife to some extent, indicate that some particular circumstances are in actual fact most circumstances. Policy would appear to be at odds with practice. In general, it appears that most services are supportive of the midwives continuing care. Seventy two percent of the midwives in this study reported that obstetricians supported continuity of midwifery care and $64 \%$ felt that there was excellent collaboration between primary and secondary services. For the remaining midwives the situation was more difficult. Where obstetricians are not supportive of continuity of midwifery care they can, and have, refused to collaborate with the midwife LMC. The midwives in this study, no longer the LMC, continued to provide care for 108 women $(18 \%$ of women who had had care transferred) without payment. Such was the commitment to being 'with' the woman throughout her childbearing. This is further supported by the fact that only $17 \%$ of the midwives in this study disagreed to any extent with the statement that they should care for low risk women only, and also by the high proportion of midwives who attended the first antenatal consultation.

In order to continue care into the secondary environment, New Zealand midwives have access to secondary and tertiary units. Skills such as augmentation of labour and maintenance of epidural anaesthesia are accepted parts of their scope of practice. This makes midwifery in New Zealand quite unique. Independent practitioners based in the community, and not employed by the hospital, provide both primary and, in collaboration with obstetricians, secondary care. What adds to its uniqueness is the fact that the majority of New Zealand women have this service. It is not an exception, nor is it part of an experimental study. It is now mainstream and 'real world' and it is an important finding of this research. It could also be considered as a very large natural experiment, and midwives from other countries have been interested to watch its development and its outcomes. 
The degree to which midwives continued care for women who had planned to give birth in primary facilities and required transfer to secondary obstetric facilities was not assessed in this study, and is an important area for further investigation. It was also not possible to compare New Zealand midwives' practice of continuing care with midwives' practice in other countries. None of the studies cited above provided any detail about referrals for consultation that did not result in transfer of care, nor did they discuss the extent to which the midwife continued care once the responsibility for care had been transferred. This issue was, I thought, conspicuous by its absence.

\section{Using the referral guidelines}

The referral guidelines that New Zealand midwives use differ fundamentally from risk screening tools in both approach and outcome, and more closely resemble the alternative models which emphasise community-based continuity of care within integrated reproductive health services, and with early and appropriate management of obstetric complications. The central focus of the New Zealand maternity service is the childbearing woman, rather than the needs of the practitioners or of the institutions. The preamble to the referral guidelines state that they are to be used to facilitate consultation and integration of care and that there may be flexibility in their use. They depend on sound clinical judgement and informed consent. They also recognise that in some circumstances where responsibility for care is transferred to the obstetrician, ongoing involvement of the primary practitioner may be important. Where a consultation occurs it must involve a three-way discussion between the specialist, the LMC, and the woman about ongoing clinical roles and responsibilities (Health Funding Authority Maternity Project Team, 1997). They are intended to promote collaboration between primary and secondary care, as opposed to creating a cut-off point between them. They do not involve any totalling of numerical ratings to give an overall risk score.

These guidelines were developed in a collaborative way between representatives of midwives, obstetricians and general practitioners. As indicated in this study, midwives found them a useful tool, although there was some ambivalence about whether they thought the guidelines required referral more often than they thought necessary. Not surprisingly, the midwives who were most positively inclined towards 
the guidelines were those who had positive collaborative relationships with the secondary service. Those midwives who were less likely to find the guidelines useful, and who thought that they were required to refer more often than necessary, also reported a lack of support from their secondary service. No research has been undertaken as to the effectiveness of the guidelines, but as this study illustrates, there does seem to be reasonably widespread acceptance of their use. Certainly in medicolegal terms, they are used as a marker of appropriate midwifery practice. Whether their raison d'etre is political or clinical, the effect they have on the women and whether they are useful or not, remains to be evaluated. Given the previous research on risk screening so far, they are unlikely to show positive predictive value but may have other positive effects such as providing some parameters for midwifery practice and facilitating clarity of collaborative processes.

\section{Referral guidelines in other countries}

An exploration of the international literature and on-line resources revealed that there is little published material on describing or interpreting referral guidelines. Haertsh, Campbell and Sanson-Fisher (1999), in their comparison of seven prenatal clinical practice guidelines included only two based on scientific evidence. All were dominated by biomedical and technological approaches. The New Zealand guidelines also reflect this. Only biomedical indications are included and although the document states, "This document provides guidelines for best practice based on expert opinion and available evidence" (Ministry of Health, 2002, p31), there is no scientific evidence provided.

I obtained a variety of referral/transfer guidelines while on a study tour of the United Kingdom, Ireland, Denmark and the Netherlands. The types of guidelines obtained varied, from detailed and explicit national referral guidelines to single page problem lists created in individual maternity units. The Netherlands (National Health Insurance Board of the Netherlands, 1998), New Zealand (Ministry of Health, 2002), British Columbia (College of Midwives of British Columbia, 1997) and Australia (Australian College of Midwives, 2004) have guidelines which were very similar. These guidelines were developed collaboratively between practitioners. They provide lists of conditions for each segment of the childbearing process and graded recommendations for referral for consultation. 
I was unable to find national guidelines for midwifery referral in either United Kingdom or the United States, but guidelines were developed in smaller areas taking into account regional differences. In the case of the United Kingdom, these guidelines appear to be developed within each National Health Service Trust. The referral guidelines I obtained when in the United Kingdom were variable. Some still had a totalled numerical risk scoring system and others provided a list of conditions where transfer from the primary to the secondary service was mandatory. All related to assessing suitability for community care in a primary birth setting and the criteria listed were contraindications for community care. The listed conditions in all the guidelines viewed were very similar.

Given the lack of scientific evidence available on who needs consultation and who needs referral, guidelines related to referral are open to abuse. They have the potential to be used as political instruments of control over practitioners who have a less powerful position and can be used as an instrument of surveillance to control practitioners who are working outside normally accepted parameters of care (Davenport, 2000). In this way they have the potential to be too restrictive, limiting creative or innovative practice and can result in a single worldview being presented. They can also result in rates of referral which are too high (O'Donnell, 2000). For example when Haertsh, Campbell and Sanson-Fisher (1999) compared the Scottish and German maternity referral guidelines, they found that there were many more women in the German guidelines (71\%) who were categorised as high risk.

The discussions around the development of the New Zealand referral guidelines were not easy and considerable compromise was needed (personal communication CEO NZCOM). They were a pragmatic exercise in interdisciplinary collaboration rather than an exercise in evidence based decision-making. Interestingly the most recent review of these guidelines held in 2001 was unsuccessful, as the GPs refused to participate. They did not wish to be covered by the same guidelines ast applied to midwives. At this time therefore they remain unreviewed. 


\section{Reasons for referral - 'taking too long'}

Having identified that the case-mix of the midwives in this study includes women with a variety of risk profiles, some further discussion about the reasons for referral is needed, particularly in relation to referral being an expression of risk and its management. In order to do this the two reasons for referral - 'prolonged pregnancy' and 'lack of progress in labour' - are identified as being of particular interest. The interpretation of these rates will depend on which lens is being used to view the issue. One might consider that the high rates of referral for these reasons illustrates that midwives' referral practices are appropriate. The midwives seek assistance in a timely manner in line with current practice norms.

However, given current concerns regarding increased rates of intervention, these reasons require more in-depth analysis for a number of reasons. In this study they are examples of referral where no pathology or co-morbidity exists; they represent risk management in its pure form. 'Taking too long' either in the pregnancy or in the labour are the most common single reason for referral and their relationship with operative delivery is statistically significant. The midwives who made these referrals had higher overall referral rates and higher caseloads, although, given that most of the midwives (244 and 277 respectively) made referrals for these reasons this finding is of minimal importance. These high rates of referral for prolonged pregnancy and lack of progress in labour are reflected in other studies (Hundley et al., 1994; Kean et al., 1996; MacVicar et al., 1993; Radomsky, 1995; Reid et al., 1995; Smit et al., 1997; Wallace et al., 1995), and have been identified as one of the main causes of increased intervention (Alexander, McIntire, \& Leveno, 2001; Duff \& Sinclair, 2000; Maslow \& Sweeney, 2000). It seems that a large number of women 'take too long'. Particularly telling are referral statistics from the Netherlands. From 1989 to 1993 the number of referrals for every maternity complication rose, but the number of referrals for insufficient progress in both first and second stages of labour rose the most (Pasveer \& Akrich, 2001).

Although it is beyond the scope of this study to provide a detailed critique of routine induction at 41 weeks or about what might be considered too long a labour, critiques of these can be found in the work of Menticolou and Hall (2002); Alexander. McIntire 
and Levene (2001), Albers (1999), Gifford, Mortal et al (2000) and Zhang, Troendle and Yancey (2002). Menticolou and Hall analysed Crowley's (2001) meta-analysis of RCTs related to routine induction at 41 weeks, and commented that 1000 routine inductions at 41 weeks, where there was with no other clinical indication may save the life of one baby. They proposed that this policy has significant resource consequences and other unintended and undesirable consequences, some of which remain unresearched. Albers (1999) and Zhang et al (2002) in their studies assessing the duration of normal labour in healthy women, concluded that labour lasts longer than many clinicians expect and that the pattern of labour progression differs considerably from the Friedman curve. Further support for the argument, that fewer women are 'taking too long' than are thought to, is found in the study of Gifford, Morton et al (2000), who reviewed the documentation of 733 women with nonbreech, term pregnancies, who had unplanned caesarean sections. They reported that $16 \%$ of women who had had a caesarean section for lack of progress in labour were still in the latent phase and that $36 \%$ of women who had a caesarean section for prolonged second stage had, in fact, not had a prolonged second stage, according to the American College of Obstetricians and Gynecologists' criteria. It would seem that 'too long' is getting shorter.

Despite the inability of this study to accurately compare the rates of referrals for these reasons with those found in other studies, the fact that the midwives in this study had such high rates of referrals for these reasons is of particular concern. This issue is a reflection of the tension between different approaches towards the concept of 'normal' and its relationship with risk as discussed in Chapter 2. The findings of this survey, that so many referrals are made for prolonged pregnancy and lack of progress in labour and that midwives felt that their pattern of referral was appropriate to the needs of the women they cared for, is an indication that midwives may be captured by the techno-rational risk discourse, often referred to as 'the medical model'. The focus group studies, later in this report, also address this issue and highlight it as of being of primary concern. Other studies of midwives have also found this to be the case (Johanson, Newburn, \& Macfarlane, 2002; Kirkham, 1999; Olsson, Jansson, \& Norberg, 2000). Given that midwifery-led care is the dominant model of maternity care in New Zealand, one might have surmised that there would be a decrease in medical hegemony, reflected in a greater tolerance for longer pregnancies and 
labours. The fact that all but one of the guidelines located for this study, including the New Zealand referral guidelines, recommend referral and induction at 41 weeks and that the use of the partogram and the Freidman labour curve are still usual practice, would belie this.

These findings also support Beck's (1999) contention that we live in a 'risk society'. Maternity care is not alone in its techno-rational approach, and ideas of managing and controlling risk abound. That we would induce the labour of 999 healthy women in order to save the life of one baby with little regard to either resultant morbidity or even mortality, reflects the paradox of living in this 'risk society'. In order to control risk we create risk. The contention that replacing medically-led care with midwiferyled care would directly lead to improved outcomes for women, belies the complex processes of authoritative knowledge and the socio-cultural 'risk' context in which care is provided.

\section{The medico-legal environment}

Beck (1999) proposes that in a 'risk society' there is a growing perception that negative outcomes should be able to be prevented and that the expert must be responsible for them. A negative outcome is seen as being caused by inadequate risk management. These risk management procedures originated in the fallout from litigation, which has seen the development of another category of risk - risk to the practitioner (Blandford \& Smyth, 2002). Without doubt they are inextricably linked. Beck's contention that the techno-rational approach of modernity has led to the risk society is supported by Johanson, Newburn and Macfarlane (2002), who propose that a medicalised approach is associated with constraints especially, the "name, shame and blame' culture. They cite the National Health Service in the United Kingdom as an example where, in 2001, the litigation bill was $£ 2.6$ billion, double what it had been in 1997 . Seventy percent of the claims related to obstetrics and $99 \%$ of these obstetric claims relate to 'failure to intervene' or 'delay in intervention'.

In New Zealand we have been shielded from the worst effects of these legal actions by the Accident Compensation Corporation, but are not shielded from public and 
professional accountability. Risk management is still a core issue at both an institutional and individual level.

The midwives in this study agreed strongly that risk management was an important part of their practice. Most midwives had negative attitudes to the medico-legal environment. Only 28\% thought that medico-legal issues did not restrict their practice and $25 \%$ disagreed with the statement that the medico-legal environment made childbirth safer for women. For the midwife there is an added concern related to the medico-legal environment. When a complaint is laid about midwifery practice an obstetrician is usually given the opportunity to provide comment. Given the medical dominance of birth it will be the medical definition of acceptable practice that will be the one to hold most authority. There are in New Zealand some indications that this may be changing, with the increased place that midwives are playing as expert witnesses. Their advice however can be overturned by medicine's which is seen as having the authoritative voice. The impact of the medico-legal environment on midwives' lives is explored in greater depth in the focus group study later in this thesis.

\section{Summary}

This survey has described the practices and processes of referral for obstetric consultation of New Zealand LMC midwives as an expression of risk management. It has also sought to gain some information about their attitudes to these processes and to the risk context in which they are practised. Both the respondents to this total population survey and the women for whom they cared were representative of the total populations from which they were selected. This survey is the extensive or quantitative aspect of the research. As little has been known about this issue, the survey has taken a broad approach and has provided valuable information on which further research may be based.

New Zealand midwives have developed a unique model of care, which is focused primarily on the connection that they have with women. This is reflected both by the extent to which they offer their services to all women, not just to the 'low risk', and by the extent to which the midwives continue to provide care when obstetric input is 
required. They believe strongly that this should be the case. Only $3.5 \%$ of all the women for whom the midwives in this study were LMC did not continue care with her to some extent. This model of care differs markedly from those found in the studies of midwifery-led care in other countries where care is concentrated on those who are considered 'low risk' with a focus on providing care in the primary care environment. As this study has shown, this latter model risks excluding a large number of women from continuous midwifery care but does have the advantage of improving the birth outcomes of those women, albeit a minority, who plan to birth outside of secondary facilities. The New Zealand model also sees a minority of women planning to give birth at home or in primary units, environments where risk is managed very differently. It would seem to be important for New Zealand midwives to support and encourage women to deliver away from an environment where an interventionist approach dominates and where women who are deemed as low risk are treated the same as those with higher risk status. These two models of midwifery care have quite different foci, one on the choice of caregiver and the other on the place of birth. Over the last 14 years in New Zealand we have focused on developing the LMC midwifery role. It seems an appropriate time now to concentrate our efforts on providing appropriate environments in which women may give birth.

This survey also sought to assess the appropriateness of New Zealand midwives' referral practices, particularly the rate of referral, and to highlight any areas of concern where intervention or further research may be needed. The referral rate was $35 \%$. Given the limitations of national and international recommendations and the wide variations in practice patterns of midwives in other countries, any conclusion regarding the appropriateness of this rate is difficult. Studies assessing variation in referral rates in the medical literature confirm wide variation in referral rates and the problematic nature of assessing its appropriateness. The fact that referral rates in this study do not vary according to the demographic characteristics, practice patterns (except caseload) or attitudinal characteristics makes it very consistent across the midwifery population, and indicates that there is little cause for concern in these areas. The three findings within this study which are of interest however, are the relationship between referral rate and caseload, and the relationship between referral rate and caesarean section. The third finding of particular interest was the number of 
referrals for prolonged pregnancy and for lack of progress in labour, both expressions of risk management associated with 'taking too long'.

What has become apparent in this survey, is that the practice of midwifery in New Zealand is both defined and not defined by risk. In some essence the midwife is present because there is risk. She provides care both despite it and because of it. She does not seek to avoid it. Risk, in the New Zealand context, does not define the parameters of care, the woman does. Within this context the midwife has great freedom to provide care autonomously, yet at the same time is constrained by the realities of the risk that she is there to manage and the realities of a socio-cultural risk environment in which techno-rational approaches to birth dominate. This dilemma is reflected in the literature relating to the problematic nature of risk screening guidelines.

This study indicates that New Zealand midwives are not risk averse, in that they do not seek to avoid the care of women with indications of increased risk. Yet they also believe that risk management is a very important part of their practice. Despite some concerns about referrals for women who 'take too long', their referral rates do not appear to be excessive. Many have positive relationships with the secondary services. The following focus group discussions explore these practices and attitudes further, in order to come to an understanding of how midwives resolve these issues, and make sense of midwifery practice in a risk environment. 


\section{Chapter 7: Focus Groups - Design and Process}

\section{Introduction}

The three previous chapters have presented the design, the results and the first level interpretation of the national survey. This was the 'extensive' or quantitative aspect of the study. The survey has described the LMC midwifery workforce in New Zealand and has also provided a description of their referral for obstetric consultation practices. It continued by assessing, quantitatively, some of their attitudes towards the primary/secondary interface, the medico-legal environment, and risk management.

This chapter provides the design detail and process of the 'intensive' or qualitative process of the research. This further inquiry was undertaken in order to provide a more in depth understanding of the midwives' attitudes and practices. Focus groups were the chosen method and the rationale for choosing this method has been presented in Chapter 3. This chapter describes how the groups and the participants were selected and covers issues of facilitation and group process. It discusses the pertinent ethical issues. It then provides a detailed explanation of the way the data were analysed.

The group process findings and emergent coding categories and themes are included in this chapter alongside the description of how the analysis was undertaken. The decision to include this level of analysis at this stage is a pragmatic one as reading coding on its own, separate from either the description of the analysis process or without some interpretation is both uninteresting and inefficient. Given the mixed methods approach of this study and the need to provide comprehensive interpretation incorporating both research approaches, it is more appropriate to include the coding and group process findings alongside the design. Including this first level analysis in this chapter also provides explicit evidence of the thoroughness of the analysis, contributing to an understanding of the trustworthiness and integrity of the research. 


\section{Aims of the focus groups}

There are different possible uses for focus groups, so the design of a group is very dependent on what the group is intended for. Focus groups can be composed of different categories of members and be used for a diverse number of tasks such as brainstorming, consensus reaching or ranking exercises (Kitzinger \& Barbour, 1999). This will impact significantly on both the design of the group and the way in which the data are analysed. Clear aims facilitate appropriate process and effective, valid interpretation. The specific aims of the focus groups in relation to the research question were:

- To provide contextualised, qualitative data in order to reflect the depth and complexity of the research question

- To hear voices of the midwives from as diverse perspectives as possible

- To undertake the discussion in an environment where midwives could share perspectives with each other and where the perspectives of the midwives, not the researcher could be central

- To inform the development of a theoretical approach to midwifery as it is practised in New Zealand and as it relates to the risk environment

There was no intention in these groups to attempt to reach consensus. Nor was there any intention to delve deeply into individual midwives' experiences. Had I intended to do that individual interviews would have been preferable. Instead the data produced were analysed according to the group process and coded categories.

\section{The participants}

Considerations in planning the focus groups were related both to the number and locations of the groups and to the number and choice of participants for the groups.

\section{Selecting the regions}

The groups were selected according to characteristics that would provide the most contrasting views and that were most related to the theoretical issues involved (Knodel, 1993). I had already identified in the planning of the survey what the key areas of difference were likely to be and I decided to continue this approach in the 
design of the focus groups. This decision aimed to maximise variation in voice and practice experience. In order to do this the locations of the focus groups were chosen to reflect different practice environments. The four criteria for choosing the areas where focus groups would take place were whether they were rural or urban, whether they were in the North or South Island, whether they had secondary or tertiary maternity units in their area, and the degree to which they had positive or negative relationships with the secondary care services. These were the characteristics over which I had control. I planned to undertake six focus groups as this would enable cover of all the combinations of the 'break' characteristics I had identified. All of these characteristics were represented in more than one area (Table 7.1). The selection of the areas was informed by the survey findings, and supported by the National Maternity Services Manager whose advice I sought. The final choice and order also had a pragmatic turn, as timing, costs, willing participants and visiting for other reasons also impacted on the final decision.

\section{Recruiting the group members}

Once the regions had been selected, potential participants were then contacted. The first level of contact was made using the information returned with the completed questionnaire. A tear off section, asking for contact details of the participant was attached to the information sheet that had been included with the questionnaire (Appendix C). The participants returned this form if they wished to be part of a focus group. After the regions for group discussion were selected the participants from those regions who had indicated an interest in participating were contacted. This led to enough participants in two of the regions. In the other four regions I recruited further participants by requesting local contacts or local College of Midwife coordinators to ask for participants on my behalf. This proved to be successful in all but one region, where only one participant came to the meeting. Without exception the midwives I made contact with were enthusiastic in supporting the research. One of the midwives stated "We've been talking about this for ages. It's about time someone did something with it". 
Table 7.1 Characteristics of regions selected for focus group interviews

\begin{tabular}{llllll}
\hline $\begin{array}{l}\text { Group } \\
\text { number }\end{array}$ & Rural/urban & $\begin{array}{l}\text { Secondary/tertiary } \\
\text { facility to which } \\
\text { referrals made }\end{array}$ & $\begin{array}{l}\text { Number of } \\
\text { participants }\end{array}$ & $\begin{array}{l}\text { North } \\
\text { Island } \\
\text { /South } \\
\text { Island }\end{array}$ & $\begin{array}{l}\text { Relationship } \\
\text { with } \\
\text { secondary } \\
\text { services }\end{array}$ \\
\hline 1 & Urban & Tertiary & 4 & South & $\begin{array}{l}\text { Medium to } \\
\text { negative }\end{array}$ \\
2 & Urban & Tertiary & 4 & North & $\begin{array}{l}\text { Medium to } \\
\text { positive }\end{array}$ \\
3 & Rural & Secondary & 7 & North & $\begin{array}{l}\text { Positive } \\
4\end{array}$ \\
Rural & $\begin{array}{l}\text { Secondary } \\
\text { Secondary }\end{array}$ & 5 & $1 *$ & $\begin{array}{l}\text { North } \\
\text { South }\end{array}$ & $\begin{array}{l}\text { Negative } \\
\text { Positive }\end{array}$ \\
6 & Urban & $\begin{array}{l}\text { North } \\
\text { Secondary }\end{array}$ & 6 & Negative \\
\hline
\end{tabular}

*Due to a communication error only one participant came to the meeting. It was not possible to attempt another meeting. Due to the quality of the discussion and the fact that it occurred in the only urban area what had reported positive relationships it was decided to include the data in the analysis.

The decision about the number of participants to invite to the group needed to be carefully considered. What I was quite sure of was that there would be a considerable number of midwives who would not attend even after having agreed to do so. The life style of a midwife is such that births and general busyness often gets in the way of such occasions. There was also a disconcerting number who said that they would 'try and get there'. Because of this I felt reasonably confident to attempt to recruit a larger number than would be comfortable. There are a variety of recommendations for the number of participants to include in focus groups. Morgan (1998) for example, recommends 6-10 and Langford and McDonagh (2003) recommend 5-12. Kitzinger and Barbour (1999) suggest that in social science research, smaller numbers are more appropriate. Small numbers in a group enable each member a greater opportunity to talk ensuring a better quality and depth of the data and possibly greater comfort in the groups (Carey, 1994). Where possible I invited 10 midwives to each meeting. From the initial planning stages of the research it was likely that the number of group members would vary. The group process therefore needed to be flexible enough to deal with this.

I was not able to control for the characteristics of the midwives in the groups. In the event each group had different selections of midwives (Table 7.2). In all but one of the groups the midwives knew each other and some were working in the same group practice. There was a good spread both of experience and caseload, with a small 
number who were in the process of either returning to hospital employment or taking a break from practice.

Table 7.2 Characteristics of focus group participants

\begin{tabular}{lllll}
\hline Experience & Full caseload & Small caseload & $\begin{array}{l}\text { Changing } \\
\text { practice }\end{array}$ & Total \\
\hline $\begin{array}{l}4 \text { or more yrs } \\
\text { experience }\end{array}$ & 12 & 2 & 2 & 16 \\
$\begin{array}{l}\text { Less than 4yrs } \\
\text { experience }\end{array}$ & 5 & 4 & 2 & 11 \\
\hline Total & 17 & 6 & 4 & 27 \\
\hline
\end{tabular}

These groups then reflect variety in a number of ways: in urban and rural experience, in secondary and tertiary maternity support with varied intervention rates, and in the nature of the relationships with the secondary maternity service. The decision to conduct six focus groups was not seen as necessarily a finite number. Pragmatically it was thought that the competing or mixture of voices would all get a chance to be heard in these six groups. There was opportunity to continue with further groups if it was felt that more experiences or viewpoints needed to be heard. In the event this did not happen. There was a general sense of 'saturation' in the voices heard.

\section{Facilitation}

Much of the literature on focus groups stresses that the facilitation of a group process requires careful management and that the role of the facilitator is crucial (Carey, 1994; Fern; Knodel, 1993; Krueger, 1994; Morgan, 1998; Sim, 1998; Stewart \& Shamdasani, 1990; Wilkinson, 1999). Sincerity, humour, flexibility and a good memory are important. Facilitators must have the ability to listen and to detach themselves from the issue in such a way that they do not preach, teach or correct (Wolff, Knodel, \& Sittitrai, 1993) and not to be heavy handed with the interventions (Kitzinger \& Barbour, 1999). I was however encouraged by Morgan and Krueger (1993), who commented that facilitators of focus groups do not necessarily need to have highly developed professional moderation skills. They stated that it is both more feasible and preferable to have a moderator with knowledge of the topic. They did note that the moderator is a data collection instrument so must be prepared, attentive and skilful. 
I decided that I would facilitate the groups myself. As a lecturer in a postgraduate school I have considerable experience in leading and developing discussion. I wanted to ensure that the discussion was managed in a way that was consistent with my research interests and goals (Morgan \& Krueger, 1993). It would have been very difficult to find another skilled facilitator in each of the areas I visited and then to be able to prepare them in enough depth prior to each group.

The literature on the management of focus groups often recommends having an assistant moderator (Krueger, 1998). The functions of this person are to assist with the smooth running of the group, to take notes of who was speaking when and to note any group interactions that might not be recorded on the tape such as non verbal communication or those instances where more than one person is speaking. They might also be able to reflect immediately after the group with the facilitator and highlight impressions and comments about any discussion. For the first two groups I had the assistance of two midwives who were lecturers at the local midwifery schools. They did not participate in the discussion but did do some note taking. In the event, what they did was write down the first initial and first few words of each speaker in order to assist in transcribing. However I was able to manage this process myself quite easily. The next two groups were in rural areas where I wanted all interested midwives to be able to be part of the group, not observing, so I made the decision not to have an assistant. The groups were in general not large and I found that I was able to capture the process well on my own. I think that familiarity with the lives of midwives and my facilitation skills precluded the need for extra assistance at this time. Research assistance of this type does require some expertise and preparation and as the focus groups were spread around the country it was impractical to be able to do this. I therefore did not have assistance for four of the discussions.

This was, though my first experience of leading focus group research. For the first group I was quite nervous and over-organised. I had nametags for each midwife (even though they all new each other) and was careful to give the assistant clear instructions about her role. I relaxed considerably over the time of the groups and was, I think more able to be responsive to comments and to be more permissive about letting the conversations develop and letting the midwives take the conversation 
where they felt it needed to go, before interrupting. In general I found the discussions lively, enjoyable and very interesting.

I was very aware of my own strong interest in the issue and where my own beliefs were sitting. It was important to consider what role I would take in the discussion to create an ambiance which would influence how the participants shared their information (Krueger, 1994). I positioned myself as a 'wondering' person, interested in the midwives' perspectives and practice experiences and keen for them to share these with me and with each other (Kitzinger \& Barbour, 1999). The midwives knew then, that it was an issue that we had in common. We could move the discussion quite quickly from the basic description of how things were to how they perceived them. I rarely added my own comments and where I did it was usually to reframe and interpret the comments before moving on to the next area of discussion. In general I had low moderator involvement (Morgan, 1988). One thing that I planned to do which proved worthwhile was to bring in the results of the national survey at the end of the discussion. The midwives were all keen to hear what I had found and offered lots of comments about what it might be interesting to find out from the data.

The principles of facilitation that I developed related to my own positioning in the research and to the aims of the study:

- I shared with the midwives who I was and why I was interested in the project

- Once the conversations began I interrupted only to get clarification, to ask a new question, to summarise what had been said or to encourage quieter members of the group to make comments

- I was flexible about how the conversations developed

- I moderated situations in which midwives expressed disagreement or when midwives became distressed

- When the discussion went completely off the topic I refocused it

- At the end of the discussion I shared some of my perspective and the preliminary descriptive result of the survey, asking the midwives what they would like me to investigate further. 


\section{The process of the discussion}

Participants were welcomed to the meeting. If they did not know each other they were introduced. At each group there was food and refreshments available (Carey, 1994). Consent forms were signed (Appendix D). As the focus group was being used to investigate an issue in some depth a semi-structured format was followed (Morgan \& Krueger, 1993). The question guideline was introduced in a flexible manner and was open ended and general (Knodel, 1993) (Table 7.3). I started the conversation with a description of my research and established ground rules for the discussion. These issues had also been covered in the Information Sheet (Appendix D). I then asked the midwives to introduce themselves and to give me some idea of their practice experience and their present practice patterns. I then opened the conversation up by asking them to tell me how the referral for secondary care services worked in their area and whether or not they found this worked well both for them and for the women. This proved to be a good place to start as it was an easy question to answer and enabled the midwives to relax about being on the tape. The rest of the discussion loosely followed the guide that I had prepared for the group. In many instances the midwives themselves raised the issues and answered the questions without my needing to. They arose spontaneously within the discussion, which confirmed my feeling that the questions were interconnected. There was some variation in this as different groups raised different issues that they felt were important.

Immediately following each group I listened to the tapes and wrote detailed notes of the meeting. These notes included a summary of the whole meeting, important first impressions of both the participants and the data. I noted anything in the discussion that I thought was particularly valuable. I wrote a critique of my facilitation and detailed what might be important to consider in subsequent groups. A transcriber entered the data into the computer. She signed a confidentiality agreement (Appendix E). NVivo computer software was used to manage the coding associated with the analysis. 


\section{Table 7.3 Focus group question guide}

Local referral for obstetric consultation patterns One of the things that I am very interested in is how midwives feel about the referral for consultation. How do you feel this is managed here in your area? What are some of the tensions and difficulties with referral? How is the interface between primary and secondary care dealt with here? What makes it work well? How would you like it to work?

Risk labelling What do you think is the best way of providing midwifery care for women "at risk"? How do you feel about women being labelled "at risk"? Is there an alternative to this?

Normal Midwives are said to be the specialists in the normal. Do you think LMC midwives should continue to provide care for women no longer normal? How do you decide what is no longer normal?

Referral guidelines Are they useful? What do you think women want?

Risk How do you communicate risk to the women you care for? Do you take risks? How do you decide about what risks to take? What do you do to minimise risk? Do you think you manage risk differently from obstetricians?

Medico-legal environment How do you think the medico-legal environment impacts of your practice? How does it feature in your daily decisions? E.g. documentation? Is anything different about the care you give to women in this environment? Do you refer more? How does it affect issues of choice and informed consent?

Risk society Risk seems to be a huge thing in our society and it impacts on everyone not only institutionally but in our own lives. What do you think is going on that might be causing this?

Summary and feedback, followed by a presentation of my survey results

\section{Ethical implications}

The main ethical implications of these focus groups were the maintenance of confidentiality and non-identifiability, and the protection of the participants' well being. To this end ground rules for the focus groups included a request that the participants would honour each other's perspective and experience. They were asked not to reveal who was in the group or anything talked about in the group. During the discussion I anticipated the possibility of any breach of these agreements and was attentive to the possibility of midwives' revealing details not appropriate in this setting. This did not happen in any of the groups. The participants had my contact 
details and those of my supervisor and were encouraged to make contact if there was anything of concern. None did. Consent forms were signed.

The names of the members of the group are confidential to the other members, my research supervisor and myself. The transcriber has signed a confidentiality agreement. The location of, and data from the groups was made anonymous after transcription. The tapes and original transcripts are kept in a secure cabinet and will not be listened to by anyone other than my research supervisor and myself. The transcriptions of the tapes were not returned to the participants, as this would have meant that they had written evidence of another's words. It was felt that to return only their segment of the discussion would have little meaning out of context. All the participants were happy with this. In general they did want to receive a summary of the research on completion. This research report is written in a way that neither the location of the groups, nor the members of the groups can be identified.

\section{Data analysis}

There is no one particular approach recommended for the analysis and interpretation of focus group data (Kitzinger \& Barbour, 1999) and there is considerable diversity about how this might be undertaken. One can take a more quantitative, computer based approach or go to the other extreme and undertake a critical discourse analysis, to mention just two. There is however a scarcity of instruction related to the analysis of focus group data especially as it relates to the extent and quality of the group interaction (Frankland \& Bloor, 1999). This group interaction may be the cause of any attitudinal consensus or strength of opinion rather than as a factor of individual opinion per se (Sim, 1998). A further problem with focus groups is how to analyse and present such rich but untidy and unstable pictures (Waterton \& Wynne, 1993). Some of these problems can be overcome by placing the members within their context and by acknowledging their experience of the issue (Merton, Fiske, \& Kendall, 1990). In order to throw light on the substantive issues within the rich complex data of a focus group, the process of the analysis and the relationship of the analysis to both the research question and to the methodological approach needs to be systematic and explicit. 


\section{A framework}

There were two principles that informed the way the focus group data in this study were analysed. The first related to the way I positioned myself within the research. I entered the research setting and analysed the data keeping in mind my own subjectivity -"This is how I see it. I wonder how others see it?" I wanted to be surprised and so looked for other interpretations. The second principle was related to the purpose of the research. This study aimed to explain how midwives deal with risk in practice and to provide a model of how midwives might make sense of working in a risk environment. I wanted this model to be both meaningful and challenging for practising midwives and useful and enlightening for policy makers and educators. I therefore needed to breach 'the theory-practice gap' (Morse, 1994).

The focus group data were first analysed at two levels. The first consisted of an holistic examination of each of the groups including discussion of the group interaction and what could be learned from this. In the second level of analysis each transcript was coded. The codes from each transcript were then merged and grouped into themes (Morse, 1994). The remainder of this chapter provides the detail of how this proceeded. In the next chapter, the final level of analysis is presented. This is where the holistic, group analysis and the coded themes were merged with the quantitative data and the relevant theoretical perspectives in order to develop a new theoretical model on how midwives deal with risk (Knodel, 1993). These three stages of the analysis represent the processes of induction, deduction, abduction and retroduction discussed by Danermark el al (1997).

\section{Group process}

Focus group data poses special challenges for data analysis due to both the social context in which they are set and to the interactions inherent in the process. The groups were viewed as " a site of constant negotiation, involving elements of the participants' and the researchers' identities, and an iterative shifting and resettling of positions within the group around the issues that arise in the discussion" (Waterton \& Wynne, 1993, p133). 
At this stage of the analysis the focus groups were not seen as a source of detailed 'themes' but as a source of interaction and all that went with it. The analysis looked at the associations between people, not data sets (Morse, 1994). The different ways of talking, such as telling stories, joking, accommodating others' opinions and shifting positions provided an important source of data (Waterton \& Wynne, 1993). The tapes therefore were firstly heard and analysed as a whole.

The analysis first referenced the group contexts, starting with the group as a whole rather than the individuals. Just as the participants in the groups were varied, so too were the groups, not only in locality but also in how they were constituted. They varied considerably. In each of the groups this different mix of members made detailed comparisons across groups inappropriate. What was more appropriate was the comparison of broad themes (Carey, 1994). Within the groups, opinions were acknowledged as being provided in agreement or disagreement with others (Kitzinger \& Barbour, 1999).

\section{Group One}

This group was held in the evening in the room of a local house that was used by the midwives for antenatal visits and classes. This was an urban region and the nearest hospital provided care to a tertiary level. The relationships between the independent midwives and the secondary maternity service were recorded in the survey as being of medium to low quality.

The conversation was vibrant, engaging and enjoyable. It seemed like this was an issue of interest and importance. The group had a mix of experience and type of work. One of the midwives was newly registered, had a small caseload but was about to give up LMC midwifery and work in the hospital. She had found case-loading as a new practitioner very hard. Another worked in the outer part of the city based in a primary birthing unit and had a full-time caseload. The other two midwives also had full caseloads, and one was about to stop practising as she felt that she was getting 'burnt out' and needed a break. There was, as I had anticipated, lots of interaction between the midwives as they shared their perspectives. Half the group then were about to leave LMC practice, not necessarily permanently, because of the challenges it had posed. 
There were three areas of discussion of particular interest in this group. The first was that a positive interaction with the secondary maternity service relied on having developed a positive relationship with the obstetrician. This became very difficult when they were required to refer to registrars instead of directly to the obstetrician. This was particularly difficult for the new practitioner who found that because she was less well known she had fewer options. In general the discussions about their place in the primary/secondary interface represented one of lesser power. Negotiation, where possible needed to be underhand, and it was the secondary service, in particular the obstetricians, who were in the dominant position. The midwives had to rely on building a relationship before they were treated as knowledgeable professionals in their own right. They sometimes felt insulted by inexperienced and unknown registrars. This coupled with a considerable amount of anxiety about the medico-legal environment meant that this interface could be fraught and difficult. There were different positions taken as to how they managed this, from developing clear, non-negotiable boundaries by one midwife, to the development of 'grey area' by another where the midwife attempted to mediate what she thought the needs of the woman were against the demands of both the secondary service and the medico-legal context.

The discussion about whether they should continue to care for women 'at risk' was particularly interesting. There was initially general agreement that this should occur but they gradually began to qualify this as, one by one, they presented the difficulties that midwives can face in attempting to do so and to rationalise why it would be acceptable not to. They then started to discuss it from the woman's perspective and shifted their position back to how important is was to provide continuity. There was then some dissonance and shifting within this position.

There was also considerable discussion about what they considered as normal. One of the midwives had made a clear distinction between what she considered normal in a holistic sense and what the obstetricians did. She felt she kept these positions separate. Others said that what they considered normal had grown so that it was a notion too hard to describe. This was principally because what the woman considered as normal was at such odds with what they might consider normal. Risk then became intrinsic to the notion of normality so that to separate out 'risk' from the 'normal' 
became meaningless. This also pointed to the centrality of the woman's position in the way that they provided care. Caring for the normal woman meant caring for all. Negotiation and referral was managed according to the woman's position, not according to the midwife's. Laughter made light of some real difficulties.

The midwives were very interested in the results of my survey and I was pleased that I presented them. As hoped, I got several ideas about what further data analysis I might do with the survey and they were pleased to be involved in this process. They were particularly interested as to how 'years of experience' and 'distance from the hospital' might relate to the number of referrals and to the attitudes that the midwives might have to the medico-legal environment and to the primary/secondary interface. Although it was not the aim of the group to achieve consensus this was in general what happened to varying degrees. The midwives built on each other's comments to provide a picture of how they were positioned.

\section{Group Two}

This group was held in another urban area with tertiary level maternity services available. This was a different experience from the first group in many ways. I decided to hold this group in the afternoon before the local College of Midwives meeting to which I had offered to present the descriptive results of the survey. We did not therefore discuss the survey results in the group. This second group was held in the lounge of one of the participant's homes. I was happy to talk with midwives wherever it was comfortable and this home was one in which the midwives quite frequently had their group meetings. Four midwives attended the discussion. The group consisted of three midwives who were all very experienced as independent practitioners. One focused mainly on home births and had also had experience of independent practice in the United Kingdom. Another of these midwives had experience as a midwifery advisor within the medico-legal context. Two of the midwives worked together in the same group practice and had not met the others before. Two of the midwives had a full-time caseload and one was a new practitioner.

This discussion too was lively and stimulating. Again I was struck by stories - lots of stories, illustrating the differing perspectives and reinforcing attitudes. This region was characterised by many choices in the way they could make referrals. There 
seemed to be no fixed process. Again the relationships that the midwives had with the obstetricians were crucial. The midwives could also choose the obstetrician according to his or her perspective or particular skill. They could 'work the system' according to what they thought would be best for the women. They clearly preferred obstetricians 'of like mind'. Lack of consistency among the obstetricians was a problem for them.

All the experienced midwives had a keen awareness of the medico-legal environment. Three had had some experience of it. One provided expert advice, one had had a case taken against her and another had been to court as a witness. They all however had incorporated it in different ways into their practice. This varied from an acute awareness in everyday practice decisions ( the medico-legal advisor), to trying to put it in the background so as not to have 'negative energy' around, (action taken against her) to consciously discussing the risks with all her women and then feeling comfortable with it ( the court witness). All were accepting of the others positions. The new practitioner was finding it all a bit of a mystery!

When I asked them what it was like to discover a complication in women that required a referral they all agreed that it was a difficult situation in which loss and grief was experienced. They felt that loss more keenly for the women who had planned to birth at home or in a small birth unit. They too mentioned the 'grey area' of practice where decision-making was difficult. All the midwives thought that a woman should have a midwife no matter what. One of the midwives, interestingly the one who did mostly home births, really liked the challenge of being able to do some high risk work as well but there was general agreement that there was a place for midwives who didn't. There was an assumption that in these cases the midwife should continue to provide support.

They differed considerably in the way they communicated risk to the women in their care. The midwife who did mostly home births was quite explicit about communicating the range of risks to the women in a frank and comprehensive manner. The midwife who had earlier commented about the 'negative energy' created by being over anxious about the medico-legal environment further commented about negative energy created by heightening anxiety over all the possible things that could go wrong. They mediated this position by agreeing that the different 
personalities of the midwives would mean that risk would need to be communicated in different ways.

\section{Group Three}

This was the first rural group that I visited. I met with the midwives at the local maternity unit where they meet every two weeks to discuss issues of concern and where they have a teleconference with the obstetricians from the secondary care service, which is an hour away by car. As usual I brought along some food but they were already participating in a feast and obviously valuing their time together. The seven midwives who attended had a very broad range of experience. They had been registered from 3 to 35 years and had worked in a variety of areas and countries. In fact it was the most varied of all the groups. Some of the midwives lived in the town where the small rural hospital was located and others were scattered around the area. This group had a cohesiveness that was surprising given the diversity within the group. One of the midwives from this group was about to leave practice due the demands on her time and the increasing needs of her family.

This group were all very positive about their relationships with the obstetricians. They had a fortnightly teleconference and one of the obstetricians came every week to conduct an antenatal clinic. They felt very comfortable about ringing the obstetrician at any time of the day or night and were confident of getting helpful advice and support. There was no choice of obstetrician but this didn't appear to be an issue for them. The only problems they had were with the tertiary service. When they needed to contact this service they were not allowed to speak directly with the obstetrician but had to talk with a registrar who usually didn't know them personally and was unfamiliar with their area. They were in general positive about the referral guidelines. The medico-legal context was not overly worrying although there seemed to be some consensus that they were a bit more defensive than they needed to be. They did not discuss this much at all. They perceived that their own risk was more related to their physical safety from working in isolation or from traffic accidents.

They also viewed risk as emanating from the personal relationships with the women. There was considerable discussion about the nature of trust within the relationship with women. My impression of this group, as it was with the others, was that all the 
issues discussed were reflected on primarily with reference to the needs of the women. Although we were, in general, talking about their practice the conversation returned often to the women and their needs. However this group also discussed the problematic nature of this relationship if the women were not fully honest with them. Several of the midwives spoke about needing to rely on their 'gut feelings' and to be vigilant where they felt there was distorted communication and poor understandings. In this group then there was less concern about managing the physical risk factors or the medico-legal environment, but more attention directed towards the communication within the relationship.

This group also talked about the 'grey area' between primary and secondary care. They were often expected to provide antenatal monitoring for 'high risk' women who were under the care of the secondary service but unable or unwilling to travel the distance to the hospital for monitoring. They were torn between helping the woman in isolated rural areas and the time and effort required to do this for no payment. They shared several experiences about how they had managed this. If they transferred to the secondary care service they were often expected to stay and continue care. If they didn't they were made to feel guilty and that they were letting the woman down. Some of the midwives preferred to make the journey home and others would stay depending on how close the woman was to delivery. They were in general poorly supported by quite a number of the core midwives who would not provide support and even expected them to travel daily to the hospital for postnatal visits. The obstetricians, they said, were more concerned for their welfare than the core midwives were.

The group shared perspectives about what 'normal' meant to them. One of the midwives thought she focused on the normality of the process unless anything became abnormal. Another was vigilant and never really relaxed until the birth was well and truly over. They did though in general agree that there was a wide variation in normal and that the definition has changed over time. I asked then how it felt when risk was identified and referral was required. They spoke of feelings of failure and disappointment not just for the women but also for themselves. They also discussed how it felt in emergency situations that were terrifying and left lasting impressions (at this stage they launched into more storytelling!). 
Our short discussions about why risk was so prominent led to discussions about selfishness and lack of self-responsibility which led to a wee moan about women (especially about the young ones) who expected far too much of midwives. They felt that the demands and expectation of some of the women could make the job impossible. There was a lot more story telling and much laughter. The group did not have time to hear about the results of my survey as they had another meeting to go to but were keen to see the results published.

\section{Group Four}

This was another rural group. They had happily gathered together on my account. This area has a small birthing unit and so all the midwives in the group worked closely together in both supporting each other and in keeping the birthing unit running. We met in the birthing unit. It is a small house with a very homely feeling. There had been a birth overnight and the fire was still on and the house warm as toast. The place had a simple vibrancy and yet felt calm and pesceful. Many of the issues were similar to the previous group although there was less support from the secondary service. There were more wonderful stories, lots more laughter and this time a few tears. They seemed to relish the discussion and were very interested in the survey results.

There were five midwives in this group. Two were very experienced; one had a reduced caseload due to illness. There were two who were into their third year of practice. The fifth midwife had come to the area from an isolated rural practice and was really appreciating having a supportive group to work with. She was only in her second year of practice. The two older, more experienced midwives were clearly an important support for the new midwives. Yet they managed to do this I noticed while remaining very collegial. They did not dominate the discussion. This area is also about an hours drive away from the secondary service and was not considered to have very positive primary/secondary interface. Again, the relationships with the obstetricians were central to how easy the interactions were. Who was 'on duty' made a big difference. As in the previous group the support from the midwives in the secondary hospital was often lacking and was a source of much distress, heightening the level of medico-legal anxiety. The area where the birth centre was based is an area of considerable deprivation and has many families with financial and social 
problems. The group commented that there was little appreciation of this by the obstetricians. As in the previous rural group, the midwives found themselves providing the antenatal monitoring of 'high risk' pregnancies on behalf of the secondary service with little security and no funding.

This group was very woman-centred. The midwives felt they needed to balance the demands of the referral guidelines with what they saw as the best interests of the women. Where women were 'non-compliant' they needed to be more cautious. One of the newly experienced midwives had stopped being responsible for care when women had been transferred as she had 'got into trouble' at one stage. She did however continue to provide support. The other midwives all continued care although they hated doing inductions due to the lack of support at the hospital. They felt more isolated and alone in the hospital than they did at a home birth in a rural environment. The two midwives in their third year of practice felt they were very medico-legally anxious. They felt this was a factor of their last year of training which had concentrated on the 'abnormal'. Also during that year there had been a highly publicised case of a midwife going through the disciplinary process. They though that all their class had begun practice scared.

There was much discussion about whether midwives should care for only the 'normal' and it became clear the definition of normal was problematic. The midwives contradicted themselves and each other during the discussion. Comments varied from not believing that there was such as thing as 'normal', to normal being the absence of medical problems. It was however seen that midwives themselves had to establish the boundaries of their scope of practice yet they also proposed that midwives belong with all women who are pregnant. They discussed the problems of both normalising the abnormal and also the problems associated with 'abnormalising' the normal. There was a general feeling that almost all women were complicated to some degree.

It was clear throughout this discussion that this was a coherent and supportive group who knew each other well and trusted each other. There was a high degree of agreement about the issues discussed. The midwives built the discussion by drawing on each other's comments. They 'used each other' a lot if concerned and were 
unanimous in the benefits of giving birth out of a secondary maternity facility. They even told each other's stories.

\section{Group Five}

I chose to conduct this group in an area where the primary/secondary interface was working particularly well. This is an urban area with a secondary maternity service. Unfortunately there had been some confusion over the venue and only one midwife came to the meeting. Although the group process was absent, the quality and type of data from this interview was such that $I$ have chosen to include it in the analysis. It did provide a perspective that was not found in the other groups. The midwife and I had a lively discussion. She was an experienced midwife and liked to tell lots of stories. She was very well supported by both the obstetricians and by the core midwives. This was the only discussion that occurred actually in the hospital. All the obstetricians in this region she said were supportive and were not interested in taking over the women's care. She called the women 'patients' and said that if in doubt she always refers. She had never had a problem with a referral and has never had a woman complain about a referral. She felt that the other midwives in the area felt the same. She felt that both the midwives and the women were at risk if referral didn't happen. She also contradicted herself as although she said that if in doubt she referred later in the conversation she stated that her philosophy of care was 'if in doubt do nothing'. In this area it was assumed that the midwives would continue care into the secondary context. It was not expected that the midwife would cease contact. This area was the only one I visited where, if LMC was signed over and midwifery care was still continued, then the LMC midwife would be paid by the secondary care service for doing so. She simply needed to send in the bill. However even here the presence of the medico-legal environment was very real. This midwife had had a case taken against her and had 'perfect' support from the two obstetricians involved. Even so it has made her consider whether or not to continue in practice. She did continue because she 'loved it'.

\section{Group Six}

This group was the last and largest group. Nine midwives who are all members of the same midwifery collective came to this group. They were keen to participate and had a variety of perspectives and opinions. This group was held in an urban region. The 
nature of the relationship with the secondary service in the region was regarded as medium to poor although several members in the group spoke of how it had been improving. This group of midwives had their own house in collaboration with their consumers and it was here that we met. There was a variety of experience within the group. There were several new graduates and some very experienced practitioners. Several were involved in midwifery education. I presented my research after this meeting to all the midwives in the area so we did not discuss it in detail as a group.

The nature of this group followed all the others to the extent that the communication between the midwives was developmental; the midwives offered comment to build on those made by others. The thing that distinguished this group from the others was the degree to which they offered contrasting opinions about both their relationship with the secondary care service and with risk and its management. Some found the primary/secondary interface about 'as good as it could get' and others were less happy about how it worked. This was the only group that discussed that the midwife's own confidence might impact on how she was treated. They also spoke about the problems that new practitioners had. One of the participants spoke about being 'eaten alive'. Knowing how to 'work the system' was important. There was also some appreciation of seeing the difficulties that the obstetricians must have in dealing with a large number of different midwives.

The midwives discussed how they communicated risk to the women they cared for. They tried to protect the woman from anxiety and fear. Language was seen as important. They also spoke about the wide variations of perspectives of the women they cared for and how this made a real difference in how they approached discussions. Some were happy to go outside the guidelines if the woman was strong in her position. When a woman wanted to do this, one of the midwives chose to get a second opinion so that the woman would hear another voice. Another chose to keep her away from the hospital to protect her from added pressure. The medico-legal aspects of these positions were discussed. There was general consensus that however much they didn't want to admit it they did practice differently in and out of the hospital system. Their risk management processes were therefore sometimes quite different. Making sense of the different world views of the women was important. 
There was general agreement that midwives should be with all women when they gave birth. One of the midwives who did mainly home births talked about her wish for skilled core midwives who could take over. Another added that they would like to be able to work alongside them. One of the midwives wanted to be able to trust the core midwives enough to be able to go home to bed when they were very tired. Another of the midwives mentioned that women are adults who are responsible for their own lives but others talked abut how vulnerable and scared women can be at these times.

\section{Coding the documents}

Having decided to conduct focus groups it was important to code the transcripts in a way that could capture the nature of the interaction and provide the context; what was going on and what went before were seen as important. The conversations were therefore coded as 'talk' (Myers \& Macnaghten, 1999). Nvivo computer software was used as a tool to facilitate the coding process. There were no predetermined categories used to code the data. Instead attention was focused on the data and the coding categories emerged (Morse, 1994). The question I asked of the data was; 'What is this about and what is being said here?'

Each of the transcripts was systematically coded into nodes. These nodes did not consist of single words, phrases or lines but were sometimes the whole of a person's comments. The segments often crossed over into another's discussion. Most of the data was multiply coded as the 'talk' could be viewed from different perspectives. For example passages which were coded to 'informed consent' were also often coded to 'women centred'. As the coding progressed, thoughts and ideas were recorded in a memo associated with each transcript. A research journal, which covered larger issues, was also kept and added to during the process of coding.

The number of nodes used for each transcript was similar. The number of nodes in each transcript varied from 48-53 nodes, except for the interview with the one midwife, where there were 24 nodes used. Most coded sections had between 5 and 10 lines of text. As the coding progressed the number of nodes increased. It became clear that earlier pieces of transcript might also have segments of text that could be coded to the new nodes. To this end the transcripts were regularly reread and 
recoded. The final task was to listen to all the tapes again paying particular attention to how the talk had been coded. By the end of the coding process all the transcripts had been read and coded using all the nodes as a reference points until saturation had occurred. It became apparent at this stage that some of the nodes were really referring to the same issue. These nodes were merged. The original 53 nodes were therefore reduced to 48 . Some of the codes were about very similar issues and these were groups together into 'trees'. The nodes then hold within them the variety of viewpoints expressed by the midwives, both negative and positive. They also hold the midwives' interactions.

\section{Creating themes}

The challenge from this point on was to take some meaning from this talk. What was it about this talk that might illustrate how midwives make sense of what they do at this point of practice? It was clear that further analysis was required. The coded data needed to be related and grouped. Although NVivo software does have a facility to assist in doing this I found it limiting. Instead I made large coloured cards of each node, spread them over the floor and walked around clustering and reclustering according to whether they had some relationship or flow-on effect. In a sense it was the time to play. This happened over period of some weeks and indeed over months as the grouped codes were eventually transferred onto the wall. The data were therefore grouped and regrouped. There were some codes that fell together naturally and others that were more of a moveable feast; there were many places where they could have belonged. The text within the codes was frequently reread to ensure that I had a sense of what they contained. Once I had developed what was beginning to be a pattern I revisited the survey data to explore how this might speak to the developing themes. This assisted in the final placement of the data. This was a process of synthesising, where categories were connected up, decontextualised and sifted (Morse, 1994). This process of data analysis is similar to that of grounded theory but could more appropriately be called a conceptual description (Stern, 1994).

What I was doing was to construct themes, areas where connections were established. Eventually I came to a place where further movement added little to the understanding. At that stage the codes had been gathered into four themes. I called these themes 'being a professional', 'working with complexity', 'working the system' 
and 'being with women' (Table 7.4). These were not necessarily mutually exclusive; some of the codes could have been included in other themes. However the decision over final placing related to where I thought they were the best fit.

In a sense this representation appears particular linear and over simplifies the complexity and interrelatedness of the 'talk'. The themes and sub themes are in actual fact not separate but merge and speak to each other. For example, the codes 'protecting the mother', 'being in the grey area' and 'handing over care' can be seen as very interrelated; in attempting to protect the mother both from anxiety and from unnecessary intervention the midwife exists in the 'grey area' where the decision to seek referral is possibly necessary but not yet made. She is, in a sense, resisting handing over care. The connectedness of all the themes becomes apparent as excerpts of the dialogue, provided in the following chapter are read. There are flavours of other themes held through all the excerpts. 
Table 7.4 Themes and coding categories

\begin{tabular}{|c|c|}
\hline $\begin{array}{l}\text { Being with women } \\
\text { Holistic care } \\
\text { Women-centred } \\
\text { Staying LMC } \\
\text { Being at risk } \\
\text { Having a relationship with women } \\
\text { Trust } \\
\text { Protecting the mother } \\
\text { Informed choice } \\
\text { Options } \\
\text { Communicating risk } \\
\text { Minimising risk }\end{array}$ & $\begin{array}{l}\text { Working with complexity } \\
\text { Being normal } \\
\text { Variation in practice } \\
\text { Paradox in practice } \\
\text { Flexibility } \\
\text { Grey areas } \\
\text { Doubts and mistakes } \\
\text { Decision-making } \\
\text { Gut feelings } \\
\text { Safety } \\
\text { Multiple world views } \\
\text { Disciplinary boundaries } \\
\text { Differing perspectives } \\
\text { Variation in practice }\end{array}$ \\
\hline $\begin{array}{l}\text { Being a professional } \\
\text { Being skilled } \\
\text { The awareness of risk } \\
\text { High risk care is stimulating } \\
\text { High risk care is difficult } \\
\text { Experience } \\
\text { Confidence } \\
\text { Income } \\
\text { Midwives choice } \\
\text { Identifying risk } \\
\text { Home vs. hospital } \\
\text { Rural practice issues } \\
\text { Being accountable } \\
\text { The medico legal environment } \\
\text { Taking risks } \\
\text { Blame } \\
\text { Safety } \\
\text { Stress }\end{array}$ & $\begin{array}{l}\text { Working the system } \\
\text { Managing relationships } \\
\text { Relationships with obstetricians } \\
\text { Communicating care } \\
\text { Communicating with each other } \\
\text { Core midwives } \\
\text { Private vs. public } \\
\text { Using the referral guidelines } \\
\text { Risk identification } \\
\text { Second opinion } \\
\text { Handing over care } \\
\text { Working outside guidelines } \\
\text { Power and authoritative knowledge } \\
\text { Collaboration } \\
\text { Registrars } \\
\text { Control } \\
\text { Who defines risk }\end{array}$ \\
\hline
\end{tabular}

\section{Trustworthiness - goodness and truth}

Can we trust these results and can we use them? Are they valid? The issue that needs to be addressed regarding this interpretive, intensive part of the research are its internal or face validity, and its generalisability or external validity.

Both Reed and Roskell (1997) and Krueger (1994) state that focus groups have high face validity due to the credibility of participant comments. Krueger comments that face validity

$\ldots$ is the degree to which the procedure really measures what it proposes

to measure...Focus groups are valid if they are used carefully for a 
problem that is suitable for focus group enquiry....Typically, focus groups have high face validity, which is due in large part to the believability of comments from participants. People open up in focus groups and share insights that may not be available from individual interview, questionnaires or other data sources ( pp.31, 32).

Krueger does however recommend that users of research have some faith in the procedures but also retain a healthy scepticism in research results however they are obtained. In this study the choice of focus groups is appropriate to the question posed. In Chapter 3 I have established how risk was operationalised and why focus groups were chosen. In this chapter the rationale for the choice of participants and the detail of the process and analysis of the research are explicit (Brink, 1991). Participants' comments are included in the following chapter, as the model is developed.

The issue of the generalisability or external validity of this intensive aspect of the research is more problematic. Despite the more relativist approach, that research results gained this way cannot be applied in any other setting, it is natural for the reader of research make assumptions of this sort. The critical realist approach claims that all research should have generalizing claims and that in order for the research to be useful some degree of inference is essential. It distinguishes between two different types of generalisability. Empirical generalisability occurs where statistical extrapolations can be made to larger populations and that there are established mathematical approaches of assessing the validity of these generalisations. The other type of generalisability, it proposes, relates to the deeper domain of reality where the generative powers are uncovered. The difference is in the type of inference made. Bhaskar's abduction and retroduction occur where the researcher abstracts creatively from the data. The problem with these types of inference is that there are no fixed criteria for assessing them.

Waterton and Wynne (1993) also comment about the difficulty of assessing interpretive research. They conducted focus groups in their study of community attitudes associated with living close to a nuclear power site and commented that validation could only come from: 
...a kind of open-ended peer review in which our conclusions have to stand up to scrutiny by other researchers, by the community under study, and by others who read the research results. This process is less to do with 'quality control' than it is to do with trust in the research (p 65).

Williams (2002) makes a much stronger case for generalisability within interpretive research provided the generalisations are made cautiously and within the context of pluralistic research. He points out that the relativist approach, which rejects any notion of applicability in wider contexts, may be useful in a contemplative sense but that increasingly interpretive research is being required to inform policy and practice. It needs to be able to assist in the understanding of social structures. In order to do this he proposes a type of generalisability he has called moderatum generalisation where aspects of situation can be seen to be instances of a broader recognisable set of features. His position, that the cultural consistency (not cultural homogeneity) of the social world means that "...the existence of shared norms, a common language and physical referents can allow at least some reciprocity of perspectives between researched and researcher, as well as viable comparisons between the validity of statements" (Williams, 2002, p137). He stresses that knowledge derived through interpretation is fallible and incomplete and favours the use of methodological pluralism, in effect, a critical realist approach.

Sim (1998) also proposes two different categories of generalisation. She commented that focus groups do not provide representativeness in the same way that a survey might. She proposed that interpretive research generates theoretical generalisations and more hypotheses than conclusions.

There are several approaches I can take to the generalisability of the research. I could take a relativist approach, reject any attempt at generalisability, and offer the interpretations as a personal expression, as a gift for individual midwives to ponder and reflect on. In essence this is a contemplative approach. It does have the potential to do this. However I do think it can be useful to practitioners, educators, and policy makers. To this end I claim a critical realist approach. Given that it is conducted and will be interpreted alongside an extensive research method and that it is explicit in 
both positioning and process, it does have moderatum generalisability. It is a position that does not claim truth in any fixed state. It recommends caution and invites debate.

\section{Conclusion}

Focus groups have been used as the intensive research method to provide insight into how midwives make sense of risk in practice. This chapter has provided the detail of the process and the initial, or first level analysis of the data. The groups provided valuable insight into the midwives' perspectives. I felt enormously privileged to have been able to share the midwives' stories. Our discussions were vibrant and heart felt. We had laughter, tears, good food, and coffee and sometimes wine. The midwives were without exception generous with their reflections and engaged and thoughtful in their responses. I was personally both encouraged and healed. They spoke eloquently and passionately about their work and the women they cared for. The following chapter provides further interpretation of the data and incorporates the extensive research findings and associated theory. It introduces and explains the theory developed from the research. 


\section{Chapter 8: Thematic Analysis and Interpretation}

\section{Introduction}

This research seeks to understand how midwives make sense of risk in practice both in what they do and in how they think. In doing this it uses the philosophical approach of critical realism, which addresses the complex and multi-layered nature of reality and thus the importance of investigating such issues through multiple lenses. The representation of the findings of such research poses considerable challenges, as investigations that seek to make sense of complex issues resist a simple linear presentation. The results of the survey have been described in Chapter 5 and a first level interpretation of these results has been presented in the survey discussion in Chapter 6. This chapter moves the research to the next level of analysis. It presents the findings of the intensive part of the research, the focus group discussions, and incorporates the survey findings within constructed themes. In this way the focus group data can provide context and depth for the survey data, and the survey data can provide support for the themes extrapolated from the focus groups.

Throughout this chapter there is considerable inclusion of segments of the participants discussions. They are used as illustrations of the themes and sub themes and provide a sense of the focus group process. Sometimes only one participant is included in an excerpt. Often a whole section of the discussion is included, providing a clearer indication of the contextual nature of the groups. Focus group data however are very 'messy' and much of the data were multiply coded. I have endeavoured to find a best fit, the place where they were most appropriately placed. Some of the midwives were more articulate in their responses than others, so small modifications to the wording have occasionally been made in order to make extracts readable. This has consisted of such things as deleting some of the verbal mannerisms or repeated words. Where inaccurate grammar reflects hesitation, it has been left unchanged. Non-verbal responses, such as laughter or murmured agreement $(\mathrm{Mm})$ are included where it relates to the midwives reactions. In order to aid in the understanding of some excerpts I have needed to add words. Where I have done this my words are bracketed, [ ]. Where less intelligible sections have been omitted I have placed three 
dots .... Each segment of data begins by identifying the group from which it came. Each participant is identified by 'F', followed by a number allocated to her. Where my question is included, ' $Q$ ' precedes it. In order to protect the non-identifiably of both the groups and the participants I have removed names of regions, hospitals and people, with brackets [ ]. I have also done this with one of the physical conditions described as this is a rare condition and is localised within one family grouping thus making that region identifiable.

\section{The themes}

The themes presented throughout this chapter are interpreted in relation to risk in general and also to the risk theory presented in Chapter 3. This is done in order to both reflect the midwives' experiences of risk and to provide some empirical evidence to either support or challenge the assumptions about risk and its manifestations made in the variety of risk theory presented. The themes developed and the commentary on risk theory lead to the development of a model for midwifery, which is presented in the following chapter.

\section{Being with women}

The central theme that has emerged within this research is that of 'being with women'. This was quite clearly important for the midwives both in what they did and in how they felt. The survey revealed that the lack of a formal screening process at booking and the degree to which midwives continued care into the secondary maternity service, sometimes even when they did not receive payment, are strong indications of their commitment to being with women despite the level of risk. They overwhelmingly disagreed with the statement that independent midwives should care only for low risk women. The focus group data confirm and support this. Throughout the conversations the centrality of the position of the woman was revealed. The midwives went to some effort in order to support and be alongside the woman on her journey into motherhood whether or not she required obstetric assistance. The discussions were heavily laden with stories from practice, connecting the midwives with the women for whom they cared. The real, complex and challenging work of meeting the needs of the women was paramount. 


\section{Holistic care - 'being their anchor'}

Rather than the presence of risk being seen as an impediment to participation, the midwives often saw that their input was even more important when increased risk had been identified. They had a holistic view of the woman rather that compartmentalising her into a low risk or high risk 'case'. The following extracts illustrate that the midwives saw the importance of the social and emotional aspects of midwifery care and that these needed to continue even when risk was identified:

\section{Group 2}

F4

I find it interesting, even just the subject of what it's like being handed over to hospital care and not having the midwife with you. My suspicion is that for the woman to have the midwives with them, there's still aspects of their care that are normal for them and so those things in a crisis situation can get lost. That there's someone there who, even though decisions have to be made really quickly, that person is still capable of what they can decide, making those decisions on how it's going. When you think 'gosh you know we need to do this right now for the best outcome but how we can do this?' And I think if the midwife's there, then for the woman there's more of a 'well how can we do this that it's best for the woman?' Because we know it's got to be done and we know that we can't continue as we wanted to, as she wanted to. But maybe she can still feel like that she's a part of this and not 'done to'

F1

And I think that, she's having a baby. You know the family and you can keep holding onto that rather than get lost in all the technology.

F2

Yeah she's a woman having a baby who's got extra risks or conditions. She's not a case who happens to be pregnant.

F3

And you're not only dealing with the clinical side. You're dealing with the woman's emotional expectations and processes and the family's. Because you're their anchor. You're the one they have known all the time.

\section{Group 3}

F5

It's not just one thing with them. It's a combination of things, and it's holistic

F3

You're trying to focus on wellness. Like, a lifestyle thing. You try to encourage people to take care of themselves and take some responsibility for themselves.

F2

Also it's also education and that sort of thing. To me that's caring. 
F5

Yeah I spend a very long time in each visit - talking, talking, talking so in the end they're confident.

F4

I guess the bit is about what we see midwifery care is too. Because I know for some of my colleagues midwifery is care of this pregnancy. Whereas for me midwifery is caring for this woman who's pregnant and so it is caring for her social side. And being aware of and being involved with the woman and who she is, her social situation and her relationships with her husband, mother and whoever else. And what's happening with the children and money and those sorts of things. Because to me they impact on the woman and what's happening for her.

The midwives' work then seems to be both defined by risk and yet not defined by it. They are there because risk exists. Yet there is more to their work than this. The midwives often talked about the normality of childbearing in a sense that was different from the purely physical and used this to justify the reasons why they should stay involved

\section{Group 1 \\ F1}

Who will look after the women, the at risk women if they don't have midwifery care? I mean it's the only thing that may be vaguely keeps some sense of normality along the way.

\section{Having a relationship - 'continuity and trust'}

For many of the midwives trust was a central part of the relationship that they established with the women over the time they got to know them. This relationship was seen as even more important when risks were identified

\section{Group 2}

F2

I would also say that in high-risk women often the risk is minimised by that continuity and trust and they are so much more relaxed. So you actually can often alleviate some of the symptoms and side effects.

\section{Group 6}

F3

Yeah, and it can be that emergency response or it can be that anticipation of guiding them in a different direction and helping them to make those decisions. And they trust you to do that because we've worked with them through their pregnancy. Worked up to that point. 
The midwives valued the relationships that they developed with the women. They did see however that the risk environment threatened the development of the trusting relationship and distanced them from the woman.

\section{Group 2}

F2

And I was saying at a meeting the other day, my midwifery's changed from the early nineties to now. I see myself as in a much more risk situation in that relationship with the client than I did when I first started going to home births, where that relationship was really close and trusting and very friendly and warm. Almost like a warm fuzzy sort of relationship. And now I feel like I'm removed because I'm protecting myself really with more documentation, more knowledge of what can go wrong and what has gone wrong for other midwives and just more awareness of how I've got to protect myself in case there's an adverse outcome because of the decisions I make. So, yeah, to me it's about seeing what's happened over the last ten years, seeing what's happened through ACC and the Health and Disability Commissioner's work and perhaps being aware that women want perfection from us. They're shopping around. They're not happy to have an adverse outcome or even an outcome that is less than great. I'm talking generally because I know a lot of women are very reasonable. But there are more women who are wanting the perfect birth. And they're expecting us to provide it.

Within practice, apart from being effected by the risk environment midwives also had to establish relationships knowing that partnership, where it existed, was tentative and where trust did not exist, risk was increased.

\section{Group 3}

F3

But you can get, patients being patients. They can say one thing to you and if there's a problem later on they deny you've said anything.

F2

Yeah

F1

You have to write it down

F3

I know. But like this case that I've just referred. When I offered her amniocentesis, both she and her partner very emphatically said no. And they didn't want to see an obstetrician either and then about two months later they said 'no you didn't say that'. I said 'I've written it down and I've told my other two partners about it as well'. Mind you I probably said something over here about it too. But they both denied it. Q

It's sort of illustrates how closely the risk we manage in the women that we look after links to our own risk. 
F3

Well there's a personal risk to me and I did make a note of that particular one. Because he's just come out of prison after murdering someone and while he was on leave he raped another woman. So I have to be pretty careful.

Q

How do you manage risk in your own practice? When you're caring for someone, what are the main things you do to keep yourself safe in practice?

F4

Listen to your gut.

F1

Yes, yes. Instinct.

F3

Well in this particular case I've advised my partners.

F6

And consult.

F3

And consult, yeah. Yeah.

F1

Make it clear that the women and their family, understand what's going on. Just if to say something develops that everything was normal, going along normally, then suddenly something developed that's not going to be normal and that's where the communication can be distorted.

F2

I think [the lawyer's] guidelines are quite good too for people to avoid, and, you know, keeping safe.

F2

There's some quite distinct characteristics of cases that are probably the best ones to keep away from, you know. As far as personal safety, too. I know a lady, had a chat to her husband to say 'is it okay you're going there?'

F4

Mm, like when I say listen to your gut feelings. That sometimes it's actually the most reliable form of information. Just in the sense that somebody's not being entirely honest with you or you just know that something's not quite right and so you are perhaps a little bit more vigilant in what and how you write things down. And in some cases I've taken photocopies because my women carry their notes. I actually will even take a third copy so that I still retain the notes in case any of the notes go missing.

F3

$\mathrm{Mm}$

F2

$\mathrm{Mm}$. 


\section{Informing choice -'things can change'}

The way the midwives in the study provided information and support for the choices that women made in relation to risk reflected a more value-laden approach than an epidemiological one. It seemed that the connection that the midwives had made with the women and their families facilitated decision making that was more related to how the women perceived the problem rather than primarily to the scientific evidence. The following excerpt, related to the decision about whether to give Vitamin $\mathrm{K}$ to the baby at birth is typical of the way the midwives spoke

\section{Group 6}

F2

I often ask them about their decision making processes and how they make decisions in their ordinary life about things and how they might make decisions as parents and that this actually may be one of the first ones that they will be making. And whether they like to make decisions that are based on research or decisions that are based on their life philosophy or decisions that are based on doing something to protect their baby, or doing something that is perceived to be seen as protecting your baby, or not doing something that is seen to be protecting their baby. And I so often ask them to sort that out first and then when they come back and they say 'oh well this is, this is our world view'. Then I know which way to approach it with them. Which means it isn't just a sentence slotted into a tick list.

F1

You go with Vitamin K?

F2

And some people will say 'Well we gave it to our other three we'll give it to this one'. And you go, 'fine!' Yes because that's their decisionmaking process. And others will go, 'we gave it last time but we didn't understand what we were doing and we want more information'.

F3

And of course there are some that will say 'oh I don't care, you know, do what you think'. Most people do. With most people it's quite a common. F4

'What would you do or what have you done?' That's what I get often.

F2

Yeah, true.

F3

It's very hard though, that whole thing of informed consent. I mean Vitamin K. There's so much information out there and you can sit there and read most of it and you still come away thinking 'it's such a grey area'. I think it's a really hard issue to talk about and some people have actually given them research articles that they have been interested in and others just want their little wee orange pamphlets and others, will just follow the recommendation from the Paediatric society. 
F2

I usually introduce it by asking have they heard of Vitamin $\mathrm{K}$, what do they know about it? And if they've got a simple answer and they don't want to know any more, they're happy with where they are, then that's fine. But other people do want to know more and you can point them towards the internet sites.

No midwives in the groups mentioned that they provided a statistical indication of risk as a matter of course. They seemed more concerned about reducing anxiety and fear in the mothers and tended to avoid using the word 'risk' at all. They often seemed protective of the women yet at the same time viewed the woman's choice rather than the midwives' choice as both pivotal and final. There was some tension therefore between protecting the mother and informing choice and there was clearly an understanding that risk decision-making on the part of the women was not necessarily a rational process.

\section{Group 6}

F2

I tell them the risk is high if happens to you, it's very low if it doesn't. You'll never know whether you're a casualty or not. (laughter)

F1

That's right yes, it's only $1 \%$, but if you happen to be that $1 \%$ it's too high.

F4

It's difficult though because if you dwell too much on that and they're an anxious person it's going to be very counterproductive. That's the problem. Sometimes too, like, in say a primip wanting a home birth. They'll say things like 'so what would make you have to go to hospital?' And that's sort of like an opportunity to talk about the kind of things they want to experience.

F3

But I don't always think that we actually use the word risk.

F2

No

F1

$\mathrm{Mm}$

F3

I always find myself using words that take it away from increasing fear into saying 'this could change from what you wanted it to be'

F1

Things change

F3

From what you're wanting it to be, you know. So that it isn't seen as something big and scary. But we're also not playing it down as if it's not 
a reality-. 'This will never happen'. But instead of making it something really scary, to anticipate as something bad that can happen. I think the kind of language I use certainly moderates that and I don't talk about risk, you know. 'You're at risk for this, this or this'.

F1

I think if it's the woman's decision and you've gone over it with them, and feel 'yeah!' and it's very similar - if they're wanting to go outside what is normal I'm comfortable with that as well.

F2

You know I mean because there are a lot of things. Like the polycose [testing for diabetes]. I mean, heck! You know very well that most of the time for low risk people, there's no need for that sort of thing. But if a woman wants it, fine, you know? But if they choose not to have it. I mean basically it's got to be their decision and as long as you're happy to stand with them I think it's good

F3

It's not just their decision. It's that wider community as well.

Many of the midwives in the groups were prepared to support a woman's decision when it went against medical advice, provided they felt that the risk was small. The following extract describes the decision-making process that occurred when one of the midwives was caring for a woman with a rare medical condition which had a very small possibility of complication. She had previous delivered normally in the rural birth centre and had resisted the antenatal advice of the obstetrician to deliver this baby in the secondary hospital.

\section{Group 4}

F1

No way I felt she was going to go to [secondary hospital]. She was in her bed and she wasn't in the mood to move for anybody. And I said to her, 'You don't plan on going up do you?' And she said 'No'. And I said 'Okay I'll just ring the specialist, let him know. And that's to cover me'. She said 'Fine'. So I rang the specialist and I said 'I have a situation down here. I've got a lady whose got [complex condition]. She was supposed to be going to [secondary hospital] but now she's in labour she's not going. She's decided she doesn't want to go'. 'Oh rather you than me' he said. And I said 'I'll let you know'... I'm really letting him know so that if I have to transfer in a hurry he knows what the background is.

Q

Okay so you use a referral in that way as a way of medico-legal cover?

F2

Yes

F1

Yeah. But when we started doing that and we used to ring up and say 'Look I'm just letting you know that this and this is happening. So that if we turn up you have an idea'. And we actually got into trouble. We got 
told not to do that; that they didn't want to know. That if we did that we were just, they didn't want any of these 'we're just letting you know'. We're setting them up for problems. They feel they then have to do something about it, so they don't want to know basically.

In their dealings with women the midwives seemed to take on the role of mediating risk for the women. They were, in a sense, attempting to protect the women from anxieties associated with risk, avoiding both the use of the word and thus not presenting the epidemiological evidence in numeric terms. In this way they dealt with the problems associated with the ecological fallacy. That women should approach childbirth with confidence rather than fear was paramount. It was important that they developed a trusting relationship and that the woman had confidence in the midwife's decisions. In protecting the woman from anxiety they therefore tended to take on the risk themselves.

What is uncovered in this research is that 'being with women' is a central part of their practice. The relationships that the midwives develop in the process support them to stay 'with women' as they become mothers. Being 'with women' is clearly then not mere rhetoric but is acted out in practice. The following themes provide evidence of the challenges that midwives face in 'being with women' and in dealing with the risk associated with doing this.

\section{Being a professional}

The midwives in the focus groups saw their skills as being extensive, and that these skills were appropriate to the needs of women experiencing complex pregnancies. It was these skills that they used in the assessment and management of risk. The survey has revealed that risk management is regarded as a very important part of this practice. The focus group data revealed that being a professional related not only to skill development but was also related to being accountable for the use or misuse of these skills. It is within this theme that the midwives 'double-edged sword' of risk is revealed; risk to the mother and baby, and risk to midwives themselves. 


\section{Being skilled - 'that's why you're a midwife'}

The midwives felt that in order to be able to assess and manage the risks associated with childbearing they needed to attain and retain the skills required. For the midwives being a skilled practitioner was important.

\section{Group 6 \\ F1}

I mean that's why you're a midwife. Or that's why people perceive you as a midwife. Because if they perceived it was going to be normal they'd have a friend and you're not a friend. You're the midwife. (general agreement) I mean I think that's part of it. That's what you are. You're a midwife.

F2

Where a woman is having a baby there should be a midwife and her skills should be appropriate to where she is, you know in the big [hospital], in the small [maternity unit]. They should be appropriate to that. It shouldn't be a technician and it shouldn't be a nurse

Being involved with high-risk care was seen by some as a way of enhancing skill development and was related to caseload, philosophical approach and interest. The midwives were confident that their skills were appropriate to the provision of midwifery care for women with complex pregnancies although there was some acceptance that there should be flexibility about whether all midwives should be available to continue care into the secondary context.

\section{Group 2}

Q

So that the whole thought of saying, you know you've become a bit high risk, and I will hand you over to the team. You know, we'll see you postnatally. Do you think midwives should do that?

F1

No I haven't, I haven't ever

F2

I don't think 'should', that word should be used. I think it can be individual, because you do have midwives who want to concentrate on home birth and total normality. And I don't think they should be denigrated or penalised for not continuing in a high-risk situation.

F1

I think as well there's financial considerations if you hand over. I mean I only do three or four ladies a month. If I hand somebody over I've lost about $\$ 1,000$. I can't afford to do that. But also I find it's much more interesting if I've got high risk ladies and I'd much rather do that. I think just doing straight normal births all the time would be very boring. I like having some high-risk women. It makes the job more interesting and I 
love a normal birth as well and I've probably gone too far the other way. I've got a name for myself now as being the person to go to, as an experienced midwife, if you've got any medical problems and can't afford an obstetrician and don't want to go to the hospital, then go to [midwife]. And I've had ladies who really probably would have been better off at the hospital, who've come with me where we have had to go to the hospital quite a few times. But I've really enjoyed looking after them. I feel I'm more useful sometimes.

\section{Group 5}

F1

The thing that gives me the biggest thrill is a previous caesarean section. If I can have her relaxed before she comes in with the thought that she can do it, like she hasn't got disproportion and as long as the labour flows along [she will have a normal birth].

The expectation to stay involved, to manage risk, seems to be a dominant part of independent midwifery in New Zealand. This is supported by the survey, which has shown that $74 \%$ of women who had responsibility for their care transferred continued to receive care from their midwife, and that in $26 \%$ of these transfers midwives who continued care provided at least some of this care for no payment. They continued this care both because of the centrality of the connection or relationships that they had formed with the woman and because they found it satisfying and rewarding. The added dimension of the relationship that the midwives formed with the women meant that they continued care also for emotional reasons. Some of the midwives spoke of the intensity of their own emotional reactions when they identified risk.

\section{Group 2}

F3

It's a horrible feeling really. It's almost a physical sensation of disappointment and anxiety. [You can feel the] loss in your tummy, (other 2 agree). You know we're going to go to a different place. Yeah that gut feeling that's almost a tachycardia as well.

F1

Something has changed.

F3

You say to the woman 'now we're going to have to go down a different path'. And there is a loss of that possible ideal birth that you were looking forward to.

F1

Yeah! That's the word to me - loss. On one level you're dealing with a grief situation.

F2

$\mathrm{Mm}$. 
F1

Because you've lost the normal process that you were hoping for and that was expected. And it is certainly for me a common scenario when suddenly GPH [also called pre-eclampsia] rears it's ugly head.

F2

Or the breech.

F2

$\mathrm{Mm}$.

F2

Yeah and they were somebody who was really down to earth, trying hopefully to have a normal birth at the local unit or at home. And it's taken out of your hands with the GPH and things just start to escalate. And you're not only dealing with the clinical side you're dealing with the woman's emotional expectations and processes and the family's. Because you're their anchor, you're the one they have known all the time. But I mean in the hospital situation it would be different again because you're acting a lot quicker than in an antenatal situation and if we've got something happening here we've got to go there.

F1

I think there's an acceptance when it's in the labour/birth situation. The reason why they've chosen to deliver at a hospital is because they had that need in their personality to feel safe because the doctors are there. That's the only reason why women I care for choose to deliver in hospital. So I don't feel that sense because there's a lot of that acceptance from the woman. I know that that's why she's chosen that type of birth. Whereas with the birthing centre or home birth I have an incredible sense of grief when I have to refer. It's almost as though I know that normal road so well that's the one I like to walk down. And I know that I might limp down it still. But I know that I'm not going to be on my own. It's not that I'm like a mother lioness over cubs or something, trying to defend them from other people. But I know that there's going to be input apart from my own and I can't always trust that. I think that's where my grief comes from. That I have some very unreliable obstetricians who will fill their heads up with all sorts of rubbish. And I have other ones who are so good, who support [the woman] and still try to stay on the normal track. There's this huge gap between the two and I don't feel comfortable because I can't guarantee that we're going to get this one or that one. It could be that one or it might be she's being counselled by this one and seen three or four times in clinic by this one and then on the actual day it turned out to be that one and I think 'oh shit'. It's just about loss of control.

To varying degrees when midwives identified the need for referral they experienced loss and grief. They understood that everything was going to change, that they were now not the only people going to be involved in the care and that others might not be so willing to meet her needs. There was the possibility of loss of control and some increased anxiety about the accountability that might eventuate from an adverse 
outcome. There seemed to be an increased need to protect the mother especially when she had been planning to give birth out of a hospital.

There was also a general feeling in the focus groups that the secondary service expects the LMC midwives to continue care into the secondary service. It was perceived by the midwives that this was related to staffing issues within the hospitals rather than to the institution's explicit commitment to continuity of carer. It may also be that hospitals have reduced the number of core midwives on duty as a response to the high number of independent midwives who continue care when risks are identified and interventions take place. There are also funding considerations. Midwives who do not continue care can lose some of their income and hospitals that encourage the independent LMC midwives to continue care can save money, as they need less 'core midwives' on each shift.

Whatever the reason, continuity of midwifery care from the primary into the secondary context is a defining feature of New Zealand midwifery. For most of the midwives the extra challenges that this posed were viewed positively. It could be a place where they achieved real satisfaction. Risk then could be seen as making midwifery more interesting and more challenging and was related to more fully meeting the needs of women. Many of the midwives in the focus groups expressed this.

For some midwives, especially those in rural areas, it could be problematic. There was some guilt expressed if they were unable or unwilling to continue care once the women had been transferred out of the district and into the secondary hospital

\section{Group 3}

Q

And when you transfer someone from here, do you like to stay with them through to the birth or is it time to come home?

F4

Well we're expected to stay.

F3

We're expected to stay on and that is difficult

F4

Yep and sometimes the staff in [secondary hospital] are a little bit reluctant. Like sometimes you're told fairly overtly in front of the patient, well you're really letting Mary down by going, even though she's quite 
clearly secondary care and so, you're made to feel that you are letting the woman down and sometimes you feel quite pushed into staying.

F5

Pushed into staying there and you feel guilty that, you know, you're made to feel really guilty and that sometimes...that it's really difficult. But they have got, you know there are staff constraints over there too. So you know the pressure just goes along the chain really.

Q

And do you prefer to come home or do you prefer to stay?

F3

Well I prefer to come home because its so tiring. The trip there and then staying and then the trip home. So I just write in my notes 'transferred over to secondary care' and I give it to them and I write it down

F1

I don't think we should be staying. If it's a forceps birth because that comes under secondary care doesn't it?

F2

Oh I stay for a forceps

F5

Not always, not forceps or ventouse. They're just assisted.

F4

But things, like postnatally, it is more of an issue there. Somebody for example has had twins at 34 weeks and the babies are in special care. Then the staff will be ringing us now 'when are you coming over to do your postnatal visit' and would expect us to drive an hour, do a postnatal check and then drive back again just to have a chat. And the pressure comes postnatally which, 'oh but she is your client'. And I would say that obstetricians are usually a lot more understanding and will often either offer you a bed to stay the night. But other midwives will just hang you out to dry.

F2

Horizontal violence with the midwives is just horrendous.

F2

Sometimes you feel like you're wanting to cry and you're just so unsupported.

F3

Yeah

F2

That's right. It's not all of the staff. But there's a huge element and that's the hard part I think.

In both the rural groups I visited there was some distress expressed about the 'core' midwives unwillingness to support rural midwives who had transferred women to the secondary hospital.

Rural midwives also felt that they needed to be more skilled than other midwives, due to their isolation 


\section{Group 3}

F5

A lot of the secondary stuff is up to us and we just have to keep upskilled. We have to go over and learn the neonatal resuscitation and the transfer things [when transferring to the tertiary hospital]. I did one of these recently, and how to deliver a breech. I went to those courses. Because if something's happening we are obstetricians. You know we do want to get to [secondary hospital] in time, but sometimes you can't. So we have to deal with that.

A referral rate of $35 \%$ would indicate that midwives do not make too few referrals. Ninety four percent of the midwives surveyed also thought that their referrals were appropriate to the needs of the women. The fact that the referral rate was stable across regions, practice patterns and attitudes indicate that the level of skill that midwives use in assessing and managing risk is not of concern. Although they were disappointed in having to refer there is no indication in the survey that this prevented them from doing so.

There was one area however where the midwives were less happy with the way they needed to refer. This was related to referrals for prolonged pregnancy and lack of progress in labour and was reflected in the survey by the high number of referrals for these reasons. For the midwives in the focus groups these referrals were the most frequently mentioned areas of difficulty. In the following extracts the midwives acknowledge that taking too long on its own is not problematic provided there is a thorough assessment. They will support the woman's decision to avoid intervention but only if the woman is prepared to 'take that risk'.

\section{Group 6}

F2

Yeah but it is, it is like someone going past 42 weeks. I mean if you are happy like for instance even though you know, you know that the doctors would say 'no that she should have been induced at 41, three or ten days over' or whatever, as long as she's really committed that that's what she wants and as long as everything is okay with the baby you know, all those checks are done and everything like that, I'm quite happy as well but if she's not prepared to sort of stand there and take that risk, well then I'm not either because I'm not going to put my practice on the line.

Q

So you feel comfortable that they understand the risks and that they will take then and not...

F2 
Yeah I had someone just recently who went up to 43 weeks and she was told the risks by me and by about three different doctors and she still stood there. And I said 'oh okay well fine' you know. Because she'd had three antenatal day unit appointments and the baby was absolutely fine so we...

F1

[Midwife]'s saying that she'd get other people to talk with the woman. That's probably one of the most useful referral moments is in actual fact that if you haven't been convinced by the woman when she says 'yes I understand' that if she talks with someone else then she can never actually say to you 'I thought I understood but you were the only one who ever explained it to me so you must have not covered the points I really needed to hear'. I really like it if someone, and particularly if someone who may do it from a different perspective, if she can still stand there and go 'yes, I understand', then yeah!

F3

I actually take the opposite approach. I think of the woman I had that went to 43 weeks. I kept her out of the hospital system. I wanted to protect her and we had talked about the risks of post dates after 42 weeks and I'd actually outlined the risks in her notes and she signed them with me. That as far as checking liquor volume and things we did them through [a private clinic] because I didn't want her to come head to head with an obstetrician or a registrar who would influence her and make her feel unsure of herself. So I probably tend to do the opposite. That was a homebirth and I don't know why I do it but I know I practice differently with women planning to be in hospital or at home birth. I guess it's a different scope of practice.

F2

Its because the women come with different attitudes

F3

It is, it is, it is

Similar concerns are also expressed when women 'take too long' in labour. The following two extracts reveal that the midwives' commitment to avoiding intervention requires that they take risk on themselves either overtly or covertly. There is the underlying implication that the obstetric view is dominant and that midwives attempt to avoid a confrontation with this dominant paradigm.

\section{Group 1}

F2

And what I find I do I set myself little goals like if somebody is maybe in prolonged labour. Okay I'll give them another hour and then I'll do a complete reassessment and we'll see what's happening. I set margins and sometimes I get closer and closer as you get on. But I think it takes quite a toll. Because I think for me, and I would consider myself an experienced practitioner, I'm very conscious of that and it's not necessarily for the woman's protection. It's the medico legal protection 
for myself that I'm considering. Because I'm pretty happy with what's going on. But if it's longer than normal or average or the medical how long it should be. Like we are not getting there at a centimetre an hour. But we're progressing. She might have quite widely spaced contractions so she's not going to get there so quickly.

\section{Group 5}

F1

I try to keep them at home in those early stages because I find when they come into a foreign environment the endorphins don't work the same. So you lose those hours and they're all counted aren't they? And they say 'six hours we'd better give her a caesar'. But she might have been only niggling. It's when you define when labour starts and they don't start labour until they're getting nice regular contractions.

These midwives discussed the risks associated with prolonged pregnancy and labour not so much in terms of risks for the baby. They seemed comfortable that they had the skills to manage these. The risks to themselves were of more concern.

\section{Being accountable - 'the eagle hovering'.}

The risks that the midwives themselves faced were located within the context of the medico legal environment and related to being accountable for adverse outcomes. For the midwives this was the most problematic aspect of practice. Only $25 \%$ of the surveyed midwives agreed that the medico legal environment made childbirth safer for women and only $28 \%$ thought that the medico-legal environment did not restrict their practice. The focus groups further revealed the strength of this negative attitude and uncovered the degree of anxiety related to the medico legal environment. Whereas they had spoken confidently about their skill in assessing and dealing with risks for the woman, it was a different matter when it came to dealing with their own risk. No midwife in any of the groups spoke about this positively. For them, accountability induced anxiety and sometimes dread.

\section{Group 1}

F3

I think about it all the time. A classic sort of thing that I think of often is, like on Friday night, you might be on call for the weekend but you just think 'oh I've got through the week and no work planned'. And someone rings up and says 'I didn't feel my baby move today or for two days' or something like that. Why didn't you ring me earlier when I was...

F2

At the hospital

F3 
Yes, when I was out and about. And there's almost this insatiable obligation that you must attend to this, that you have to go and do a CTG or give them a printout or whatever, to protect yourself. Yeah

F2

Yes. Whereas before in times gone by you may have said 'oh well just lie down for 20 minutes and see how many times the baby moves and give me a call back and we'll discuss it then' you know but ...yeah. It's a killer F1

I find that really interesting because I thought the older hands would have said just chill out. Whereas as a new practitioner I sort of feel obliged that I must do the CTG. I must check. I must do the bloods. I must go and find out or do whatever is indicated at the time. And I've sort of felt that I've been medicalised by checking everything so thoroughly. But it was an old hand who said recently 'cover your arse', you know. You've just got to do it. That was interesting. Yeah!

F2

I'd like to talk about that because for me, constantly in my practice I'm aware as I said of the eagle hovering, like ready to come and have a go if you make a bit of a slip or a misjudgement or you miss anything. And I think that that constant reassessment of what's going on, goes on even 30 years down the track, that you're constantly thinking 'are we okay here?' But I think it takes quite a toll. Because I think for me, and I would consider myself an experienced practitioner, I'm very conscious of that and it's not necessarily for the woman's protection. It's the medico legal protection for myself that I'm considering. Because I'm pretty happy with what's going on. But if it's longer than normal or average or the medical definition of how long it should be. Like we are not getting there at a centimetre an hour. But we're progressing. And she might have quite widely spaced contractions so she's not going to get there so quickly. So I'm reasonably happy that things are pretty okay for her. Her recordings are fine, she's making progress but it's slow. But I'm very conscious of what happens if I end up transferring in, or when do I decide to transfer in of what's acceptable in there.

\section{Group 2}

F3

Well I think the medico legal environment has made me a lot more defensive and probably I would refer sooner than I would have five years ago, before I was aware so much of the medico legal implications. I carry that on my shoulder all the time. What if, you know?

F2

Yes. I think [midwife] needs to expand on that though because she's been involved [ as an expert witness] whereas we haven't so we wouldn't have the same exposure.

Q

So medico legally, do you carry it with you all the time?

F2

I try not to because I know it will be that negative energy. But I am aware of documenting and if I make a decision then I need to document the whys and wherefores and all the rest of it. So if the medico legal thing 
happens there is a statement of fact for it to be referred back to. Having been involved in one ACC case, where the documentation was the one thing that saved the day. Everybody was aware of the situation and the facts and what was said and blah, blah, blah. That to me is the main point because I try not to think negative energy. If I carry the medico legal with me all the time I would practice definitely more defensively. So it's way in the background as much as possible. The documentation is there so that if I refer back, then hopefully it's covered.

There appeared then to be some dissonance between their attitude to the woman's risk and their attitude to their own risk; they seemed to be anxious about their own risk even when they did not think the woman was at increased risk. This was due in large part to the dissonance between what doctors and midwives might consider normal. The way the midwives discussed this reflected that they thought that medicine's view would be considered as authoritative in a medico legal context. This was further complicated by an impression that women expect a perfect outcome and that the midwives would be accountable for any outcome less than perfect.

\section{Group 2}

F2

They're shopping around. They're not happy to have an adverse outcome or even an outcome that is less than great. I'm talking generally because I know a lot of women are very reasonable. But there are more women who are wanting the perfect birth and they're expecting us to provide it.

There is then risk to the midwife on two fronts. The first relates to feeling accountable to the woman for any outcome that is less than perfect. The second relates to the way medicine has the power to define what appropriate midwifery practice should be, the authority that medicine has over midwifery despite autonomy. The midwives became anxious and cautious when they were working in ways that they perceived doctors might not approve of.

The midwives felt that risk was gaining increased importance and that it was affecting both the way they were practising and the nature of their relationships with women.

\section{Group 2}

F2

I see myself as in a much more risk situation in that relationship with the client than I did when I first started going to home births.... And now I feel like I'm removed because I'm protecting myself really with more documentation, more knowledge of what can go wrong... 
There was then a general sense of fear associated with being accountable. This was related to the processes involved if there was a negative outcome. The midwives did not seem to have a sense that the processes involved in being accountable would be fair or appropriate.

\section{Group 5}

F1

She used to take four patients a month because she had home birth and she said if two went to secondary care well that was her income. So she was reluctant to hand them over. But at the end of the day if you're standing in court, you're worrying about more than your jolly income aren't you? That's the last thing you'd be worrying about. I'm very mindful of that. Don't you feel like that? Why am I doing this? Actually I don't have to, so why am I? Because I love it. That's why. That's the risk, the risk to us. I've seen what they do to other midwives. They can't go out of the house for the news media, which is pretty sad. So that's why I try and keep on my tracks

The considerable amount of negative publicity received by a New Zealand midwife; referred to in the extract above ('they can't go out of the house for the news media') has left a considerable mark on New Zealand midwives. They have also watched what has happened to colleagues and are fearful of what might happen to them. Their responses: 'the eagle hovering', 'keep on my tracks', 'protecting myself', 'practising defensively', 'it's a killer', cover your arse' 'carrying on my shoulder all the time' are all comments that indicate the strength of negative feeling that the midwives experienced in relation to their own risk.

Given the extent of their anxiety it is interesting to try to establish how midwives manage to retain 'being with women' as the centre of care. The medico legal environment certainly seems to challenge this. This is rather paradoxical and reflects earlier comments about midwifery being both defined and not defined by risk. It is also interesting to note that midwives retain some sense of protection for the woman, sometimes at their own expense. The following extract reflects some of the different approaches that they use. The midwives in the following excerpt are talking about how they manage when the woman's pregnancy goes past its due date. One makes sure that the woman hears the viewpoints of all practitioners. The other attempts to protect the mother from anxiety by avoiding her having contact with opposing viewpoints. 
Yeah I had someone just recently who went up to 43 weeks. And she was told the risks by me and by about three different doctors, and she still stood there and I said 'oh okay well fine' you know. Because she'd had, three antenatal day unit appointments and the baby was absolutely fine.

F1

[Midwife's] saying that she'd get other people to talk with the woman. That's probably one of the most useful referral moments. If you haven't been convinced by the woman when she says 'yes I understand', that if she talks with someone else then she can never actually say to you 'I thought I understood but you were the only one who ever explained it to me so you must have not covered the points I really needed to hear'. I really like it if someone, and particularly if its someone from a different perspective talks to her. If she can still stand there and go 'Yes, I understand', then yeah, that's okay.

\section{F3}

I actually take the opposite approach. I think of the woman I had that went to 43 weeks. I kept her out of the hospital system. I wanted to protect her and we had talked about the risks of post dates after 42 weeks and I'd actually outlined the risks in her notes and she signed them with me. As far as checking the liquor volume and things, we did them through [private clinic] because I didn't want her to come head to head with an obstetrician or a registrar who would influence her and make her feel unsure of herself. So I probably tend to do the opposite. That was a homebirth and I and I don't know why I do it, but I know I practice differently with women planning to be at home birth. I guess its a different scope of practice.

Others also found it challenging.

F2

Women often don't realise of course at that moment because they feel what's going to happen to them, and we can see you know how difficult is will be. But you can't paint a picture of doom because we have to remain positive. But of course at that point we'll often say 'now you're going to need referral'. It may mean that I'm not able to be involved in your care. It sometimes has to be said.

F3

Yeah. I sort of think well now that that's happened I have to do such and such. I have to ring up and talk about it and sort of try and accept it you know because yeah I probably did used to practice a bit....

F1

...defensively

F3

You know bearing in mind that it could end up court if it doesn't 
Yeah, if something goes wrong and I haven't done what I am supposed to do, or I haven't done something according to the consensus, you know?

There was considerable indication in the both the survey and in the focus groups that accountability in the guise of the medico legal environment plays an important part in midwifery practice. Midwives are accountable for their care to the women, to their profession and therefore to the community. This accountability has become an important part of being a professional. This research sought to examine how risky this was perceived to be and found that it has a significant impact on how the midwives feel but not necessarily on how they act. Although several of the midwives commented that their rates of referral had increased over the past decade, the survey was not able to assess this. The survey was however able to ascertain whether there was any relationship between the midwives current referral rates and attitudes to the medico legal environment and found that there were none. The only differences that were found were that self-employed midwives were more negative about the impact of the medico legal environment than employed midwives. This finding seems understandable in that employed midwives could feel that they have added protection from the institutions that employ them and are less likely to feel autonomous and thus responsible.

\section{Working the system}

Understanding the context in which midwives practiced and being able to manipulate it for the benefit of the women was important in all the focus groups. They attempted to 'work the system' for the benefit of the women

\section{Group 6 \\ F3}

Yeah I must say being new back into the system, that it's the system that trips me up all the time because it changes and it is quite new. I always talk to [other midwives] or whoever else I know who is around who has been through it. You know just to say 'well what do I do in this situation?' Because I don't always know the system. It's not always clear who you've got to refer to, where and when. And while I haven't had a lot of women to refer, I have had one that I've had to consult about. So it was a case of figuring out who was the best to consult with and how to really work the system for the benefit of the woman in that situation.

The aspects of the system that the midwives felt they needed to deal were the nature of the relationships that they formed, particularly with the obstetricians but also with 
others in the secondary care environment. They also needed to work with the referral guidelines. Underlying this was the need to understand and work the power relations.

\section{Managing relationships - 'hoping like heck you get someone you like'}

Just as having good relationships with the women was important for the midwives so too was having good relationships with obstetricians to whom they made referrals. Most midwives (72\%) felt that obstetricians in their area supported continuity of midwifery care. A smaller number (64\%) also agreed to some extent that there was excellent collaboration between primary and secondary care (Fig5.14 and 5.13). Midwives who felt supported by their secondary services were also less likely to feel negative about the medico legal environment. Midwives with good support were less inclined to feel that their practice was restricted by the risk environment, and were more inclined to think that the risk environment made childbirth safer for women. It would seem then that positive relationships decreased the level of anxiety that the midwives experienced. The following excerpt is from a region where there was a high level of support.

\section{Group 5}

F1

No it doesn't make me do things differently but I did have a case where I was accused and I hadn't done anything. I just thought to myself 'If you had!,' Whew, dear oh dear'. It's not nice to get a letter from the ACC that you've being investigated because somebody's complained. And I could say that I just had obstetrician support. They couldn't have done better if they'd been God.

If the midwives knew the obstetricians and could develop good working relationships, this made it easier to 'work the system'. Many of the midwives discussed how important it was that they were known by the obstetricians and that trust was able to developed.

\section{Group 2}

F1

If they know that you're an experienced reliable independent midwife they'll give you far greater leeway than somebody who they don't really know or they don't really trust. So it's sort of a combination of many different factors.

Having good relationships was important, not only because the midwives felt safer themselves but also because this facilitated care that was appropriate to the needs of 
the woman. The survey revealed that nearly $40 \%$ of the midwives accompanied that woman to the initial antenatal referral in order that this might happen. Midwives who had developed positive relationships with obstetricians were 'allowed' greater autonomy in their decision-making. They were more trusted. In the two focus group regions that had most choice as to which obstetrician to use, the midwives could work the system in order to work with obstetricians with whom they had good relationships and whose manner and skills they thought would best meet the needs of the woman. The following extract from a rural area illustrates how midwives attempted to manipulate the system in order to get women to see an obstetrician who they felt would be most appropriate.

\section{Group 4}

F2

Yeah so you can pick the one that you know will be more open

F3

So that I can choose one that I have got a good relationship with. Or that I think I've got a good relationship with.

F3

Well yeah, an obstetrician visits [the rural clinic] once a fortnight. And we can refer women directly to see him. But it isn't easy to get in there. Like there's often a few months' waiting list.

F1

So there'd be people like the previous Caesar who would like to see an obstetrician.

F2

For long term things

F1

And of course the other thing is with that one is that he is very open. Well he has moments! But he doesn't push for them to go under the team. He'll work cooperatively with the midwives.

F3

And sometimes if women would like a referral not for a specific reason, I would put them through the antenatal clinic at [the rural clinic] rather than send them to [the secondary hospital]. But I don't think it's a good idea to be sending primips there any more because he's telling them they need to birth at [the secondary hospital]. So it's giving them a little bit of fear that they need to have the security of a big hospital around them.

F1

He's also telling them that $50 \%$ of primips need intervention.

F3

Yeah?

Q

Need?

F1

Need intervention of some sort, which you know compared to our rates here, is ridiculous. 
F3

And if they go to [the secondary hospital] often they're sitting round waiting for ages at the antenatal clinic and they're not very happy by the time they get back here. It's about an hour's drive to get up there so preferably we send them to [the rural clinic], which is where the obstetrician that we all get on with generally visits.

In the areas in which midwives had good relationships with all the obstetricians they had less need to pick and choose between obstetricians. There was considerable flexibility and openness in the interactions. It is noticeable that in the first of the following extracts the midwife could even consult with an obstetrician if he/she was not on call.

\section{Group 5}

F1

Good, no problems. It doesn't matter, like there's criteria to refer, which I do with everyone, even if I'm in doubt. I've got one woman at the moment with epilepsy. She hasn't had a convulsion, she said, for five years. But I'll still refer and they're happy with that because you're better to refer than not refer. And then they'll write back to me and say 'okay just carry on with the care' or whatever. I find that's good.

Q

And you get to speak to the obstetrician straight away or do you have to go through a registrar?

F1

No, the obstetrician if you want. They never turn you down. If it's just a query you go through the house surgeon. We didn't have a registrar. There's one just started. But [we refer to ] the house surgeon, if it's just 'when shall we induce this woman?' But if it was something you really want to know, you want to get to the source. And it maybe an obstetrician that's not actually on call today and he might be the one that's been calling the shots. And I try to go to him if I can. That's the one the woman knows and he's the one that knows the woman

\section{Group 4}

F4

We've got really easy access by telephone, like you can ring up any time and speak directly to the consultant and present the problem and you ask for advice and they will say either bring the woman over or 'I'll see them tomorrow at your own clinic' or whatever. But they're very very readily accessible at any time of the day or night.

Q

Do you think they're supportive?

F2

Very yes much. We have a very good rapport (General agreement). Compared with stories you hear from other areas.

F5

They kind of trust us and, and we've got a kind of rapport that means you know what to tell them. We don't want to take a risk down here. When 
we want somebody out in a hurry, we want them now. We don't want to wait an hour and they trust that we know what we're doing. We don't tend to have great delays here that much really.

\section{Group 2}

F2

For example I had a woman not so long ago. There was a very low lying placenta that was two or three centimetres from the os. The public obstetrician said 'Section her at 38 weeks. Book her now.' At this point we were only 36 weeks. And I'm thinking 'Well we've got a little bit of growing room here with the lower segment'. So I sent her to a private obstetrician who I know well and knew her background. She said 'No charge, we'll wait'. She has a straightforward normal birth. Okay she has a heavier loss than average, but far less than a Caesar and she's happy and her future has a lot less risk. And you feel she did a good job and doesn't look for any return except that the woman and the baby are fine and they've got a good outcome.

This contrasts markedly with the situations where the collaborative relationships are poor.

\section{Group 1 \\ F1}

We don't always get a choice. If it's an acute situation most of the private's [obstetricians] just won't come, even if it's during the day. At night it's the same. They're not interested. And even sometimes if you're consulted during a pregnancy with a woman seeing the obstetrician and then they have an issue in labour, you'll ring them up and they say 'Oh just hand them over to clinic'. Because even though that rapport has been established and often it's been established specifically if there is a problem, they want that consultant. They say 'she's just not my booking so I'm just not interested'. So it's very much a personality thing or I suppose it's their professional choice not to get involved

The importance of working with doctors with whom the midwives had a relationship was reflected in the way they talked about working with registrars.

\section{Group 1}

F4

It's always quite difficult when you're transferring someone in, in labour and you've got the new registrar. You know from what they're asking you, that they don't actually think that your skills are very good. And it takes probably two or three times to actually see that person again before they really think 'oh well perhaps she does know what she's talking about'. And they just let you get on with what you want instead of wanting to start at the very beginning and you waste a whole lot of hours. I don't see how you can really get around that too much unless they look at bringing registrars in on more of a rotation. 
F3

What about having consultants in the delivery suite? It's all that knowing again. You know, like when you say 'I need this' they'll just do it. But it's like that lack of consistency when new registrars come through. They say; 'Oh no, we'll do this and we'll do that'. And you think 'oh no, they're going to have you sitting here for another six hours. It's going to end up a section or the syntocinon. Its not going to work' But if I could have talked to a consultant it would be better

F1

Yeah they'll do what you want

For the new graduate midwives, working the system was particularly difficult, mainly because they had not yet been able to develop the trusting relationship with the medical staff.

\section{Group 1}

F2

Yeah, like when you've been around you can sort of see that this group of midwives tend to link to that obstetrician, and this obstetrician tends to group with those midwives. So it's pretty pre-determined. And I think where you see it most is for the new grads trying to link in and like, nobody is interested. And so often they fall back on having to use the clinic staff, which has a distinct disadvantage. The major number one disadvantage is the lack of continuity so the women see somebody different every time they go and get a different story.

There was some indication in the groups that easy and trusting relationships with obstetricians was being eroded by the anxieties of the medico legal environment. The ability to chat informally about problems or to ask questions was being eroded as doctors became increasingly anxious about what was referred to (in a derogatory sense) as 'corridor consultations'.

\section{Group 4}

F1

Yeah. But when we started doing that and we used to ring up and say 'Look I'm just letting you know that this and this is happening. So that if we turn up you have an idea of what is going on'. And we actually got into trouble. We got told not to do that; that they didn't want to know. That if we did that we were just setting them up for problems. They didn't want any of these 'we're just letting you know'. They feel they then have to do something about it, so they don't want to know, basically

This contrasts with the following excerpt from another rural group who felt they had free access to advice at any time. This group had good relationships with obstetricians. 


\section{Group 3}

F7

But they know us the moment that you ring. They say 'Oh alright, da,da,da, come over'. It's already set in place for us. It's not a hassle. The obstetricians know us don't they?

F1

Because we have thrashed this out with them. I mean we have discussed being in this area with the obstetrician. That it's no mean feat being an hour and a bit away from them if something goes wrong.

F1

But I think they've come to the party over the last couple of years would you say?

F4

So the support from the obstetricians and paediatricians is great. You can ring them at any time.

F2

Yep

Q

What made it good? How did you get it good?

F3

Because we're good (general laughter).

For this group of midwives being known by the obstetrician, even though they were in a rural area was crucial. They had gone to considerable effort to build the relationships. The midwives and obstetricians had come to know each other through weekly conference calls and two weekly obstetrician visits to the clinic where they also had a face-to-face meeting. These meetings seemed to have a high priority for the midwives and they came from all around the area to meet. They were on first name terms with the obstetricians and could deal with conflict constructively and assertively.

\section{Group 3}

F2

And then there are times that we get bawled out by them.

F5

Yes. But you can go back to the next day and go 'well, hey that wasn't really that appropriate' and they would go 'oh yeah okay.' Or there would be a discussion where they are not just a playing God. We go 'You listen to what I say!"

F4

And where there has been a traumatic time, they're very willing to sit down and debrief, the obstetricians and paediatricians. And they'll go through the case with you. I really think we're very, very fortunate with our medical staff. 
The midwives who had choice also spoke of matching the woman with the obstetrician. For these midwives the characteristics and perspectives of the individual obstetricians were important. Midwives in larger urban areas had the possibility of seeking a second opinion.

\section{Group 2}

F2

But again it would depend on the woman and whether she's comfortable with it. For me if you've got a woman who is a little bit on the back foot maybe, not used to dealing with health professionals, quite shy, passive and will accept anything that anybody tells her and you're feeling well, " $m$ m I don't think she'll get the best deal if she does that'. Then you might think about sending her to a particular private obstetrician who would be understanding of where she's coming from and would give her options in a way that she felt she had choices.

F4

We've got s an amazing amount of choice because if I don't fancy who's on as consultant today, I can send them over to [another clinic]. They'll see them three times in terms of the assessment unit without asking them to transfer back here. We're sometimes in a situation like if we're coming up to Easter, Good Friday, a Friday afternoon clinic is not going to be running. If I don't want to send her on the Wednesday because I don't like the particular consultant that's on, on a Wednesday because she's very interventionist, and I know she needs assessing I can send her over to [a different clinic]. And that's one of our options because we're only level one unit.

F3

Well I do get frustrated about the lack of consistency between consultants. The fact that you do have to think about who's on and the fact that you do choose to send somebody. I mean I have to say several times I've sent somebody to the satellite clinics where consultants visit from the hospital.. And having been given their opinion and their directions, I then thank them very much and send the woman for a second opinion to another consultant who I know will give a totally better option. And that's personalities and people's backgrounds. In [a sub region] we have visiting consultants to the satellites and they do change. They might get their area changed or they move on and do different things. So for instance we did have a very experienced person who has now been promoted and when we lost him we got somebody not quite as experienced. And her practice is totally different. So I mean I'm getting second opinions a lot more for her practice than I was for his and because we were on the same wave length, if that makes sense. And he didn't have rigid criteria. So that is frustrating and particularly when we lose the most senior consultants who are more flexible and relaxed it does make things difficult. 
The following extract is from a region that had had a comparatively poor rating on the scale of collaboration and reflected that even in one region the quality of the relationships and thus the ease with which the system functioned varied. When I asked whether the referral processes were working well the group responded:

F2

Which day is it Joan?

Q

So according to the obstetrician or according to the day makes a difference?

F1

According to the on call day

F4

It works as well as anywhere else, I mean

F1

Yeah but some places work exceptionally well

F4

But some, we could, if it's going good it works exceptionally well in this area but we all have hiccups.

F2

I mean because it's people we're dealing with.

F3

Overall I think we get a reasonable deal in this area. They can manipulate the system if they want to and if the midwife wants to work the system to get a reasonable deal. Occasionally it falls flat but

F1

And it's been changing too, it's been worse.

F2

Its getting better.

Q

What makes it get better for you?

F2

When there's more respect and negotiation

F3

And also you're not new

F1

And I think there is more midwifery voice presence now. Like even the, the hospital midwives are inviting us to their monthly ward meetings so that there can be more communication. I think there is a genuine effort to bridge that gap.

F3

I think the law changes made it better too. The law is on our side and they

try

F2

Which law is this?

F1

Section 88 
F2

Section 88 , that they've actively flaunted previously.

F6

$\mathrm{mm}$

F3

$\mathrm{mm}$

F2

And we have stood our ground and gone over their heads and it's worked.

It's good.

Q

And do you feel like it works well for you?

F4

$\mathrm{Mm}$

It was the relationships with the midwives employed in the hospitals (the core midwives) that caused the midwives in the focus groups the most concern. These were the only times in the groups where any participants became upset. Whereas difficulties with the obstetricians were expressed more in terms of frustration, difficulties with core midwives were expressed as distressing. The following excerpt is from a rural group who had previously spoken very positively about their relationships with the obstetricians

\section{Group 3}

F4

But things like, postnatally it is more of an issue there. Somebody for example has had twins at 34 weeks and babies are in special care. Then the staff will be ringing us now when are you coming over to do your postnatal visit and would expect us to drive an hour, do a postnatal check and then drive back again or just to have a chat. And the pressure comes postnatally. 'oh but she is your client'. And I would say that obstetricians are usually a lot more understanding and will often either offer you a bed to stay the night. But other midwives will just hang you out to dry.

F1

Horizontal violence with the midwives is just horrendous.

F2

Sometimes you feel like you're wanting to cry and you're just so unsupported.

F3

Yeah

F2

That's right.

F2

It's not all of the staff. But there's a huge element and that's the hard part I think. 
F1

I just feel that they're not getting any satisfaction themselves in the job. It must be boring. They're doing either an eight hour or twelve hour shift. And they just get so unhappy about it so they pass that unhappiness on to us.

Two examples where the core midwives worked well with the LMC midwives were both instances where the baby had died.

\section{Group 4}

F4

I had a woman recently, who rang me and said that she hadn't felt her baby move. And I asked her to come into the Centre here and there was no heartbeat and so we went up in my car with her to [base hospital] which in retrospect I don't know whether it was the right thing to do or not. But at the time I just felt a certain immediacy that we wanted to get somewhere. And unfortunately her partner wasn't with her at the time. But she really wanted to find out if her baby was okay. So I went with her in the car and I took her through all the processes in the hospital setting and then left her there and I came home. And the following day she decided to have her labour induced. And I worked in with one of the hospital midwives who I knew. I rang up and asked if we could do this together. And it worked out really well. She started off the induction and when she got into labour I went up and cared for her in labour.

Q

So when you can do it cooperatively like that it might work well.

F4

It worked really well.

Q

But that doesn't sound like it always works?

F4

It doesn't always happen like that.

F2

I had the same thing. The baby died and I went up. Oh she went under the team for an induction for the stillborn baby. It was right at term as well and I got to go up and catch the baby, inadvertently actually.

F1

It was supposed to be under the team but they all walked out.

F2

But they were really good and cooperative. Yeah because they knew it was the best for her, for the woman.

There did seem to be a general agreement however that there was a lack of support from some core midwives. When care became complex the LMC midwives felt that they often did not get the support they needed and found 'working the system' where core midwives were concerned much more difficult. This was also reflected in the survey. Less midwives agreed with the statement that there was excellent 
collaboration with secondary care services than agreed that obstetricians were supportive. Given that core midwives are the other group of practitioners in the secondary care service this indicates that relationships with them are more problematic. The midwives often found working the system where core midwives were concerned very difficult.

\section{Group 1}

F1

The [LMC] midwife often doesn't have the choice whether she stays on or not, if labour ward is busy. You have to stay on and do it whether you know what to do or not. And other times they [the core midwives] are bored and they'll go 'Oh out you go!' You know our own colleagues; our own midwifery colleagues can be very manipulative and unpleasant at times.

\section{Using the referral guidelines - 'whose permission do you want?'}

Midwives must deal not only with the people in the system but also the processes that the system puts in place. In this research these processes were the referral guidelines. The survey showed that most midwives $(81 \%)$ thought the referral guidelines were a useful tool when considering the need for referral but that $46 \%$ considered that they required them to refer more often than was necessary. This was reflected in the attitudes of the midwives in the focus groups.

\section{Group 2}

F2

I'm happy with the ones we've got at the moment. I wasn't happy with the proposed changes where every little iota was included. An SRM [spontaneous rupture of the membranes] of six hours had to be referred. They were some of the proposed ones and I know it because not everybody agreed with the proposed changes. We still have the status quo, which personally I'm very pleased about.

\section{Group 5}

F1

It doesn't matter. Like there's a criteria to refer, which I do with everyone even if I'm in doubt. I've got one woman at the moment with epilepsy. She hasn't had a convulsion, I think she said for five years. But I'll still refer. And they're happy with that because you're better to refer than not refer. And then they'll write back to me and say 'okay just carry on with the care' or whatever. I find that's good. I think we've all got our feet on the ground and we realise. Because you're at risk if you don't aren't you? And the patient's at risk too sometimes. I think you should cover yourself as much as you can. You know you want to do what's best for the woman. And what is best for the woman is the criteria that they suggest is usually the best. 
It was evident both from the survey and in the focus groups that the guidelines were seen as a risk management tool not so much for the woman's risk but rather as a tool for managing the midwives' risk. The fact that $43 \%$ of the midwives thought that they made referrals that they didn't think were necessary was reflected in the discussions with the midwives. For some there was a sense of loss of control once a referral needed to be made. They felt the risk of intervention rose.

\section{Group 1}

F1

But for me whenever you refer like it's the beginning of the downhill slope, even if you're referring somebody you'd actually felt quite competent to cope with yourself. Like maybe a previous section. But the system points in the direction of doing consults for women with previous sections and so you do what you're meant to do and yeah sort of being a bit of a down hill slope really

Although many of the midwives spoke about the negative consequences for the women in having unnecessary referrals they had little hesitation in making them. There was also some willingness to provide care that went outside the guidelines, that is, not making a referral when it was recommended, if the women did not want to. In essence they remained women-centred in their approach. The guidelines then provided a parameter from which the midwives could measure their care. When they moved outside the parameters they had to take added precautions for themselves.

\section{Group 3}

F5

If I have a woman that is acting differently from the recommended best practice, I make sure that I address that several times. That it's not just a one off. And it's very simple. Just going back to it and saying now we talked about that, are you still comfortable with your decision at that time. Do you want to talk about it any more? So that in my notes it is recorded that we have addressed it and revisited that. And then I'm really happy with going outside that wall with the woman as long as they..

F4

You really have to start uncovering who the woman is and actually dare her to meet you in there, you know. That you're not just going to go out there and stand there all by yourself. And you say if you're going to stand in this space I can stand with you but I'm not standing out here, you know I'm not bringing my sense of what should or shouldn't be done here and you're standing somewhere else. Like if she says 'I had a previous caesarean section, can I have my baby at home?' I say 'when you ask me can, who's permission do you want? You know. What I want to know is do you wish to have your baby at home and how strongly do you wish it and what preparation of your mind have you done? Because 'I know about that, but you need to know about it too'. I'll say 'Sure let's do that'. It depends on where she's looking for her strength. If she just wants to 
take all of mine. But if she's going to bring her own to it, then my job is to support her in that strength.

\section{Group 3}

F5

Sometimes it means you have to stand up to people. You know I get growled at a bit by obstetricians for not doing guidelines. But I don't care because I've given the women the options, told them the research base information which the obstetrician's guidelines aren't adhering to anyway. So I don't really give a stuff. Yeah.

\section{Group 1}

Q

What about when the referral guidelines say you need to refer but you don't think you need to?

F1

Well for me that happens a lot and that's that medico legal thing. Like the previous sections for me is a very typical example, particularly someone who has maybe laboured and birthed with no problem. And still in theory you're meant to do a consult. And you know I'll talk with them about that and say 'well you know the book says that you have to do this'. And some of them say 'oh no I'm fine'. I'm happy with that. I mean I guess I quite actively encourage them [to have a referral] purely from a medico legal point of view. That if something untoward happens and I hadn't done that or suggested that she do that, then you know I'd be very vulnerable. So it's a self-protection thing. And yet it's stupid because it's just a waste of money and time

F4

Because it's a bit like multips and grandmultips. Some of them have very quick labours and no problems. So you think, oh you know. And some of them will say 'no I don't want to do it'. And I think as long as you have talked to them and given them that choice then it's okay.

Given that the development of the guidelines was a collaborative endeavour between midwives and doctors, there was surprisingly little ownership of the guidelines as being midwifery-led. They were seen very much as an imposition and as being driven by medicine. Understandably the newer midwives referred to the guidelines much more often than the experienced midwives.

\section{Group 1}

\section{F2}

Well as a new practitioner I carry it around with me (laugher) and just to know what I'm looking for. And then I'd go 'oohh do we need to refer?' and 'yes we do have to' and 'yes we definitely do have to'. It's all fairly clear. It's actually been quite useful as a new practitioner having that very clearly there. 
There seemed to be some variation in how midwives viewed and used the referral guidelines. The survey revealed that midwives who thought that the referral guidelines required them to refer more often than necessary also thought that their pattern of referral was less likely to be appropriate to the needs of the women they cared for and that they were not useful tool. These midwives, although in the minority, were also less likely to work in an area where there was excellent collaboration between primary and secondary service and were less supported by obstetricians. This raises the issue of whether these midwives varied in their approach to risk and referral, having different thresholds and different approaches to compliance. Similar correlations were also found when midwives were asked whether their pattern of referral was appropriate to the needs of the women they cared for. It would seem, given that the referral rate was not related to these attitudes, that there was compliance on the part of some midwives. They did not feel that they worked in a collaborative environment and referred reluctantly. This leads us to ask why they might do this and leads to the next theme, that of power relations.

\section{Power and authoritative knowledge - 'it's like the jungle'}

Much of the discussion presented in the excerpts so far reveals the interplay of the different power relations inherent in the system. Working the system also means working the power relations. Power play was not isolated to interactions between midwives and doctors. There are power relations between the midwife and the woman, between the midwives themselves and between midwives and obstetricians. There are power relations 'over', 'between' and 'under'. The following excerpts illustrate how midwives acknowledge and deal with their power over the woman and how they mediate power between the woman and the system.

The midwives had considerable power over the woman. The midwives acknowledged this, and attempted to manage it with a rather paradoxical sense of 'protective empowerment'. In a sense the midwives felt that they knew the system better than the woman, and needed to support and protect the woman's journey through it. 


\section{Group 1}

F4

Sometimes I think with that it depends on the woman and how forceful she is. Sometimes they'll go to clinic and they're getting passed around a bit and then she'll say 'I want to have a midwife from here' and suddenly they provide someone out of the air. Whereas normally if they're a little mouse and haven't got the confidence to do that and don't know how to question things they just get ridden over. And they just say 'okay you're going under the clinic' and they think that's what they've got to do. They don't realise that if they actually really put their foot down, they'll probably get someone from the teams that will look after them. And I've got into the habit now when we transfer someone over antenatally, I actually tell them to do that. And it's working. Because we had a girl with a prem, [premature labour] and was small for dates [baby not growing well]. And for a start they said 'oh she'll just have to go to the clinic'. And I said 'No, say that's not good enough, say you want your own midwife'. And anyway she did. And she rang me back and said 'Yes I've got someone'. And sometimes I think, because a lot of the team have people that are high risk and they deliver early and then they find they have got space but they don't get them passed on. They're quite happy to pick people up. But sometimes the clinic itself doesn't actually keep on top of that. Yeah I think sometimes when you're working in the system like that you do learn to find some solutions and manipulate it a bit. But it's not ideal

F3

And a lot of that is working behind the scenes, giving women heaps of information, just really priming them

F4

Yes

F3

And now 'If you do this, this is what will happen and it might be out of your control but you have a choice'. You work really hard sometimes trying to just give them that confidence I think.

\section{Group 1}

F1

I think the women lose any sense of control and the midwife has very little input. Well, might have the input, but has very little influence when that happens. Unless you've got a woman who's very clear about where she's going. Most women will just be overwhelmed by what the specialist says and so lose control over what's happening from that sense. They're not necessarily consciously losing control, but you stand on the sideline and see that they lose control, that control is taken away

\section{Group 2}

F4

And I think if the midwife's there, then for the woman there's more of a 'well how can we do this that it's best for the woman?' because we know it's got to be done and we know that we can't continue as we wanted to, 
as she wanted to. But maybe she can still feel like that she's a part of this and not 'done to'.

The midwives also spoke about times they needed to take control.

\section{Group 4}

F1

I had a woman who was bleeding at home. I said 'I think we should be thinking about going to hospital'. And she's saying 'I don't want to. I don't want to'. But you know, 10 minutes later I said 'Look I don't think we have any choice any longer, you know we've got the drip up, we've given you these medications, you're still bleeding, the recordings are not good da da da da. I think we have to go'. And she said 'Oh okay'. So it was the same thing. I was really bulldozing her into it. Although I think she had got to the stage where she was ready.

\section{Group1}

F4

I do say to the women though. I say 'I won't discuss all my worries with you and especially probably pertaining to labour'. But I say 'If there's an emergency and I tell you to turn over or we're doing this, that's what we're going to do. Because I've thought about it in my head and we've got to this point and my contract with you is that if we reach that point, I will tell you and we'll do what I plan. I may not have time to explain it to you but I've done a lot of thinking about it in my head before we've got to that point'.

Q

So an informed consent and choice, in reality, what do you think it's all about?

F4

Well it comes back to protecting them. But there would be an explanation later

F1

It's interesting isn't it? Because tell them the other way round. I certainly don't worry them with all my 'what if's' or we'd never get anywhere. But I say to them 'It's very very rare to have to make a decision right now. That most times you've got some time to consider the pros and cons of action and then make a decision'. You know, you were saying you prime the women up before they go and see a consultant at clinic. And that's what I'll often say to them. 'Don't be bulldozed into something because so rarely does a decision have to made now, even if it's two or three minutes. You've usually got that time. But you've even got longer most times' And so tell them to say 'I want to talk to my husband about it before we decide what to do'. 'You take the power before making the final decision. Listen to their recommendations and the pros and cons. But take some time out together to make sure that you're happy with that and then go with it or not'. So I mean I always do the opposite. Keep saying 'I want some time to think about it or I want to discuss it' or something and make a space for it. 


\section{Group 1}

F1

And you can shift them. Because some women will come to you, particularly the primips [first time mothers], and they want to see an obstetrician just to reinforce what you've said, basically. They'll come to you and say 'I'm going to have the baby at [secondary hospital] and I have to see a specialist because it's my first baby and I'm 34 and I'm old' and all these sorts of things. But if you bide your time and you work with them they'll end up birthing at home. So they can shift when they get some options and reinforcement that what's going on for them is normal. And okay, so how do you define normal? That's the problem you see, once again it's this black, white, grey thing

\section{Group 4}

F4

You know some of the informed consent that I give people is loaded a little bit because of my vibes. Because I really don't want to be stuck in a situation here in [rural area]. So I probably emphasise the risks a lot more than the benefits. I wonder where there is pure informed consent (laughter of agreement). And anybody that says they are pure, I would really question which side of purity they're on.

One of the most significant aspects of current power relations between midwives and women is the nature of the power that women have over the midwife in the form of accountability and the requirements for informed consent. The midwife then has both power over the woman in that her knowledge is authoritative but is also subject to the power of the woman. What is also revealed is that despite being autonomous practitioners, many midwives felt that they remain 'under' the power of obstetrics. For these midwives, obstetricians hold the authoritative knowledge which makes the midwives more vulnerable to risk. One of the main difficulties spoken about in all the groups was the lack of recognition by the secondary service of their expertise and knowledge. For the midwives at the interface of primary and secondary care there are often difficulties in asserting their position. Many felt that their own knowledge was placed in a subordinate position.

The way the midwives felt about the guidelines was reflected in the way authoritative knowledge was played out. The guidelines were often viewed as something that the obstetricians imposed rather than as a collaborative relationship with midwives. Working outside guidelines that authoritative 'others' had imposed made them vulnerable. The authority of the midwives as experts placed them in a position of being in between. As knowledgeable professionals they were experts in their dealings 
with the women. Yet when they came in contact with obstetrics their knowledge could be seen as inexpert, as less authoritative. The midwives felt that it was important to have their knowledge and skills acknowledged. They expressed varying points of view about the respect their knowledge received from obstetricians.

\section{Group 1}

F1

There's no respect that the midwives actually know what's happening, like they know in every sense what is happening and what comes across clinically. They may say 'Oh well, she's only been labouring for $\mathrm{x}$ number of hours or we've got to push the synto for a number of hours'. And you know they haven't got the feel of the labour or what's happening or what's not happening which is more important. And you know inevitably. I mean it's very rare to be proven wrong. And you get to a certain stage where you know that this is not okay and this is what you need to do, or what needs to happen. And nearly always you're right. But often you have to go through a protracted process. Also, the other way around. You think 'No we just need an epidural and some synto and we'll just get things moving along a bit more, we'll get there'. Because you know what's going to happen. And the frustration is that we don't have the say-so to say 'Well this is what's going to happen'. Sometimes we do because we have obstetrician colleagues that we know or are prepared to keep us quiet (laughs) and go with what we say. That's what makes it easy. But I think it's actually the frustration of having a really good idea of what's happening and not being able to get that message across and get it acted on

In the interface between primary and secondary care there was always the possibility of loss of control.

\section{Group 2}

\section{F1}

It's not that I'm like a mother lioness over cubs or something trying to defend them from other people, but I know that there's going to be input apart from my own and I can't always trust that and I think that's where my grief comes from. That I have some very unreliable obstetricians who will fill their heads up with all sorts of rubbish and I have other ones who are so good, who give support and still try to stay on the normal track. And there's this sort of huge gap between the two and I don't feel comfortable because I can't guarantee that we're going to get this one. It could be that one or it might be she's being counselled by this one and seen three or four times in clinic by this one. And then on the actual day it turned out to be that one and I think 'oh shit'! You know, it's just loss of control. 
The following excerpts are examples of the place authoritative knowledge plays in decision-making. In defining the length of a labour medicine has the authority to say how long a labour should be and this can differ from the midwife's understanding.

\section{Group 1}

F2

Because I'm pretty happy with what's going on. But it's longer than normal or average or the medical how long it should be. Like we are not getting there at a centimetre an hour. But we're progressing. She might have quite widely spaced contractions so she's not going to get there so quickly.

\section{Group 3}

F1

And [midwife] went through her access agreement the other day and what did [the obstetrician] say? 'Now when you have someone who's not making progress, you get them up here sooner rather than later'. And this is drummed into everybody. That you don't wait more than so long for a multip and so long for a primip before you transfer.

F2

Yeah that's where you're going outside the medical profession's terms of normal. So that's where you take risks.

F3

That's right.

This excerpt also reflects how the midwife will manipulate the system. She keeps the women at home so the hours don't get counted by the 'they' who will 'give her a caesarean' after 6 hours. Interestingly this participant was from the region with excellent midwife/ obstetrician collaboration. Even here there was a difference between midwifery and obstetrics about how labour might be measured and also a sense that it was the obstetrician rather than the midwife who had control of the decision-making. The trick for this midwife was to keep the obstetrician out of the picture for as long as possible.

In order to hold some authority in decision-making the midwives needed to be known by the obstetrician.

\section{Group 2}

F1

If they know that you're an experienced reliable independent midwife they'll give you far greater leeway than somebody who they don't really know or they don't really trust. So it's sort of a combination of many different factors. 
There is interplay then between power and personal knowing; the relationship between power and connection was important. The obstetrician had the power to give the midwife leeway. The midwives valued the relationship not only because they could learn which obstetrician's perspective they trusted but also because they could then be given leeway. There was no evidence in any of the discussion where the reverse was true; the midwives did not have seem to have overt power over obstetrics. Power in these instances was covert. They needed to manipulate the system to assert their positions.

Although much of the discussion reflected this power imbalance there were examples of midwives claiming their own power within the relationship. Some spoke of the importance of acting confidently and with authority.

\section{Group 6}

F1

I reckon it depends on how you approach it. I think if you ring them and you've got all the facts and you're confident when you're talking to them, they're fine usually. You know what I mean? I think if you come across confident they're fine. Even in delivery suite, if you're ringing them about someone who, you know, the foetal heart's dicky or whatever. That's just my experience with it. I mean sometimes I think perhaps it's our ability to cope as well. You know, like if you get a response back that's not that great, you can actually sort of counteract it better. You know what I mean? You're not as vulnerable. So I think a lot of it is that if you're feeling a bit more vulnerable. I think, it's like the weak in the litter. They can sort of hone in on that a little bit if they feel you're a little bit hesitant or something like that.

F2

It's like the jungle.

F3

I think a lot of it is psychology.

F1

I found when I was new. Like lots of people think like I'm really confident and I don't feel confident at all. But I found even like I'd say to them, I'd just say how it was for me too. Like say 'actually I'm feeling quite scared. I'm not sure what to do'. And they're quite responsive to that. They don't kind of shoot you down. And I always think it helps when you're bigger than them too. They don't get so stroppy

F2

I think you have to appreciate their side of it too. Like especially with referring in. You know if you've got a woman in labour and they walk into a situation that we can no longer handle. They've never met this woman before and we expect them to, you know, PPH [haemorrhage] or 
foetal distress or something. You do have to always appreciate their side too.

F3

Respect has to go both ways for it to happen.

F2

Absolutely.

F1

And for them trying to get all that information about the patient like that. F2

And they always walk in, in either an emergency situation or something that's completely out of our hands

F5

Yeah exactly

F1

$\mathrm{Mm}$

F2

But we always stay there and bridge that too.

F2

Oh yeah, I think the issue comes when, when the advantage which they might have in our presence is negated and, and they don't have a lot to go on.

\section{Working with complexity}

There is much that is unclear in midwifery practice. An examination of the place risk plays reveals its complex and often contradictory nature. There can be no simple explanation of how midwifery works. So far I have shown that midwifery claims a position 'with women' within a professional relationship and that it does so based in a system of maternity care that needs to be manipulated or 'worked' in order to do so. The data revealed that this is no simple task and that there are many contradictions and paradoxes involved. So much so, that a theme emerged of its own to reflect this.

\section{Being normal - 'excluding everybody that doesn't fit into the middle'}

The place where this complexity is most clearly revealed is the place where 'being normal' was defined. As previously stated midwives claim expertise in normal birth. However midwives interpreted normal in a wide variety of ways. Normal was discussed in terms of both process and outcome. Mothers, who had very complex pregnancies with multiple interventions, could have a 'normal birth' as long as the birth was vaginal and unassisted. Other midwives said that a true normal birth could only take place at home. Occasionally the midwives would contradict themselves as normal was approached from differing perspectives. Normal for some of the midwives was such a complex phenomenon that it seemed sometimes to lose any 
useful application. This perspective is aptly described by two midwives who were new to practice.

\section{Group 1}

F2

Yeah, I found I had to redefine normal when I started practice. Because I had a concept that you're physically well, that you're emotionally stable and that you're financially or economically stable too. And that was normal. Now what I found in reality is that there's always one leg missing on the stool. There's always a leg missing. And I had healthy strong women with absolutely no money and no partner or he's a crim. It just was awful. And that was normal for her. And then I had really stable relationships and financially okay and everything is hunky dory and then physically, bang, we've got something wrong. So it just seems to me every single client has one leg missing on the stool and that to me is now normal. And that's life. That there are problems and that's normal. So you know that's kind of my picture now is that you're bloody lucky if you get all three at once.

The following except shows that for this rural midwife, new to practice, this confusion about what is and is not normal can be threatening in a medico legal sense. She has thus tried to avoid looking after women who require secondary care yet had seen the difficulties of juxtaposing normal and complicated

\section{Group 4}

F3

Yeah, I did too [used to look after higher-risk women]. But less now. I'm sort of screening people. Yeah because I don't like going up to [secondary hospital]. The other thing is about direct entry practitioners. We had it drummed into us over and over again 'you are practitioners of normal birth', you know. And then the third year most of us were scared out of our wits because that was the year of doing complications.

F2

And somebody went to court of course

F3

And so I was quite fearful when I graduated. And then when you start getting your client load together you think 'Well this isn't normal, that's not normal, that's not normal. They didn't tell us this'. And you think you know 'what am I doing?'

F2

Yeah, very frightening.

F1 (experienced midwife)

Because it's normal for people to have things wrong with them. It's normal to be not normal.

For the more experienced practitioners the idea of what was normal in women had become expanded as they challenged current constructions of normality. Normal also 
has strong associations with birth outcome. For many this meant an unassisted vaginal birth. Where intervention was required they believed that the women could achieve a normal (meaning unassisted) birth. What could be considered a normal process could be different away from the secondary service, away from the medical gaze, especially where this related to the length of labour. There was often an underlying anxiety about being 'hung out to dry' if transfer to secondary care was required after a long first or second stage. There was therefore conflict between the medical and midwifery understandings of normal in which the medical understandings were considered dominant. When they provide care for women outside what is considered acceptable practice therefore the midwives expose themselves to risk as this discussion with a group of rural midwives shows.

\section{Group 4}

Q

So do you take risks in your practice?

F2

Every day

F3

Yeah

F1

Yep (General agreement and laughter)

F4

Life is a risk.

F1

Remember [midwife] and I had primip [first time mother]. She had a [pre-existing medical condition] as I said before. And this primip took three hours in second stage. She was on the bed here and we both sort of scuttled out to the other room and said 'should we take her through [to the secondary hospital]'. 'We'll just try, we'll see if we can get her into a squat position'. We go back in and she'd changed position and we get a bit more progress. 'Alright we'll wait a bit longer' and this went on for three hours. Finally we persuaded her to actually get her into squat and she had the baby no problem at all.

Q

Do you have someone sitting on your shoulder?

F1

Look if it hadn't been the two of us bolstering each and other and saying 'It's normal for a primip. She's making ongoing progress. The heart rate's fine, her recordings are fine'. You know we really had to bolster each other didn't we?

F4

We use midwives a lot

F2

to reassure yourself that it is a normal or

F4 
if we're concerned we use each other a lot if we're concerned about something.

F1

We also offered her the chance to transfer and she refused several times.

F4

And I mean, everything was normal! (laughter)

F2

We weren't worried. The foetal heart was fine, she was feeling really positive that this was where she wanted to have her baby.

Q

But why did you give that as an example of an 'at risk' situation?

F1

It was an example of a situation where if we had gone up there with a woman who had been three hours in second stage we would have had the book thrown at us.

F2

Yeah, because that's spelled out.

The risk/normal dichotomy was therefore blurred as midwives perceived that women with increased risk, both social and physical could be considered normal and could have normal birth outcomes. Normal became a problematic description. It was appropriate therefore that midwives did not attempt to make a distinction on the basis of what was considered normal.

There was a general feeling that normal birth related to a lack of intervention, but even then women could achieve a 'normal' birth. Midwives then had an important role to play in protecting and promoting normality. Normal was paradoxically both important and irrelevant. It was felt by many of the participants that the definition of what was normal was very complex. This extract of a group discussion illustrates this.

\section{Group 1}

F1

I agree very much with [midwife] that the woman's expectations of herself have a huge impact. Like this woman I was telling you with the kidney issue. You know, she was determined she was going to have a normal pregnancy and a normal labour and birth and it didn't matter that she had all these blood pressure problems and proteinuria and had a baby with problems and everything. She was going to do it herself and she did. But it took a lot of work because we still had to interact with the obstetrician who when her blood pressure was up and her blood tests starting going off and all this sort of stuff

Q

What's normal in that?

F1

Yeah well you know I mean the woman. She still felt fine. She was normal (laughter) 
And she wasn't going to be induced again. Because she was induced last time and it all went to jelly. And she had no control and she had crash section and da da da. I was actually happy supporting her with that, in that, you know I was sort of edging. It was certainly very grey, merging on black. And I think the more you work, the more you see that huge range of normal. Like my definition, if I could give it a definition. My understanding of normal has got bigger and bigger as the time has gone by. Not only from maternity care point of view. You know, like how long is a normal labour. That's certainly changed and how long is a normal second stage and those sorts of things. They have all expanded. But also what's normal socially. I can think of one woman who was on her sixth child, all to different dads. Some of the children were with her, some not. Like the first time I met her I could just not get my head around all the children that were there. Because there were some of her children and there was some children from almost every partner she'd had. But they weren't hers, they were his. But she still had them. And then there were foster children and I just couldn't get a handle on it. It took me about three visits to work out who belonged to what. And you know she was quite a heavy marijuana user. She smoked 50 plus a day on a good day. I mean she just smoked continuously. She cut her alcohol out for her pregnancies. You know CYPS [child welfare] were in and out, the policeman was in and out, the fella's were in and out. The incredible thing was to see her labour and birth. Exactly the same thing happened that happened at all her antenatal visits. When you went, there would be a different person there, or people. Very rarely one person every time. And they would stay and she would hold court. And you know we'd talk about everything, and exactly the same thing happened in her labour. And it just blew me away really, but it was normal for her. The drunk neighbour came in even though the mother had said that she didn't want her there. And we managed to send her away to find the daughter who was meant to be there. And I said to the woman, 'Would you like me to help you get the drunk lady away?' 'Oh no'. So she parked herself there. There was a collection of people who came and went exactly as in the ante natal visits. And it was exactly the same for her birthing. And that was normal. And like, how can I define what's normal? That was normal for her and so...what's normal?

\section{Group 4}

I think it's interesting what [midwife] said about normalising the abnormal. But we've got to look at the fact that a lot of abnormal is actually normal that has been abnormalised. And one of the things that we have done is we've actually cared for a lot of high parity women. We had a woman having her 12th baby here. She was actually booked for [secondary hospital] but we did end up looking after her. We've done home births with ninth babies and eighth babies and that doesn't bother us at all. We don't actually consider that abnormal, providing the woman is in good health and has a good obstetric history. So it's actually not why 
are we saying this is abnormal. We've actually got to look at the whole woman and not just one facet. So with things like parity and high age you know 40 year olds, 38 year olds. If the woman is fit, healthy, doesn't smoke, the baby's growing well then what's abnormal?

\section{Uncertainty -'that big grey area'}

It seemed that for the midwives there was a space in practice between the assessment of risk and the decision to act, where the complex nature of the work and the multiple and often conflicting claims made decision-making unclear. There was for many of them what was referred to as a 'grey area' of practice in which uncertainly often lay uncomfortably.

\section{Group 1}

F1

And I think there's a huge area of grey. And the more I've practised the more grey there is which makes it harder. Because you're always on edge, making the decision-making, making your decision. Whereas I almost envy you [another participant]. What you're saying that you've got fairly clear boundaries of when to bail out. Whereas I haven't got that. I've got all this space in here. I'm swimming around really thinking 'Now am I okay, can we keep going, what if she does this, what if she does that?" And I find the grey area is the hard bit. I mean the black and white are easy, but it's that big grey area and the more I practice the more grey there is.

The grey area is the space where the midwife felt particularly vulnerable.

\section{Group 6}

F1

Yeah it's really listening to the woman. Because you sometimes get double messages. 'I'm really worried but no, don't come'. And you think don't come but do come! 'I don't want to inconvenience you but you know I just had to tell you' and you sort of... 'Oh, but it's that really listening past the 'no don't come, no it's all right. I'm sure it will be all right until tomorrow, I think'.

The midwives often stayed in the grey area to protect the woman. It was often an uncomfortable and risky place to be and involved maintaining some balance between protecting her from anxiety and providing information so she could make choices about her care. In this area they talked about the importance of having each other to discuss things with.

\section{Group 4}

F1

Look if it hadn't been the two of us bolstering each and other and saying it's normal for a primip. She's making ongoing progress, the heart rate's 
fine, her recordings are fine, there's no, you know we really had to bolster each other didn't we? It was really...

F4

We use midwives a lot as, as

F2

to reassure yourself that it is a normal or

F4

if we're concerned we use each other a lot if we're concerned about something.

F1

We also offered her the chance to transfer and she refused several times.

F4

And I mean everything was normal, (laughter)

This grey area was the space in which the midwives took risks. Taking risks for the midwives was usually expressed in terms of balancing the needs of the woman with the demands of the obstetricians. In this place the midwife was clearly in a space 'between'.

\section{Group 2}

F2

I'm just thinking. My tendency is that when I can see that things are perhaps going a little bit off the normal road, I find it very hard to actually go down the referral path. And sitting for a while and watching and waiting might mean that what you thought was going to happen doesn't happen and it's all totally normal. But it can also mean that you delay in investigating at the clinic. And I just find that place is so uncomfortable to be, where I'm carrying that anxiety and waiting because I don't want to worry the mother and wondering whether it's time to intervene or whether I should just wait and see a little bit longer. You know, maybe in a labour where the progress is slow or I'm at [primary birthing unit] and I've heard a decel [fetal heart reduction]. But the woman's going to deliver soon. Or there's a little bit of meconium. That's really hard to carry and yet I'm afraid if I jump in too soon with the intervention...

F3

Yeah I guess I would have to admit to taking risks at times. For instance if you don't see any liquor you can assume there's none and perhaps there's some meconium going to follow the baby. If it's going to deliver fairly fast at home. Or you might be expected to transfer to be practising faithfully to the guidelines. But I would take the risk in the absence of any foetal heart abnormality.

\section{Group 1}

F1

Like this woman I was telling you with the kidney issue. You know she was determined she was going to have a normal pregnancy and a normal labour and birth and it didn't matter that she had all these blood pressure problems and protein and had a baby with problems and everything. She was going to do it herself. And she did. But it took a lot of work because 
we still had to interact with the obstetrician and her blood pressure was up and her blood starting going off and all this sort of stuff

Q

Yeah what's normal in that?

F1

Yeah well you know I mean the woman, She still felt fine, she was normal (laughter)

F2

That's that being strong

There was uncertainty in risk itself. It was often unknown, unseen and hidden in the grey. Midwives needed to be prepared.

\section{Group 6}

F4

I've been very aware of the whole risk environment and so even for practising but also being an educator. I really have to weigh up for myself what risks do I put women in because of my own busyness. And therefore what risk do I put myself at? But at the same time I put in place measures that actually reduce that risk. I may not talk about it in terms of 'This is my risk management practice', but it is an awareness that I do not want to create risk, purely because of my own busyness. And I suppose I'll always go into a birth situation in preparation with 'How am I going to deal with something if there is a post partum haemorrhage, if there is shoulder dystocia, or if I have to do resuscitation. You know it's almost like I go through the mental checklist of making sure I've got it all sussed in my mind. So you know in a sense that is risk management, but it's just practising safely really.

\section{Group 3}

F6

Well I always look at 'okay here we are'. I always keep an eye on the potential

F2

Yes as long as you explain it.

F4

Yeah you know like the

F1

You expect the normal but

F6

I expect the normal but I'm always on guard just in case. I keep a scenario of the worst. I keep a look out on what could possibly go wrong. But even in normal births I go to a level of stress and I don't relax until the hour after it. I'm always on a stress level. Like I said you're on the alert and the adrenaline goes, your senses are going. That's normal stress. But then you go on extra stress when something goes wrong. I don't relax F1

have a heart attack

F6

Yeah and I don't relax until the baby's checked, two hours after birth. 


\section{Group 2}

F2

The more experience you have the more, that grey area is so variable. So I can see that the more cases you have and people the greyer things get. This is a very humbling profession. People and things don't happen the way you think. On the other hand you can be very pleasantly surprised.

\section{Group 2}

F2

I can think of one situation where I had a student midwife and she did take me aside afterwards and ask me to go through step by step my decision processes. (laughter) I thought 'what have I done?' Well it was a VBAC [vaginal birth after a caesarean] situation that was planned for hospital. It was a very educated woman who'd done all the research and then had sat down and debated with her obstetrician what she felt would be best for her. So she was a woman who knew what she wanted. And obviously I think in hindsight deliberately didn't call me too soon. I visited her at home and she was nine plus cms with the head at +1 . And I thought there's no way I'm going to get this woman to hospital in time. However an hour and a half later, having pushed for an hour and a half, she was still at home. The student was thinking 'Well you could have got there'.

\section{Group 1}

F2

Yeah you go by the guidelines and you stick rigidly to them and you're paranoid to the hilt. You're really watching, really really being vigilant and just (sighs). I was at the low risk unit the other day and all the time I was going Well, are we okay?' And just always always always 'Are we still okay, are we still okay? I mean you'd be doing that anyway for sure but it was even more. That sense was really heightened because we were in a primary unit rather than in a base hospital, knowing that if things went into that grey zone, then when were we going to move. It was just much more heightened. Even making the decision to go to the primary unit in the first place. We had ruptured membranes and there was that real grey zone, 'Have we come up to the 18 hour limit?' That was really annoying me, having to decide and having to talk it through.

\section{Multiple world views -'Between a rock and a hard place'}

The midwives in the study were acutely aware of the wide variety of positions that people took. There were many points of view that needed to be taken into account: the mother's, the doctors, and also the prevailing culture of birth. She also needed to consider her own perspectives. In providing care the midwives often seemed in a position 'between' the worldviews of others where she was required to act as a mediator.

\section{Group 3}


F3

Yeah it's different because, it's different for obstetricians and it's different for us. And yet the two are somehow sort of linked. Because when you've got a complication you've got to refer someone. It's sort of linked into their way of doing things. So it's two different things and sometimes it's hard to sort of reconcile the two. And the other thing is we have the relationship with the woman whereas the hospital don't. It's difficult. You've got to see things from I suppose everyone's point of view.

In planning care with and for the women it was important to come to some understanding of where the woman came from. The woman's worldview impacted on how decisions were made and how action took place.

\section{Group 6}

F2

I often ask them about their decision making processes and how they make decisions in their ordinary life about things. And how they might make decisions as parents. And that this [the decision whether or not to give Vitamin K to the baby] actually may be one of the first ones that they will be making. And whether they like to make decisions that are based on research or decisions that are based on their life philosophy or decisions that are based on doing something to protect their baby, or doing something that is perceived to be seen as protecting your baby, or not doing something that is seen to be protecting their baby. And I so often ask them to sort that out first and then when they come back and they say 'Oh well this is our world view' then I know which way to approach it with them. Which means it isn't just a sentence slotted into a tick list.

The approaches to care dominant in the maternity system also needed to be taken into account. The introduction of an 'evidence based practice' paradigm and the rise in intervention are examples of approaches that midwives needed to manage or incorporate into their practice decisions.

\section{Group 2}

F1

But one of the things that sort of dawned on me this year, what with all the obstetricians stopping doing breeches, which made me really, really angry. Because we start looking at evidence based practice and they are just looking at what the majority were doing. And it sounds like the evidence is showing that for the majority this [caesarean section] is what they're going to go by. And that's it. They've all dropped, every type of vaginal breech birth. But we've got the two outside numbers as well, where it was actually okay for her and she was up in the $90 \%$. Or she was down in the $10 \%$ and they were absolutely satisfied and that was normal for them. And it seems as though we've just excluded everybody that doesn't fit into that middle. And we're just going to go with whatever evidence based practice shows. And we're not going to accept anything that's out of that boundary any more. And that saddened me because in 
one way it's really great. We've been able to prove things with breastfeeding and perineal care. And we've been able to really help ourselves in one way. But it's almost that it's swung back and it's hit us on the back of the head in the other direction. It's kind of like that pendulum isn't it. With all the women going towards the more technological approach, thinking it's safer. And we want it to swing away a bit and actually rely on our skills more. The flip side of that has been women demanding caesarean sections, women demanding induction, women demanding epidurals because that was their choice. And you know I see it affecting everything, the way we measure things.

The differing worldviews of obstetrics needed to be taken into account and seemed particularly difficult to manage. Matching the woman to the obstetrician, while at the same time providing care that matched their own position, was challenging. In mediating worldviews between the woman and the obstetrician, her own sometimes needed to be put aside.

\section{Group 2}

F1

Like I'm very aware when I've got an obstetrician with a $12 \%$ caesarean section rate and then another with an $85 \%$ rate . And when they're so diverse, I know that I'm going to try send everybody to the $12 \% \mathrm{er}$ aren't I? And she gets very, very busy. And there's another one who is very favourable. He had his third baby at home. But he also is not really respectful of midwives or their opinion. He more or less lets you do what you want, but he can make things awkward later. But he's actually quite nice to the women so you're between a rock and a hard place.

\section{Group 2}

F2

I have some very unreliable obstetricians who will fill their heads up with all sorts of rubbish and I have other ones who are so good, who support and still try to stay on the normal track. And there's this huge gap between the two. And I don't feel comfortable because I can't guarantee that we're going to get this one. It could be that one or it might be she's being counselled by this one and seen three or four times in clinic by this one and then on the actual day it turned out to be that one. And I think 'Oh shit!' You know it's just loss of control.

There was some reflection too that the core midwives also expressed differing positions on birth.

\section{Group 6}

\section{F1}

And this midwife went rushing past with IV fluids under each arm. And she said 'You wouldn't believe the post-partum haemorrhages we're having today'. She said 'I think you're ever so brave to be at home with women as they birth. And you don't even use ecbolics! Isn't that scary? 
Because we're using ecbolics and we're still having PPHs'. And I go 'Mmm. Think about it!' (lots of laughter from the group). I know which would be the riskier practice for me. And I'm very happy with feeling safe at home with women. You know it was just such an excellent moment of conversation that's been pivotal to me.

For some midwives there was a discontinuity of worldviews when risk was identified and referrals needed to be made. They felt a dissonance between birth as normal and birth as risky and felt that they needed to shift in their own position when risk became more obvious.

\section{Group 4}

Q

Do midwives manage risk differently from doctors?

F1

Oh I think so, yes we do.

F2

I think so, well I certainly look at everything as normal until I find otherwise. The medical approach is like everything's abnormal until retrospectively things are okay.

\section{Group 3}

F4

I think, certainly there are the majority of the times you are working from the normal model. But I know that there's probably times when I don't work purely in the midwifery model and that's because you do assess that there is a risk and definitely there's other things to think about. And so you are working more from the medical model. For example the diabetic or the twin pregnancy or those high-risk pregnancies. And you do think like that, whether you're doing it consciously or subconsciously.

It was clear then that the midwives managed complex and challenging tasks in a birth environment that was by its very nature uncertain and risky. Nothing in her work seems simple. Birth is set in a context of complex power relations in which the techno rational approach still holds the authoritative position. Midwifery mediates between these positions within the context of attempting holistic and women-centred care. Issues of evidence based care and informed consent are the most current constructions that they have needed to incorporate.

The midwives in the study experienced two particular areas of difficulty as they attempted to provide women-centred holistic care. The first and most fundamental difficulty was that of accountability. They had not achieved a comfortable level of acceptance of the need to be accountable for their actions, perceiving that the 
consequences would be dire. They were fearful and anxious. This then challenged their ability to build trusting relationships both with the woman and with obstetricians. The next area of difficulty was that of authoritative knowledge and the dominance of the techno-rational paradigm. This was an issue of difficulty in itself as it challenged the holistic paradigm. But it also had implications related to accountability. It was seen that accountability would be assessed from the medical or techno rational perspective. It was medicine that the midwives saw as having the authority to decide on what might or might not be considered as appropriate action. Even though many of the midwives were very experienced as autonomous practitioners they still regarded medicine as authoritative, not in the sense of being right but of having the power to claim that they were right.

\section{The findings and risk theory}

This research has shown that New Zealand midwives are both constrained by risk and act in resistance to it. Risk management is clearly an important part of midwifery practice. Midwives are faced with risk on two fronts: risk to the mother and risk to themselves. I have looked at them together as in the real work of midwifery they are so interconnected that to look at them in isolation from each other would oversimplify what is a complex issue. With the support of critical realism I have investigated how midwives deal with risk through two methodological lenses. The extensive approach has been applied using survey and the intensive approach has used focus groups. The following section examines the findings of this research through theoretical lenses. The lenses used reflect the four perspectives presented in Chapter 2. I have not attempted a detailed analysis of each perspective but have chosen to select those parts that are most relevant to the research findings. As with the themes developed in this research the theoretical perspectives are not mutually exclusive. Some of the issues are dealt with in each of the perspectives. The following discussion focuses on the two issues that the midwives found most problematic - accountability and the continued dominance of medicine. The challenge that midwives face in being accountable is a key finding of this research and there are a number of issues relating to it. It can be viewed through the lenses of Beck's 'risk society', Douglas's theories of blame or through the lenses of surveillance and control. And it takes place in the context of the techno-rational paradigm. 


\section{The techno rational paradigm}

The midwives in this research had an uncomfortable relationship with technorationality. Although not asked directly about the use of research, there was little discussion of its use in either the way the midwives assessed or communicated risk to the women. Evidence based practice in relation to risk was not a theme which emerged from the discussion. Instead what dominated was what might be called a 'softer', more relationship based approach, which was focused on protecting the women from any unnecessary anxiety associated with risk, and in attempting to understand what was valued by the woman and her family.

There was further indication of the impact of the techno rational approach found in the midwives' patterns of referral for prolonged pregnancy and lack of progress in labour. From a techno-rational approach these situations are assessed statistically and reflect a numeric understanding of a normal pregnancy and labour. Any women whose progress deviates outside the statistically collated normal curve are defined by medicine as 'at risk', requiring intervention, whether or not there is any co morbidity. Although the focus group discussions have shown that midwives were resistant to this, in that they sought to manipulate the system to avoid referral when women were 'taking too long', it would appear, given the large numbers of midwifery referrals for these reasons, that they are not entirely successful. All the midwives in the groups spoke of the need to deal with the increased risk to themselves when they attempted to do this. Despite the emergence of literature that critiques it, clearly this techno rational approach remains most dominant. It is also interesting that the midwives became anxious when they attempt to provide care that deviated from the techno rational approach. They perceived that there were powerful sanctions and that despite midwifery autonomy and despite their belief in the possibility of normality outside the normal curve, it is the techno-rational approach in the guise of medicine that still holds authoritative knowledge. The midwives became anxious should they be held accountable and perceived that the medico legal environment remains dominated by the medical discourse. It is not entirely surprising therefore to see compliance with this paradigm despite their belief that all was still normal. The midwives' referrals for women who are 'taking too long' and their responses, are important findings of this research. They reflect the continued dominance of the medical approach to birth 
despite over a decade of midwifery autonomy. Despite exhibiting some resistance to this approach midwives continue to comply.

Obstetrics' interpretation of what is normal is related particularly to the absence of pathology; normal is therefore juxtaposed with risk. Increased use of technology has resulted in the discovery of more pathology, encroaching on the normal and increasing the risk. The line between normal and abnormal becomes unclear and contested. Midwives in this study sought to deal with this contested concept of normal in two, almost mutually exclusive ways. They claimed expertise in 'normal' birth (in a physical sense meaning uncomplicated or without obstetric intervention) but at the same time claimed that what might be considered abnormal was so open to interpretation as to be meaningless. In effect, the position that they have taken, that midwives belong with all women no matter how complicated or abnormal their physical care becomes, has prevented them from becoming increasingly marginalized as medicine encroaches into what had previously been part of the 'normal' domain. Whether this has distracted them from focusing on and protecting normal or physiological birth is open to interpretation. It has however enabled them to reclaim a place as the key providers of maternity care in New Zealand. In order to claim this dominant position it would seem that midwives have had to comply with the dominant discourse.

\section{Beck's 'risk society' - lack of trust in the experts}

The dominance of the techno-rational discourse is an integral part of Beck's (1999) theoretical approach to risk. In Beck's terms this approach is part of the development of technology associated with modern life. His contention that we are now in a state of reflexive modernity, resulting in global and personal anxiety about uncontrollability and uncertainty fits very well with several of the findings of this research. In particular they can be interpreted in the light of Beck's commentary about the role of the expert in the 'risk society'.

Beck's theory is based around the ideas of global risk; risk fundamentally constructed by humans in the process of modernity. One might contend that the risks associated with childbirth are 'natural' risks so bear little relevance to this argument. However 
Beck's 'risk society', I would contend, has impacted on the way these 'natural' risks have been perceived and acted upon. Modernity in the guise of medicine's technorational approach has attempted to control these risks and has promised the possibility of 'risk free birth'. Current high levels of technological intervention in birth with no corresponding improvement in perinatal outcome, has given cause to doubt that technology will in fact give us risk-free birth. Ironically the experts can both no longer be trusted to do this yet must be still be held accountable. Midwives are caught in this risk trap. In order to gain their professional status they have needed to comply with the technological project. The way they deal with women who 'take too long' reflects this. Any adverse outcome is interpreted by the woman as a breach of trust, and the expert (the midwife herself) is held accountable. Given the way accountability for adverse outcome has been played out; the name, blame and shame culture this would appear to be the case. Accountability in this research was found to be particularly problematic. But for midwives there is an added dimension to accountability. Not only are they accountable to the woman, but, if they act in resistance to medicine, they will be held accountable to medicine as well. This contradictory and conflicting existence in which they work, both accepting and rejecting the medical or techno rational paradigm, poses significant challenge for the midwife.

They are also subject to the sometimes conflicting constraints of consumerism, in the guise of informed consent, and managerialism, as guidelines or protocols for practice. Annandale (1996) proposed that within a 'risk society' these pose particular problems as the associated anxiety undermines rather than improves the quality of care that is provided. Options for care become increasingly constrained and interventions increase. This research has shown that midwives attempt to manage this dilemma by placing issues of informed consent within the context of connected and trusting relationships with women. They are then able to take a protective role when they need to. The midwife uses her skill and expertise to manage the mother's risk within the individual relationship she forms with her. The effect of managerialism was evident in the way the midwives dealt with the referral guidelines. Many of the midwives considered that the referral guidelines required then to refer more often than was necessary indicating that that there was some compulsion to manage risk in ways that they found problematic. In the discussions with the midwives there was some 
willingness expressed to work outside the guidelines if it was the woman's choice. Again the centrality of the relationship of trust between the two was of central importance.

Whether the centrality of this 'being with women' has developed as a response to the increased individualisation of late modernity or whether it is by its very nature part of midwifery is open to interpretation. What is evident however is that New Zealand midwives have explicitly reconnected with 'being with' in the context of late modernity. In this research it figures more as a resistance to risk rather than as an adjunct to it. Trust, protection and relationship appear as an antithesis to anxiety, blame and individualisation. Although there was some discussion in the focus groups that risk had in effect distanced the midwife from the mother there was still plenty of evidence that attempting to build trusting relationships was still valued and it was this that made risk manageable both in a physical and emotional sense. Connection and trust were important. The midwives in this study demonstrated a commitment to connection by the extent to which they continued to provide care even where increased risk was identified and by the importance that they placed on the quality of collaborative relationships. Where this trust failed, the midwives perceived that risk increased, both risk for the mother and risk for themselves. One could contend that this resistance to risk, by reclaiming the value of connection and relationship, may be a way out of Beck's 'risk trap'.

Using Beck's analysis of the 'risk society', the movement in Western social form from modernity to reflexive modernity has impacted on expert practice by shifting the emphasis from skill to accountability. This accountability for midwifery is expressed in the medico-legal processes. The conversations in the focus groups reflected this shift. Discussions about professional practice were dominated more by the medico legal context in which the midwives lived and worked rather than by concerns related to the acquisition of midwifery skills. There was little discussion about being a better midwife or using scientific evidence and none about meeting the standards of practice or ethics. The acquisition of skills and the provision of care were discussed with much less intensity than was the discussion about their own risk. The balance between skill and accountability therefore seems to have been tipped. The research showed that accountability is reflected as blame and that it is very powerful. The 
midwives were resistant to accountability and were fearful of it, some more so than others and there was a distinct lack of faith in the processes of accountability. It appeared that it was this aspect of risk that was the weak link in the midwives' practice. Although they were interconnected it was the risk of blame which was more problematic for the midwives than risk of adverse outcome for the mother. In a sense the midwives accepted that risk in birth was real and that adverse outcome was sometimes inevitable. They were however anxious about blame.

For Beck anxiety is a central emotion of risk society. There was ample evidence in the findings of the research that midwives go to some effort to attend to the women's anxiety, seeing it as being counterproductive to a good outcome. They seek to normalise, to reassure and to protect. For some of the midwives in the discussions it was evident that in order to do this they would put themselves at increased risk by working outside guidelines for practice. Their own anxiety and their own risk would increase. The extent to which they were increasing risk for the mother when they acted outside the guidelines is debatable and not measurable. Certainly in my experience as an expert witness in the medico legal context I have found that this tendency to normalise the abnormal and to protect the woman from this anxiety does sometimes result in unnecessary adverse outcome in which the mother is left feeling ill informed. Midwives needed to balance what was normal or appropriate anxiety with that which was counterproductive. They spent some time working in the 'grey area' of practice where risk was being perceived and action planned. This balancing act was not easy and many spoke of the disappointment and loss experienced when risk was identified, when they needed to overtly identify risk by making a referral to an obstetrician.

The midwives' attitudes to the risks for the mother were in marked contrast however with the way that they perceived their own risk in the medico-legal context and it is this risk that more closely relates to Beck's 'risk society'. The terms 'the eagle hovering', 'keep on my tracks', 'protecting myself', 'practising defensively', 'it's a killer', cover your arse' 'carrying on my shoulder all the time' reflect a high level of anxiety. Only one of the midwives in the focus groups spoke about the need to put this anxiety in the background lest this 'negative energy' impact on practice. It seemed ironic that the midwives attempted to reduce the anxiety associated with risk 
for the mother, seeing it as counterproductive, yet at the same time did not seem to have the resources to manage or minimise their own anxiety. Midwives saw the woman's anxiety about risk as problematic yet their own anxiety about the risk of accountability was seen as an appropriate response.

\section{Douglas - blaming the 'Other'}

The midwives' understandings of risk and the way they communicated it are reflected in Douglas's (1992) approach, which proposes that risk decisions are more cultural, and value laden than they are rational. This was apparent in the discussions with the midwives in this research. They felt that the relationships they formed enabled them to understand the cultural and social milieu of the women and thus to provide a level of information that they felt was appropriate. Holistic care was valued. This was how they supported the woman to make her risk decisions. The development of the midwife-woman relationship was seen as supporting the midwife to get to know the woman, to understand her values. It would seem that in being in relationship with the woman and in being committed to staying with her, the midwife manages to come to an understanding of the woman's cultural approach and could therefore support and even anticipate the decisions that the woman might make. She may in fact be in a better position to communicate her own knowledge of risk in ways that are more likely to be heard and acted on.

Douglas dismisses the possibility of rational objective decision-making around risk and proposed that it is social rather than scientific knowledge that is the determinant of risk decisions. The extent to which the midwives did not appear to value the probabilistic communication of risk is an indication of the degree to which a social model of birthing is incorporated into midwifery practice despite the dominance of the techno rational approach. From Douglas's perspective it would seem that midwives' approach to risk communication might be enlightened. A closer examination of the ways midwives communicate risk would be worthwhile in order to assess how, if at all, they incorporate scientific evidence.

This cultural approach to risk must also have an impact then on how midwives perceive their own risk and might help to provide some insight as to why midwives are so anxious about it. Douglas's cultural approach to blame provides some insights. 
She describes blaming as primarily a political activity very much related to accountability (Lupton, 1999). For Douglas it is essentially about the maintenance of social cohesion, about keeping things normal. Blameworthiness takes over where the line of normality is crossed. The issue for midwives is: who has the authority to decide where this line is drawn? In terms of blame, midwives viewed themselves as the Other, not only from the women but also from obstetrics. They may then be feeling such a high level of anxiety because they perceive that they are challenging current norms of practice. Midwifery led care certainly has the potential to run counter to a medical-led techno rational discourse. In this sense it is very much a political positioning and they do not trust that the processes of accountability will be fair or just.

However it is not just midwives who are experiencing these high levels of anxiety about the risk of blame and accountability. Medicine itself is, arguably more so than midwifery. It may be related more to the fact that in the globalised society the norms themselves are changing so rapidly that the risk of accountability itself and the sanctions that are imposed are unpredictable.

In order to try and ascertain why there is this high level of anxiety I revisited Douglas's group/grid model to see how midwifery might fit as a subculture in its own right. It was however not particularly helpful. No matter how I played with the data it was difficult to find a fit that could be thought valid in any way. This may have been because midwives are a group of practitioners who are not only dealing with their own risk but also have responsibility for managing others' risk. It seems that it is just too complex. They can be described as both cohesive and highly individual; they are independent autonomous practitioners yet seem to rely heavily on each other both in confirming their approach and in dealing with the 'Other'. They are highly constrained by professional processes that define their scope of practice (e.g. the referral guidelines) and by the medico legal environment (real or apparent) yet they do mange to 'work the system' for the benefit of the women for whom they care. Their positioning of women at the centre of care means that, although they understand the nature of the externally imposed norms they 'work the system' in their attempts to balance the needs and risks of the women for whom they care, and their own risks, 
sitting uncomfortably in the grey area of practice. Their professional identity gives them a basis for claiming this place. Yet it remains insecure.

\section{Surveillance and control}

Although this research has not taken a strong constructivist or post-modern approach it is worth having a glimpse through the post-modern lens to see what it might offer. This position, that risk is more about surveillance and control that about safety, can be seen in the discussions that the midwives had. They are both subject to and the subjects of surveillance. The referral guidelines and the presence of the medico legal processes of accountably can be seen as governmentality at work. The discussion of the findings of this research in light of the previous three theoretical positions has highlighted the importance of control in the face of risk, control not only of the women but also of the practitioners. In a sense this discussion has come full circle, back to assessing the role of the dominant paradigm. Also revealed in the data were the multiple layers of power, both overt and covert. There was power under, over and between. There was collusion and resistance. And underlying this all was the need for control. Risk can then in this sense be seen as a construction, as a way of controlling the masses.

However Castel's (1991) contention, that risk dissolves the notion of the subject and replaces it with risk factors is not found to any significant extent in this research. Although one of the midwives did comment that risk had distanced her from the women compared to how it had been in the past, this research has shown that being with women' is still central and that risk is resisted. Midwives would appear to manage risk by engaging in the relationship rather than by avoiding it. The implications of the growth of surveillance in terms of guidelines and medico legal processes may be an indication that what Castel has noticed, may in fact be occurring. Whether the development of midwifery and its resistance to current formations of risk is an indication of the possibility of a new way of doing professionalism or whether it is an aberration remain to be seen. The extent to which birthing women have embraced knowing their midwife and welcomed the development of the relationships they have with her would be an indication that perhaps we will not get lost in the growing mire of risk factors. This is also supported by the recognition that risk scoring has been replaced, in New Zealand at least, by individual risk assessment. 
The rhetoric at least states that guidelines are there to guide clinical decision making, not to determine it. Humanistic care, albeit fraught, may be winning the day. This may be a sign of the new post-modern order that both Beck and Douglas were hoping for!

\section{Summary}

The findings of this research have revealed what midwifery practice looks like in relation to risk. It has been described and interpreted through a variety of both methodological and theoretical lenses. Midwifery practice exists both because of and in spite of risk. Its focus is fundamentally 'with women'. This connection with the woman is supported by skilled and accountable professional action and a process of 'working the system' in order to meet her needs. This is a complex and challenging task where a growing understanding of uncertain outcome and multiple worldviews must be incorporated. This practice is set in a context of a social and cultural milieu which, although being challenged by the precepts of late modernity, remains dominated by the techno rational paradigm, still embodied by the profession of medicine. This is despite a growing movement towards autonomous midwifery-led maternity care. Midwives resist both this dominance and the insecurities posed by risk by attempting to build connections. These connections are made primarily with the women but are also seen as important between those with whom she must collaborate.

The difficulties that the midwife faces in the provision of care and in the management of risk relate both to the continued dominance of the techno rational paradigm, but also to current societal forms, which reflect increased anxiety about risk. The midwife then is caught in the tensions between, on the one hand, an understanding of the complex and unpredictable nature of her work, and on the other, the still dominant precepts of modernity; controllability and certainty. This tension is manifest in the difficulties that she experiences in accepting accountability and in the degree to which she needs to 'work the system' in ways that protect the woman both from increased anxiety and from unnecessary intervention. 
One of her most difficult challenges is to come to some understanding of the role of normality. New Zealand midwives appear to have taken an approach that reflects a complex and socially mediated understanding of what is normal, rejecting the normal/at risk dichotomy. They have taken a position of being with all women no matter the level of risk. This is their priority, a priority that supersedes the promotion of birth without intervention, which may partly explain their failure to reduce intervention rates. This focus on being and staying with all women has also enabled them to regain and retain the place as the dominant caregivers in childbirth. In order to do this they have also needed to conform to the dominant paradigm, consciously or unconsciously, which may further explain the high intervention rates. One might even go so far as to comment that connection and relationship are valued over the prevention of intervention.

Midwives do however tend to resist current formations of risk. They do this by embracing the tenets of the late modern society. Individualism and consumerism are reworked into making the woman the centre of care. It is made human and manageable by focusing on the quality of the relationship with her. They stay with her even when risk is identified. They are clearly in this sense then not risk averse. They 'work' informed choice about risk in ways that minimise anxiety. They even avoid mentioning the word. There is even some evidence that in their attempts to protect the woman from risk they take on extra risk themselves; they appeared to accept the need to sometimes work outside the guidelines. It is in being a professional that their own risk is managed, risk which is inseparable from the mother's. They deal with 'the eagle hovering' with varied levels of personal anxiety and manage it by being attentive to the whole of their practice.

The following chapter concludes this thesis by drawing on the findings of this research to develop a model of midwifery. This model provides an answer to the question of how midwives in New Zealand make sense of risk in their practice. This model is created through the lenses of the methodological and theoretical tools I have used. It also incorporates the views of other midwives who have researched and written their thoughts through other lenses. And of course I will use my own lens. 


\section{Chapter 9: A Birth Stool for the Midwife - a Model for Practice}

\section{Introduction}

This final chapter draws together the findings and interpretation of the research, into a model for midwifery and develops a theory about the way midwifery is currently practised in the 'risk society'. In this way it takes up the critical realist challenge of abduction and retroduction, using imagination and creativity to recontextualise the phenomenon of interest within a new framework so as to understand what cannot be directly observed (Danermark et al., 1997). The model that I have developed offers both an interpretation of how risk is perceived and managed by midwives and also offers a way forward. Using the model one is also able to identify the parts of practice that are currently most problematic and which need attention. This model is a birth stool, not for the mother this time but for the midwife. Each part of the birth stool is described in light of the developed themes and the implications for practice are highlighted. Other existing theories are used in support of this model. A comprehensive critique of the model is a major recommendation for future research. The chapter uses the model to make recommendations for practice, for policy and for further research, and concludes with reflection on the research process itself.

\section{Model development}

The challenge for me in this final part of the research process was to draw together all the findings into a coherent whole without detracting from the complexity of the process. What I wanted to do was develop a theory or a model of what I had found. Models are exploratory and explanatory tools that can represent relationships. They have several uses: they attract our attention towards specific aspects of practice, they provide a framework for explaining what is happening and they guide our actions by highlighting what is important (Stewart et al., 2003). From a critical realist perspective the development of an explanatory model involves the inferential processes of abduction and retroduction where the data are redescribed and recontextualised (Danermark et al., 1997). What is discovered are 'connections and relations, not directly observable, by which we can understand and explain already 
known occurrences in a novel way' (Danermark et al., 1997). This process does not provide definite truths but can deepen knowledge and provide a basis for further exploration and theory development. The models that derive from these processes are therefore pictures of relationships, so speak to cause. They provide an explanation of what is happening.

For the use of models in science is not motivated by the ability of these models to empirically describe a pure reality. The strength of scientific models lies instead in their ability to break away from a descriptive discourse and provide a possibility to see 'something' as 'something else'. Since the purpose of using models in science is to explore reality by establishing new relations in it the scientific model has a heuristic function in producing new hypotheses and so discovers new dimensions of reality (Uggla, 1994, translated and cited in Danermark et al., 1997, p94).

The model I have produced is my construction of what I saw and interpreted as a representation of midwifery practice in its relationship with risk. It was developed in an iterative process of abduction and retroduction in which the best fit was decided (Byrne, 2002). I see it as a model that may be helpful in the understanding of midwifery practice. It may help in supporting individual practitioners understanding and development of their practice. If the model is found to be robust it could be used by midwifery educators, and policy makers who provide the institutional and funding supports for midwifery. The model incorporates both the quantitative and the qualitative data and also provides a framework in which to interpret theoretical writing on risk.

The model makes an important contribution to midwifery in New Zealand in that it challenges the current discourse of 'partnership'. It does not dismiss it or put it to one side, but provides a structure in which it can be placed, within the context of the real and challenging work of the midwife.

The model is developed from the four key themes uncovered in the focus group data, and from the main findings of the survey. The key theme that ran through the data was that of 'being with women'. 'Being a professional', 'working the system', and 
'working with complexity' were the other three themes identified. It was clear that 'being with women' was intimately connected with each theme. The data then were not mutually exclusive. Complex systems cannot be reduced to constituent parts (Higginbotham, Albrecht, \& Connor, 2001). In the description of the birth stool therefore parts of the themes could have been included in others. Eventually they settled into a place of 'best fit'. On their own any part of this model lacks function and meaning. It must be viewed and used as a whole.

Risk did not emerge as a theme in itself but was part of all the themes. Risk provided the context in which the study was set and was the source of all the data. Storytelling, although not a theme as such, was such an important part of the way the midwives presented their perspectives that I knew it had to be represented somewhere in the model. Throughout this process I was aware that being 'with women' was of central importance. In all the groups the midwives usually discussed issues in terms of their relationships with women and with reference to what the women's needs were. Stories about 'real' women were frequently used to reflect their attitudes. 'Being with women' was also reflected in the quantity of talk that had been coded to this theme.

'Being with women' therefore was positioned as the central theme. 'Being a professional', 'working the system' and 'working with complexity' were themes that were connected to 'being with women'. At this stage of the analysis I had one of those inexplicable brain waves. It was one of those creative, intuitive magic moments of research (May, 1994). Pinned on my wall was an excerpt from one of the participants in which she had used the analogy of a three-legged stool to explain how she understood the concept of normal.

Yeah, I found I had to redefine normal when I started practice. Because I had a concept that you're physically well, that you're emotionally stable and that you're financially or economically stable too. And that was normal. Now what I found in reality is that there's always one leg missing on the stool. There's always a leg missing. And I had healthy strong women with absolutely no money and no partner or he's a crim. It just was awful. And that was normal for her. And then I had really stable relationships and financially okay and everything is hunky dory and then physically, bang, we've got something wrong. So it just seems to me every single client has one leg missing on the stool and that to me is now normal. And that's life. That there are problems and that's normal. So you know that's kind of my picture now is that you're bloody lucky if you get all three at once. 
The stool was a perfect analogy for my study. The model for this study therefore could be constructed as a stool. It is a birth stool for the midwife, a place where she makes sense of risk in practice, a place to sit 'with risk' and 'with women'. The seat of the birth stool is 'being with women'. The legs of the stool provide the other aspects of practice, vital in the provision of care in the current socio cultural context. Being a professional, knowing how to work the system and understanding and working with complexity provide the stability required to 'be' and to 'stay with' women. This model also enabled me to position the role of story telling, something which had been puzzling me. The story telling then became the struts between the legs of the stool, adding stability and security.

Models can be very useful in that they provide a framework for understanding the complexity of the real world and can guide our actions by defining what is important. (Stewart et al., 2003). What became evident during the development of this model was that dealing with risk is no simple matter. It is not just about identifying risk factors and referring, nor about good documentation, nor can it be compartmentalised into one aspect of midwifery practice. What also became apparent is that there is no quick fix for risk. Being a safe practitioner comes from being an effective midwife in every area of practice. The model therefore is a representation of the midwife's work. Its origins come from studying how risk is expressed in practice and it is placed within current understandings of the 'risk society'. Therefore it can also be interpreted as a model for practice as a whole. It is a tool that the midwife can take with her and use wherever she practices. 


\section{The midwife's birth stool: sitting with risk}

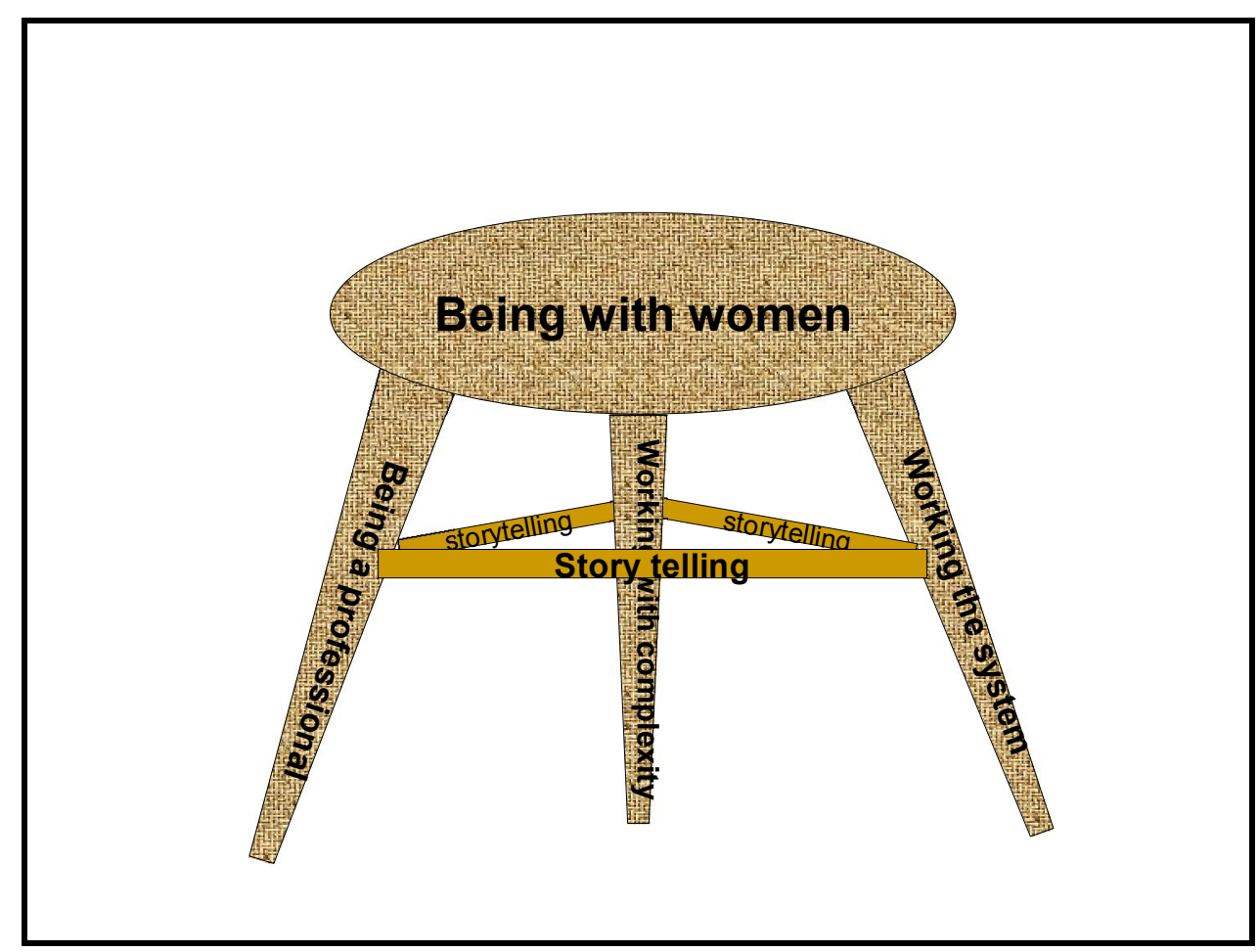

Figure 9.1 The midwife's birth stool: a model for midwifery practice

\section{The seat: being with women}

The most prominent part of a stool is of course the seat. The seat of this stool I have called 'being with women'. It represents the centrality of woman in the provision of midwifery care and the importance of the midwife being alongside her in her journey to motherhood. This research has shown that 'being with women' is of prime importance in New Zealand LMC midwives' care. They stay 'with women' even when risk is identified. Woman centred care is not a new idea. It has been advocated, promoted and described in many reports and by many authors (De Vries, Salvensen, \& Wiegers, 2001; Department of Health, 1993; Kent, 2000; Kirkham, 2000; Page \& Percival, 2000; Shroff, 1997). It is the cornerstone of maternity care in New Zealand (Ministry of Health, 2002).

This research has shown that 'being with' is central and that it occurs in the context of relationship. It is not mere rhetoric. However whether the centrality of 'being with' is located only in the New Zealand context or whether it is more a function of 
continuity of midwifery care needs to be evaluated. Brodie (1996), in her qualitative study of the experiences of Australian team midwives also found that 'being with women' was a core theme. In her study of a small group of Canadian midwives' attitudes to risk, Harding (2000) also found that the midwives' primary focus was on the woman herself, rather than on the 'risk factors' and that the midwives felt that women needed to be cared for holistically.

The extent of the degree to which this focus on 'being with' women is dominant in other midwifery contexts remains to be assessed. I would think for example that the seat of a Dutch homebirth midwife's birth stool might be 'physiological birth' as opposed to the centrality of the relationship. When I visited the Netherlands to talk and share with midwives about how risk is managed they were amazed at how small a New Zealand midwife's caseload was (40-50 per year as opposed to their 120) and how much time New Zealand midwives spend at each antenatal consultation (1hr as opposed to 10 minutes). Spending time 'with women' did not seem a priority. One Dutch midwife suspected that we might be a little co-dependant in our relationships! These contrasts need to be investigated in further research. Midwives who work a shift system in hospitals such as New Zealand's core midwives or midwives in countries such as Ireland, which I also visited, might have 'the demands of the institution' as the seat of their birth stools. However my short visits with midwives in these countries could not be described in any way as comprehensive. Given the high rate of home birth and the low rate of birth intervention in the Netherlands (De Vries, 2004) one might pose the question about valuing 'being with' above protecting normal birth. Whether midwives who work a shift system in hospitals can realistically hold 'being with women' as their core modus operandi remains to be properly assessed. What does need to be highlighted is that 'being with' is set in a particular social and cultural context, unlikely to be repeated identically in other countries. Each context will have its own interpretation of how midwives journey with women.

What is clear however is that 'being with' occurs in the context of a relationship and that relationships matter to midwives. It is within relationships that trust is formed, information is shared, options are offered and decisions are made. Other midwife writers also comment about the importance of relationships in that it is the quality 
both of the relationship and of the communication that are the critical factors in the maintenance of safe and appropriate care (Edwards, 2000; Symon \& Wilson, 2002; Wilkins, 2000). Continuity of carer is important for relationships to be formed. Being with women provides the space and time for the midwife to know the woman, to understand her perspectives and to anticipate her needs. Meaningful relationships are also a key factor in making continuity of carer sustainable for the midwife herself (Sandall, 1997).

A work from a New Zealand midwife that is examining the way midwifery care is provided cannot possibly be complete without some reflection on partnership, the core tenet of New Zealand midwifery (Guilliland \& Pairman, 1995; New Zealand College of Midwives Inc, 1993). Although the term 'partnership' did not figure prominently in the discussions with midwives in this study, it could of course be incorporated into this seat as it is fundamentally about being with women. Central to partnership are the concepts of negotiation, equality, shared responsibility, empowerment, informed choice and consent (Guilliland \& Pairman, 1995). Although the partnership model as expressed in New Zealand maternity care has been well described (Calvert, 2002; Fleming, 2000; Pairman, 2000; Pairman \& Guilliland, 2003; Rooks, 1997; Tully, Daellenbach, \& Guilliland, 1998), the model itself provides little insight as to how midwifery might be undertaken. It is set as an ideal to which to aspire, rather than as a source of support. New Zealand midwives attempt, not without some difficulty to put it into practice. It lacks an explanation of how midwifery might be undertaken either as this partnership relationship is being developed (as it cannot ever be immediate), or of the power imbalances inherent in the midwife-mother relationship (Freeman et al., 2004; Lauchland, 1996), or even in situations where mutuality and shared responsibility will not ever develop (Skinner, 1999). The underlying belief of the principle of the partnership relationship, that pregnancy and birth are normal, healthy activities (Guilliland \& Pairman, 1995) belies the complexity of the real lives of women and of working midwives. Although the model has significant uses as an organisational and political tool and as a practice ideal there is little assistance as to how it might be worked into the everyday messiness of practice. The birth stool model provides this support. 
The term 'partnership' was not prominent in the way the midwives in this study spoke about their relationships with women. What was most prominent was the idea of protection. The midwives attempted to protect women from intervention, from anxiety about risk and from the difficulties encountered when entering the system. In doing this they seemed sometimes to take on added risk themselves. I found this disturbing initially as notions of protecting women could be seen as disempowering and maternalistic. It did not sit comfortably with the notions of empowerment, autonomy and informed choice and certainly not with a negotiated partnership. Help came from the writing of another New Zealand midwife. Smythe (1998), in her study of maternity practitioners and women's understandings of the meaning of safety describes the relationship that the midwife has with the woman as one of concernfulness and that it is within this concernfulness that the midwife can most effectively discern what is safe and what is not. In my research this relationship was manifest as a sense of protection. Concernfulness and protection are close notions. That two pieces of significant New Zealand midwifery research should discover this as an integral part of the relationship is worthy of careful consideration. It is challenging to sit concernfulness and protection alongside negotiated, equal, fully informed and autonomous decision-making. Perhaps protection has more to do with trusting, connected human relationships and therefore with love.

Being with women then is a central and critical aspect of midwifery practice. It is necessary yet not sufficient. On its own it does not help in understanding the complex and challenging work of midwifery. On its own it is a seat without legs. It doesn't lift the midwife off the ground. The legs of the stool provide the other aspects of practice that are vital in the provision of care in the current socio cultural context. Being a professional, knowing how to work the system and understanding and working with complexity provide the stability required to stay 'with women'. It is the legs of the midwife's birth stool that give the midwife support. The legs help to lift the midwife above the 'messy swamp' of practice (Schon, 1983). 


\section{The legs of the birth stool}

\section{Being a professional}

This study highlights the important part that being professional plays in the lives of midwives. Professionalism has been criticised for being elitist, exclusive, sexist, racist and classist (Thompson, 2002) and the professionalisation of midwifery is not always thought to be a worthwhile project (Lay, 2000). However this study has found that New Zealand midwives 'do professionalism' differently. It is embedded in and dependent on the relationship that they have with women. This was also described by Tully (1999) in her study of midwifery autonomy in New Zealand. She described it as a 'new professionalism', relational and connected. This theme is not uncommon in writing on professionalism within maternity care (Page et al., 1999,Stewart, 2003; Thomas, 2000; Wilkins, 2000). Kirkham (1996) proposes being a professional involves autonomy and self regulation and that with professionalism comes ownership of knowledge and power relations. She says that professions inevitably seek to control and define the reality that the client experiences and that both being with the woman and being a professional create tensions. This new professionalism has changed the way that the professional deals with risk. The expert who 'knows all' is now a double risk manager, managing not only the risk to the woman but also their own risk. They must then decide whose risk takes priority, their own or the consumer's. This 'new' professionalism was present in this study. There were two aspects of being a professional that were important. The first was being skilled and the second was being accountable.

\section{Being skilled}

Having the skills needed to assess risk was important and the midwives were comfortable that they had the skills required to do so. Many of the midwives said that continued involvement in care into the secondary maternity context meant that it both maintained their skill base and stimulated learning. This was seen as important in order to be able to assess risk and to be able to provide accurate information for women. What they were less skilled in was in assessing and communicating the techno rational evidence, and then taking it into practice. They seemed uncomfortable with the 'ecological fallacy' and were unsure of how to deal with it. The issue of 
whose knowledge could be considered authoritative further complicated this. Stapleton, Kirkham and Thomas (2002) in their qualitative study of how evidence based leaflets were used in maternity care confirmed this finding. They found that although health professionals were positive about the leaflets, hierarchical power structures meant that obstetricians defined the norms of practice and therefore what choices were available. Choices about how to use the evidence therefore were made on the basis of informed compliance rather than informed choice. In my study the provision of information was further complicated by the midwives' need to protect the woman from fear and by the need for accountability.

\section{Being accountable}

Current constructions of professional practice are very attentive to accountability and are fundamentally related to the centrality of the consumer. Being a professional means being accountable. As discussed earlier, the current state of the 'risk society' means that professionals can be blamed when an adverse outcome has eventuated. It is here that we see the midwife as being 'at risk'. She is at risk of litigation. The difficulties the midwives expressed with personal accountability for practice was a key finding of this research and is, I think, the weakest part of the birth stool model. It detracts both from the midwives being able to use their professional skills in protecting normal birth and from trusting relationships with women. The midwives expressed much more comfort about dealing with the women's risk than in dealing with their own. What I found was that although the midwives attempted to put risk into perspective for women in order to minimise fear, they were unable to put their own risk into perspective. Just as the general public are becoming increasingly anxious about very small probabilities of risk, so to are the midwives themselves. Their own fear was palpable and it was the fear of accountability for poor outcome rather than of the outcome itself that was the cause of their fear. In Chapter $1 \mathrm{I}$ described the incidence of medico legal activity in New Zealand, which is really very small. That it should have such a profound effect on how midwives feel about practice is revealing. Getting accountability into perspective is one of the most important challenges midwives currently face. The risk of being held accountable within the medico legal processes are very small and must be kept in perspective lest practice be paralysed. 
Accountability is an important aspect in midwifery and can be seen as a valued part of practice. It does not necessarily mean always accepting responsibility for any adverse outcome but it does mean that the midwife must be accountable for her decisions and her actions. This accountability is primarily to the woman, but also to the profession and to the public. Accountability is a public right and it must be acknowledged (Leape \& Berwick, 2002). Accountability is a valuable tool for the midwife and she should not be afraid of it. Accountability facilitates an honest, reflective relationship. It helps to keep the midwife attentive and motivates her to keep her knowledge and skills maintained. It is a powerful motivator in keeping the woman at the centre of practice. However it does sometimes mean that the midwife must be accountable to the public and to the profession within the medico-legal context and it is this aspect of practice that is most feared.

Midwives are not alone in expressing anxiety related to accountability. Many professionals are finding this problematic given the prominence of risk in today's society. Reflecting on the birth stool in light of the data shows that difficulties with accountability has weakened the 'being professional'leg. Accountability has shifted the power relations. The midwife is accountable on two fronts, to the woman and to the system dominated by medicine and the techno rational paradigm. Safety for the midwife in this context includes attending to the whole of practice, to all the parts of this midwife's birth stool. It remains based in a sound relationship with the woman, in providing skilled care, and also in an understanding of and connection with the systems that are in place. It is supported by an understanding of, and negotiating between, the complex and often-contradictory environment in which she works.

\section{Working the system}

The next leg of the stool I have called 'working the system' and is an important part of providing care, care that continues to be based on staying connected with the woman and her experience. I have called it 'working the system' as it is a reminder that the focus remains on being 'with women'. Midwives work the system in order to meet the needs of the woman. This leg reminds us that we do not provide care in isolation. We provide care within a system, and it is important not only to understand how the system works but also to participate in it. There were three aspects of working the system revealed in this study. They were: managing the relationships, 
using the referral guidelines, and the role of power and authoritative knowledge. They could be described as the 3 Ps: people, processes and power. These three are, as all aspects of this model, deeply interrelated, but I discuss them as separate entities here for the purposes of clarity.

\section{People}

The first and possibly the most crucial, is the development and maintenance of successful collaborative relationships with others in the system. Within my research, not only was the quality of the relationship with the women important but the quality of the relationships with obstetricians and other midwives was also seen as crucial. The midwives felt that both they and the women were much safer where respectful, trusting relationships had been developed with those with whom they needed to collaborate. These relationships cannot be assumed but must be actively sought and maintained. They also include relationships with work partners, their midwifery peers. It includes those who provide secondary maternity services, the obstetricians and midwives in the hospital. For the midwives in the study it was the quality of relationships with others in the system that mattered. Personal knowing was important and trust and respect had to be earned and had to work both ways. Seventy two percent of the midwives agreed to some extent that obstetricians in their area supported continuity of midwifery care and $64 \%$ agreed to some extent that there was excellent collaboration between primary and secondary care. Where relationships were successful the midwives themselves felt safer. Close working relationships are essential (Platt, Angelini, Paul, \& Quilligan, 1985; Wiegers, van der Zee, \& Keirse, 1998).

It was satisfying to discover that despite widespread belief in the community that relationships between doctors and midwives are poor this study illustrated that in general they were satisfactory. However $14 \%$ of the midwives did not think that obstetricians in their regions supported continuity of midwifery care and $24 \%$ disagreed that there was excellent collaboration. Work needs to be done to improve these proportions. Wallace et al (1995) also found high levels of satisfaction with interprofessional relationships in what was thought to be the first study focusing on the quality and quantity of interaction between medical and midwifery staff in a hospital environment. One might ponder whether poor relationships are more rhetoric 
than reality and question whose needs are being met in continuing this discourse. Smythe (2000) also found that relationships with co-workers were important. She commented that trusting, collaborative relationships facilitated the sharing of concern and that trust is developed within the relationship. One cannot assume or presume trust either with the woman or with other professionals. It is not automatic and must be earned. In order to do this personal knowing is important. Certainly there is a great deal of emphasis placed on teamwork and collaboration, but little effort put into developing it. If you were to compare the energy and expense that goes into developing guidelines and protocols for practice and compare this with attempts at connecting practitioners together as collaborative teams, you would find that the guidelines take a much larger proportion of time and energy.

\section{Processes}

The second aspect of 'working the system' relates to understanding and working with the processes that the system puts in place. In this research these processes were identified as the referral guidelines. The guidelines provide a framework for the midwife to use in decision-making related to complex or 'at risk' pregnancies. The referral guidelines are not essentially about risk screening but are about delineating the boundaries of professional practice and facilitating inter-professional collaboration. The guidelines are an indication of the scope of practice of a midwife and can be used as a guide to indicate the appropriate level of care. This was reflected within this study in the finding that although some midwives did find that the guidelines restricted their practice, most found that they were a useful tool in indicating when to seek obstetric assistance. There was also a statistically significant relationship between the midwives' positive attitudes to the referral guidelines, and whether or not they worked in a supportive and collaborative environment. Common understandings seemed important.

Guidelines are not always appropriate in individual circumstances however and there will always be some women who do not want to follow them. Sixty-four percent of the midwives in a study of midwives and obstetricians in the United Kingdom, said that they had cared for women who did not want to follow guidelines for practice (Symon, 1998). Similarly in the current study midwives also discussed this as a frequent and normal part of practice. Risk may or may not be increased when this 
happens. What is important though, is that the midwife understands that this is happening so she can assess whether measures need to be put in place for added protection both for the woman and for herself. This may be as simple as careful documentation.

For the purposes of the model the idea of guidelines can be extended to include all the requirements that the system sets in place, such as protocols, funding and contractual arrangements, professional standards and the required competencies. Working the system also involves participating in organisational risk management and clinical governance processes (Blandford \& Smyth, 2002; Cooper, 2000; Wilson, 2002). It is important not only to know what the organisational expectations are but also to be involved in developing them and therefore in owning them. It was noticeable in this research that the midwives often distanced themselves from the system. For example the referral guidelines were talked about as though they were imposed by medicine which is interesting given that their own professional body was an active participant in their development. Midwives seemed to take the position of powerlessness even when they had power.

\section{Power}

There is an enormous body of literature examining power relations within maternity care all of which make reference to the dominance of the medical paradigm and the position of obstetrics (Arms, 1975; Benoit, 1997; Brownlee, Mcintosh, Wallace, \& Murphy-Black, 1996; Kirkham, 1996; Kitzinger, Green, \& Coupland, 1990; Mander, 2002; Rich, 1976; Rooks, 1997; Walker, 1976; Witz, 1992). This is also confirmed in the theoretical perspectives on risk presented in Chapter 2, which discuss the dominance of the techno rational paradigm and its association with medicine. It is of no surprise therefore to see this issue emerge as part of this study. It would be naïve to talk about the midwife's relationship with the system without acknowledging the existence of power relationships and the need to manage power disparities. There is considerable play of power in the ability to define who and what is risky and what should matter (De Vries, 1996; Stapleton, 1997). For the midwife the power of the institution and the power of obstetrics are real and challenging (Freeman et al., 2004). There exists a hierarchy of authoritative knowledge within maternity care in which the 
technical expertise of medicine is dominant (Jordan, 1997). This process comes to be perceived as natural and legitimate:

Authoritative knowledge is persuasive because it seems natural, reasonable, and consensually constructed. For the same reason it also carries the possibility of powerful sanctions, ranging from exclusions from the social group to physical coerciveness. ... The power of authoritative knowledge is not that it is correct but that it counts (Jordan, 1997, p58).

The risk discourse as it is currently constructed, principally by medicine, can be viewed and used as a tool of control and surveillance both of midwives and mothers. Power can be hidden within this risk discourse. It is not always overt but can be open to challenge. The midwife needs to know however that confronting this medical power is in itself a risky strategy and must be approached with caution (Fahy, 2002). In this study it was disconcerting to see how vulnerable midwives felt. This was despite the fact that in every medico legal forum in New Zealand where midwifery practice is examined, midwifery expertise is provided and acknowledged. Although far from complete, we have made important inroads in the establishment of midwifery knowledge as authoritative. It would seem time for practising midwives to acknowledge this.

The midwife is not only a subject of power in terms of obstetrics but can act as an agent of power herself. The discourse of equality central to the notion of partnership can obscure the power relations inherent within the midwife-mother relationship (Freeman et al., 2004; Leap, 2000; Skinner, 1999; Smythe, 2000). In research that examined how power is expressed within the midwife-mother relationship Freeman (2004) asked where expertise, experience, knowledge and legal requirements fit into a partnership model of care. Their research found that in $85 \%$ of cases of decisionmaking that involved high-risk situations, it was the midwife who primarily made the decision. As a response to this they developed a model of decision making in which, as risk increased, so too did the dominance of the decision-making by the midwife. The midwife then needs to understand the power relations that exist and work to claim her own power in the context both of providing care and protecting and enhancing the power of women. She needs to be mindful of the rhetoric of equality both within what is called 'collaborative relationships' with doctors and 'partnership 
relationships' with women. Working the system then also means working the power relations. There are power relations between midwives and obstetricians, between the midwife and the woman, and between the midwives themselves. There is power 'over', 'between' and 'under'. It is important for the midwife to be aware of these and manipulate them first and foremost in the interests of women.

In order to manage risk and safety then, the midwife must learn to 'work the system'. She should keep connected and keep involved, actively participating in building collaborative relationships and in the planning of guidelines and policy. Safe practice is connected practice. Midwives need to build solid and collaborative relationships and they need to be involved in policy making and in research. They need to be active members of their professional organisation and develop a system for their own practice that is safe and sustainable. They need to both claim and share their power, and challenge power systems that are unjust. All this is challenging and complex.

\section{Working with complexity}

Complexity is such an integral part of midwifery care that it deserves a leg of the birth stool all of its own. In being alongside women things are often complex and unclear. The midwives in my study, in reflecting about how they managed risk, frequently talked about working in the 'grey areas', not only in decision making around the physical processes of birthing but also in the relationships that they developed with women, with medicine and with the system. There was a good deal of unknowing and uncertainty. The more experienced midwives described how these grey areas of practice became even bigger the more experienced they became. Yet as they became more experienced uncertainty and complexity became less disturbing as their practice wisdom grew. They accepted that risk and uncertainty were normal parts of practice. They accepted the need to understand and accommodate many different perspectives reflecting the differing underlying cultural and value systems in which both they and the women lived.

Midwifery practice is not simple but we seldom acknowledge it in ways that are overt. Complexity is an expected part of practice and needs to be acknowledged and supported. Even the idea of normal is complex. We need the skills to accept and 
work with uncertainty in a world still dominated by the need for control and predictability. Working with uncertainly and complexity is often our unseen work. This model makes it explicit so we can deal with it, learn from it and teach it. We need to be open, flexible and alert. We also need the skills to mediate between different worldviews. And we need to be aware of our own worldview and how this impacts on our practice. The dilemma for midwives in this study is that in being 'with women' even when risk is identified we get caught by the technology and intervention itself becomes normal. Somehow we need to get some clarity of purpose and focus.

Midwifery practice is not simple. Developing relationships with women is not simple; being a professional is not simple; maintaining collaborative relationships is not simple; managing risk is not simple. As Heyman (1998) states,

Difficult decisions about risks entail weighing-up and trading-off qualitatively different values, for example autonomy versus safety, or quality of life against longevity. Such trade-offs of finely balanced but incompatible ends require value judgements which individuals and social groups make differently, and which cannot be meaningfully aggregated or anticipated. The persons most directly concerned in a health decision may have difficulty in deciding or even predicting what they would do. Health professionals who wish to help clients to manage risk need to grasp the complexities involved in such reasoning (p9).

Dealing with risk then is complex and challenging work, done in the context of the primacy of 'being with women' and alongside astute, professional care. If it is so complex then, how might the midwife go about it? Understanding some of the theory about how complex systems work might be of assistance. Complexity theory tells us that tension and paradox are natural phenomena. Problems are often not resolvable through simple cause and effect processes. Instead they occur in ways that are nonlinear. Unpredictability is inherent in complex systems although patterns emerge through inherent self-organisation. There always remain things that are unknowable (Plsek \& Greenhalgh, 2001). Paradox and uncertainty however are not necessarily negative. They can be used as sources of change and improvement. Complexity theory rejects the machine model of the human body and of health systems, where the 
whole is broken down into smaller and smaller parts for treatment or intervention. Being with women then requires a holistic approach, accepting unpredictability yet building on the emergent forces and processes that become evident. It requires careful observation and the application of astute, involved, creative and intuitive care.

Midwives also value difference but there is complexity in managing this. It involves working with complexity, paradox and contradiction. Managing different worldviews is not just a matter of the medicine versus the midwifery view. This oversimplifies the problem. We have to manage the varied worldviews of the women we care for and of other midwives we work alongside. The birth stool provides a model of how we go about doing this. The midwife becomes a mediator between the woman's risk framework, her cultural position and that of the dominant value system, the technorational approach. She too has to place her own risk framework into the equation and to assess whether she considers it right or wrong.

The birth stool provides a framework for dealing with risk and safety, complex concepts embedded within and between complex systems. It provides a holistic approach and challenges the midwife to attend to each aspect of her practice in ways that acknowledge this. It is clear that there is no one truth about what is risky and what is safe but one that is viewed through multiple lenses. It is the task of the midwife to understand these multiple lenses. To some extent then she can be seen as a 'paradigm broker', mediating between different ways of knowing and understanding (Skinner, 1999). The midwife deals with risk between the woman and the obstetrician. She mediates the reality of the woman's understanding and the obstetrician's understanding knowing that the authoritative knowledge stands with obstetrics. She does this by sitting on her birth stool. This stool is situated in a risk framework of accountability, again dominated by obstetrics. Midwifery situates itself both alongside medicine in its surveillance of women but also alongside women. It is therefore in a 'between' position. Staying both with women and with risk, positions midwifery in New Zealand in a way which challenges the traditional definitions of normal and has the potential to work creatively in mediating realities. Midwives' role as paradigm broker may be central for it to be able to work creatively. This would reflect a new age, even new post-modern approach. 
Davis-Floyd (2002) describes the postmodern midwife:

By 'postmodern' midwife I specifically do not mean midwives who uncritically accept either their own ethno-obstetric system or that of biomedicine, but rather midwives who fully understand these in a relative way, as different ways of knowing about birth, discrepant systems that often conflict but can be complementary. Ideally, the post modern midwife knows the limitations and strengths of the biomedical system and of her own, and moves fluidly between them to serve the women she attends. She plays with the paradigms, working to ensure that the uniquely woman-centred dimensions of midwifery are not subsumed by biomedicine. She is a shape-shifter - she knows how to subvert the medical system while appearing to comply with it, a bridge-builder, making alliances with biomedicine where possible, and a networker (p2).

Attempting to be a postmodern, paradigm broker can also mean that the midwife might get caught in the middle. The referral practices and attitudes of midwives in this study reflect that this may be happening. Their practices and attitudes towards referral for prolonged pregnancy and prolonged labour reflect that they both incorporate the risk discourse into their practice yet at the same time are uncomfortable with it. Their commitment to being with women is clear. Their willingness to share responsibility is clear, yet also clear is some complicity in current dominant risk management. Yet they do manage to stay 'with women' despite it all. They deal with the complexities of working the system and with current discourses of being a professional and manage to be both agents of medicine and agents of the women for whom they are caring. It is held together by the human relationships which keep them sane and keep them focused.

\section{The struts: Storytelling}

The last parts of the birth stool are the struts. Struts connect the legs with each other, support the seat, and help to hold the stool together firmly. I have called the struts of this midwife's birth stool 'story-telling'. In every focus group storytelling was abundant. So much so that it sometimes appeared that the midwives were always answering my questions with a story and swapping stories with each other. Initially I 
found this frustrating. I had wanted to get their opinions and to hear what they thought rather than to have another story. However I eventually realised that the stories themselves revealed their opinions and also played an important part in sensemaking not only for me and for each other, but also for the midwives themselves. It was important to pay attention to them. Once I became comfortable with the stories I realised how valuable they were and how pleased I was that I had chosen the focus group approach. There was such resonance in the stories which I am sure helped the midwives to engage more fruitfully with the issue and in the conversations with each other.

Analysis of the stories then contributed to the coded data. However there was more to the storytelling than this. Storytelling in itself seemed to be an important process for the midwives on a number of levels. Telling stories seemed to keep the midwives connected to the real experience of the women. Each story revealed the complex and contradictory nature of midwifery as a professional activity, always drawn back to the connection with the real lives of the women for whom they cared. They used stories to illustrate their role as professionals and to show how the system they worked in functioned. They used them to explain and justify their opinions and their actions. Stories held the whole together. They were like the glue that kept the connections between the varied aspects of midwifery practice firmly in place. It was important to include storytelling in the model not just as coded themes but also as a process in their own right and I pondered for some time how to do this. The struts seemed the perfect place for them, not absolutely critical to practice itself but important in helping to keep practice integrated and connected.

Midwives are great storytellers. We tell stories to share and connect with each other. The stories can be both healing and sense-making. Stories express and create the norms of practice. Sharing stories keeps us connected with each other, helps us to understand the complexities of practice and provides a way of understanding what is expected. Storytelling keeps us mindful and reflective. Stories can both challenge and affirm us. They bring to our attention that which we may have let go, and offer us other possibilities for action. They help to keep our practice connected and safe. How we construct and reconstruct reality tells a lot about who we are and what we 
value. We tell the stories to each other, to connect collegially, to have a good laugh or a good weep. We use them to seek support, to challenge and to justify.

Story-telling is an important learning tool and has been increasingly advocated in the teaching of health practitioners (Fraser \& Greenhalgh, 2001; McDrury \& Alterio, 2002; Stewart et al., 2003). Kirkham (1997) tells us that stories give coherence and meaning to our experiences, and that they demonstrate and consolidate our value systems. Stories may have multiple meanings with many layers of social constructs embodied in them. They convey who we are and how we place ourselves in the world (Linde, 1993).

The notion of storytelling can be extended to other areas not usually included in how stories are commonly understood. We tell a story in clinical documentation and in formal practice review. We tell stories to women we care for and sometimes we have to tell the story to disciplinary bodies and to our professional organisation. Story telling is probably the most valuable tool in the teaching and development of midwifery as it is in story telling that the messy and real complexity of practice is revealed and understood. We use stories to rationalise and justify practice and to keep our ideas connected to real practice and real women. Stories are not always a positive thing. They may help us to believe our own rhetoric, recreate an experience into something it wasn't, relate confidential information inappropriately or reflect bullying or abusive behaviour. We can embellish some bits, stress others and choose to leave some bits of the story out. Stories need to be attended to carefully and thoughtfully. Storytelling then is the last piece of the midwives' birth stool, helping to give it security and solidity, holding it firmly in place. In one of the focus groups I asked the midwives why they told so many stories. They just laughed at me and said 'Well that's just what midwives are'!

\section{Sitting on the birth stool: Taking the model into practice}

Midwives can take this birth stool into practice. Being an effective practitioner is about sitting on the birth stool; being connected with up to date knowledge and skills; being connected to the complex systems and processes that guide practice; being connected to co-workers; and above all being connected with women. 
Birth stools have a long history and are seen as one of the midwives' ancient tools. Their purpose has been to support the natural process of birthing. Originally their structure was simple. The midwife would carry her birth stool with her and the mother would use it as support as she delivered her baby, always upright. Over the period of medicalisation of birth the stool became transformed. Technology and supine birth transformed it into the modern delivery bed. Originally the stool reflected community concerns and the role of the midwife as an important part of the community. The midwife was respected and birth was seen as a natural process where intervention was not practiced unless death was close. As doctors took over control, birth became a pathological process associated with illness and abnormality. The return of the birth stool in more recent times represents a resistance to this approach and an attempt to return to the age of midwifery where birth is viewed as a women-centred, natural event (Banks, 1999). It seems a good time for the midwife to have a stool for her own use.

This midwife's stool has three legs and there are a number of characteristics of threelegged stools that make them unique. It makes them sturdy and stable. Provided they are reasonably in proportion, the legs do not have to be exactly the same length or shape. They are resistant to wobble. As long as they are well glued, they are secure and long lasting. They can be made of different sizes and materials but will still be recognisable and functional. They are simple yet can be embellished and decorated. They can be rough and unfinished or smooth and polished. They are ancient yet are still used today. The use of a stool as a model has the potential to speak to midwives.

Every midwife is different. We bring our own personality and our own story with us wherever we go. We have our own strengths and weaknesses. Some of us will have special skills in some areas and will need to pay particular attention to others. But all parts of practice need to be balanced and integrated. We need to attend to women first and foremost but we also need to ensure that our professional practice, our active participation in the maternity system and our ability to work with complexity are skilled and effective. We need to tell the story with integrity and thoughtfulness. The realities of midwifery in the real world mean that we seldom if ever have a perfect stool. For example, where the relationship with the woman is challenging and 
difficult, strong well-connected legs will support it. Where the new practitioner is still developing her skills and expertise, her relationship with the woman and the connections and involvement she develops with the system will ensure that she is supported. Where deficiencies in the system challenge the ability of the midwife in her work, an astute midwife who understands the power dynamics and the variety of apparent worldviews, and who is skilled and accountable in the context of a strong relationship with women can still provide effective care.

This birth stool is easily transportable and sturdy. The midwife can take it with her wherever she practices. She does however have to sit on the whole stool and be attentive to all its parts. When any of the parts give way it will not provide support. It is also sometimes important to get off it to give it a polish or even just to give her legs a stretch (doing some more study or just having a holiday). It is also useful to have others looking on to warn of cracks appearing or to ensure that it is placed in the right position (professional standards or practice review). If any of the legs get too out of kilter or the glue comes unstuck the midwife will fall off her seat; being with women will not be an option. Some of the midwives in my research were about to get off their stool. For one, being with women had become no longer tolerable as she found the demands too overwhelming. She was unable to work the system to meet her own needs. For another the pressure of the medico legal environment had spoiled practice and she wished to be free of it. She no longer had trust in either her medical colleagues or in women for whom she cared. She herself felt unsafe. The demands of being a professional had become overwhelming and isolating. Another was a very new midwife who had found that 'working the system' as a new practitioner was just too complex and too scary. She lacked the support of others around her. Yet for most of the midwives in the study there was a great deal of satisfaction, sustained by the quality of relationships that they had with women giving birth. They were confident and safe practitioners, challenged but not overwhelmed by the complexity of their work.

\section{The birth stool: What needs attention?}

This study provides midwives with a model for practice. It has done this by looking at how midwives manage to make sense of risk. Risk has provided a way of 
examining what midwives do and how they do it; it has provided a door through which the whole of practice might be examined. It has therefore provided a broad view and of course has opened up many areas ripe for further research. Each reader will have their own questions as they come with their own perspectives and ideas. There are many that appeared for me along the way. Some of the issues that have emerged for me as I interpreted the results and reviewed the related literature include: the impact of moving uncomplicated childbirth out of secondary and tertiary hospitals; the nature of collaboration and how to improve it; how to improve the care for women who 'take too long' having their babies; more detail about the implications of referral guidelines; the impacts of caseload and experience on care; more appropriate funding when care becomes complex; the implications of over identifying risk and ways to avoid it; the nature of storytelling and reflection and its real impact on practice. All these issues can be located somewhere on the birth stool and are of interest to practising midwives, policy makers and educators.

There are three areas of concern however, which need special attention, one on each leg of the birth stool. They have been identified as the model was described but I mention them again briefly here in order to emphasise their importance. The first is accountability. Accountability needs to be reconfigured in the lives of midwives. The risk to us is small and we seem to have the fear of this quite out of proportion. A shift in mindset is needed. Accountability is a powerful and positive tool in ensuring that women get excellent care. Where we fail to do this we must be able to acknowledge it. The processes of accountability within New Zealand are of course not perfect but we need to acknowledge and trust the expert midwives who are involved in reviewing practice. We must remember that we are all capable of making mistakes and that being accountable is hard. We need to support midwives who are being held accountable and challenge those who are not. We now have our own regulatory body, the Midwifery Council of New Zealand. We have control of how we do this and we need to ensure that the processes are transparent and just.

The second area of concern is about power and how we perceive it and use it. There has been so much emphasis placed on the power that obstetrics holds over midwifery that we do not understand that this power is neither omnipotent nor eternal. We are failing to notice that this is currently under challenge. In Beck's 'risk society' he 
proposes that current formations of risk challenge the techno rational paradigm and that a new social order needs to be created. Douglas too proposes an alternative position to blame and sees current constructions of risk as being under threat. Critical realism and complexity theory too have a view of the changing nature of society and the way power is manifest and might be constructed in the future. What we do know in midwifery is that we have considerable power and need to be able to use it. We are the main caregivers in birth; we have autonomy of practice, fully supported by the institutions that regulate us and are now well ingrained in New Zealand society. We need to claim this power and enact it, not as obstetrics has but in more inclusive, connected ways. We need to use it effectively and to share it appropriately.

The final area of concern relates to the complexity of practice. This study has highlighted the complex nature of the work we do and proposes that we need to understand it and embrace it. We need to acknowledge and develop our skills as paradigm brokers; mediating between the multiple realities of the women we care for, the practitioners we work alongside and the world in which we live. We need to teach it to our new midwives and develop it in each other. We need to be flexible, attentive and creative. Only then will we free ourselves of reductionist assumptions and restrictive practice.

What does not need particular attention is the seat of the birth stool. Midwives in New Zealand have demonstrated an extraordinary commitment to being with women. We stay with them right through the childbearing process. We enjoy the relationships we have with them and show concernful and protective care. What we do need now is to work on the legs so that this care can be maintained and enhanced. In this way the love that we express in terms of our connection with the women will not be undermined by the fear that we feel. Maybe then we will free ourselves from the 'risk trap' to support birth in ways that reduce intervention.

\section{Conclusion}

This thesis asked how midwives make sense of risk in practice, both in what they do and how they feel and I chose the referral for obstetric consultation as a place to investigate this question as it is a site where risk is expressed in practice. I have 
looked at risk from a variety of theoretical perspectives; the techno rational approach and its impact on normality and probability; Beck's proposal that we live in a late modern 'risk society'; Douglas's cultural approach to risk and blame; and a brief look at the post modern approaches of governmentality and surveillance. It was apparent that midwives' understanding and management of risk could be interpreted from all these positions. The midwife must deal with the mathematical calculations of risk but at the same time is deeply immersed in the socio cultural environment in which she practises.

The decision to take a critical realist stance in approaching the research question acknowledged the fact that midwives in practice must deal with risk at a variety of levels. Critical realism proposes multi-layered reality. Research taking this stance seeks to explore these layers in order to uncover the generative powers, thus speaking to causation. What is revealed is new knowledge which, although always fallible, seeks emancipation. I explored the referral for obstetric consultation from both an extensive and intensive position; a national survey of the referral practices of LMC midwives, followed by a series of focus groups with midwives in a variety of New Zealand locations.

The findings of this research have been presented on three levels, each level building on the findings and interpretations of the previous level. This followed Bhaskars' description of a multi-layered and stratified reality. I first presented the extensive or quantitative data and discussed the findings. The focus group findings were then interpreted incorporating the survey findings. Four themes were identified: 'being with women' 'being a professional', 'working the system' and 'working with complexity'. These were further interpreted in light of existing risk theory. The next level of analysis led to the development of a model; the birth stool for the midwife.

So what was discovered? That midwifery is both constrained by risk and acts in resistance to it. That in managing risk, being with women is still most important to midwives and that relationships are central. Being 'with women' is midwives' business. Being with women is supported by being a skilled and accountable professional, who works the system and understands and can work across multiple world views. Midwifery then presents itself as a different way of relating to risk, of 
being professional and of using knowledge. Midwifery knowledge in this context is synthesised from across many disciplines. This makes it open to new ways of dealing with the world in all its complexity. One might call it a 'transdiscipline', ready to move into what ever 'postmodern' might become. Perhaps it is the embodiment of what Bhaskar calls a new science:

Bhaskar sometimes talks of an 'occasional' role for the philosopher as the 'midwife' of new sciences.... I take it that a good deal of the motivation for his work is to replace this positivist brood by something both more scientific and more conducive to human emancipation. At times he seems to suggest that these better human sciences are already overdue for birth, and merely await the arrival of a suitably qualified midwife (Collier, 1994, p20).

Bhaskar is unlikely to have anticipated that a 'real' midwife would use his work and I do not lay a claim to being 'suitably qualified"! However the science and art of midwifery, its ontology and the way it sources knowledge could be seen as a new way of viewing the world opening up possibilities for engaged, holistic and connected knowing. The birth stool is an example of this. It is a place for her to sit and 'be with women'. We are not shackled with any particular 'ology' and so are free to explore many ways of viewing the world. This is what we do.

As with the birth stools of old, how this one is constructed reflects how society is constituted at the moment. This birth stool represents the way today's New Zealand midwife 'sits with risk'. The stool is my analysis and my modelling and is therefore my offering; it is one way of understanding, one view into the issue. I have made my place clear. The process of the data gathering and data analysis are transparent. It is therefore up to the reader to read, think, critique, and play with it differently if they will. I have started with a very simple model but the possibilities for redesign are limitless. Individual midwives might like to construct their own, researchers to reconfigure it. Although there is a risk of oversimplifying a complex process this three-legged stool does have the capacity to create a picture of reality that acknowledges complex work and facilitates further creative discoveries and descriptions. 
So this stool is my gift. It is a gift back to all those wonderful midwives who took part in my study. It is a gift to all those whose research and ideas I read and pondered. But it is really a gift for all midwives and I wonder what they will make of it. Will it help them to understand what their practice is all about? What do their birth stools look like? What supports them in practice? What bits of their stools need attention? It is also a gift to the women for whom they care. Will it help them to understand what supports their midwife? They are at the centre of their midwife's care and the relationship she has with them is pivotal. But they need her to be well supported, well grounded in skilled and accountable care, able to work the system beside them and for them, able to be flexible and creative alongside them in the complex and uncertain journey into motherhood. It is also for educators and managers. This is what is important for midwives. This is how they make sense of their practice. How will they best prepare new practitioners for this role? How will they help students construct a birth stool that is strong and sturdy? How will managers provide systems and processes that encourage the midwife to use her stool and help her to keep it balanced and intact?

So let's return to where we started and sit for a while on the birth stool with Bronwen and Mele. What was my birth stool like and how did it support me? My seat was certainly 'being with' them both. My challenge with Bronwen was to manage the techno rational discourse in attempting to understand and communicate what her risks were and in managing my own fear around the repercussions of working outside the normal parameters of care. I had to understand and accept her position and mediate between her view, my own view and that of obstetrics; I had to be a paradigm broker. My 'being a professional' leg had a bit of a wobble but careful listening to her story and accepting her place, kept it firm. With Mele I was tossed off my birth stool for a while by a system that would not let me sit on it. However once I reconnected with Mele I worked the system and stayed 'with her' as I could, continuing to provide skilled care. I understood her cultural position, did not impose my own but was there for her when she needed me. While 'working the system' initially failed I was able to reconnect with it creatively and summon my skills and my patience to stay alongside her. In being with them both I built relationships that were precious, magic and loving. There is nothing quite like it! 
There are only two feelings. Love and fear.

There are only two languages. Love and fear.

There are only two activities. Love and fear.

There are only two motives, two procedures,

two frameworks, two results. Love and fear.

Love and Fear (Leunig, 1990). 


\section{Appendices}

Appendix A: Ethical approval 
Appendix B: Questionnaire 
Appendix C: Survey information letter 
Appendix D: Focus group information letter and consent forms 
Appendix E: Transcriber confidentiality form 


\section{References}

Alaszewski, H., \& Alaszewski, A. (1998). Professionals and practice: decision making and risk. In A. Alaszewski, L. Harrison \& J. Manthorpe (Eds.), Risk, health and welfare.Buckingham Philadelphia: Open University Press.

Albers, L. L. (1999). The duration of labor in healthy women. Journal of Perinatology, 19(2), 114-119.

Alexander, J. M., McIntire, D. D., \& Leveno, K. J. (2001). Prolonged pregnancy: induction of labor and cesarean births. Obstetrics and Gynecology, 97(6), 911915.

Alexander, S., \& Keirse, M. (Eds.). (1989). Effective care in pregnancy and childbirth.Buckingham: Open University Press.

Anderson, R., \& Murphy, P. (1995). Outcomes of 11788 planned home births attended by certified nurse-midwives. A retrospective descriptive study. Journal of Nurse Midwifery, 40(6), 483-492.

Annandale, E. (1996). Working on the front-line: risk culture and nursing in the new NHS. The Sociological Review, 44(3), 416-436.

Archer, M., Bhaskar, R., Collier, A., Lawson, T., \& Norrie, A. (Eds.). (1998). Critical realism.London: Routledge.

Archer, M., Sharp, R., Jones, R., \& Woodiwiss, T. (1999). Critical realism and research methodology. Alethia. International Association for Critical Realism, 2(1), 12-16.

Archer, M. S. (1995). Realist social theory: the morphogenetic approach.Cambridge: Cambridge University Press.

Arms, S. (1975). Immaculate deception.Boston: Bantam Book.

Asch, D., Jedrziewski, M., \& Christakis, N. (1997). Response rates to mail surveys published in medical journals. Journal of Clinical Epidemiology, 50(10), $1129-1136$.

Aslam, R. (1999). Risk management in midwifery practice. British Journal of Midwifery, 7(1), 41-44.

Australian College of Midwives. (2004). National midwifery guidelines for consultation and referral. Retrieved 22 March 2004, from http://www.acmi.org.au/test/corporate_documents/ref_guidelines.pdf

Banks, A. C. (1999). Birth chairs, midwives, and medicine.Jackson: University Press of Mississippi. 
Beck, U. (1999). World risk society.Malden: Polity Press.

Beck, U. (2000). Risk society revisited: theory, politics and research programmes. In U. Adam, 1. Beck \& J. v. Loon (Eds.), Risk society and beyond. Critical issues for social theory.London: Sage Publications.

Benjamin, Y., Walsh, D., \& Taub, N. (2001). A comparison of partnership caseload midwifery care with conventional team midwifery care: labour and birth outcomes. Midwifery, 17(3), 234-240.

Benoit, C. (1997). Professionalizing Canadian midwifery: sociological perspectives. In F. M. Shroff(Ed.), The new midwifery. Reflections on renaissance and regulation.

Bernstein, P. L. (1996). Against the Gods.New York: John Wliey \& Sons, Inc.

Bhaskar, R. (1978). A realist theory of science.Sussex: The Harvester Press Limited.

Bhaskar, R. (1986). Scientific realism and human emancipation.London: Verso.

Bhaskar, R. (1989). Reclaiming reality. A critical introduction to contemporary philosophy.London: Verso.

Bhaskar, R. (1991). Philosophy and the idea of freedom.Oxford: Blackwell.

Bhaskar, R. (1993). Dialectic. The pulse of freedom.London: Verso.

Biro, M., \& Lumley, J. (1991). The safety of team midwifery: the first decade of the Monash Birth Centre. Medical Journal of Australia, 155(7), 478-480.

Biro, M., Waldenstrom, U., \& Pannifex, J. H. (2000). Team midwifery care in a tertiary level obstetric service: a randomized controlled trial. Birth, 27(3), 168173.

Blanchette, H. (1995). Comparison of obstetric outcome of a primary-care access clinic staffed by certified nurse-midwives and a private practice group of obstetricians in the same community. American Journal of Obstetrics and Gynecology, 172(6), 1864-1868.

Blandford, J., \& Smyth, T. (2002). From risk management to clinical governance. In Mary G Harris and Associates (Ed.), Managing health services. Concepts and practice.Eastgardens, Australia: Maclennan and Petty.

Boulton, M., Chapple, J., \& Saunders, D. (2003). Evaluating a new service: clinical outcomes and women's assessment of the Edgeware Birth Centre. In M. Kirkham (Ed.), Birth centres. A social model for maternity care.Cheshire, England: Books for Midwives. 
Bourgeault, I. L., Declercq, E., \& Sandall, J. (2001). Changing birth. In R. De Vries, C. Benoit, E. R. van Teijlingen \& S. Wrede (Eds.), Birth by design.New York: Routledge.

Bradshaw, C., Lewis, P., \& Steer, P. (1995, May 1995). A midwifery team for high risk pregnancies. Modern Midwifery, 26-29.

Brink, P. J. (1991). Issues of reliability and validity. In J. M. Morse (Ed.), Qualitative nursing research.Newbury Park: Sage.

Brodie, P. (1996). Being with women: The experiences of Australian team midwives. Unpublished Masters of Nursing, University of Technology, Sydney.

Brownlee, M., Mcintosh, C., Wallace, E., \& Murphy-Black, T. (1996). A survey of interprofessional communication in a labour suite. British Journal of Midwifery, 4(9), 492-495.

Bryce, F. C., Clayton, J. K., Rand, R. J., Beck, I., Farquharson, D. I., \& Jones, S. E. (1990). General practitioner obstetrics in Bradford. British Medical Journal, 300(6726), 725-727.

Byrne, D. (2002). Interpreting quantitative data.London: Sage.

Calvert, S. (2002). Being with women. The midwife-woman relationship. In R. Mander \& V. Fleming (Eds.), Failure to progress. The contraction of the midwifery profession.London: Routledge.

Carey, M. A. (1994). The group effect in focus groups: planning, implementing, and interpreting focus group research. In J. M. Morse (Ed.), Critical issues in qualitative research methods.Thousand Oaks: Sage Publications.

Carroll, J. C., Reid, A. J., Ruderman, J., \& Murray, M. A. (1991). The influence of the high-risk care environment on the practice of low-risk obstetrics. Family Medicine, 23(3), 184-188.

Cartwright, E., \& Thomas, J. (2001). Constructing risk. Maternity care, law and malpractice. In R. De Vries, C. Benoit, E. R. van Teijlingen \& S. Wrede (Eds.), Birth by design. Pregnancy, maternity care, and midwifery in North America and Europe.New York: Routledge.

Cartwright, S. (1988). The Report of the Cervical Cancer Inquiry.Auckland: Government Printing Office.

Castel, R. (1991). From dangerousness to risk. In E. G. Burchell, C. Gordon \& P. Miller (Eds.), The Foucault effect.Herfordshire London: Harvester Wheatsheaf.

Chapman, M. G., Jones, M., Spring, J. E., DeSwiet, M., \& Chamberlain, G. V. P. (1986). The use of a birthroom: A randomised controlled trial comparing 
delivery with that in the labour ward. British Journal of Obstetrics and Gynaecology, 93, 182-187.

Cheyne, H., Turnbull, D., \& Lunan, C. (1995). Working alongside a midwife-led care unit: what do obstetricians think? British Journal of Obstetrics and Gynaecology, 10(6), 485-489.

College of Midwives of British Columbia. (1997). Indications for discussion, consultation and transfer of care.British Columbia: College of Midwives of British Columbia.

Collier, A. (1994). Critical realism. An introduction to Roy Bhaskar's philosophy.London: Verso.

Cooper, I. G. (2000). Clinical risk management. In D. Fraser (Ed.), Professional studies for midwifery practice.Edinburgh: Churchill Livingstone.

Craig, A. S., Berg, A. O., \& Kirkwood, C. R. (1985). Obstetric consultations during labor and delivery in a university-based family practice. Journal of Family Practice, 20(5), 481-485.

Crowley, P. (2001). Interventions to prevent or improve outcome from labour at or beyond term. The Cochrane Library(Update Software Issue 1).

Cunningham, W. (2004). The immediate and long-term impact on New Zealand doctors who receive patient complaints. The New Zealand Medical Journal, 117(1198).

Cunningham, W. (2004). The medical complaints and disciplinary process in New Zealand: doctors' suggestions for change. New Zealand Medical Journal, 117(1198).

Cunningham, W. (2004). New Zealand doctors' attitudes towards the complaints and disciplinary process. The New Zealand Medical Journal, 117(1198).

Danermark, B., Ekstrom, M., Jakobsen, L., \& Karlsson, J. (1997). Explaining society. Critical realism in the social sciences.London: Routledge.

Davenport, E. (2000). Clinical guidelines and the translation of texts into care: overcoming professional conflicts concerning evidence-based practice. Journal of Documentation, 56(5), 505-519.

Davis-FLoyd, R. (2002). Daughter of time:the postmodern midwife. Paper presented at the International Confederation of Midwives Conference, Vienna.

Davis, L., Reidmann, G., \& Sapiro, M. (1994). Cesarean section rates in low-risk private patients managed by certified nurse-midwives and obstetricians. Journal of Nurse-Midwifery, 39(2), 97-103.

de Vaus, D. A. (1995). Surveys in social research (Fourth ed.). London: Routledge. 
De Vries, R. (1996). The midwife's place: an international comparison of the status of midwives. In S. F. Murray (Ed.), Midwives and safer motherhood.London: Mosby.

De Vries, R. (2004). A pleasing birth. Midwives and maternity care in the Netherlands.Philadelphia: Temple University Press.

De Vries, R., \& Barroso, R. (2000). Midwives among the machines: recreating midwifery in the late 20th Century. Retrieved 24/03/2000, from http://www.stolaf.edu./stolaf/depts/sociology/devries/docs/midwifery.htm

De Vries, R., Salvensen, H., \& Wiegers, T. (2001). What (and why) do women want? The desires of women and the design of maternity care. In R. De Vries, C. Benoit, E. R. van Teijlingen \& S. Wrede (Eds.), Birth by design: pregnancy, maternity care and midwifery in North America and Europe.New York: Routledge.

Declercq, E. R. (1995). Midwifery care and medical complications: the role of risk screening. Birth, 22, 68-73.

Department of Health. (1993). Changing Childbirth. Report of the Expert Maternity Group.London: HMSO.

Donley, J. (1986). Save the midwife.Auckland, New Zealand: New Women's Press.

Donley, J. (1993). Independent midwifery. Paper presented at the Proceedings of the International Confederation of Midwives 23rd International Congress, Vancouver, Canada.

Donley, J. (1998). Birthrites: natural vs unnatural childbirth in New Zealand.Auckland, New Zealand: The Full Courts Press.

Douglas, M. (1992). Risk and blame: essays in cultural theory.London: Routledge.

Douglas, M., \& Wildavsky, A. (1982). Risk and culture: an essay on the selection of technical and environmental dangers.Berkeley: University of California Press.

Downe, S. (Ed.). (2004). Normal childbirth: evidence and debate.Edinburgh: Churchill Livingstone.

Duff, C., \& Sinclair, M. (2000). Exploring the risks associated with induction of labour: a retrospective study using the NIMATS database. Journal of Advanced Nursing, 31(2), 410-417.

Edwards, N. P. (2000). Women planning homebirths: their own views on their relationships with midwives. In M. Kirkham (Ed.), The midwife-mother relationship.London: Macmillan. 
Edwards, P., Roberts, I., Clarke, M., DiGuiseppi, C., Pratap, S., Wenz, R., et al. (2002). Increasing response rates to postal questionnaires: systematic review. British Medical Journal, 324(1183).

Enkin, M., Keirse, M., Neilson, J., Crowther, C., Duley, L., Hodnett, E., et al. (2000). A guide to effective care in pregnancy and childbirth.Oxford: Oxford University Press.

Fahy, K. (2002). Reflecting on practice to theorise empowerment for women: using Foucault's concepts. Australian Journal of Midwifery, 15(1), 5-13.

Fern, E. F. Advanced focus group research.

Fischhoff, B., \& Lichtenstein, S. (1981). Acceptable risk.Cambridge: Cambridge University Press.

Fleming, V. (2000). The midwifery partnership in New Zealand: past history or a new way forward. In M. Kirkham (Ed.), The midwife-mother relationship.London: Macmillan.

Flint, C., \& Poulengeris, P. (1987). The "Know your midwife" Report.Privately published: 49 Peckarman's Wood, Sydenham Hill, London SE26 6RZ.

Fogliani, M. (1999). Low response rates and their effects on survey results, 14/12/01, from

http://www.sch.abs.gov.au/SCH/A1610103.NSF/0/d28c3b1cab39524cca2569 a5007d153e/\$FILE/Low\%20Response\%20Rates.pdf F: survey

Foucault, M. (1991). Governmentality. In G. Burchell, C. Gordon \& P. Miller (Eds.), The Foucault effect.Brighton: Wheatsheaf.

Fowler, F. J. (1995). Improving survey questions. Design and evaluation. Thousnad Oaks, London, New Delhi: Sage Publications.

Fowler, F. J. (2002). Survey research methods.Thousand Oaks, California: Sage Publications.

Frankland, J., \& Bloor, M. (1999). Some issues arising in the systematic analysis of focus group materials. In R. S. Barbour \& J. Kitzinger (Eds.), Developing focus group research. Politics, theory and practice.London: Sage.

Fraser, S. W., \& Greenhalgh, T. (2001). Coping with complexity: educating for capability. British Medical Journal, 323(6 October), 700-803.

Fraser, W., Hatem-Asmar, M., Krauss, I., Maillard, F., Breart, G., \& Blais, R. (2000). Comparison of midwifery care to medical care in hospitals in the Quebec Pilot Projects Study: clinical indicators. Canadian Journal of Public Health, 91(1), 15-11. 
Freeman, L. M., Timperley, H., \& Adair, V. (2004). Partnership in midwifery care in New Zealand. Midwifery, 20, 2-14.

Giddens, A. (1999). Risk. Retrieved 18/04/99, from http://news.bbc.co.uk/hi/english/static/events/reith 99/week2/week2.htm

Gifford, D. S., Morton, S. C., Fiske, M., Keesey, J., Keller, E., \& Kahn, K. L. (2000). Lack of progress in labour as a reason for cesarean. Obstetrics and Gynecology, 95(4), 589-595.

Giles, W., Collins, J., Ong, F., \& MacDonald, R. (1992). Antenatal care of low risk obstetric patients by midwives. A randomised controlled trial. Medical Journal of Australia, 157(3), 158-161.

Glascoff, D. W. (2001). Physician response to surveys: a review of the literature. Marketing Health Services, 21(2), 39-41.

Greene, J. C., \& Caracelli, V. J. (2003). Making paradigmatic sense of mixed methods practice. In A. Tashakkori \& C. Teddlie (Eds.), Handbook of mixed methods in social and behavioural research.Thousand Oaks: Sage.

Griew, K. (2003). Birth centre midwifery down under. In M. Kirkham (Ed.), Birth centres. A social model for maternity care.Cheshire, England: Books for Midwives.

Guilliland, K. (1990). Women and midwives: a partnership in progress. Paper presented at the Proceedings of the International Confederation of Midwives 21 st International Congress, Kobe, Japan.

Guilliland, K. (1998). A demographic profile of independent (self-employed) midwives in NZ Aotearoa. Unpublished MA, Victoria University of Wellington, Wellington.

Guilliland, K., \& Pairman, S. (1995). The midwifery partnership: a model for practice.Wellington: Victoria University of Wellington, Monograph Series:95/1 Dept of Nursing and Midwifery.

Hacking, I. (1990). The taming of chance.Cambridge: Cambridge University Press.

Haertsh, M., Campbell, E., \& Sanson-Fisher, R. (1999). What is recommended for healthy women during pregnancy? A comparison of seven prenatal clinical practice guideline documents. Birth, 26(1), 24-30.

Hall, J. (2003). Free-standing maternity units in England. In M. Kirkham (Ed.), Birth centres. A social model for maternity care.Cheshire, England: Books for Midwives.

Harding, D. (2000). Making choices in childbirth. In L. Page (Ed.), The new midwifery: science and sensitivity in practice.Basingstoke: MacMillan Press. 
Harvey, S., Jarrell, J., Brant, R., Stainton, C., \& Rach, D. (1996). A randomized controlled trial of nurse-midwifery care. Birth, 23(3), 128-135.

Health and Disability Commissioner. (2004). Health and Disability Commissioner. Retrieved 5 October 2004, from http://www.hdc.org.nz/index.php

Health Funding Authority. (2000). Maternity services: a reference document.Hamilton, New Zealand: Ministry of Health.

Health Funding Authority Maternity Project Team. (1997). Guidelines for referral to obstetric and related specialist medical services. Wellington: Health Funding Authority.

Henderson, N. (2001). Making sense of litigation and clinical governance. British Journal of Midwifery, 9(4), 227-230.

Heyman, B. (Ed.). (1998). Risk, health and health care.London: Arnold.

Higginbotham, N., Albrecht, G., \& Connor, L. (2001). Health social science. A transdisciplinary complexity perspective.Oxford: Oxford University Press.

Homer, C. S., Davis, G. K., Brodie, P. M., Sheehan, A., Barclay, L. M., Wills, J., et al. (2001). Collaboration in maternity care: a randomised controlled trial comparing community-based continuity of care with standard hospital care. British Journal of Obstetrics and Gynaecology, 108(1), 16-22.

Homer, C. S., Matha, D. V., Jordan, L. G., Wills, J., \& Davis, G. K. (2001). Community-based continuity of midwifery care versus standard hospital care: a cost analysis. Australian Health Review, 24(1), 85-93.

Hundley, V., Cruickshank, F., Lang, G., Glazener, C., Milne, J., Turner, M., et al. (1994). Midwife managed delivery unit: a randomised controlled comparison with consultant care. British Medical Journal(309), 1400-1404.

Hunter, M. (2003). Autonomy, clinical freedom and responsibility. In M. Kirkham (Ed.), Birth centres. A social model for maternity care.Cheshire, England: Books for Midwives.

Janseen, P. A., Lee, S. K., Ryan, E. M., Etches, D. J., Farquharson, D. F., Peacock, D., et al. (2002). Outcomes of planned home births versus planned hospital births after regulation of midwifery in British Columbia. Canadian Medical Association Journal, 166(3).

Johanson, R., Newburn, M., \& Macfarlane, A. (2002). Has the medicalisation of childbirth gone too far? British Medical Journal, 324, 892-895.

Jordan, B. (1997). Authoritative knowledge and its construction. In R. E. Davis-Floyd \& C. F. Sargent (Eds.), Childbirth and authoritative knowledge. Crosscultural perspectives.Berkeley: University of California Press. 
Katz-Rothman, B. (2001). Spoiling the pregnancy. Prenatal diagnosis in the Netherlands. In R. De Vries, C. Benoit, E. R. van Teijlingen \& S. Wrede (Eds.), Birth by design.New York: Routledge.

Kean, L. H., Liu, D. T., \& Macquisten, S. (1996). Pregnancy care of the low risk woman: the community-hospital interface. International Journal of Health Care Quality Assurance, 9(5), 39-44.

Keaney, M. A. (1996). Is there a medical litigation crisis? Individual viewpoints on the perceived medical litigation crisis. Is litigation increasing? Medical Journal of Australia, 164, 178-179.

Kenny, P., Brodie, P., Eckerman, S., \& Hall, J. (1994). Westmead Hospital team midwifery project evaluation. Westmead, New South Wales, Australia: Centre for Health Economics Research and Evaluation, Westmead Hospital.

Kent, J. (2000). Social persepctives on pregnancy and childbirth for midwives, nurses and the caring professions.Buckingham: Open University Press.

Kent, J. (2000). Social perspectives on pregnancy and childbirth for midwives, nurses and the caring professions.Buckingham: Open University Press.

Kirkham, M. (1996). Professionalization past and present: with women or with the powers that be? In D. Kroll (Ed.), Midwifery care for the future. Meeting the challenge.London: Bailliere Tindall.

Kirkham, M. (1999). The culture of midwifery in the national health service in England. Journal of Advanced Nursing, 30, 732-739.

Kirkham, M. (Ed.). (2000). The midwife-mother relationship.London: Macmillan.

Kirkham, M. J. (1997). Stories and childbirth. In M. J. Kirkham \& E. R. Perkins (Eds.), Reflections on midwifery.London: Bailliere Tindall.

Kitzinger, J. (1994). The methodology of focus groups: the importance of interaction between research participants. Sociol Health \& Illness, 16(1), 103-121.

Kitzinger, J., \& Barbour, R. S. (1999). The challenge and promise of focus groups. In R. S. Barbour \& J. Kitzinger (Eds.), Developing focus group research.London: Sage.

Kitzinger, J., Green, J., \& Coupland, V. (1990). Labour relations: midwives and doctors on the labour ward. In J. Garcia, R. Kilpatrick \& M. Edwards (Eds.), The politics of maternity care. Services for childbearing women in twentiethcentury Britain.Oxford: Oxford University Press.

Klein, M., Llloyd, I., Redman, C., Bull, M., \& Turnbull, A. C. (1983). Comparison of low risk pregnant women booked for delivery in two systems of care: sharedcare (consultant) and integrated general practice unit. 1 Obstetrical procedures 
and neonatal outcomes. British Journal of Obstetrics and Gynaecology, 90, 118-122.

Klein, M., Spence, A., Kaczorowski, J., Kelly, A., \& Grzzybowski, S. (2002). Does delivery volume of family physicians predict maternal and newborn outcome? Canadian Medical Association Journal, May14.

Knodel, J. (1993). The design and analysis of focus group studies. In D. L. Morgan (Ed.), Successful focus groups. Advancing the state of the art.Newbury Park: Sage.

Knox, A. J., Sadler, L., \& Patterson, N. S. (1993). An obstetric scoring system: its development and application in obstetric management. Obstetrics and Gynecology, 81, 195-199.

Krueger, R. (1994). Focus groups: a practical guide for applied research.Beverly Hills, CA: Sage.

Krueger, R. A. (1998). Moderating focus groups.Thousand Oaks: Sage.

Lane, K. (1995). The medical model of the body as a site of risk: a case of childbirth. In J. Gabe (Ed.), Medicine, health and risk. Sociological approaches.Oxford: Blackwell Publishers.

Langford, J., \& McDonagh, D. (2003). Introduction. In J. Langford \& D. McDonagh (Eds.), Focus Groups.London: Taylor and Frances.

Lauchland, M. (1996). The shared journey: models in midwifery practice. New Zealand College of Midwives Journal, 14, 24-27.

Law, Y. Y., \& Lam, K. Y. (1999). A randomized controlled trial comparing midwifemanaged care and obstetrician-managed care for women assessed to be at low risk in the initial intrapartum period. Journal of Obstetric and Gynaecological Research, 25(2), 107-112.

Lay, M. M. (2000). The rhetoric of midwifery gender, knowledge and power. New Brunswick,New Jersey,London: Rutgers University Press.

Leap, N. (2000). 'The less we do, the more we give'. In M. Kirkham (Ed.), The midwife-mother relationship.London: Macmillan.

Leape, L., \& Berwick, D. (2002). Safe health care: are we up to it? British Medical Journal, 320, 725-726.

LeFevre, M. L. (1992). Physician volume and obstetric outcome. Medical Care, 30(9), 866-871.

Leunig, M. (1990). A Common Prayer. A cartoonist talks to Go:HarperCollins Publishers. 
Linde, C. (1993). Life Stories.Oxford: Oxford University Press.

Lupton, D. (1999). Risk.London: Routledge.

MacVicar, J., Dobbie, G., Owen-Johnstone, L., Jagger, C., Hopkins, M., \& Kennedy, J. (1993). Simulated home delivery in hospital: a randomised controlled trial. British Journal of Obstetrics and Gynaecology, 100, 316-323.

Mander, R. (2002). The midwife and the medical practitioner. In R. Mander \& V. Fleming (Eds.), Failure to progress. The contraction of the midwifery profession.London, New York: Routledge.

Maslow, A. S., \& Sweeney, A. L. (2000). Elective induction of labor as a risk factor for cesarean delivery among low-risk women at term. Obstetrics and Gynecology, 95, 917-922.

Maternal and Newborn Health/Safe Motherhood Unit. (1996). Care in normal birth: a practical guide.Geneva: World Health Organization.

May, K. A. (1994). Abstract knowing: the case for magic in method. In J. M. Morse (Ed.), Critical issues in qualitative research methods. Thousand Oaks: Sage.

McDrury, J., \& Alterio, M. (2002). Learning through storytelling: using reflection and experience in higher education contexts.Palmerston North, New Zealand: The Dunmore Press Limited.

Mckay, S. (2000). Models of midwifery care: Denmark, Sweden, and The Netherlands. In E. v. Teijlingen, G. Lowis, P. McCaffery \& M. Porter (Eds.), Midwifery and the medicalization of childbirth: comparative perspectives.Huntington, New York: Nova Science Publishers, Inc.

Mead, M., \& Kornbrot, D. (2004). The influence of maternity units' intrapartum intervention rates and midwives' risk perception for women suitable for midwifery-led care. Midwifery, 20, 61-71.

Menand, L. (2002). The metaphysical club.London: Flamingo.

Menticoglou, S. M., \& Hall, P. H. (2002). Routine induction of labour at 41 weeks gestation: nonsensus consensus. British Journal of Obstetrics and Gynaecology, 109(5), 485-491.

Merton, R., Fiske, M., \& Kendall, P. (1990). The focused interview: A manual of problems and procedures (2nd Edition ed.). New York: Free Press.

Ministry of Health. (1996). Notice issued pursuant to Section 51 of the Health and Disability Services Act 1993 concerning the provision of maternity services. Wellington New Zealand. 
Ministry of Health. (2002). Maternity Services. Notice pursuant to Section 88 of the New Zealand Public Health and Disability Act 2000.Wellingon: Ministry of Health.

Ministry of Health. (2002). Notice pursuant to Section 88 of the New Zealand Public Health and Disability Act 2000.Wellington: Ministry of Health.

Mohamed, H., Martin, C., \& Haloob, R. (2002). Can the New Zealand antenatal scoring system be applied in the United Kingdom? Journal of Obstetrics and Gynaecology, 22(4), 389-391.

Morgan, D. L. (1988). Focus groups as qualitative research (Second Edition ed. Vol. 16). Thousand Oaks: Sage.

Morgan, D. L. (1998). Planning focus groups.Thousand Oaks: Sage.

Morgan, D. L., \& Krueger, R. A. (1993). When to use focus groups and why. In D. L. Morgan (Ed.), Successful focus groups. Advancing the state of the art.Newbury Park: Sage.

Morse, J. M. (1994). "Emerging from the data"- The cognitive processes of analysis in qualitative inquiry. In J. M. Morse (Ed.), Critical issues in qualitative research methods.Thousand Oaks: Sage.

Morse, J. M. (2003). Principles of mixed methods and multimethod research design. In A. Tashakkori \& C. Teddlie (Eds.), Handbook of mixed methods in social and behavioural research.Thousand Oaks: Sage.

Murphy-Lawless, J. (1998). Reading birth and death: a history of obstetric thinking.Bloomington and Indianapolis: Indiana University Press.

Myers, G., \& Macnaghten, P. (1999). Can focus groups be analysed as talk? In R. S. Barbour \& J. Kitzinger (Eds.), Developing focus group research. Politics, theory and practice.London: Sage.

National Health Committee. (1999). Review of maternity services in New Zealand.Wellington: National Advisory Committee on Health and Disability.

National Health Insurance Board of the Netherlands. (1998). Obstetric Manual: final report of the obstetric working group of the National Health Insurance Board of the Netherlands. Retrieved 24/7/2003, 2003, from http://europe.obgyn.net/nederland/?page=/nederland/richtlijnen/vademecum_e ng $\sec 5$

New Zealand College of Midwives Inc. (1993). Midwives handbook for practice:The New Zealand College of Midwives.

New Zealand Guidelines Group. (2004). Care of women with breech presentation or previous caesarean birth. Wellington: New Zealand Guidelines Group. 
New Zealand Health Information Service. (2001). New Zealand Workforce Statistics 2001. Retrieved 23/11/01, from http://www.nzhis.govt.nz/stats/nursestats.html

New Zealand Health Information Service. (2003). Report on maternity 2000 \&2001.Wellington: New Zealand Health Information Service.

New Zealand Health Information Service. (2004). Report on Maternity 2002. Maternal and newborn information. Wellington: New Zealand Health Information Service.

O'Donnell, C. (2000). Variation in GP referral rates: what can we learn from the literature? Family Practice, 17(6), 462-471.

Oakley, A. (2000). Experiments in knowing: gender and method in the social sciences.Cambridge: Polity.

Oakley, A., \& Houd, S. (1990). Helpers in childbirth midwifery today.New York: Hemisphere Publishing Corporation.

Oakley, D., Murray, M., \& Murtland, T. (1996). Processes of care; comparisons of certified nurse-midwives and obstetricians. Journal of Nurse Midwifery, 40(5), 399-409.

Olsson, P., Jansson, L., \& Norberg, A. (2000). A qualitative study of childbirth as spoken about in midwives' ante- and postnatal consultations. Midwifery, 16(123-134).

Page, L. (Ed.). (1995). Effective group practice in midwifery: working with women.Oxford, London: Blackwell Science.

Page, L., McCourt, C., Beake, S., Vail, A., \& Hewison, J. (1999). Clinical interventions and outcomes of One-to-One midwifery practice. Journal of Public Health Medicine, 21(3), 243-248.

Page, L., \& Percival, P. (Eds.). (2000). The new midwifery: science and sensitivity in practice.Edinburgh: Churchill Livingstone.

Pairman, S. (2000). Women-centred midwifery: partnerships or professional friendships? In M. Kirkham (Ed.), The midwife-mother relationship.London: Macmillan.

Pairman, S., \& Guilliland, K. (2003). Developing a midwife-led maternity service: the New Zealand experience. In M. Kirkham (Ed.), Birth centres. A social model for maternity care. Cheshire, England: Books for Midwives.

Papps, E., \& Olssen, M. (1997). Doctoring childbirth and regulating midwifery in New Zealand: a Foucauldian perspective.Palmerston North, NZ: Dunmore Press. 
Pasveer, B., \& Akrich, M. (2001). Obstetrical trajectories. On training women/bodies for (home) birth. In R. De Vries, C. Benoit, E. R. van Teijlingen \& S. Wrede (Eds.), Birth by design.New York: Routledge.

Platt, L. D., Angelini, D. J., Paul, R. H., \& Quilligan, E. J. (1985). Nurse-midwifery in a large teaching hospital. Obstet Gynecol, 66(6), 816-820.

Plsek, P. E., \& Greenhalgh, T. (2001). The challenge of complexity in health care. British Medical Journal, 323(7313), 625.

Porter, S. (2002). Critical realist ethnography. In T. May (Ed.), Qualitative research in action.London: Sage.

Radomsky, N. A. (1995). Family practice obstetrics in a community hospital. Canadian Family Physician, 41, 617-624.

Reason, P., \& Goodwin, B. (1999). Toward a science of qualities in organisations: Lessons from complexity theory and postmodern biology. Concepts and Transformation, 4(3), 281-317.

Reed, J., \& Roskell, V. (1997). Focus groups: issues of analysis and interpretation. Journal of Advanced Nursing, 26, 758-764.

Reid, A. J., Carroll, J. C., Ruderman, J., \& Murray, M. (1995). Consultation in family practice obstetrics. Canadian Family Physician, 41, 591-598.

Rich, A. (1976). Of woman born:motherhood as experience and institution.London: Virago.

Richard, L. (2001). Making childbirth a normal process. Birth, 28(1), 1-3.

Rohde, J. E. (1995). Removing risk from safe motherhood. International Journal of Gynecology \& Obstetrics, 50(2), S3-S10.

Rooks, J. P. (1997). Midwifery and childbirth in America.Philadelphia: Temple University Press.

Rooney, C. (1992). Antenatal care and maternal health: how effective is it? A review of the evidence.Geneva: World Health Organisation.

Rosenblatt, R., Dobie, S., \& Hart, L. (1997). Interspecialty differences in the obstetric care of low-risk women. American Journal of Public Health, 87(3), 344-351.

Rosenblatt, R. A., Reinken, J., \& Shoemack, P. (1985). Is obstetrics safe in small hospitals? Lancet, 2(8452), 429-432.

Rosenburg, K., \& Trevathan, W. (2002). Birth, obstetrics and human evolution. International Journal of Obstetrics and Gynecology, 109, 1199-1206. 
Rosenthal, T. C., Holden, D. M., \& Woodward, W. (1990). Primary care obstetrics in rural western New York. A multi-center case review. New York State Journal of Medicine, 90(11), 537-540.

Rowlands, G., \& Lewis, G. (2001). Risky business. Health Services Journal, 111(5757), 28-29.

Rowley, M. J., Hensley, M. J., Brinsmead, M. W., \& Wlodarczyk, J. H. (1995). Continuity of care by a midwife team versus routine care during pregnancy and birth: a randomised trial. Medical Journal of Australia, 163(6), 289-293.

Sandall, J. (1997). Midwives' burnout and continuity of care. British Journal of Midwifery, 5, 106-111.

Sandall, J. (2000). Choice, continuity and control: changing midwifery towards a sociological perspective. In E. V. Teijlingen, G. Lowis, P. McCaffery \& M. Porter (Eds.), Midwifery and the medicalisation of childbirth: comparitive perspectives.New York: Nova Science Publishers.

Sandall, J., Davies, J., \& Warwick, C. (2001). Evaluation of the Albany Midwifery Practice. Final Report March 2001.London: King's College London.

Saxell, L. (2000). Risk: theoretical or actual. In L. Page, P. Percival \& S. Kitzinger (Eds.), The new midwifery.Edinburgh London: Churchill Livingstone.

Sayer, A. (1992). Method in social science: a realist approach.London: Routledge.

Sayer, A. (2000). Realism and social science.London: Sage.

Schettler, T., Solomon, G., Valenti, M., \& Huddle, A. (1999). The role of science in public health decisions. In Generations at risk: reproductive health and the environment.Cambridge, Massachusetts, London, England: The MIT Press.

Schon, D. A. (1983). The reflective practitioner.New York: Basic Books.

Shroff, F. M. (Ed.). (1997). The new midwifery: reflections on renaissance and regulation.

Sim, J. (1998). Collecting and analysing qualitative data: issues raised by the focus group. Journal of Advanced Nursing, 28(2), 345-352.

Skinner, J. (1999). Midwifery partnership: individualism, contractualism or feminist praxis? NZ College of Midwives(21), 14-17.

Skinner, J. (2002). Love and Fear. The midwife and her relationship with risk. Birth Issues, 11(2/3), 69-72.

Smit, Y., Scherjon, S. A., \& Treffers, P. E. (1997). Elderly nulliparae in midwifery care in Amsterdam. Midwifery, 13(2), 73-77. 
Smith, G. C., Pell, J. P., \& Dobbie, R. (2002). Birth order, gestational age and risk of delivery related perinatal death in twins: retrospective cohort study. Retrieved 4/11/2002, 2002, from http://bmj.com/cgi/content/full/325/7371/1004

Smith, L. F. P. (1998). Referral of labouring women from community to consultant unit. British Journal of Midwifery, Jan 6(1), 47-49, 51-42.

Smythe, E. (1998). "Being safe' in childbirth: a hermeneutic interpretation of the narratives of women and practitioners. Unpublished $\mathrm{PhD}$, Massey University, New Zealand.

Smythe, L. (2000). Being safe in childbirth; what does it mean? New Zealand College of Midwives Journal(22), 18-21.

Spencer, N. (2002). The rediscovery of reality. Retrieved 1/11/02, from http://www.raggedclaws.com/criticalrealism/archive/nspencer_rr.html

Stahl, K., \& Hundley, V. (2003). Risk and risk assessment in pregnancy - do we scare because we care? Midwifery, 19, 298-309.

Standards New Zealand. (2001). Sentinel events workbook:Ministry of Health.

Stapleton, H. (1997). Choice in the face of uncertainty. In M. J. Kirkham \& E. R. Perkins (Eds.), Reflections on midwifery.London: Bailliere Tindall.

Stapleton, H., Kirkham, M., \& Thomas, G. (2002). Qualitative study of evidence based leaflets in maternity care. $B M J, 323,634$.

Statistics New Zealand. (2004). Key demographic indicators. Retrieved 1/2/05, from http://www.stats/govt.nz/popn-monitor/key-indicators/default.htm

Stern, P. N. (1994). Eroding grounded theory. In J. M. Morse (Ed.), Critical issues in qualitative research methods.Thousand Oaks: Sage.

Stewart, D. W., \& Shamdasani, P. N. (1990). Focus groups. Theory and practice (Vol. 20). Newbury Park: Sage Publications.

Stewart, M., Brown, J. B., Weston, W. W., McWhinney, I., McWilliam, C., \& Freeman, T. (2003). Patient-centred medicine. Transforming the clinical method.Oxon: Radcliffe Medical Press.

Street, P., Gannon, M. J., \& Holt, E. M. (1991). Community obstetric care in West Berkshire. British Medical Journal, 302(6778), 698-700.

Symon, A. (1998). Litigation; the views of midwives and obstetricians.Cheshire, England: Hochland \& Hochland Ltd.

Symon, A. (2000). Litigation and changes in professional behaviour: quantifying the problem. Midwifery, 16(1), 15-21. 
Symon, A., \& Wilson, J. (2002). The way forward: clinical competence, co-operation and communication. In J. Wilson \& A. Symon (Eds.), Clinical risk management in midwifery. The right to a perfect baby. Oxford: Books for Midwives.

Taylor, R. (2004). Midwifery, professional misconduct and Codes of Ethics: an analysis of findings by The Accident Corporation, the Health and Disabilities Commission and the New Zealand Nursing Council. Paper presented at the New Zealand College of Midwives 8th Biennial National Conference, Wellington.

Tew, M. (1985). Place of birth and perinatal mortality. Journal of the Royal College of General Practitioners, 35, 390-394.

Tew, M. (1995). Safer childbirth? A critical history of maternity care (Second ed.). London, UK: Chapman \& Hall.

The National Health Committee. (1999). Review of maternity services in New Zealand.Wellington, New Zealand: National Advisory Committee on Health and Disability.

Thomas, B. G. (2000). Be nice and don't drop the baby. In L. A. Page (Ed.), The new midwifery. Science and sensitivity in practice.Edinburgh: Churchill Livingstone.

Thomas, P. (Ed.). (2002). The midwife you have called knows you are waiting. A consumer view.London: Routledge.

Thompson, M. (2002). Intra- and inter-professinal behaviour and communication. In J. H. Wilson \& A. Symon (Eds.), Clinical risk management in midwifery. The right to a perfect baby. Oxford: Books for Midwives.

Tilyard, M. W., Williams, S., Seddon, R. J., Oakley, M. E., \& Murdoch, C. J. (1988). Is outcome for general practitioner obstetricians influenced by workload and locality? New Zealand Medical Journal, 101(844), 207-209.

Tucker, J. S., Hall, M. H., Howie, P. W., Reid, M. E., Barbour, R. S., Florey, C. D., et al. (1996). Should obstetricians see women with normal pregnancies? A multicentre randomised controlled trial of routine antenatal care by general practitioners and midwives compared with shared care led by obstetricians. British Medical Journal, 312(7030), 554-559.

Tully, E. (1999). Doing professionalism differently: negotiating midwifery autonomy in Aotearoa/New Zealand. Unpublished PhD, University of Canturbury, Christchurch. New Zealand.

Tully, L., Daellenbach, R., \& Guilliland, K. (1998). Feminism, partnership and midwifery. In R. d. Plessis \& L. Alice (Eds.), Feminist thought in Aotearoa/New Zealand.Auckland: Oxford Universtiy Press. 
Turnbull, D., Holmes, A., \& Shields, N. (1996). Randomised, controlled trial of efficacy of midwife-managed care. Lancet, 348, 213-218.

Turner, B. S. (1997). From governmentality to risk. Some reflections on Foucault's contribution to medical sociology. In A. Petersen \& R. Bunton (Eds.), Foucault, health and medicine.London: Routledge.

Tyson, H. (1991). Outcomes of 1001 midwife-attended home births in Toronto. 19831988. Birth, 18, 14-19.

van der Hulst, L. A. M., van Teijlingen, E. R., Bonsel, G. J., Eskes, M., \& Bleker, O. P. (2004). Does a Pregnant Woman's Intended Place of Birth Influence Her Attitudes Toward and Occurrence of Obstetric Interventions? Birth, 31(1), 2833.

Virtue, C. (2000). Annual Review. Domino Midwives Wellington. Nga Tapuhi Whakawhanau.1999-2000.Wellington New Zealand: Domino Midwives.

Voelk1, K. E., \& Gerber, S. B. (1999). Using SPSS for Windows: data analysis and graphics.New York: Springer.

Wagner, M. (1994). Pursuing the birth machine.Camperdown, Australia: ACE Graphics.

Waldenstrom, U., \& Turnbull, D. (1998). A systematic review comparing continuity of midwifery care with standard maternity services. British Journal of Obstetrics and Gynaecology, 105(11), 1160-1170.

Walker, J. F. (1976). Midwife or obstetric nurse? Some perceptions of midwives and obstetricians of the role of the midwife. Journal of Advanced Nursing, 1, 129138 .

Wallace, E., Mcintosh, C., Brownlee, M., Laidlaw, 1., \& Johnstone, F. (1995). An observational study of midwife-medical staff interaction in a labour ward environment. Journal of Obstetrics and Gynaecology, 15, 165-170.

Walsh, D., El-Nemer, A., \& Downe, S. (2004). Risk, safety and the study of physiological birth. In S. Downe (Ed.), Normal childbirth: evidence and debate.Edinburgh: Churchill Livingstone.

Waterton, C., \& Wynne, B. (1993). Can focus groups access community views? In R. S. Barbour \& J. Kitzinger (Eds.), Developing focus group research.London: Sage.

Wiegers, T. A., Keirse, M. J., van der Zee, J., \& Berghs, G. A. (1996). Outcome of planned home and planned hospital births in low risk pregnancies: prospective study in midwifery practices in The Netherlands. British Medical Journal, 313(7068), 1309-1313. 
Wiegers, T. A., van der Zee, J., \& Keirse, M. J. (1998). Maternity care in The Netherlands: the changing home birth rate. Birth, 25(3), 190-197.

Wilkins, R. (2000). Poor relations; the paucity of the professional paradigm. In M. Kirkham (Ed.), The midwife-mother relationship.London: Macmillan.

Wilkinson, I. (2001). Anxiety in a risk society.London: Routledge.

Wilkinson, S. (1999). How useful are focus groups in feminist research? In R. S. Barbour \& J. Kitzinger (Eds.), Developing focus group research.London: Sage.

Williams, M. (2002). Generalization in interpretive research. In T. May (Ed.), Qualitative research in action.London: Sage.

Williams, T. (2002). Two research fallacies. Retrieved 11/2/05, from http://www/socialresearchmethods.net/kb/fallacy.htm

Wilson, J. (2002). Principles of clinical governance. In J. H. Wilson \& A. Symon (Eds.), Clinical risk management in midwifery. The right to a perfect baby.Oxford: Books for Midwives.

Wilson, J., \& Symon, A. (Eds.). (2002). Clinical risk management in midwifery. The right to a perfect baby. Oxford: Books for Midwives.

Witz, A. (1992). Professions and patriarchy.London: Routledge.

Wolff, B., Knodel, J., \& Sittitrai, W. (1993). Focus groups and surveys as complementary reserach methods. In D. L. Morgan (Ed.), Successful focus groups.Newbury Park: Sage.

World Health Organisation. (1994). Indicators to monitor maternal health goals: report of a technical working group.Geneva.

World Health Organisation. (1998). World health day 1998: every pregnancy faces risk. Retrieved 11/04/2001, from http://www.who.int/archives/whday/en/pages1998/whd98 05.html

World Health Organisation. (2004). Care in normal birth: report of a technical working group. Retrieved 30/4/04, from http://www.who.int/reproductivehealth/publications/MSM_96_24/MSM_96_24_table_of_contents.en.html

Yuster, E. A. (1995). Rethinking the role of the risk approach and antenatal care in maternal mortality reduction. International Journal of Gynecology \& Obstetrics, 50(2), S59-S61.

Zhang, J., Troendle, J. F., \& Yancey, M. K. (2002). Reassessing the labor curve in nulliparous women. American Journal of Obstetrics and Gynecology, 187(4), 824-828. 
Our Ref 01/03/019

Please include the reference number and study title in all correspondence.

Joan Skinner

10A London Road

Korokoro

Lower Hutt

Dear Joan \\ Ethics Committee}

Room 425, Fourth Floor

community \& support services

Wellington Hospital

Private Bag 7902

Wellington South

Phone (04) 3855999 ext. 5185

Fax (04) 3855840

Email: claire.l@wec.org.nz

\section{1/03/019 - The role of the midwife in caring for women experiencing complex pregnancies. Multicentre study - Wellington Lead}

Thank you for your letter of 17 April, responding to my letter of 6 April and enclosing a paper on methodology selection, and amended information letters for both the focus group and the national survey.

I also appreciated the further opportunity to talk with you today about the status of discussions with appropriate cultural advisors. You advise that you have been in contact with Irihapeti Ramsden and are having ongoing discussions with her about the cultural implications that arise from your research, particularly those issues around risk. You will conduct a focus group for Maori midwifes, should there be enough in any one locality and if this does occur, there will be further consultation with Irihapeti and Liz Loschman-Ellison. Your point about possible distortion of the flow and interpretation of the group discussion is noted if focus group participants are later allowed to amend transcript. It is preferable to make the transcripts available but in order to avoid this distortion, if participants were unhappy with the transcript then they should have the right to withdraw from the study and have their comments deleted from the record.

As your response has satisfactorily addressed the various issues raised in my letter and you have made the requested amendments to the information sheets and the questionnaire, final ethical approval for the above study is granted by the Chairperson under delegated authority from the Wellington Ethics Committee on behalf of all other regional ethics committees. It is a condition of Ethics Committee approval that you provide a brief progress report no later than April 2002 and at the completion of the study a copy of any report/publication for the Committee's records. Please notify the Committee if the study is abandoned or changed in any way.

We wish you well with your research.

Yours sincerely

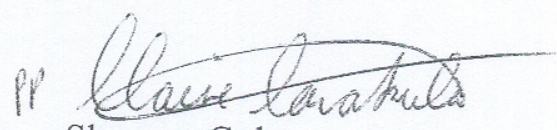

Sharron Cole

CHAIRPERSON

cc Regional Ethics Committees 


\section{THE MIDWIFE with RISK - SURVEY FORM}

Your details:

Your year of birth: 1 Your year of registration as a midwife: 2

Where were you first registered as a midwife? (tick one only):

$3 \square$ New Zealand

\begin{tabular}{|l|l|l}
6 & $\square$ South Africa \\
7 & $\square$ UK \\
8 & Europe
\end{tabular}

$9 \quad \square$ Asia
$10 \square$ Pacific
$11 \square$ Other

What other types of registration do you hold? (tick as many as apply)

\begin{tabular}{|c|c|c|}
\hline $12 \square$ General Nurse & Comprehensive Nurse & $17 \square$ Obstetric Nurse \\
\hline $\begin{array}{l}\text { General and } \\
\text { Obstetric Nurse }\end{array}$ & $\begin{array}{l}15 \square \text { Enrolled Nurse } \\
16 \square \text { Psychiatric Nurse }\end{array}$ & $18 \quad$ Other \\
\hline
\end{tabular}

How many years have you been in practice as a midwife? 19

Type of midwifery that you offer to women (tick one or more)

\begin{tabular}{|c|c|}
\hline FULL CARE & PART CARE \\
\hline 20 Total Midwifery & $25[$ Postnatal only \\
\hline $21 \square$ Shared care with hospital midwives & $\begin{array}{l}26 \quad \begin{array}{l}\text { Antenatal \& } \\
\text { postnatal only }\end{array} \\
\end{array}$ \\
\hline $22 \sqcup$ Shared care with GP (A/N, labour, birth, P/N) & $\begin{array}{l}27 \text { Labour and birth } \\
\text { support only }\end{array}$ \\
\hline $\begin{array}{l}23 \text { Shared care with O\&G (A/N, labour, birth, } \\
\text { P/N) }\end{array}$ & $28 \square$ Other - please state \\
\hline $\begin{array}{l}24 \square \text { Shared care for } \mathrm{A} / \mathrm{N} \text {, midwifery only labour \& } \\
\mathrm{P} / \mathrm{N}\end{array}$ & \\
\hline
\end{tabular}

Which of the above is used by the largest proportion of your clients?

(enter one number between 20 and 28 as appropriate)

29

What is your present form of funding? (tick one only)

$30 \square$ Direct Section 88 claimant

$31 \square$ Midwifery Provider Organisation/other form of non section 88 claimant

$32 \square$ Employee of DHB (hospital)

$33 \square$ Employee of School of Midwifery/Nursing

34] Employee of General

Practitioner/Medical Agency

35 Employee of Community

Owned Provider/Trust/Iwi

36[] Other (please state) 
Main hospital to which your obstetric referrals are made and/or secondary care provided. (Tick one only)

$37 \square$ Northland Base
$38 \square$ North Shore
$39 \square$ Waitakere
$40 \square$ National Wms
$41 \square$ Middlemore
$42 \square$ Waikato
$43 \square$ Tauranga
$44 \square$ Whakatane

$$
\begin{array}{|l|l}
45 \square & \text { Gisborne } \\
46 \square & \text { Rotorua } \\
47 \square & \text { Taranaki Base } \\
48 \square & \text { Wanganui } \\
49 \square & \text { Palmerston North } \\
\mathbf{5 0} \square \text { Hastings } \\
\mathbf{5 1} \square \text { Masterton } \\
\mathbf{5 2} \square \text { Hutt }
\end{array}
$$

$\mathbf{5 3} \square$ Wellington Wms
$\mathbf{5 4} \square$ Wairau
$\mathbf{5 5} \square$ Nelson
$\mathbf{5 6} \square$ Christchurch Wms
$\mathbf{5 7} \square$ Greymouth
$\mathbf{5 8} \square$ Timaru
$\mathbf{5 9} \square$ Dunedin
$\mathbf{6 0} \square$ Southland

What is the distance in kms from your home to this hospital?

61

\section{Data Collection Sheet}

Please give the requested data for all women you booked who were due to deliver in the four months from 1 September 2000 - 31 December 2000 and who you referred for obstetric consultation or who had care transferred to an obstetrician.

Number of clients who had referrals or transfer of care in these four months

62<smiles>C1CCC1</smiles>

Total number of clients you booked who were due to deliver in these four months

63

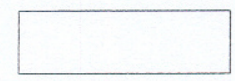

Please include on the data collection sheet only those women who had a referral and/or had transfer of care to an obstetrician at any stage of the childbearing process. If you had no referrals your answers to all the other questions are still important. Please complete them and return the questionnaire.

(Referral means a consultation with an obstetrician while you are LMC. Transfer of care occurs where either clinical responsibility or LMC has been transferred to an obstetrician.)

\begin{tabular}{|c|c|c|c|c|}
\hline \multicolumn{5}{|c|}{ CODING for Data Collection Sheet } \\
\hline \multicolumn{3}{|c|}{ Reason for $\mathrm{A} / \mathrm{N}$ or $\mathrm{P} / \mathrm{N}$} & \multicolumn{2}{|c|}{ Reasons for Labour and Birth } \\
\hline \multicolumn{2}{|c|}{ Consultation or Transfer } & & & Consultation or Transfer \\
\hline apa & APH/abruption & & $\mathrm{bch}$ & Breech \\
\hline app & APH/Praevia & & $\mathrm{cpd}$ & $\mathrm{CPD}$ \\
\hline bch & Breech & & fdr & Foetal Distress \\
\hline inf & Infection & & idl & Instrumental Delivery \\
\hline iud & IUD & & inf & Infection \\
\hline iug & IUGR & & $\mathrm{iph}$ & Haemorrhage \\
\hline med & Pre-existing medical condition & & lop & Lack of progress \\
\hline mis & Miscarriage & & nvx & Non-vertex, unstable lie \\
\hline mul & Multiple pregnancy & & & \\
\hline navx & Non-vertex, unstable lie & & & \\
\hline pet & Pre-eclampsia & \multicolumn{3}{|c|}{ Who the Referral was to: } \\
\hline $\mathrm{pph}$ & PPH (over $500 \mathrm{ml}$ ) & \multirow{3}{*}{\multicolumn{3}{|c|}{$\begin{array}{ll}\text { hos } & \text { Hospital clinic/Maternity team } \\
\text { pte } & \text { Obstetrician - where the client pays a fee } \\
\text { pub } & \text { Obstetrician - where the client does not pay a fee }\end{array}$}} \\
\hline pre & Premature labour & & & \\
\hline pro & Prolonged pregnancy & & & \\
\hline prm & Prolonged rupture of membranes & & & \\
\hline
\end{tabular}



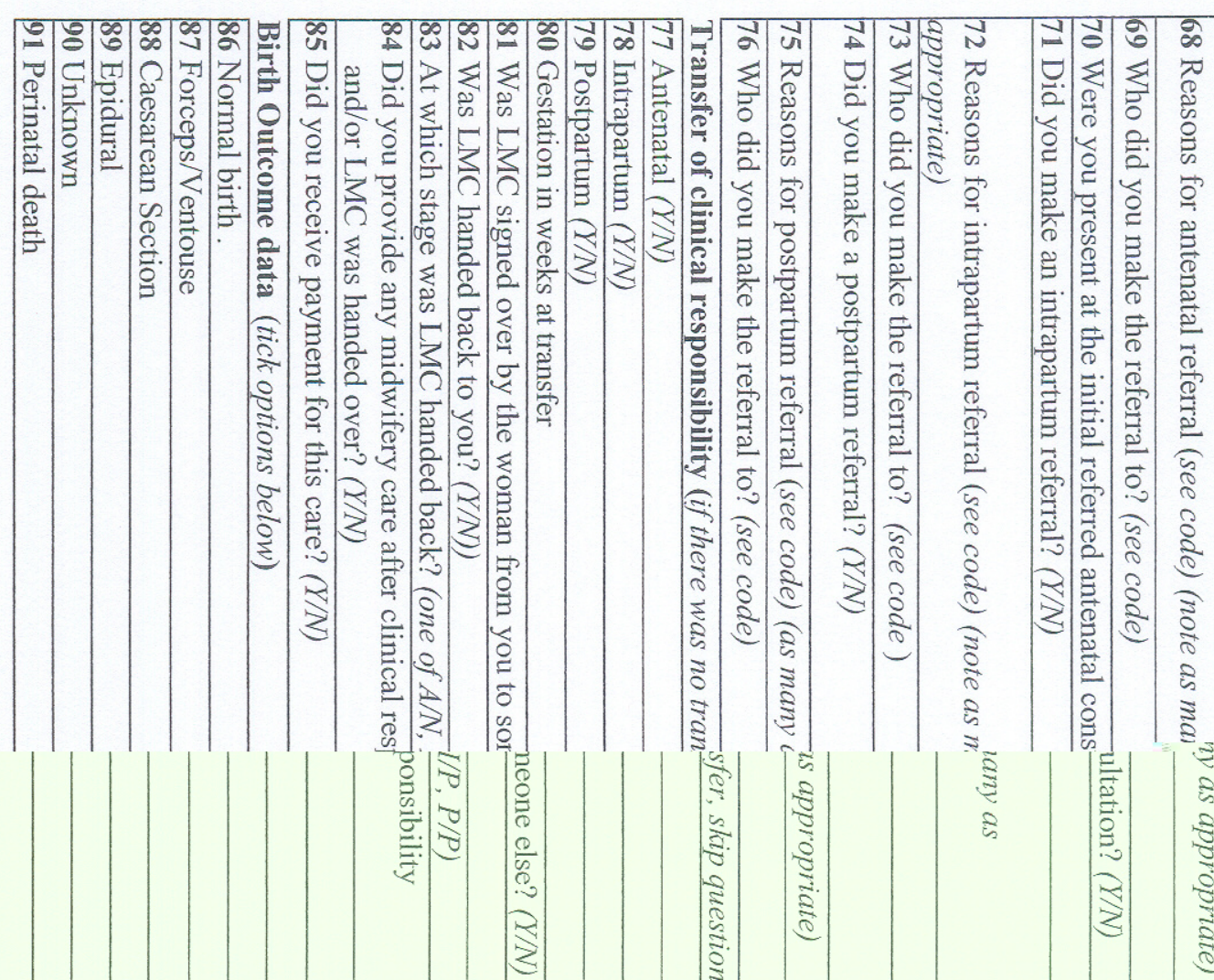

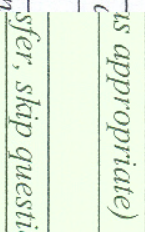

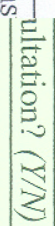

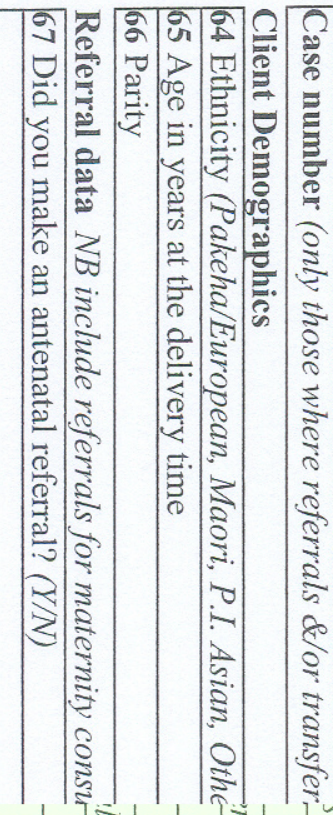
.

实
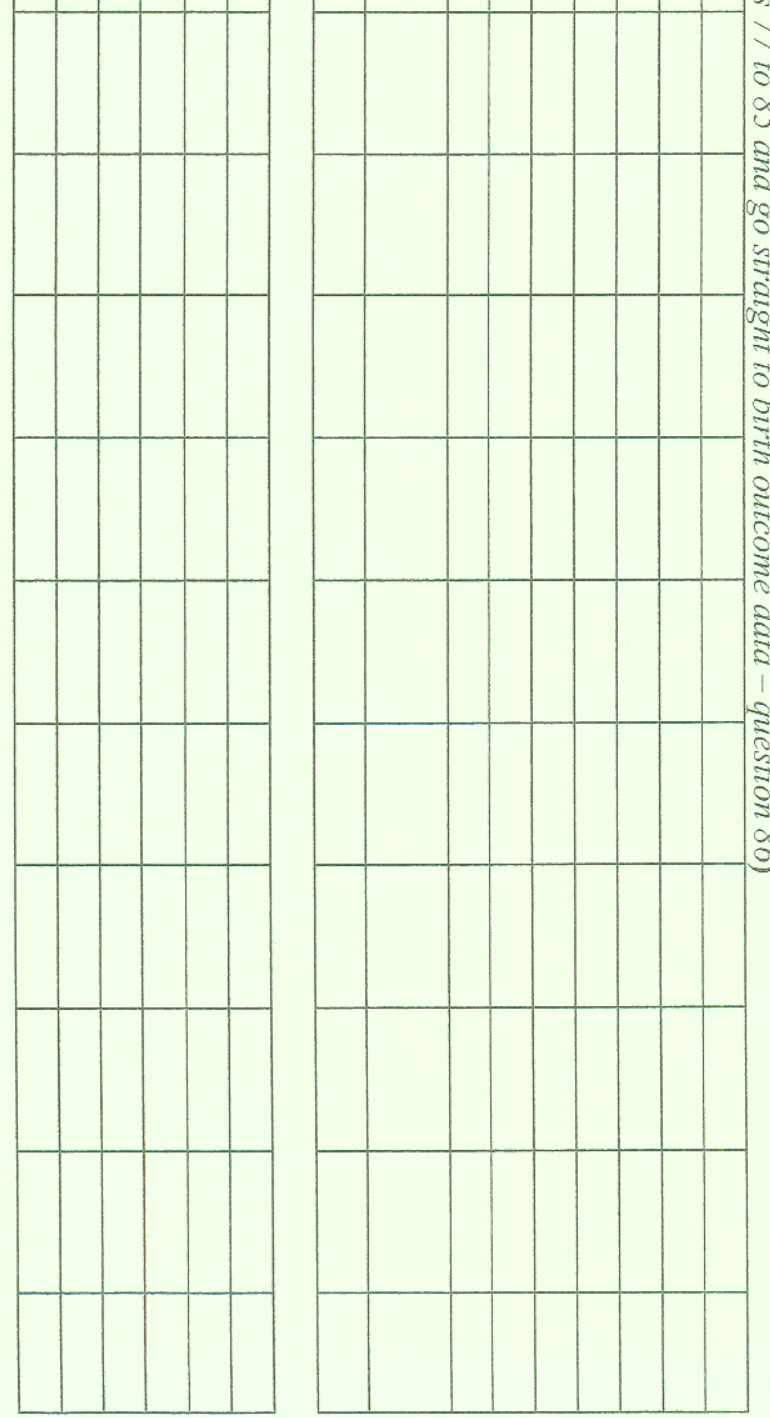

구

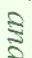
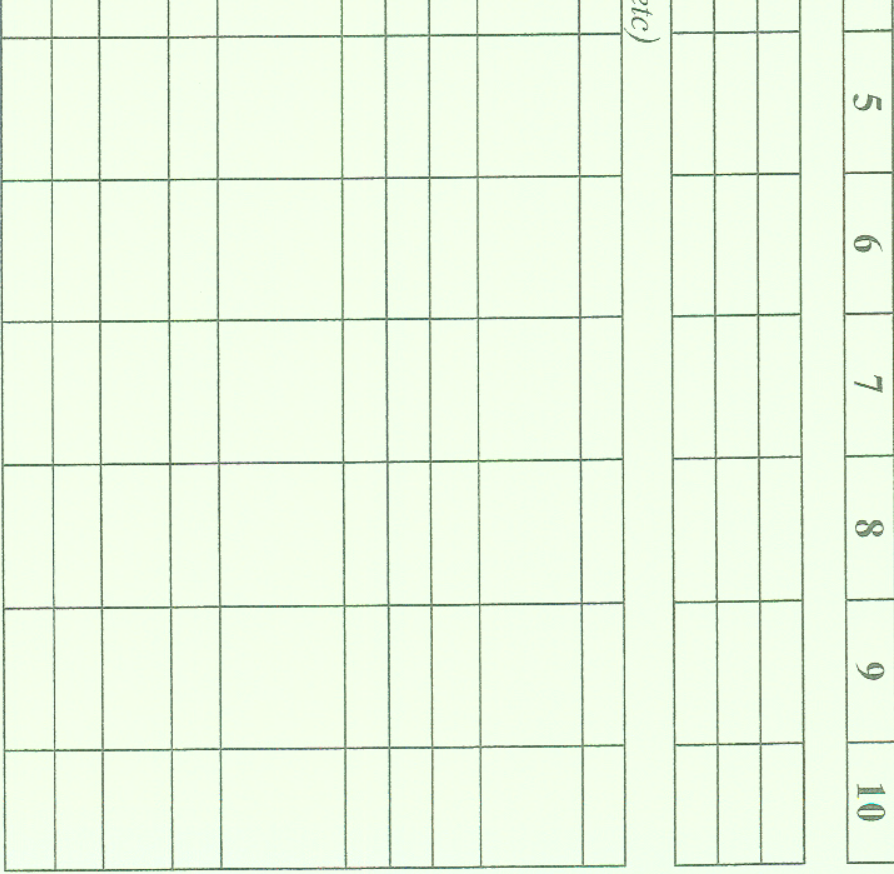
For each statement below, circle the number that is closest to your view

92 My pattern of referral is appropriate to the needs of the women I care for $\begin{array}{lllllll}1 & 2 & 3 & 4 & 5 & 6 & 7\end{array}$

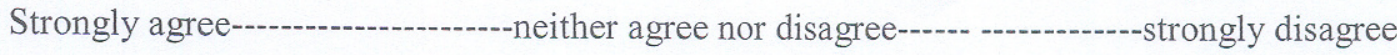

93 I believe the guidelines require me to refer more often than is necessary $\begin{array}{lllllll}1 & 2 & 3 & 4 & 5 & 6 & 7\end{array}$

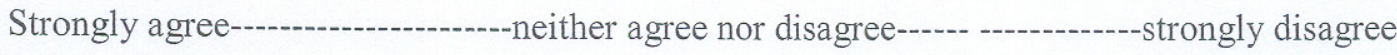

94 In my region, there is excellent collaboration between primary and secondary maternity services

$\begin{array}{lllrlrr}1 & 2 & 3 & 4 & 6 & 7\end{array}$

95 Independent midwives should care only for low risk women

$\begin{array}{lllllll}1 & 2 & 3 & 4 & 5 & 6 & 7\end{array}$

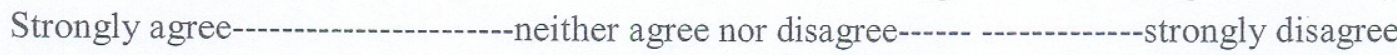

96 Risk management is an important part of my practice

$\begin{array}{llllll}1 & 2 & 3 & 4 & 6 & 7\end{array}$

97 The national referral guidelines are a useful tool when I consider the need for referral

$\begin{array}{llllllll}1 & 2 & 3 & 4 & 5 & 6 & 7\end{array}$

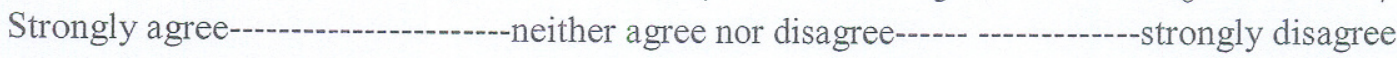

98 Obstetricians in my region in general support continuity of midwifery care $\begin{array}{lllllll}1 & 2 & 3 & 4 & 5 & 6 & 7\end{array}$

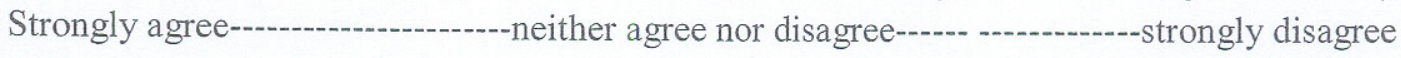

99 The medico-legal environment makes childbirth safer for women

$\begin{array}{llllll}1 & 2 & 3 & 4 & 5 & 6\end{array}$

100 The medico-legal environment restricts me in my midwifery practice $\begin{array}{llllll}1 & 2 & 3 & 4 & 5 & 6\end{array}$

Thank you for your contribution. Please send this in the freepost envelope provided to PO Box 33126 Petone 


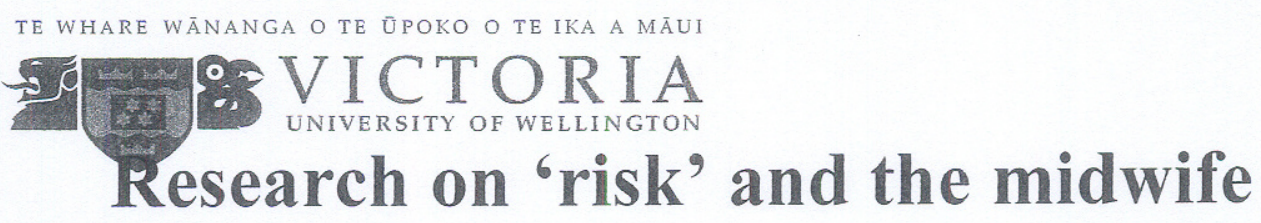

\section{Greetings fellow midwives!}

I am currently a Ph.D. student and lecturer in the Graduate School of Nursing and Midwifery at Victoria University of Wellington. My area of interest is 'risk' and the way it is expressed in maternity care in general and by midwives in New Zealand in particular. My research is looking at the interface between primary and secondary maternity care as a place where 'risk' is clearly articulated. It includes focus group discussions with midwives in a variety of New Zealand settings and a national survey of midwives' referral for consultation patterns.

My own background is one of 24 years of practice in a wide variety of roles including working in a base hospital delivery unit, in the home birth setting and in a primary health care service. I have had 5 years as the NZCOM's representative on the ACC Medical Misadventure Committee so have considerable interest the risk management practices of midwives. I have a real commitment to the role of the midwife as being with women as they give birth no matter how or where.

I realise that your time is precious but I would appreciate your taking time to complete the attached questionnaire. We are in real need in New Zealand of some factual information about how we practice and how and where we see the role of the midwife developing. Hopefully this research will assist, not only in gaining a better understanding of this but also in national policy and guideline development.

There are instructions throughout the questionnaire and coding abbreviations for the data gathering on the second page. Please do not be concerned if the numbers of consultations are few or many as I anticipate some variation in these. I acknowledge that you will need to refer to your clinical notes to fill out this survey and that some time will be needed to do so

\section{Prize draw:}

In acknowledgement of this, the return of a questionnaire will entitle you to go into a prize draw for two of the latest midwifery books

\section{"The New Midwifery", edited by Lesley Page and Joan Donley's "Birthrites: Natural vs. Unnatural Childbirth in New Zealand"}

Simply complete and tear off the section below and place it, together with the survey in the reply paid envelope attached. A research assistant will open these envelopes and immediately separate your entry in the draw from the questionnaire. There are no items 
on the survey forms which could lead to your identification. Professor Foureur (my supervisor) will do the draw once data gathering is complete. If you do not complete the questionnaire for any reason, please return it anyway.

The Wellington Regional Ethics committee and the Victoria University of Wellington Human Ethics Committee have approved this research.

Should you have any queries about this research or would like to discuss any aspect of it with me feel free either to ring me on 045864119 (hm), 044636654 (wk) or email me on joan.skinner@vuw.ac.nz. I am very happy to chat with you about it. You can also contact Professor Maralyn Foureur, my supervisor, on 044636137 with any concerns or the University Human Ethics Committee at Victoria University of Wellington PO Box 600 Wellington.

\section{Many thanks! Your participation is valued and appreciated}

Kind regards

Joan Skinner

$$
\begin{gathered}
\text { (To enter the prize draw, simply fill in details below, } \\
\text { tear along dotted line and return with questionnaire } \\
\text { in envelope provided) }
\end{gathered}
$$

NAME.

ADDRESS.

PHONE NUMBER

I AM INTERESTED IN BEING PART OF A FOCUS GROUP DISCUSSION ON

'RISK' SHOULD THERE BE ONE IN MY AREA............. YES / NO 


\section{PLEASE!! RETURN QUESTIONNAIRE IN ENVELOPE PROVIDED EVEN IF YOU HAVE NOT FILLED IT IN}

REASON ....I already have one ....I do not have a caseload

....I do not want to do it

....Other. 


\section{Focus group discussions about risk and the midwife}

Greetings. I am a Ph D candidate and midwife lecturer from the Graduate School of Nursing and Midwifery at Victoria University of Wellington. I hope to be able to conduct a focus group interview with midwives in your area. This letter is to provide you with some information about my study. I am looking at how the midwife deals with risk, both risk in the women she cares for and the medico-legal risk environment in which she works. I have conducted a national survey of referral for consultation practice and am now working on focus group discussions around the country looking what midwives understand about risk and how it impacts on their practice.

This is an important piece of work as the interface between primary and secondary maternity care is poorly researched both nationally and internationally. New Zealand midwifery is an ideal place to investigate this interface. I am very interested in looking at the views of midwives in a variety of areas.

The group discussion will take about $1 \mathrm{hr}$. It is intended that the discussion will be informal, relaxed, informative and enjoyable. I will be facilitating the discussion and the session will be taped. We will set some ground rules about the confidentiality of the discussion. The tapes will be transcribed and wiped at the completion of the study. There will be no identifiable markings on the transcripts, which will be destroyed after 5 years. Neither you nor your area will be identifiable in any research reports.

These are the areas that we are likely to be covering. You might like to have a think about some of them before deciding on coming to the group:

How does the primary/secondary interface work in your area? What are some of the difficulties? What are the risks in your work? How do you know when a situation is risky? How do think risk is expressed in practice? Do you take risks? How do you assess risk? What do you think about the referral guidelines? Do they lead to a pattern of referral appropriate to the needs of the women you care for? How do you communicate risk to the women, to the obstetrician? What do you think women feel about risk? Does risk management play an important place in your practice? What do you think is the best way of providing midwifery care for women 'at risk'?

I am really looking forward to coming to your area and hope that you will be able to join us. Please let Priscilla know id you are able to attend and feel free to ring me on either 045864119 (home) or 044636654 (work) if you have any queries. You can also contact Professor Jan Duke on 044635034 with any concerns.

Kind regards

Joan Skinner 


\section{THE MIDWIFE WITH RISK}

\section{Consent to participation in a focus group discussion}

I have been given and have understood an explanation of this research project. I have had an opportunity to ask questions and have them answered to my satisfaction. I understand that I may withdraw myself (or any information I have provided) from the project (before data collection and analysis is complete) without having to give reasons or without penalty of any sort.

I understand that any information I provide will be kept confidential to the researcher, the

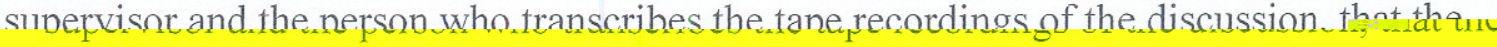
published results will not use my name, and that no opinions will be attributed to me in any way that will identify me. I understand that the tape recordings of discussions will be electronically wiped at the end of the project, and that the transcripts will be destroyed after 5 years. .

I understand that the University retains insurance cover against claims relating to harm, loss or damage suffered by participants in research projects as a result of any negligent act, error or omission by or on behalf of the University.

I understand that the data I provide will not be used for any other purpose or released to others without my written consent.

I would like to receive a summary of the results of this research when it is completed.

\section{$\mathrm{YES} / \mathrm{NO}$}

I agree to take part in this research

SIGNATURE.

NAME

DATE. 


\section{Confidentiality Contract for Transcriber}

To ensure the anonymity of the persons being interviewed

I

Of

Address.

Declare that I will not divulge any information gained from the transcripts I produce for the researcher, Joan Skinner

Signature.

Date

Researcher Joan Skinner

Signature......................... Date 